RODOLFO COSTA DE MEDEIROS

\title{
FORÇAS EM PEÇAS DE CONTRAVENTAMENTO DE TRELIÇAS DE MADEIRA
}

Dissertação apresentada à escola de Engenharia de São Carlos, da Universidade de São Paulo, para obtenção do título de Mestre em Engenharia de Estruturas.

Área de concentração: Engenharia de Estruturas

Orientador: Prof. Tit. Francisco Antonio Rocco Lahr

São Carlos 
AUTORIZO A REPRODUÇÃO E DIVULGAÇĀO TOTAL OU PARCIAL DESTE TRABALHO, POR QUALQUER MEIO CONVENCIONAL OU ELETRÔNICO, PARA FINS DE ESTUDO E PESQUISA, DESDE QUE CITADA A FONTE.

Ficha catalográfica preparada pela Seção de Tratamento da Informaçāo do Serviço de Biblioteca - EESC/USP

Medeiros, Rodolfo Costa de
M488f Forças em peças de cont

madeira / Rodolfo Costa de Medeiros ; orientador

Francisco Antonio Rocco Lahr. -- Sāo Carlos, 2010.

Dissertaçāo (Mestrado-Programa de Pós-Graduaçāo e Área de Concentraçāo em Engenharia de Estruturas) -- Escola de Engenharia de São Carlos da Universidade de São Paulo, 2010 .

1. Estruturas de madeira. 2. Contraventamento. 3. Treliças triangulares. I. Título. 


\section{FOLHA DE JULGAMENTO}

Candidato: Engenheiro RODOLFO COSTA DE MEDEIROS.

Dissertação defendida e julgada em 08/03/2010 perante a Comissão Julgadora:

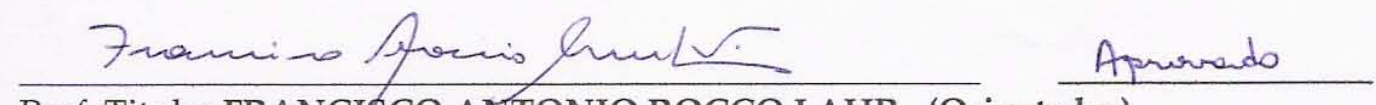

Prof. Titular FRANCISCO ANTONIO ROCCO LAHR- (Orientador)

(Escola de Engenharia de São Carlos/USP)

Prof.Dr. JOSÉ JAIRO DE SALES

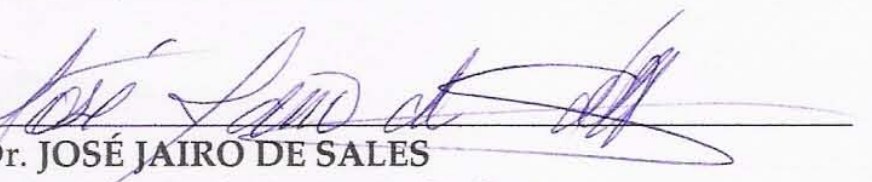

APROVA D)

(Escola de Engenharia de São Carlos/USP)

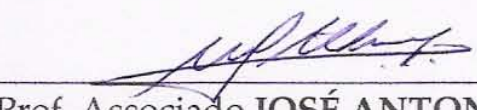

Prof. Associado JOSÉ ANTONIO MATTHIESEN

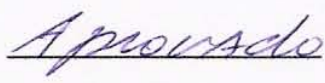

(Universidade Estadual Paulista "Julio de Mesquita Filho"/UNESP/campus de Ilha Solteira)
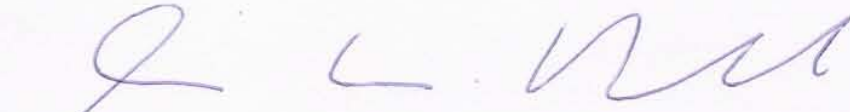

Prof. Associado MARCIO ANTONIO RAMALHO

Coordenador do Programa de Pós-Graduação em

Engenharia Civil (Engenharia de Estruturas)

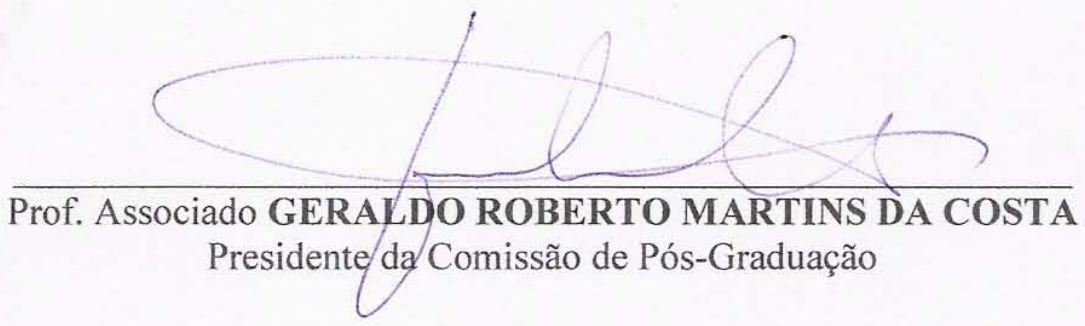





\section{RESUMO}

MEDEIROS, R. C. Forças em peças de contraventamentos de treliças de madeira. 132p. Dissertação (mestrado) - Departamento de Engenharia de Estruturas, Escola de Engenharia de São Carlos, Universidade de São Paulo, São Carlos, São Paulo, 2010.

O trabalho analisa as forças atuantes no contraventamento de treliças triangulares de coberturas de madeira por meio de modelos numéricos computacionais. Os modelos foram confeccionados no sistema de análise estrutural Strap e consideram o funcionamento do contraventamento na estrutura tridimensional. Os valores máximos das forças obtidas nos modelos tridimensionais foram comparados aos valores mínimos indicados pelo método de estimativa da NBR 7190 (1997), este, baseado na instabilidade lateral do elemento contraventado. Os valores dos modelos tridimensionais também foram comparados aos métodos do Eurocode 5, da norma sul-africana SABS 0163 e aos valores propostos por UNDERWOOD (2000), por apresentarem formulação semelhante ao da NBR 7190 (1997). Os modelos representam galpões usuais na prática, com dimensões variando de 12 a 24 metros de vão; 24 a 96 metros de comprimento; 3, 4 e 6 metros de altura do pilar. Foram analisados para os tipos de treliça Howe e Pratt, para as classes de madeira C30 e C50 e para os tipos de telhas fibrocimento, metálicas e cerâmicas, materiais mais utilizados no Brasil. Os resultados mostram que as forças atuantes no contraventamento dos modelos tridimensionais são superiores aos valores obtidos pelo método da NBR 7190 (1997) e que valores obtidos pelos métodos propostos pelo Eurocode 5 e por UNDERWOOD (2000) apresentam-se mais seguros diante do funcionamento global da estrutura.

Palavras-chave: contraventamento, treliças de madeira, estruturas de madeira 



\section{ABSTRACT}

MEDEIROS, R. C. Forces on bracing elements of timber trusses. 132p. Dissertation (master) - Departamento de Engenharia de Estruturas, Escola de Engenharia de São Carlos, Universidade de São Paulo, São Carlos, São Paulo, 2010.

This work analyzes the forces acting on the bracing bars of triangular timber trusses by computational numerical models. The models are produced in the system of structural analysis Strap and consider the operation of bracing in the three-dimensional structure. The maximum forces obtained in the three-dimensional models are compared to the minimum values indicated by the estimation method of the NBR 7190 (1997), which is based on the lateral instability of the braced element. The values of three-dimensional models are also compared to the methods of Eurocode 5, the South African standard SABS 0163 and the values proposed by Underwood (2000). The models represent sheds with usual dimensions ranging from 12 to 24 meters wide, 24 to 96 feet long, and pillar 3, 4 and 6 meters tall. Are analyzed the Pratt and Howe types of truss, the timber classes C30 and C50 and the types of tiles: cement, metal and ceramics, materials most used in Brazil. The results show that the forces acting on the bracing of the three-dimensional models are greater than those obtained by the NBR 7190 (1997) and values obtained by methods proposed by the Eurocode 5 and Underwood (2000) provides more insurance on the overall functioning structure.

Key-words: bracing, wood trusses, wood structures 



\section{SUMÁRIO}

1 INTRODUÇÃO ......................................................................................................... 17

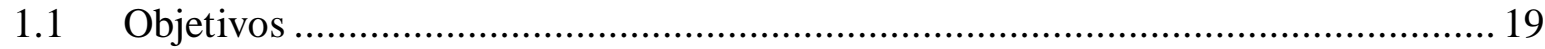

2 REVISÃO BIBLIOGRÁFICA.............................................................................. 21

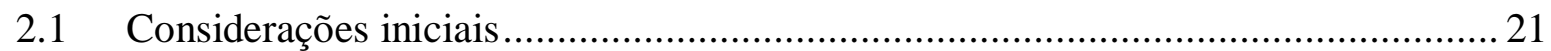

2.2 Contraventamento de estruturas de madeira ........................................................ 23

2.2.1 Contraventamento de peças comprimidas.................................................... 24

2.2.2 Estabilidade global de elementos estruturais em paralelo .............................. 28

2.2.3 Contraventamento como parte da estrutura tridimensional ............................ 34

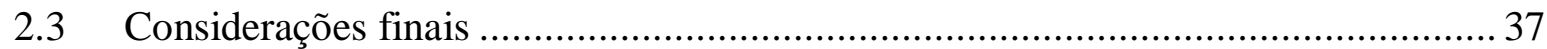

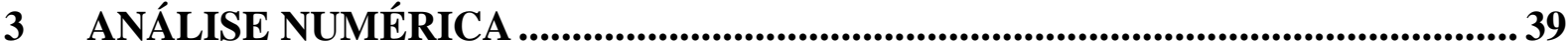

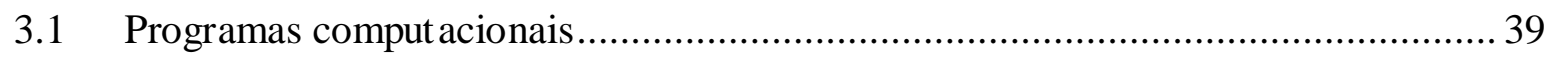

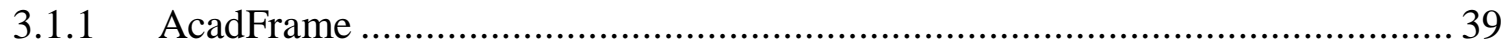

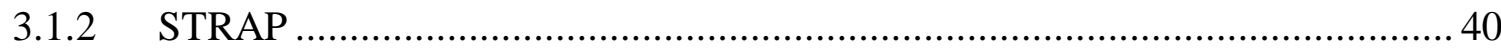

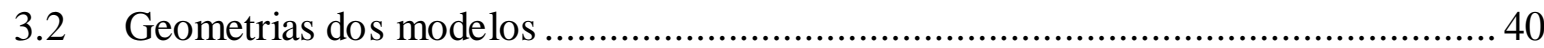

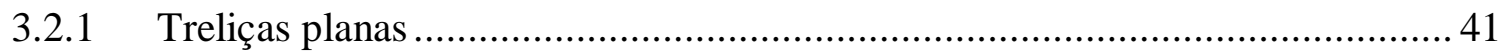

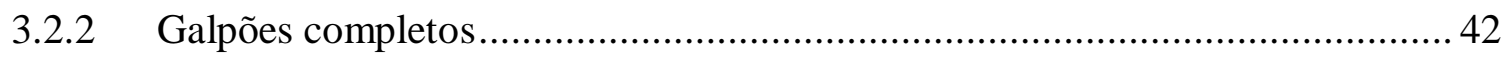

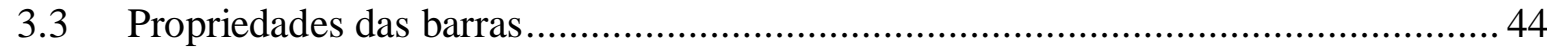

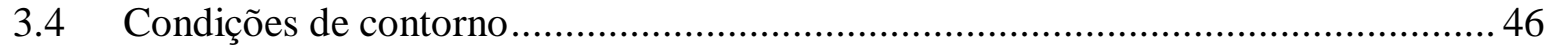

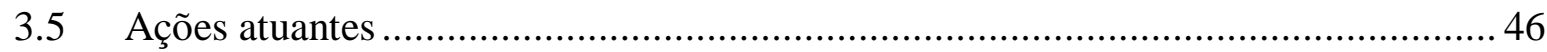

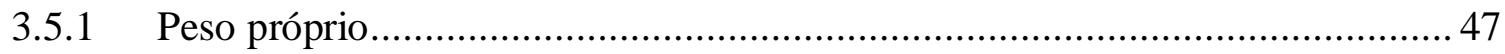

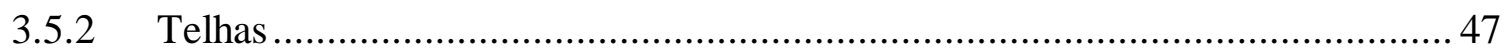

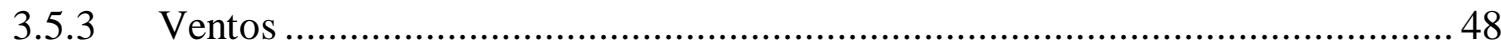

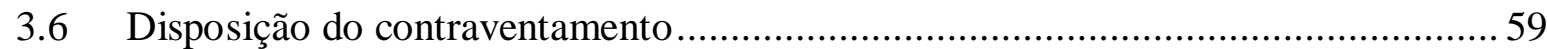

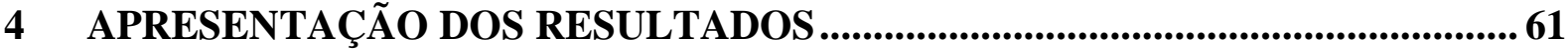

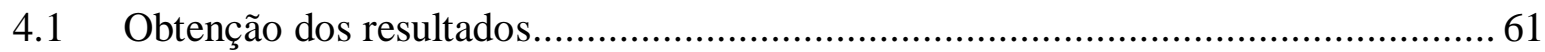




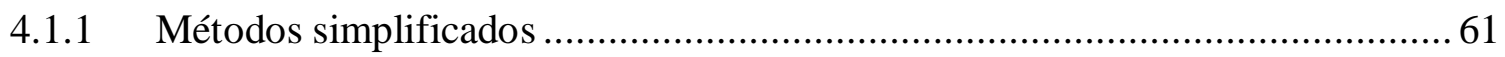

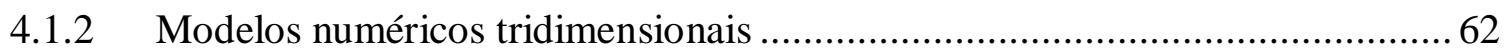

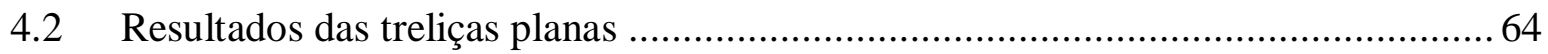

4.2.1 Treliças HOWE C50 telha fibrocimento...................................................... 65

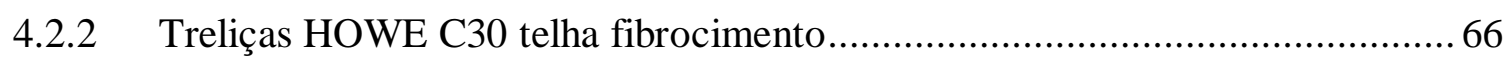

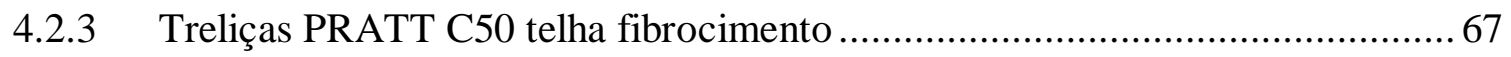

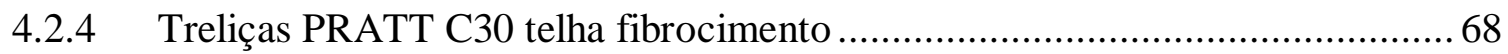

4.2.5 Treliças PRATT C50 telha metálica .............................................................. 69

4.2.6 Treliças PRATT C50 telha cerâmica ......................................................... 70

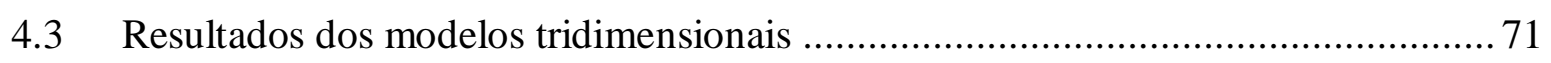

4.3.1 Modelos com contraventamento de cabos ............................................... 71

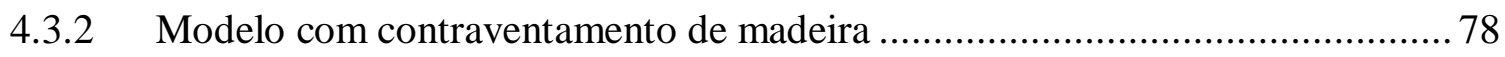

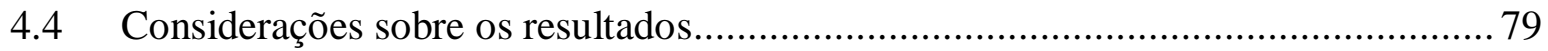

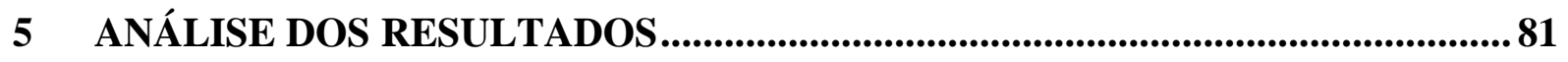

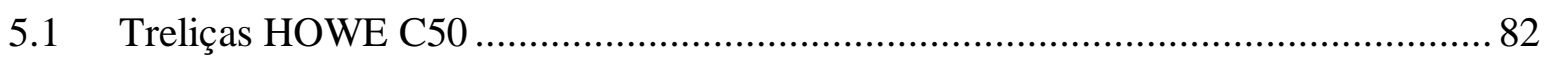

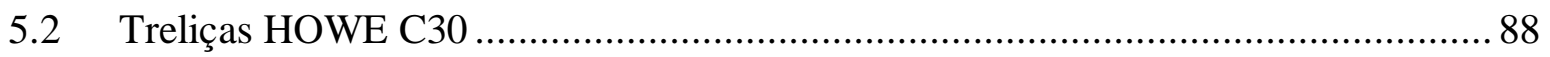

5.3 Treliças PRATT C50 contraventamento de CABOS.............................................. 94

5.4 Treliças PRATT C50 contraventamento de MADEIRA ....................................... 100

5.5 Treliças PRATT C30 ........................................................................... 106

5.6 Treliças PRATT C50 telha metálica .............................................................. 112

5.7 Treliças PRATT C50 telha cerâmica ............................................................. 118

5.8 Comparativo entre tipos de treliças e classes de resistência ................................. 122

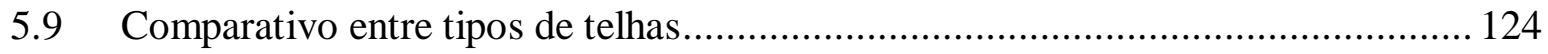

5.10 Considerações sobre a análise dos resultados ............................................ 127

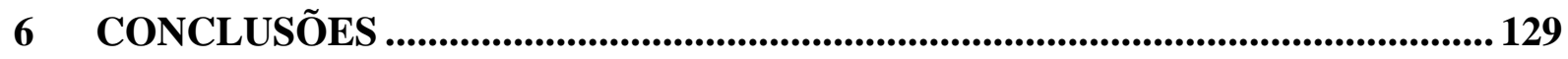

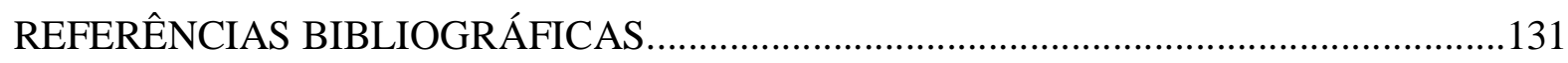




\section{LISTA DE FIGURAS}

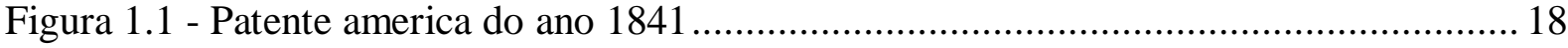

Figura 2.1 - Contraventamento de cobertura em tesouras de madeira.................................. 22

Figura 2.2 - Banco Itaú, São Paulo. (Fonte:

http://www.vitruvius.com.br/arquitextos/arq000/esp270.asp) .......................................... 22

Figura 2.3 - Deslocamento oriundo de não linearidade geométrica ..................................... 24

Figura 2.4 - Modelo de contraventamento de peça comprimida ......................................... 25

Figura 2.5 - Esforços no contraventamento de peças comprimidas da norma chilena NCh 1198 de 2006 27

Figura 2.6 - Baixa rigidez lateral das treliças (Fonte: Truss Plate Institute)......................... 29

Figura 2.7 - Possíveis casos de instabilidade de uma treliça.............................................. 29

Figura 2.8 - Flambagem lateral da tesoura completa, caso (d) da Figura 2.7 ....................... 30

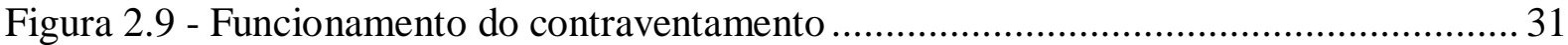

Figura 2.10 - Arranjo vertical de contraventamento ....................................................... 31

Figura 2.11 - Arranjo de esforços de contraventamento ................................................... 32

Figura 2.12 - Esquema de ação diaframa. Fonte: BARALDI (2001) ................................... 33

Figura 2.13 - Desenho esquemático do modelo estudado por ROCCO LAHR et al. (2008) .. 35

Figura 3.1 - Treliça plana tipo Howe

Figura 3.2 - Estrutura completa de galpão com treliça tipo Howe e contraventamento de barras de aço

Figura 3.3 - Desenho esquemático das dimensões de um galpão 43

Figura 3.4 - Carregamento do peso próprio (em daN e m) da tesoura tipo C50 (970 daN/m³) e das terças representadas pelas cargas concentradas 47

Figura 3.5 - Carregamento de telhas fibrocimento 48

Figura 3.6 - Desenho esquemático de distâncias e faces de aplicação da ação estática dos ventos 
Figura 3.7 - Desenho esquemático de distâncias e faces de aplicação da ação estática dos ventos 53

Figura 3.8 - Carregamento de sobrepressão do vento na direção longitudinal (vento a $0^{\circ}$ )..... 54

Figura 3.9 - Sentidos dos sinais dos coeficientes de pressão 56

Figura 4.1 - Deslocamentos e deformada da Treliça 01 Pratt C50 (STRAP) 62

Figura 4.2 - Deslocamentos e deformada do Galpão 01 Pratt C50. 63

Figura 4.3 - Forças axiais máximas do Galpão 01. 64

Figura 5.1 - Exemplo explicativos das tabelas 81 


\section{LISTA DE TABELAS}

Tabela 2.1 - Representação da Tabela 17 da NBR 7190/1997. 26

Tabela 2.2 - Comparação de esforços obtidos, nos modelos tri e bidimensionais, por ROCCO

LAHR et al. (2008). 36

Tabela 2.3 - Esforços obtidos nas demais peças do modelo tridimensional 36

Tabela 3.1 - Geometrias dos modelos de treliças 41

Tabela 3.2 - Dimensões dos galpões estudados (unidades em metros) 43

Tabela 3.3 - Classes de resistência das coníferas. (Fonte: CALIL JR., 2008) 44

Tabela 3.4 - Classes de resistência das dicotiledôneas. (Fonte: CALIL JR., 2008) 44

Tabela 3.5 - Dimensões das seções das barras da treliça . 45

Tabela 3.6 - Valores de S2 para os modelos considerados 50

Tabela 3.7 - Valores de Vk e ação estática q 50

Tabela 3.8 - Ação estática do vento multiplicada pelos coeficientes de pressão 51

Tabela 3.9 - Distâncias e coeficientes de pressão externa 52

Tabela 3.10 - Ação estática dos ventos em cada face 53

Tabela 3.11 - Ação interna dos ventos 55

Tabela 3.12 - Formulação para obtenção dos coeficientes de pressão para coberturas isoladas 56

Tabela 3.13 - Coeficientes de pressão para coberturas isoladas 57

Tabela 3.14 - Ação dos ventos em coberturas isoladas. 57

Tabela 4.1 - Forças nos contraventamentos segundo os métodos simplificados (HOWE C50)

Tabela 4.2 - Forças nos contraventamentos segundo os métodos simplificados (HOWE C30) 66

Tabela 4.3 - Forças nos contraventamentos segundo os métodos simplificados (PRATT C50) 
Tabela 4.4 - Forças nos contraventamentos segundo os métodos simplificados (PRATT C30)

Tabela 4.5 - Forças nos contraventamentos segundo os métodos simplificados (PRATT C50

telha metálica).

Tabela 4.6 - Forças nos contraventamentos segundo os métodos simplificados (PRATT C50

cerâmica) 70

Tabela 4.7 - Forças axiais máximas no banzo e no contraventamento (HOWE C50)............. 72

Tabela 4.8 - Forças axiais máximas no banzo e no contraventamento (HOWE C30)............. 73

Tabela 4.9 - Forças axiais máximas no banzo e no contraventamento (PRATT C50) ............. 74

Tabela 4.10 - Forças axiais máximas no banzo e no contraventamento (PRATT C30) .......... 75

Tabela 4.11 - Forças axiais máximas no banzo e no contraventamento (PRATT C50 metálica)

Tabela 4.12 - Forças axiais máximas no banzo e no contraventamento (PRATT C50 cerâmica)

Tabela 4.13 - Forças axiais máximas no banzo e no contraventamento (PRATT C50)........... 78

Tabela 5.1 - Comparativo entre o método da NBR 7190 (1997) e os modelos tridimensionais

(HOWE C50).

Tabela 5.2 - Comparativo entre o método do EUROCODE 5 e os modelos tridimensionais

(HOWE C50)

Tabela 5.3 - Comparativo entre o método de UNDERWOOD (2000) e os modelos

tridimensionais (HOWE C50)

Tabela 5.4 - Comparativo entre o método do SABS 0163 e os modelos tridimensionais

(HOWE C50) .85

Tabela 5.5 - Comparativo entre o método da NBR 7190 (1997) e os modelos tridimensionais

(HOWE C30) 88 
Tabela 5.6 - Comparativo entre o método do EUROCODE 5 e os modelos tridimensionais

(HOWE C30)

Tabela 5.7 - Comparativo entre o método de UNDERWOOD (2000) e os modelos

tridimensionais (HOWE C30) 90

Tabela 5.8 - Comparativo entre o método do SABS 0163 e os modelos tridimensionais

(HOWE C30)

Tabela 5.9 - Comparativo entre o método da NBR 7190 (1997) e os modelos tridimensionais

(PRATT C50)

Tabela 5.10 - Comparativo entre o método do EUROCODE 5 e os modelos tridimensionais (PRATT C50)

Tabela 5.11 - Comparativo entre o método de UNDERWOOD (2000) e os modelos tridimensionais (PRATT C50) 96

Tabela 5.12 - Comparativo entre o método do SABS 0163 e os modelos tridimensionais (PRATT C50)

Tabela 5.13 - Comparativo entre o método da NBR 7190 (1997) e os modelos tridimensionais (PRATT C50 madeira). 100

Tabela 5.14 - Comparativo entre o método do EUROCODE 5 e os modelos tridimensionais (PRATT C50 madeira) 101

Tabela 5.15 - Comparativo entre o método de UNDERWOOD (2000) e os modelos

tridimensionais (PRATT C50 madeira) 102

Tabela 5.16 - Comparativo entre o método do SABS 0163 e os modelos tridimensionais (PRATT C50 madeira) 103

Tabela 5.17 - Comparativo entre o método da NBR 7190 (1997) e os modelos tridimensionais (PRATT C30) 106

Tabela 5.18 - Comparativo entre o método do EUROCODE 5 e os modelos tridimensionais (PRATT C30) 
Tabela 5.19 - Comparativo entre o método de UNDERWOOD (2000) e os modelos

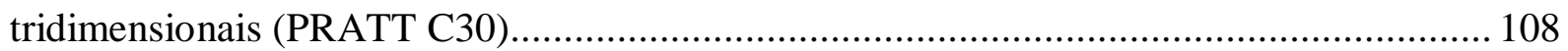

Tabela 5.20 - Comparativo entre o método do SABS 0163 e os modelos tridimensionais

(PRATT C30) 109

Tabela 5.21 - Comparativo entre o método da NBR 7190 (1997) e os modelos tridimensionais (PRATT C50 telha metálica).

Tabela 5.22 - Comparativo entre o método do EUROCODE 5 e os modelos tridimensionais

(PRATT C50 telha metálica)

Tabela 5.23 - Comparativo entre o método de UNDERWOOD (2000) e os modelos

tridimensionais (PRATT C50 telha metálica)

Tabela 5.24 - Comparativo entre o método do SABS 0163 e os modelos tridimensionais

(PRATT C50 telha metálica) 115

Tabela 5.25 - Comparativo entre o método da NBR 7190 (1997) e os modelos tridimensionais (PRATT C50 telha cerâmica) 118

Tabela 5.26 - Comparativo entre o método do EUROCODE 5 e os modelos tridimensionais

(cerâmica) 118

Tabela 5.27 - Comparativo entre o método de UNDERWOOD (2000) e os modelos

tridimensionais (PRATT C50 telha cerâmica)

Tabela 5.28 - Comparativo entre o método do SABS 0163 e os modelos tridimensionais

(PRATT C50 telha cerâmica)

Tabela 5.29 - Forças de compressão máximas nos banzos das treliças de telha fibrocimento

Tabela 5.30 - Forças de compressão máximas no contraventamento 123

Tabela 5.31 - Forças de compressão máximas nos banzos das treliças Pratt C50 124

Tabela 5.32 - Forças de compressão máximas no contraventamento das treliças Pratt C50.125

Tabela 5.33 - Forças de tração máximas no contraventamento das treliças Pratt C50. 126 


\section{LISTA DE GRÁFICOS}

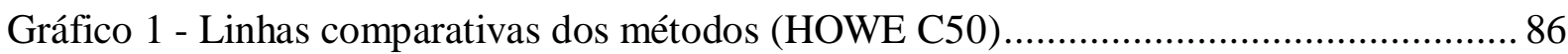

Gráfico 2 - Colunas comparativas do método NBR 7190 (HOWE C50) .............................. 86

Gráfico 3 - Colunas comparativas do método EUROCODE 5 (HOWE C50)....................... 86

Gráfico 4 - Colunas comparativas do método UNDERWOOD (HOWE C50)...................... 87

Gráfico 5 - Colunas comparativas do método SABS 0163 (HOWE C50) …..........................87

Gráfico 6 - Linhas comparativas dos métodos (HOWE C30)............................................. 92

Gráfico 7 - Colunas comparativas do método NBR 7190 (HOWE C30) .............................. 92

Gráfico 8 - Colunas comparativas do método EUROCODE 5 (HOWE C30)....................... 92

Gráfico 9 - Colunas comparativas do método UNDERWOOD (HOWE C30)...................... 93

Gráfico 10 - Colunas comparativas do método SABS 0163 (HOWE C30)........................... 93

Gráfico 11 - Linhas comparativas dos métodos (PRATT C50 cabos)..................................98

Gráfico 12 - Colunas comparativas do método NBR 7190 (PRATT C50 cabos)................... 98

Gráfico 13 - Colunas comparativas do método EUROCODE 5 (PRATT C50 cabos)............ 98

Gráfico 14 - Colunas comparativas do método UNDERWOOD (PRATT C50 cabos) .......... 99

Gráfico 15 - Colunas comparativas do método SABS 0163 (PRATT C50 cabos) ................. 99

Gráfico 16 - Linhas comparativas dos métodos (PRATT C50 madeira).............................. 104

Gráfico 17 - Colunas comparativas do método NBR 7190 (PRATT C50 madeira) .............. 104

Gráfico 18 - Colunas comparativas do método EUROCODE 5 (PRATT C50 madeira)...... 104

Gráfico 19 - Colunas comparativas do método UNDERWOOD (PRATT C50 madeira)..... 105

Gráfico 20 - Colunas comparativas do método SABS 0163 (PRATT C50 madeira)............ 105

Gráfico 21 - Linhas comparativas dos métodos (PRATT C30) ....................................... 110

Gráfico 22 - Colunas comparativas do método NBR 7190 (PRATT C30)........................... 110

Gráfico 23 - Colunas comparativas do método EUROCODE 5 (PRATT C30) ................... 110

Gráfico 24 - Colunas comparativas do método UNDERWOOD (PRATT C30) .................. 111

Gráfico 25 - Colunas comparativas do método SABS 0163 (PRATT C30)......................... 111 
Gráfico 26 - Linhas comparativas dos métodos (PRATT C50 telha metálica). 116

Gráfico 27 - Colunas comparativas do método NBR 7190 (PRATT C50 telha metálica) .... 116

Gráfico 28 - Colunas comparativas do método EUROCODE 5 (PRATT C50 telha metálica) 116

Gráfico 29 - Colunas comparativas do método UNDERWOOD (PRATT C50 telha metálica)

Gráfico 30 - Colunas comparativas do método SABS 0163 (PRATT C50 telha metálica)... 117

Gráfico 31 - Linhas comparativas dos métodos (PRATT C50 telha cerâmica) ..................... 120

Gráfico 32 - Colunas comparativas do método NBR 7190 (PRATT C50 telha cerâmica) ... 120

Gráfico 33 - Colunas comparativas do método EUROCODE 5 (PRATT C50 telha cerâmica)

Gráfico 34 - Colunas comparativas do método UNDERWOOD (PRATT C50 telha cerâmica)

Gráfico 35 - Colunas comparativas do método SABS 0163 (PRATT C50 telha cerâmica).. 121

Gráfico 36 - Colunas comparativas das forças $(\mathrm{kN})$ de compressão máximas nos banzos ... 122

Gráfico 37 - Colunas comparativas das forças $(\mathrm{kN})$ de compressão máximas no

contraventamento

Gráfico 38 - Colunas das forças máximas de compressão nos banzos 124

Gráfico 39 - Colunas das forças máximas de compressão no contraventamento 125

Gráfico 40 - Colunas das forças máximas de tração no contraventamento 126 


\section{INTRODUÇÃO}

O contraventamento é um subsistema estrutural de travamento das estruturas planas com a finalidade de manter a estabilidade dos elementos que a compõem. Este subsistema garante que a estrutura trabalhe de acordo com os critérios de estabilidade considerados em projeto, para cada elemento constituinte da estrutura.

A forma mais usual de estrutura plana de edificações é a treliça, também conhecida como tesoura. As treliças são largamente utilizadas em estruturas de coberturas de edificações e o contraventamento é parte indispensável deste tipo de sistema estrutural, pois um modelo ideal simplificado de análise estrutural considera apenas os deslocamentos no plano da treliça. Porém as estruturas apresentam imperfeições geométricas e heterogeneidade das propriedades físicas dos materiais que podem provocar deslocamentos fora do plano da estrutura que devem ser previstos em projeto e combatidos pelo contraventamento.

Há registros da preocupação com o contraventamento de treliças de madeira do ano de 1841, uma patente (Price \& Phillins Truss Bridge) registrada pelo UNITED STATES PATENT OFFICE no estado de Maryland, Estados Unidos, que descreve o contraventamento de treliças planas de pontes e estruturas similares (MANNER OF FASTENING AND COMBINING THE TRUSS-FRAMES OF BRIDGES, \&c.). Mostrada na Figura 1.1.

O atual modelo de cálculo de esforços em contraventamento, apresentado pela NBR 7190 (1997), considera a instabilidade lateral dos elementos empenados com curvatura da ordem de L/300. O modelo estrutural de treliças planas apresenta-se simplista perante a tecnologia e ferramentas computacionais hoje disponíveis. Apesar de o modelo plano apresentar resultados satisfatórios, omite a influência do contraventamento na estrutura tridimensional e os esforços atuantes nos elementos do contraventamento. Modelos tridimensionais que representam a estrutura completa apresentam esforços nos elementos de contraventamento superiores ao do modelo estabelecido pela NBR 7190 (1997) de estimativa 
de esforços mínimos - na ausência de uma análise rigorosa - no contraventamento, podendo, assim, resultar em valores contra a segurança das estruturas.

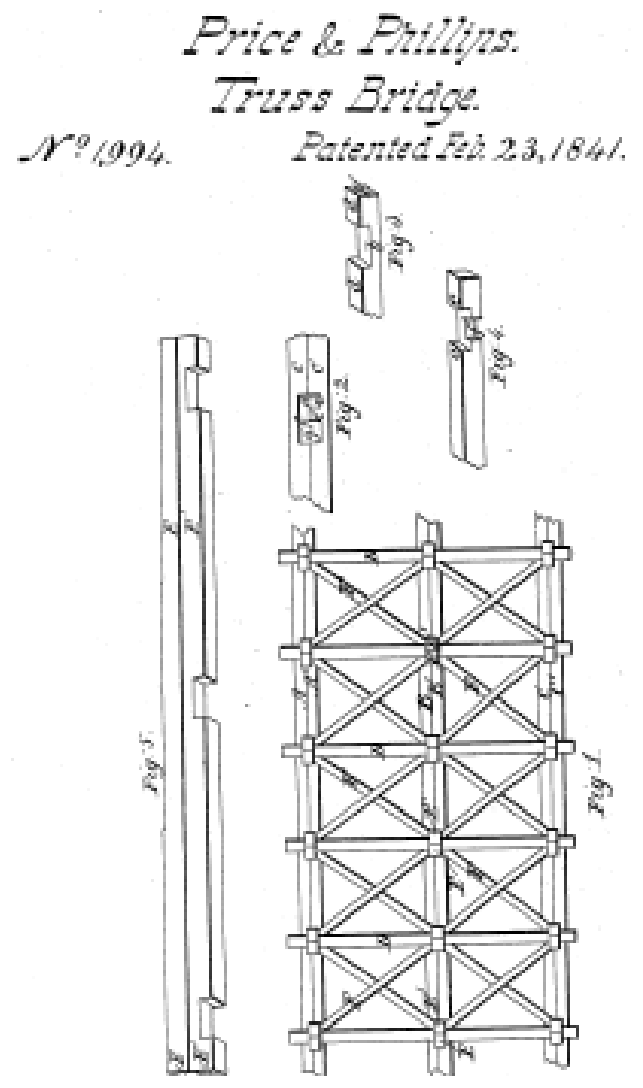

Figura 1.1 - Patente america do ano 1841

No Brasil, apesar da maioria das coberturas serem de estruturas de madeira, não há uma cultura forte - como em países da Europa e América do Norte - em utilização de estruturas de madeira. Fato este que leva muitas vezes à ausência do projeto estrutural, e a estrutura fica a cargo do carpinteiro. Assim, observam-se inúmeras patologias e acidentes em coberturas de madeira devido à ausência de um sistema de contraventamento adequado.

O uso da madeira como material estrutural apresenta-se bem no quadro mundial atual, tendo em vista a procura por materiais renováveis e que não agridam o meio ambiente. A madeira é um material renovável, quando seu uso se dá de maneira planejada, “junto de uma atividade florestal madeireira sustentável” como demonstram RAMPAZZO e SPONCHIADO (2000). 
Este trabalho busca analisar as forças atuantes no contraventamento de treliças de madeira. Na análise é feita a modelagem numérica dos galpões por meio da ferramenta computacional STRAP. Os modelos apresentados buscam retratar estruturas de média a grandes proporções e sem a consideração dos elementos de vedação (painel diafragma) resistindo a esforços, assim apresentando valores dos casos mais desfavoráveis, para avaliação de valores mínimos na ausência de uma análise rigorosa.

\subsection{Objetivos}

Os objetivos deste trabalho são:

- estimar as forças de projeto atuantes nos elementos de contraventamento de estruturas de treliças triangulares de madeira por meio de modelos numéricos computacionais;

- comparar as forças obtidas na modelagem tridimensional com os valores obtidos pelo método de estimativa apresentado na NBR 7190 (1997) e outras normas internacionais. 


\section{REVISÃO BIBLIOGRÁFICA}

Este capítulo apresenta uma visão geral de estruturas de contraventamento, suas aplicações e atuais métodos de estimativa de esforços e cálculo.

\subsection{Considerações iniciais}

O objetivo do contraventamento é travar os nós da estrutura conforme dispostos no modelo de cálculo. O nome relaciona o vento pelo fato do mesmo ser o principal responsável por deslocamentos horizontais fora do plano de disposição da estrutura. O contraventamento, por sua vez, tem como função inibir esses deslocamentos que possam causar efeitos de segunda ordem na estrutura.

O contraventamento, da forma aqui estudada, é utilizado em estruturas de ligações pouco rígidas que venham a permitir seus movimentos. Como exemplo, a Figura 2.1 mostra o contraventamento constituinte do vão de extremidade da cobertura em tesouras de madeira do SESC São Carlos, e a Figura 2.2 mostra o contraventamento aparente de um edifício de estrutura metálica como elemento de fachada. 


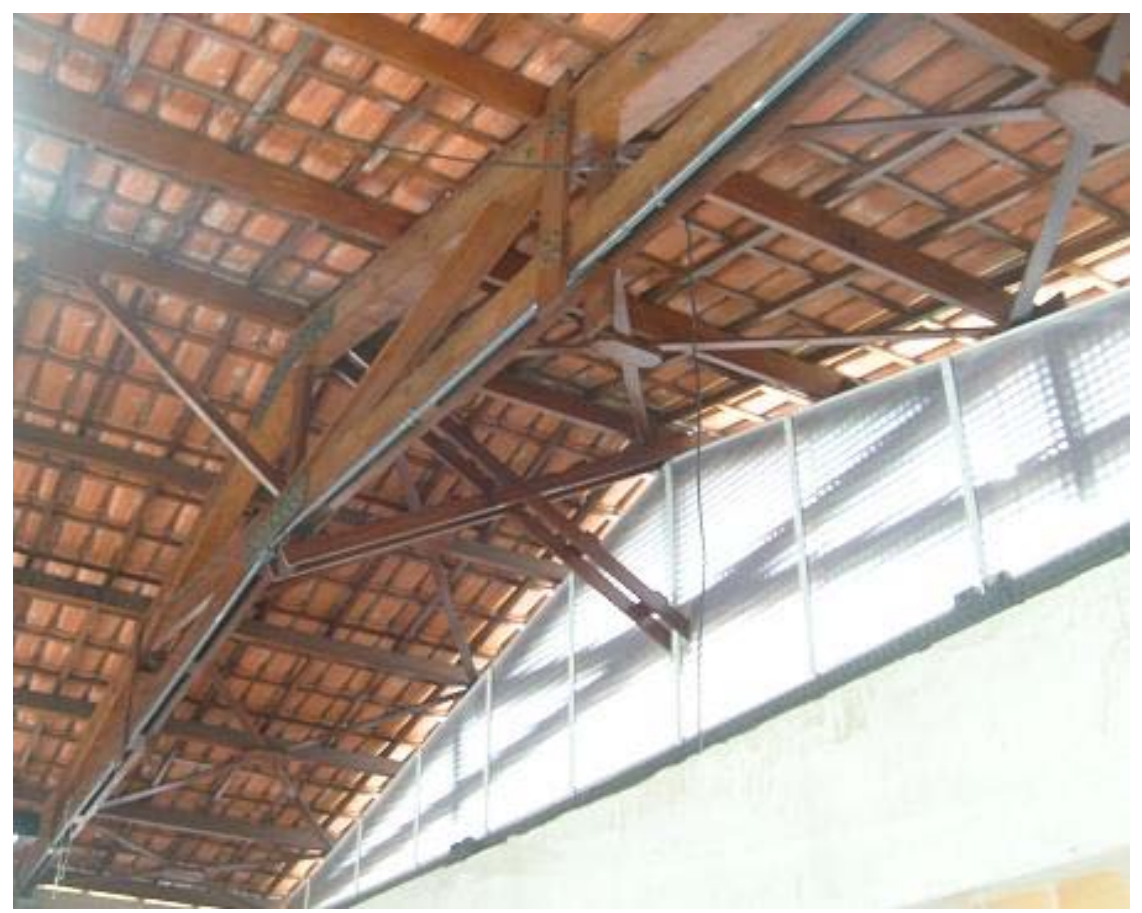

Figura 2.1 - Contraventamento de cobertura em tesouras de madeira

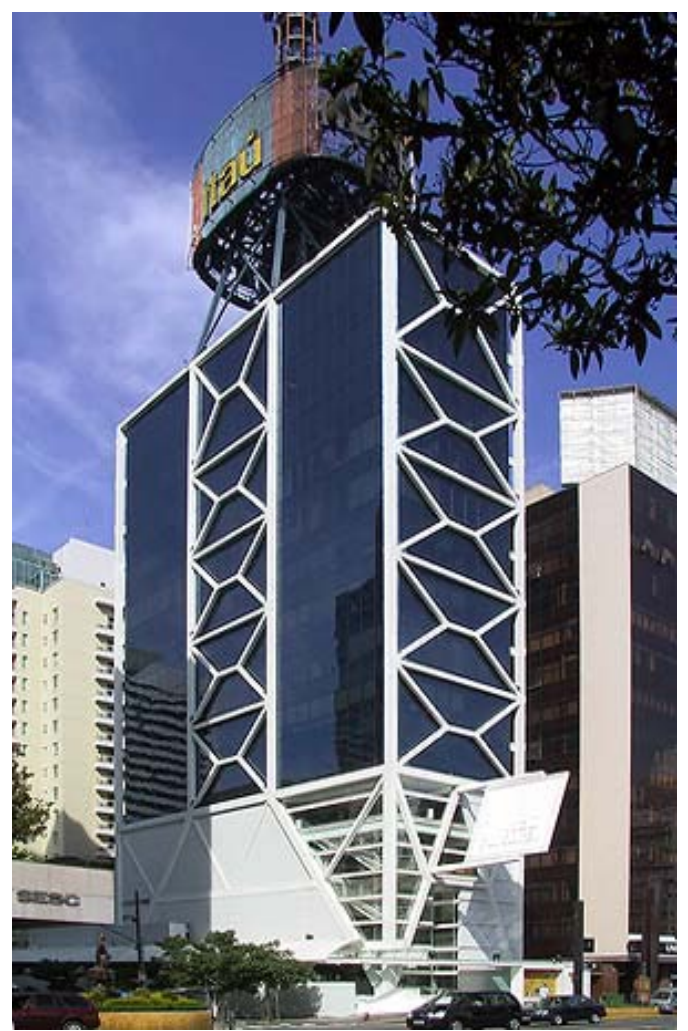

Figura 2.2 - Banco Itaú, São Paulo. (Fonte: http://www.vitruvius.com.br/arquitextos/arq000/esp270.asp) 
Um sistema de contraventamento inadequado foi o responsável de vários acidentes da engenharia, assim, deve ser integrante essencial das estruturas planas. A Figura 2.8 apresenta um caso de perda de estabilidade da cobertura de uma área de lazer, que por sua vez veio a ser interditada e recuperada a tempo, evitando a ruína da estrutura.

\subsection{Contraventamento de estruturas de madeira}

Nas estruturas de madeira, os sistemas de contraventamento são: o travamento de peças isoladas; de elementos estruturais principais; e de estruturas como um todo.

Por simplificação dos modelos numéricos de cálculo, em muitos casos são considerados deslocamentos apenas em uma direção do elemento estrutural, no seu plano de maior rigidez. Porém, a não linearidade e esforços fora do plano principal de atuação da estrutura provocam deslocamentos transversais, a este plano principal, que devem ser previstos em projeto.

A não linearidade é subdividida em dois casos: não linearidade geométrica, que são as imperfeições geométricas das peças ou da estrutura, como um empenamento da peça, oriunda de secagem ou formação natural do tronco de uma árvore, ou a imperfeição do prumo da estrutura; e não linearidade física, causada pela não homogeneidade das propriedades físicas do material estrutural, sendo esta inevitável em estruturas de madeira por ser um material fabricado pela natureza, anisotrópico por sua diferente disposição das estruturas celulares ao longo da árvore.

Exemplos de contraventamento em estruturas de madeira são: vigas de um assoalho travadas por peças dispostas transversalmente ou pelo próprio piso; pilares ou escoramentos comprimidos. Sua função pode ser: a diminuição do comprimento de flambagem de peças comprimidas isoladas ou constituintes de treliças; e até a quebra do comprimento de flambagem de peças tracionadas esbeltas que possam apresentar vibrações excessivas.

A Figura 2.3 apresenta uma deformação de segunda ordem provocada pela aplicação de uma força axial em uma peça com uma imperfeição geométrica inicial. 

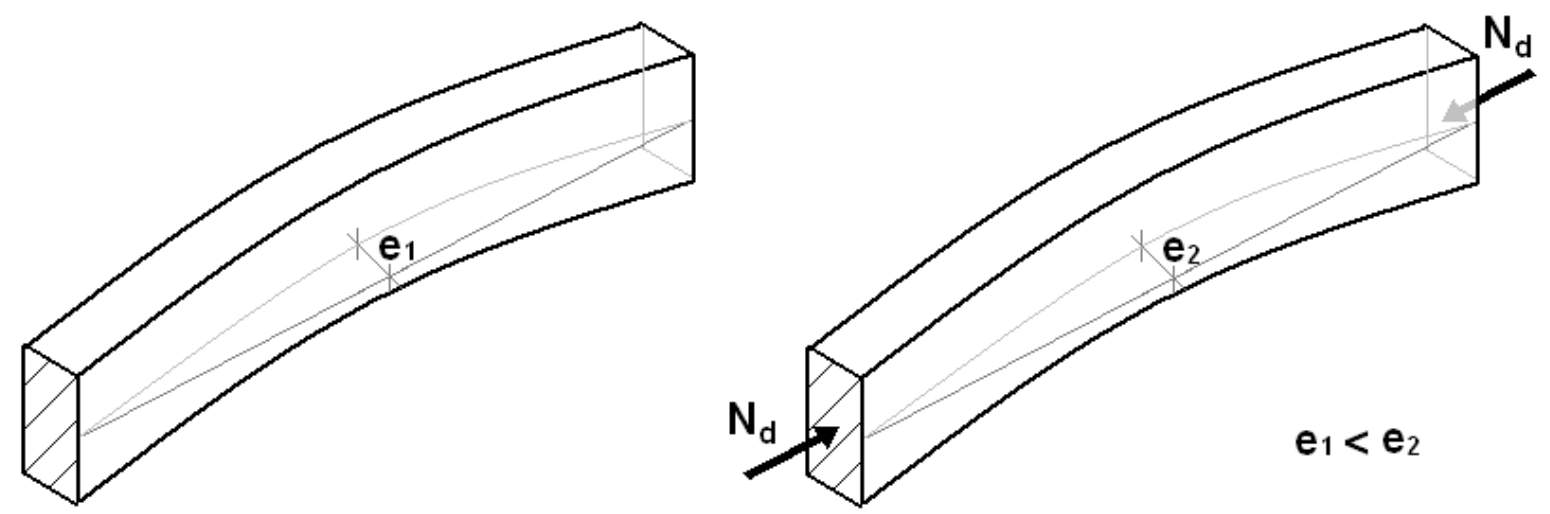

Figura 2.3 - Deslocamento oriundo de não linearidade geométrica

O documento normativo NBR 7190 de 1997, que rege os procedimentos de projeto e execução de estruturas de madeira, separa em dois grupos os elementos contraventados para determinação da rigidez e do esforço de dimensionamento dos mesmos: as peças comprimidas e os elementos estruturais em paralelo.

Autores de trabalhos que tratam do assunto - como BARALDI (2001) e ROCCO LAHR et al. (2008) - apontam métodos de análise estrutural mais rigorosos que consideram a ação resistente dos elementos de vedação ou o funcionamento global da estrutura.

Algumas normas internacionais apresentam formulações similares à apresentada pela NBR 7190 (1997), baseadas na instabilidade lateral do elemento isolado. Porém, a análise tridimensional da estrutura resulta em valores mais altos.

\subsubsection{Contraventamento de peças comprimidas}

A seguir são expostos os métodos de cálculo pesquisados que serviram como base da análise dos resultados obtidos pelos modelos estudados neste trabalho.

\subsubsection{NBR 7190 (1997)}

Para as peças comprimidas, a norma permite que em cada nó contraventado seja considerada uma força $F_{1 d}$ de valor mínimo $N_{d} / 150$, sendo $N_{d}$ a força de compressão de 
cálculo da peça e o $\mathrm{N}_{d} / 150$ oriundo da sua curvatura inicial com flechas da ordem de $\mathrm{L} / 300$ do comprimento do arco correspondente.

A Figura 2.4 mostra a representação do modelo numérico para análise de uma peça estrutural bi-rotulada e contraventada por apoios elásticos de rigidez $\mathrm{K}_{\mathrm{br}}$ submetida a uma ação axial $\mathrm{N}_{\mathrm{d}}$.

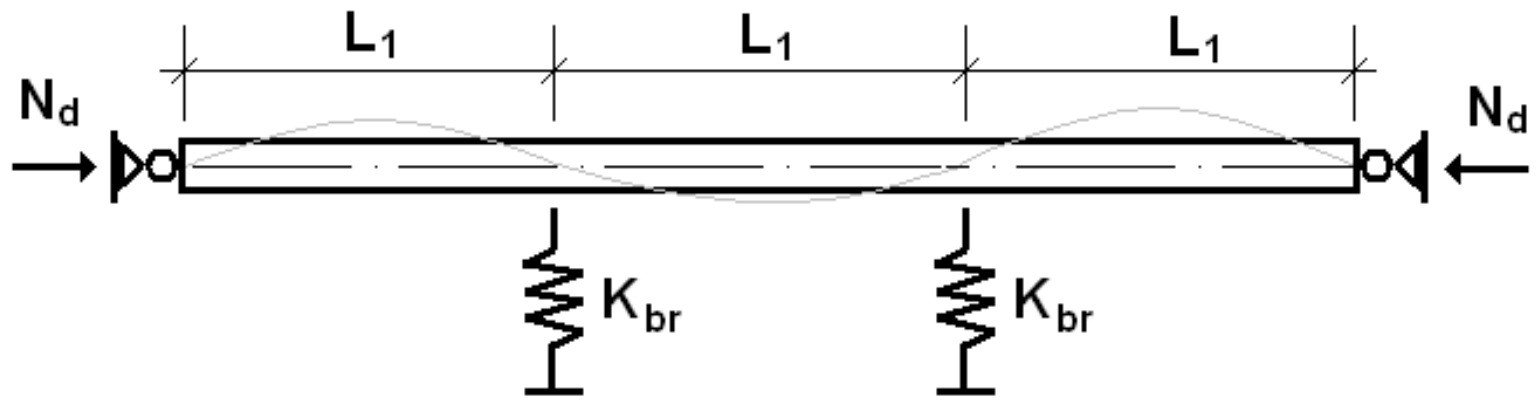

Figura 2.4 - Modelo de contraventamento de peça comprimida

A estrutura de travamento deve ter uma rigidez mínima $\mathrm{K}_{\mathrm{br}, 1}$ dada pela Expressão 2.1:

$$
K_{b r, 1, \min }=2 \cdot \alpha_{m} \frac{\left(\pi^{2} \cdot E_{c 0, e f} \cdot I_{2}\right)}{L_{1}^{3}}
$$

Onde:

$$
\begin{aligned}
& \alpha_{m}=1+\cos \frac{\pi}{m} \\
& m=\text { número de segmentos contraventados } \\
& E_{c 0, e f}=\text { módulo de elasticidade efetivo } \\
& I_{2}=\text { momento de inércia do eixo de menor inércia } \\
& L_{1}=\text { comprimento de flabagem }
\end{aligned}
$$

Dados os valores de $\alpha_{\mathrm{m}}$ pela Tabela 2.1: 
Tabela 2.1 - Representação da Tabela 17 da NBR 7190/1997

\begin{tabular}{|c|c|}
\hline $\mathrm{m}$ & $\alpha_{\mathrm{m}}$ \\
\hline 2 & 1 \\
3 & 1,5 \\
4 & 1,7 \\
5 & 1,8 \\
$\infty$ & 2 \\
\hline
\end{tabular}

\subsubsection{Normas internacionais}

$\mathrm{O}$ valor $\mathrm{N}_{\mathrm{d}} / 150$ representa metade dos valores adotados pelas, mais relevantes, normas internacionais.

O Eurocode 5 adota os valores: $\mathrm{N}_{\mathrm{d}} / 50$ para madeira serrada e $\mathrm{N}_{\mathrm{d}} / 80$ para madeira laminada colada.

Documentos americanos do TPI (Truss Plate Institute) indicam a adoção de 2\% da força máxima (N/50), baseado no trabalho de William Zuk (1956).

A norma chilena, NCh 1198 "Construciones em madera”, de 2006, adota os valores N/50 para madeira serrada e N/100 para madeira laminada colada. A Figura 2.5 foi retirada da norma chilena e mostra a disposição dos esforços no contraventamento.

UNDERWOOD (2000), baseando-se nos resultados de seu estudo, propõe valores da ordem de 3,1\% da força máxima na treliça (N/32).

A norma sul-africana SABS 0163 “The structural use of timber” adota a Expressão 2.3, como mostrado por UNDERWOOD (2000):

$$
P_{L}=\frac{0,10 \cdot P_{A}}{(N+1)}
$$

Onde:

$$
\begin{aligned}
& P_{L}=\text { força no contraventamento } \\
& P_{A}=\text { força axial no elemento contraventado devido à carga permanente } \\
& N=\text { número de restrições ao longo do elemento contraventado }
\end{aligned}
$$




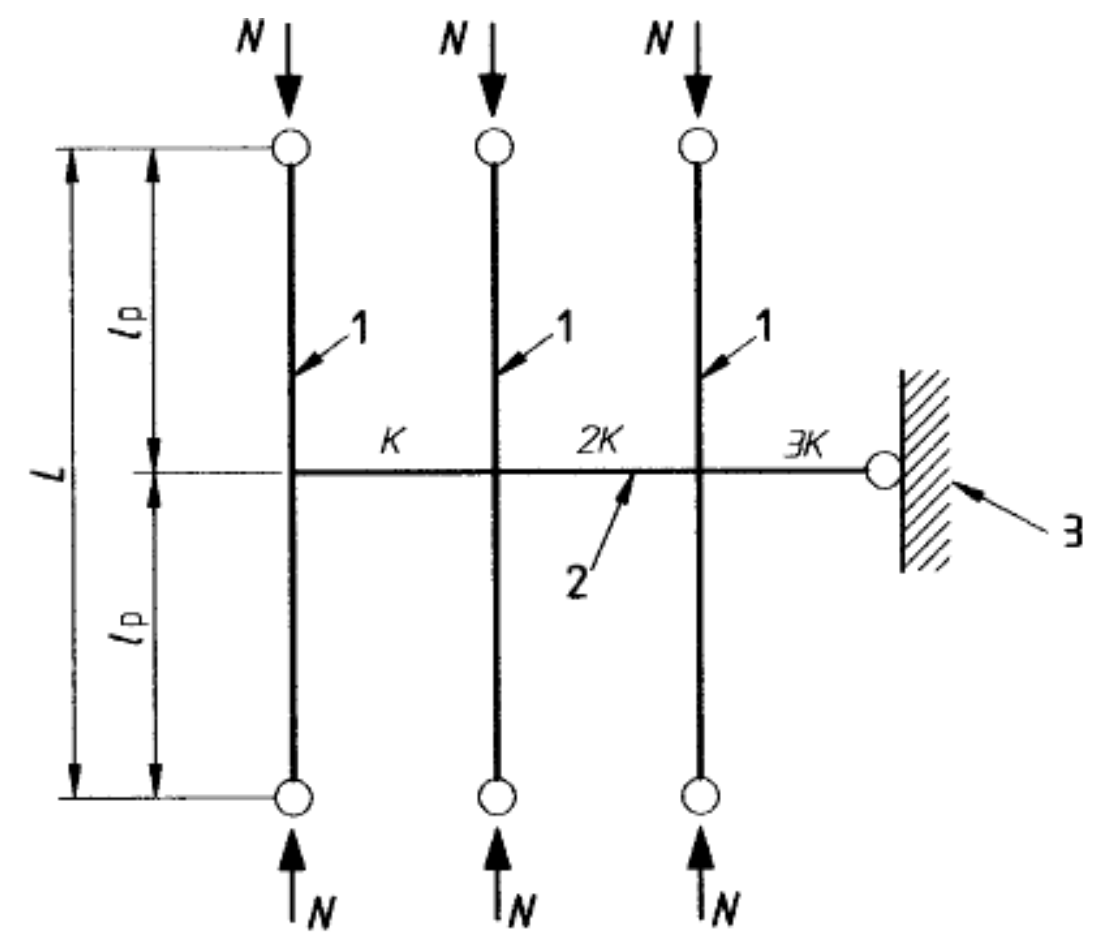

1 Elemento comprimido

2 Puntales

3 Apoyo del puntal constituido por ejemplo, por un sistema de arriostramiento, muros de albañilería, elementos de. hormigón armado o similares

\section{Apuntalamiento individual de piezas comprimidas}

Figura 2.5 - Esforços no contraventamento de peças comprimidas da norma chilena NCh 1198 de 2006

CORDOVIL (2000), em seu estudo, observa os valores mínimos das forças em contraventamento das normas internacionais e demonstra que a norma brasileira prevê uma rigidez, das barras de contraventamento, duas vezes maior que o N/150, como mostrado a seguir.

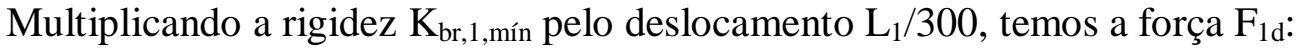

$$
F_{1 d}=\frac{L_{1}}{300} \cdot K_{b r, 1, \text { min }}
$$

Logo:

$$
F_{1 d}=\frac{L_{1}}{300} \cdot 2 \alpha_{m} \frac{\left(\pi^{2} \cdot E_{c 0, e f} \cdot I_{2}\right)}{L_{1}^{3}}
$$


Simplificando a expressão, temos:

$$
F_{1 d}=\frac{1}{150} \cdot \alpha_{m} \frac{\left(\pi^{2} \cdot E_{c 0, e f} \cdot I_{2}\right)}{L_{1}^{2}}
$$

O último termo da equação trata-se da força crítica de estabilidade à compressão, denominada força crítica de Euler:

$$
F_{E}=\frac{\left(\pi^{2} \cdot E_{c 0, e f} \cdot I_{2}\right)}{L_{1}^{2}}
$$

Por fim, resta a equação:

$$
\begin{aligned}
& F_{1 d}=\frac{1}{150} \cdot \alpha_{m} \cdot F_{E} \\
& F_{1 d}=\alpha_{m} \cdot \frac{F_{E}}{150}
\end{aligned}
$$

Assim, coclui-se que o valor para $\alpha_{\mathrm{m}}$ da Tabela 2.1 considerado pela norma foi 1 , e não o mais conservador 2 que resultaria em:

$$
F_{1 d}=2 \cdot \frac{F_{E}}{150}=\frac{F_{E}}{75}
$$

\subsubsection{Estabilidade global de elementos estruturais em paralelo}

Para treliças o sistema de contraventamento apresenta-se indispensável, pois são elementos de grande inércia no plano de atuação dos esforços principais, mas pequena inércia na direção transversal - como mostra a Figura 2.6 - onde se pode considerar que cada elemento da treliça trabalha individualmente. Assim, é necessário o contraventamento das peças comprimidas isoladas, das treliças e da estrutura global, de acordo com as considerações de projeto. 

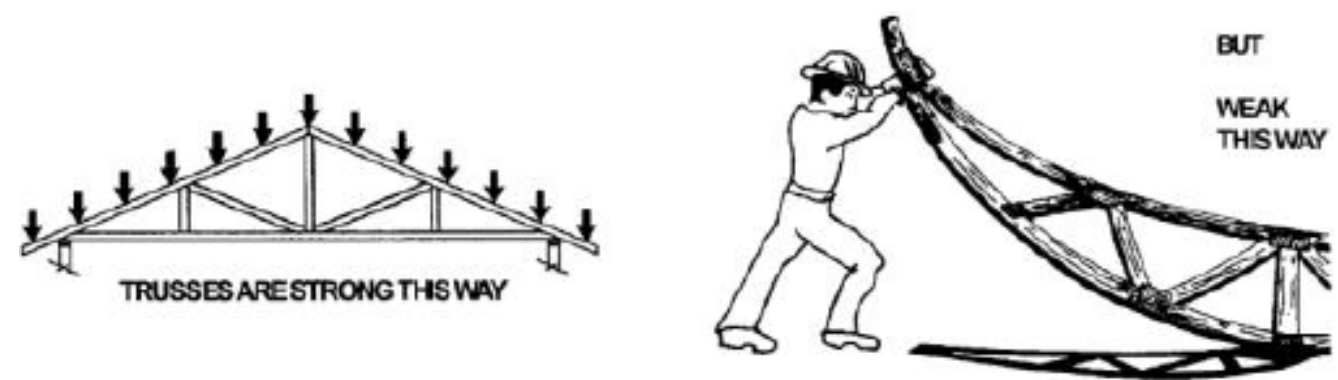

Figura 2.6 - Baixa rigidez lateral das treliças (Fonte: Truss Plate Institute)
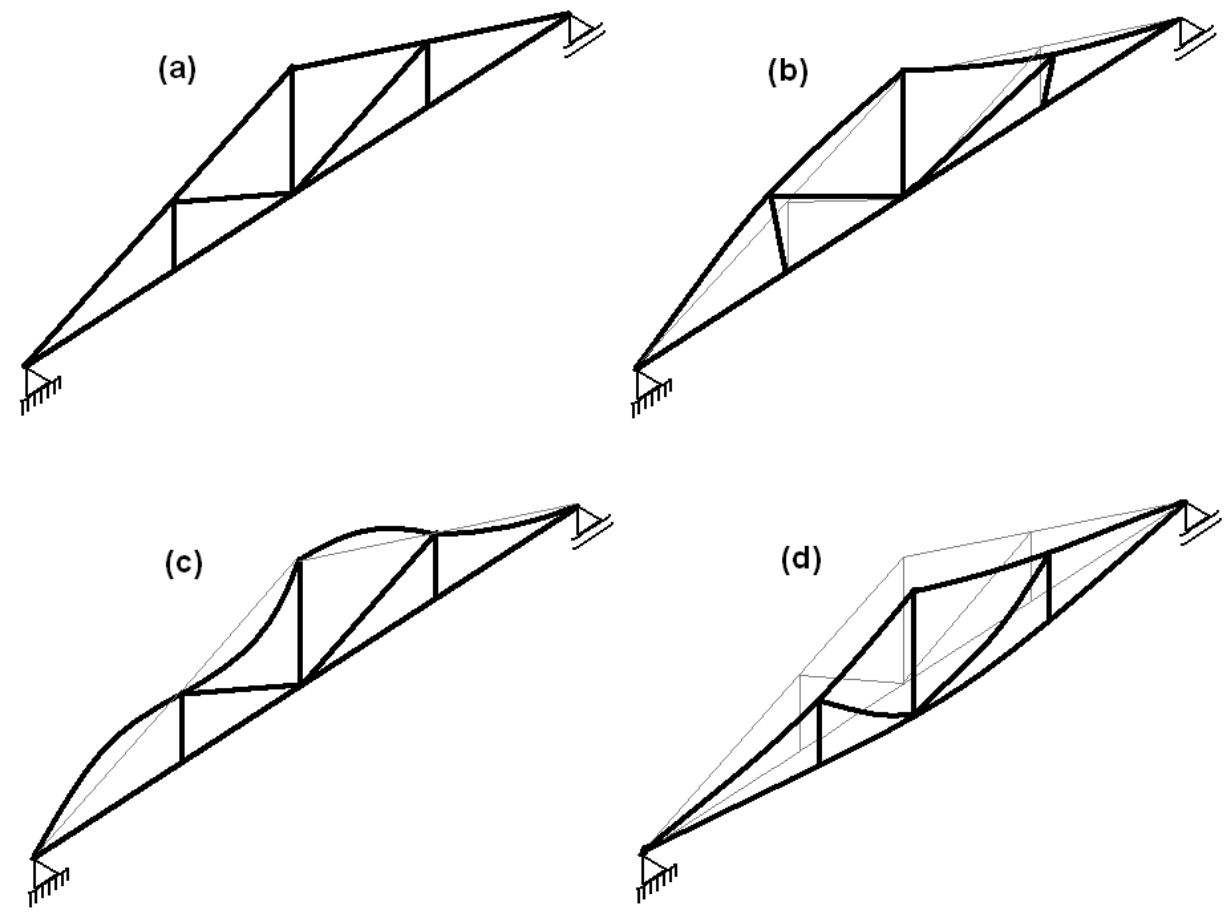

Figura 2.7 - Possíveis casos de instabilidade de uma treliça 


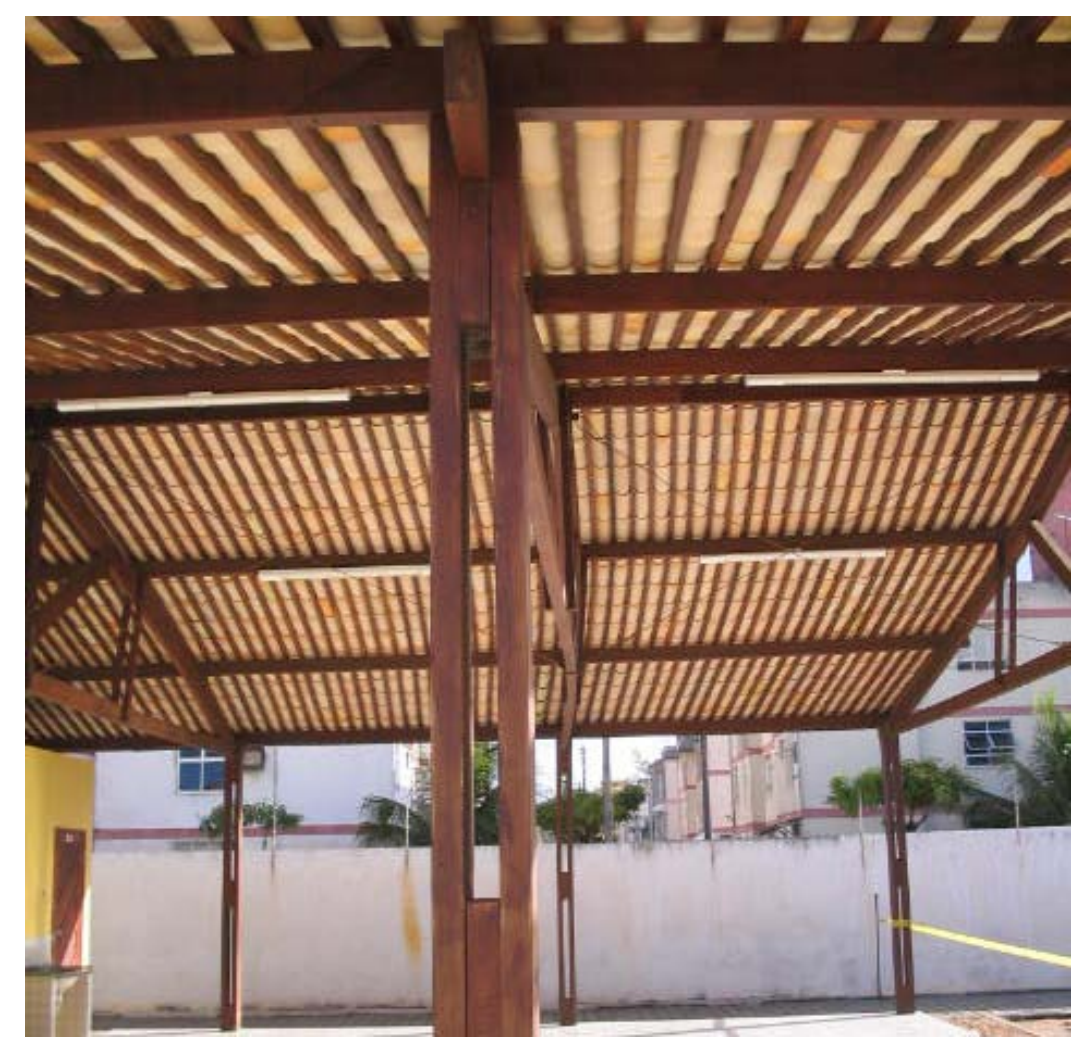

Figura 2.8 - Flambagem lateral da tesoura completa, caso (d) da Figura 2.7

A NBR 7190 de 1997 fixa, no item 7.6.4 Estabilidade global de elementos estruturais em paralelo, as prescrições para contraventamento lateral de um sistema estrutural formado por elementos paralelos como no caso de coberturas de treliças de madeira. O sistema de contraventamento deve ser composto por treliças dispostas perpendicularmente ao plano das estruturas principais, nos planos horizontal, vertical e no plano da cobertura. Este sistema de travamento deve existir nas extremidades da construção e em eventuais posições intermediárias, existindo em um de cada três vãos definidos pelos elementos do sistema principal, não excedendo o espaçamento de 20 metros.

O sistema de contraventamento tem a função de impedir deslocamentos transversais nos nós da estrutura, transferindo os esforços para os nós fixos de apoio. O funcionamento do contraventamento é mostrado na Figura 2.9. 

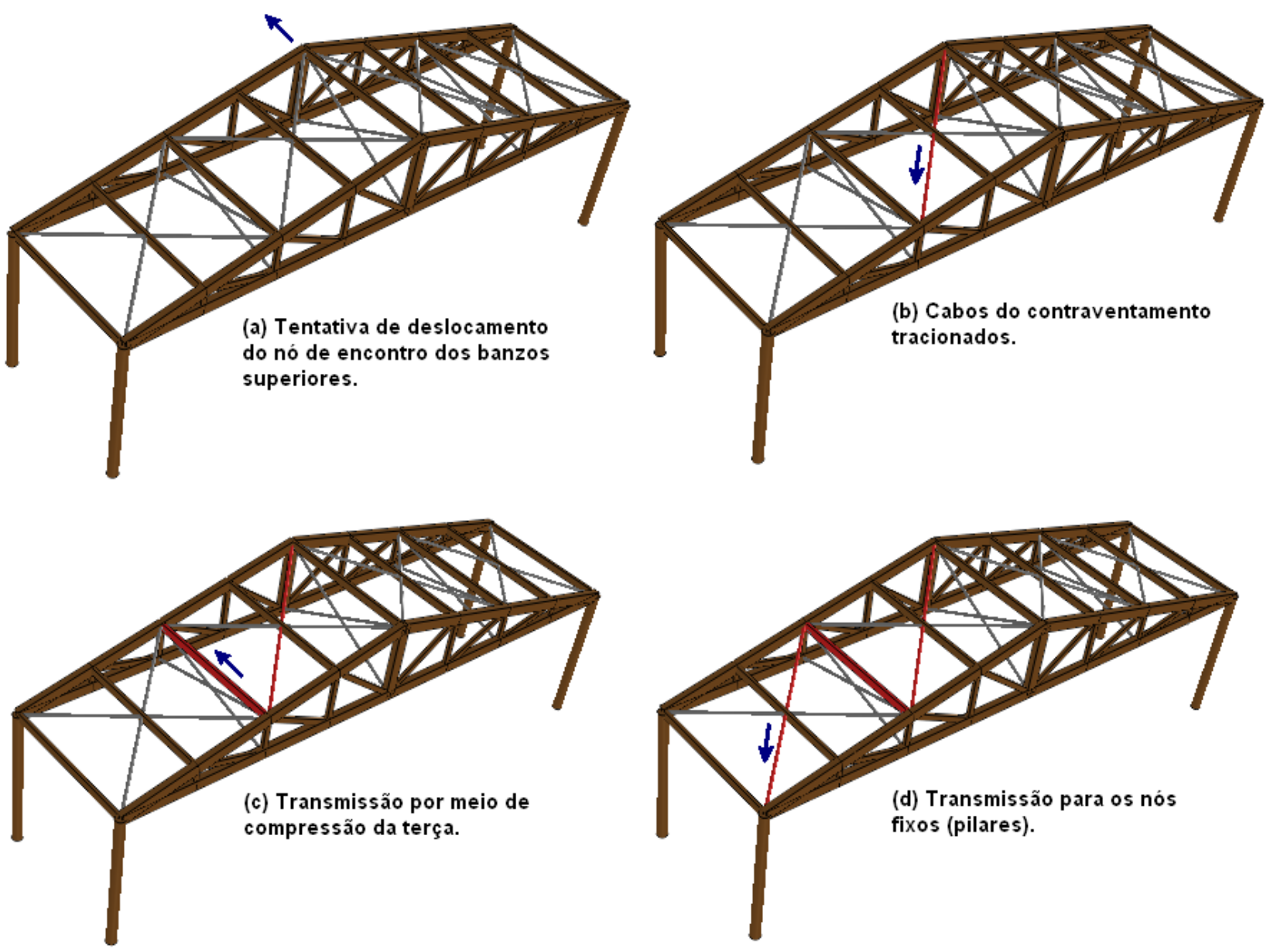

Figura 2.9 - Funcionamento do contraventamento

Em cada nó contraventado, do banzo comprimido do sistema principal, deve ser considerada uma força transversal $F_{1 d}$ de valor $\mathrm{N}_{\mathrm{d}} / 150$, onde $\mathrm{N}_{\mathrm{d}}$ é a resultante das tensões no banzo comprimido, como mostra na Figura 2.10.

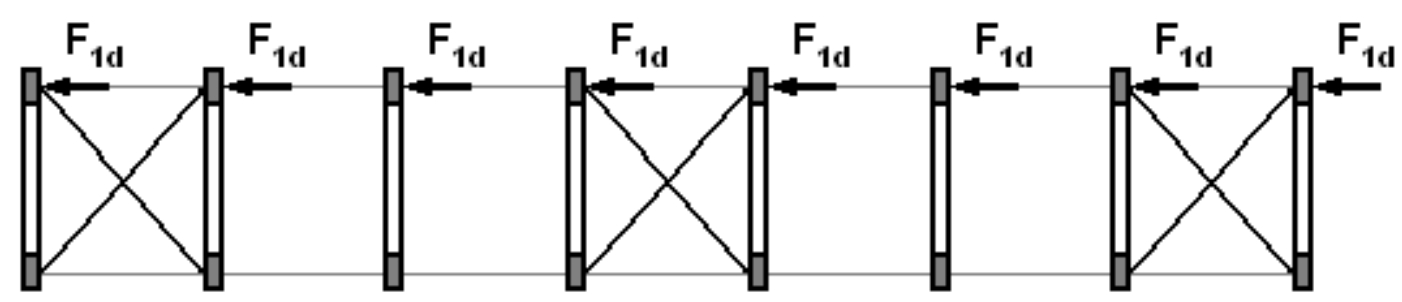

Figura 2.10 - Arranjo vertical de contraventamento 
As estruturas de contraventamento devem resistir a uma força $F_{d}$ de valor maior ou igual a $2 / 3$ da resultante das $n$ forças $F_{1 d}$ existentes no trecho a ser estabilizado, como mostrado na Figura 2.11.

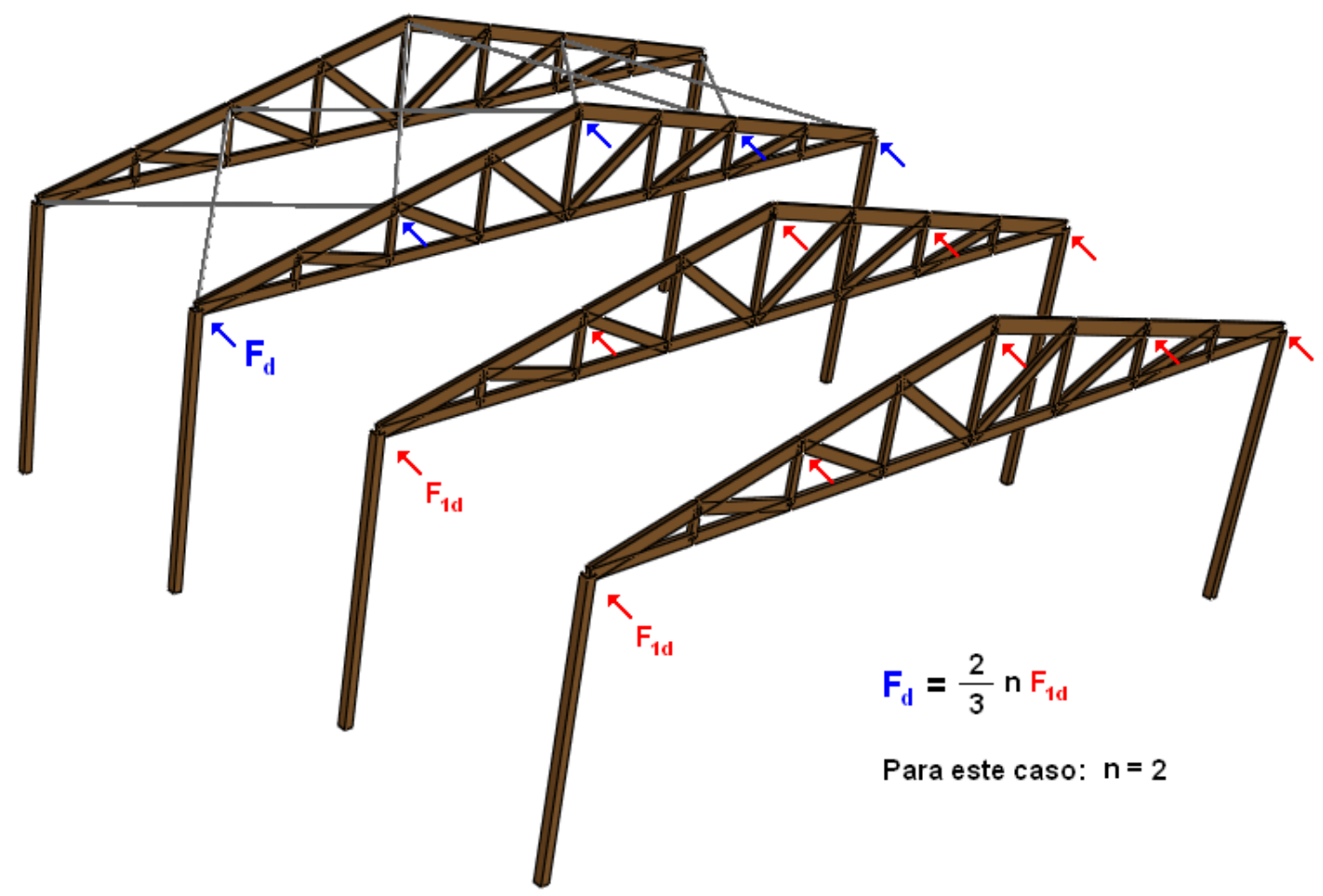

Figura 2.11 - Arranjo de esforços de contraventamento

UNDERWOOD (2000), em seu estudo sobre projeto de contraventamento permanente de treliças de coberturas de madeira, apresenta as Expressões 2.11 e 2.12 da norma sulafricana para contraventamento de treliças de madeira.

$$
P_{L}=\frac{0,10 \cdot P_{A}}{(N+1)}
$$

Onde:

$$
\begin{aligned}
& P_{L}=\text { força em cada elemento de contraventamento lateral } \\
& P_{A}=\text { força axial máxima na treliça devido à carga permanente } \\
& N=\text { número de restrições ao longo da treliça }
\end{aligned}
$$




$$
C_{P L N}=P_{L} \cdot n^{0,7}
$$

Onde:

$$
\begin{aligned}
& P_{L}=\text { força em cada elemento de contraventamento lateral } \\
& n=\text { número de treliças contraventadas }
\end{aligned}
$$

BARALDI (2001) apresenta métodos de cálculo que consideram os painéis de vedação da estrutura como diafragmas que distribuem os esforços horizontais nos pórticos principais formados pelas treliças e pilares. A consideração da ação diafragma fornece valores mais precisos e uma estrutura mais econômica do ponto de vista dos elementos do contraventamento.

Diretrizes para consideração da ação diafragma são encontradas também no Canadian Wood Construction (1986) e na norma americana ANSI/TPI (1995), citados por BARALDI (2001).

Como mostra a Figura 2.12, o modelo de ação diafragma considera os painéis de vedação das paredes e da cobertura como diafragmas que transferem os esforços entre os pórticos principais da estrutura.
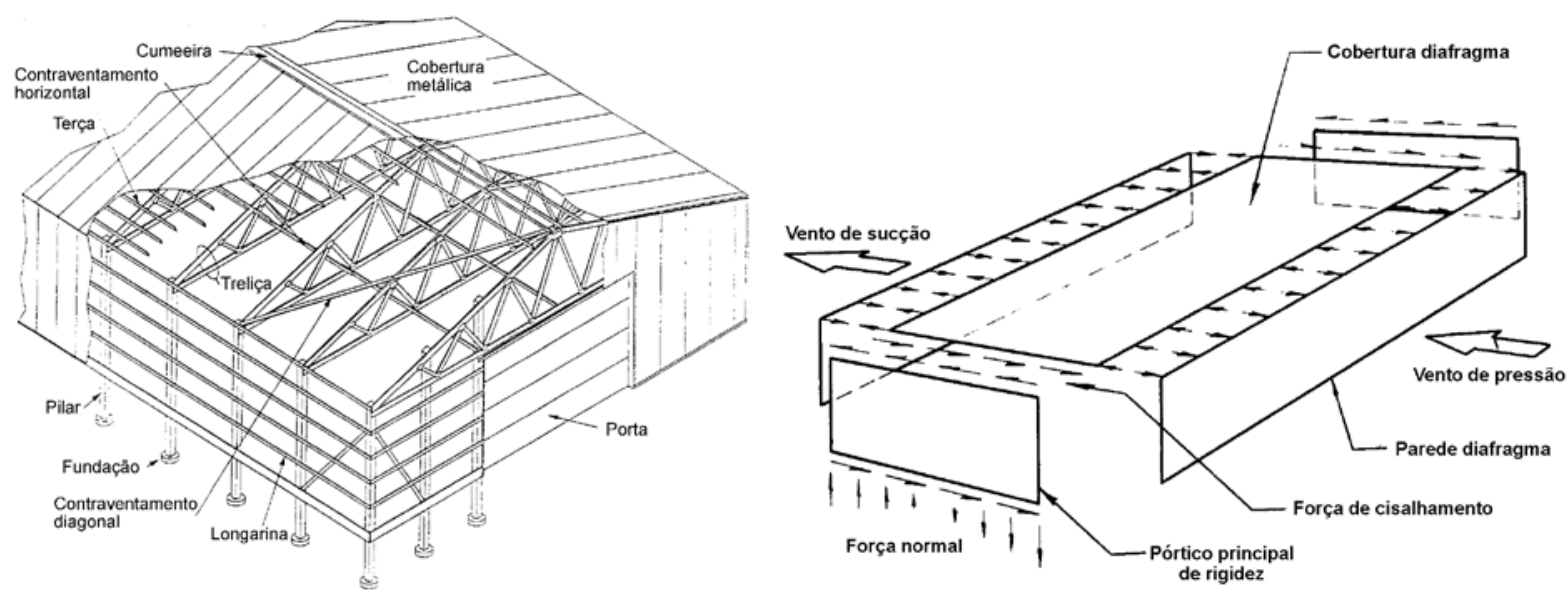

Figura 2.12 - Esquema de ação diaframa. Fonte: BARALDI (2001) 


\subsubsection{Contraventamento como parte da estrutura tridimensional}

A forma de estimar os esforços atuantes nos elementos de contraventamento, como parte da estrutura global, é a análise do modelo tridimensional da estrutura. Neste caso o contraventamento trabalha ajudando a resistir às ações principais atuantes e não apenas aos possíveis deslocamentos fora do plano de maior inércia.

Os valores de $F_{1 d}$ e $F_{d}$ expostos pela norma são valores mínimos, sua utilização é para casos em que não haja uma análise estrutural rigorosa. A forma dessa análise rigorosa não é fixada pela norma, assim, fica a critério do projetista adotar um método de sua preferência que condiz com o conhecimento existente.

Observa-se que o funcionamento tridimensional tem influência significativa nas barras de contraventamento e devem ser analisados e suas forças somadas às devido à instabilidade lateral.

Um dos possíveis métodos foi abordado por ROCCO LAHR et al. (2008). No estudo são analisados os esforços atuantes nas tesouras e no contraventamento de um galpão industrial de madeira com treliças tipo Pratt de duas águas em telha metálica com dimensões em planta de 13,00 m x 40,00 m, colunas de madeira roliça com altura de 4,00 m e altura da tesoura $1,00 \mathrm{~m}$. 


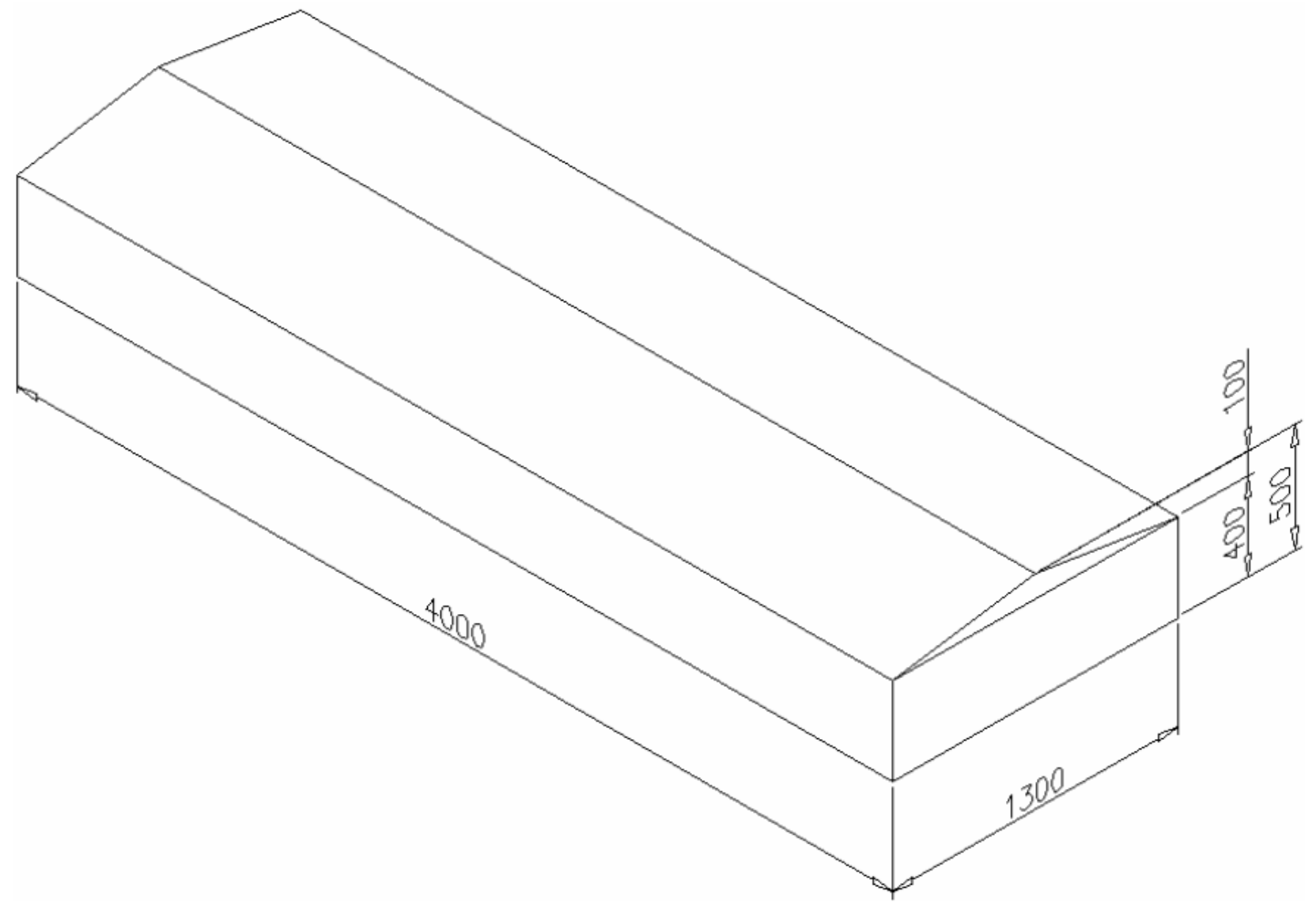

Figura 2.13 - Desenho esquemático do modelo estudado por ROCCO LAHR et al. (2008)

São analisados os esforços de um modelo plano com cargas nodais calculado no programa Ftool - programa desenvolvido pela PUC-Rio, disponibilizado gratuitamente - e um modelo tridimensional de todo o galpão modelado e analisado no SAP 2000. Na análise tridimensional são aplicadas as ações de projeto calculadas para uma tesoura, da mesma forma da tesoura plana, e dispostas as 11 tesouras do galpão completo com correspondentes pilares e barras de contraventamento.

Na Tabela 2.2 observa-se, nos valores obtidos no trabalho, que houve uma redução considerável dos esforços axiais na treliça no modelo tridimensional em relação à análise plana. Porém, os valores observados nas peças de contraventamento, mostrados na Tabela 2.3, foram bem superiores aos mínimos previstos por norma. 
Tabela 2.2 - Comparação de esforços obtidos, nos modelos tri e bidimensionais, por ROCCO LAHR et al. (2008)

\begin{tabular}{|c|c|c|c|c|c|c|}
\hline \multicolumn{7}{|c|}{ ENVOLTÓRIA DOS ESFORÇOS } \\
\hline \multirow{2}{*}{ Elemento } & \multicolumn{2}{|c|}{ SAP 2000 } & \multicolumn{2}{c|}{ FTOOL } & \multicolumn{1}{c|}{ COMPARAÇÃO } \\
\cline { 2 - 7 } & Axial [kN] & Axial [kN] & Axial [kN] & Axial [kN] & Compr. & Tração \\
\hline $\mathbf{1}$ & 100,98 & $-74,51$ & 126,24 & $-80,91$ & $25,01 \%$ & $8,59 \%$ \\
\hline $\mathbf{2}$ & 100,81 & $-74,36$ & 127,36 & $-81,10$ & $26,34 \%$ & $9,06 \%$ \\
\hline $\mathbf{3}$ & 85,42 & $-64,19$ & 108,90 & $-70,75$ & $27,49 \%$ & $10,22 \%$ \\
\hline $\mathbf{4}$ & 70,51 & $-52,52$ & 90,28 & $-60,65$ & $28,04 \%$ & $15,48 \%$ \\
\hline $\mathbf{5}$ & 65,52 & $-94,61$ & 79,62 & $-124,61$ & $21,52 \%$ & $31,71 \%$ \\
\hline $\mathbf{6}$ & 55,47 & $-79,38$ & 69,16 & $-105,21$ & $24,68 \%$ & $32,54 \%$ \\
\hline $\mathbf{7}$ & 44,75 & $-64,62$ & 58,88 & $-85,53$ & $31,58 \%$ & $32,36 \%$ \\
\hline $\mathbf{8}$ & 32,74 & $-47,35$ & 47,53 & $-64,20$ & $45,17 \%$ & $35,59 \%$ \\
\hline $\mathbf{9}$ & 10,35 & $-15,69$ & 12,67 & $-19,96$ & $22,42 \%$ & $27,21 \%$ \\
\hline $\mathbf{1 0}$ & 12,45 & $-15,88$ & 14,92 & $-21,16$ & $19,84 \%$ & $33,25 \%$ \\
\hline $\mathbf{1 1}$ & 13,46 & $-19,35$ & 16,80 & $-24,00$ & $24,81 \%$ & $24,03 \%$ \\
\hline $\mathbf{1 2}$ & 4,01 & $-2,10$ & 4,89 & $-2,53$ & $21,95 \%$ & $20,48 \%$ \\
\hline $\mathbf{1 3}$ & 6,29 & $-3,04$ & 7,76 & $-3,68$ & $23,37 \%$ & $21,05 \%$ \\
\hline $\mathbf{1 4}$ & 9,06 & $-5,57$ & 10,73 & $-5,71$ & $18,43 \%$ & $2,51 \%$ \\
\hline $\mathbf{1 5}$ & 1,28 & $-1,95$ & 0,00 & 0,00 & - & - \\
\hline
\end{tabular}

Tabela 2.3 - Esforços obtidos nas demais peças do modelo tridimensional

\begin{tabular}{|c|c|c|c|c|}
\hline \multicolumn{5}{|c|}{ ENVOLTÓRIA DE ESFORÇOS } \\
\hline Elemento & Axial [kN] & Axial [kN] & Momento [kN.m] & Cortante [kN] \\
\hline COLUNAS & 15,83 & $-16,36$ & 840,00 & 6,80 \\
\hline VIGAS & 5,83 & $-5,24$ & 22,18 & - \\
\hline C.H. & 2,14 & $-6,06$ & - & - \\
\hline C.V. & 5,95 & $-4,14$ & - & - \\
\hline
\end{tabular}

C.H. = contraventamento horizontal

C.V. = contraventamento vertical 


\subsection{Considerações finais}

O método - de estimativa da força no contraventamento - indicado pela NBR 7190 (1997) apresenta valores inferiores aos apresentados pelas normas internacionais.

Os métodos pesquisados são baseados na instabilidade lateral dos elementos com imperfeição geométrica de curvatura da ordem de L/300 e L/200. A análise tridimensional do modelo estrutural, que consideram o funcionamento do contraventamento como parte da estrutura global, resulta em valores mais altos que os estimados pelo método da norma brasileira. Assim, pode-se estar subestimando as forças atuantes no contraventamento.

São necessários estudos do comportamento tridimensional da estrutura para que sejam considerados valores mais seguros, para casos de ausência de uma análise estrutural rigorosa. 


\section{ANÁLISE NUMÉRICA}

Neste capítulo são apresentados os procedimentos para realização do trabalho. Trata-se da modelagem numérica, de galpões de estruturas treliçadas de madeira do tipo tesouras triangulares, por meio de ferramentas computacionais de análise estrutural. A seguir são descritos: programas de análise estrutural, geometrias, condições de contorno, propriedades das barras e ações consideradas na modelagem.

\subsection{Programas computacionais}

Para estimar os esforços atuantes nos elementos estruturais dos galpões, foram utilizados dois programas computacionais de análise estrutural. O AcadFrame e o STRAP, ambos utilizam o Método dos Elementos Finitos para estimativa dos esforços em cada elemento da estrutura.

\subsubsection{AcadFrame}

O AcadFrame é uma ferramenta computacional, desenvolvida no Departamento de Engenharia de Estruturas da Escola de Engenharia de São Carlos, que utiliza o Método dos Elementos Finitos Posicional para análise de pórticos e treliças planas incluindo não linearidade geométrica, efeito de temperatura e combinação de ações externas. Sua versão acadêmica é disponibilizada gratuitamente no endereço eletrônico: http://www.set.eesc.usp.br/acadframe/.

A utilização do AcadFrame teve por finalidade apenas a conferência dos resultados dos modelos bidimensionais obtidos no programa STRAP, uma que vez que o AcadFrame foi desenvolvido segundo os métodos numéricos estudados. 


\subsubsection{STRAP}

Os modelos tridimensionais foram confeccionados no STRAP (Structural Analysis Programs). O programa utiliza o Método dos Elementos Finitos para análises de esforços em estruturas. Utilizou-se a versão STRAP 2008, e os modelos constituídos de elementos de barras e a análise dos esforços realizada de forma linear.

\subsection{Geometrias dos modelos}

Os modelos computacionais foram analisados com dois tipos de geometrias, sendo: a treliça plana isostática (forma clássica) e o modelo tridimensional formado pelos pórticos, terças e contraventamentos.

Todos os modelos apresentam cobertura com $25 \%$ de inclinação. As treliças são divididas em quadros com 1,50 metros de distância entre montantes. Os vãos das treliças são de 12, 18 e 24 metros e os pilares são de alturas 3, 4 e 6 metros. As ações atuantes variam com a classe de madeira, o tipo de telha e a ação estática de vento de acordo com as dimensões de cada galpão, que estão descritas no item 3.5.

Além da variação nas dimensões, foram estudados dois tipos de tesouras, a Howe e a Pratt, que se diferenciam pelo sentido de inclinação das escoras. Sentido de inclinação este que inverte a natureza de esforços de tração e compressão.

A seguir são listados os modelos, suas características e finalidades. 


\subsubsection{Treliças planas}

O modelo de treliça plana, o mais simples e mais utilizado na prática, fornece os valores usuais de projeto, base para cálculo dos esforços de projeto nas peças de contraventamento segundo os métodos estudados: NBR 7190 (1997), EUROCODE 5, UNDERWOOD (2000) e SABS 0163.

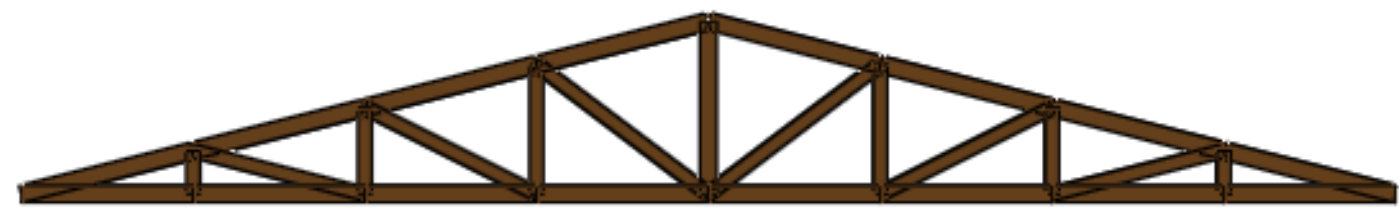

Figura 3.1 - Treliça plana tipo Howe

São 11 modelos de treliças para cada tipo (Howe e Pratt) e classe (C30 e C50) estudados, sendo 44 modelos de treliças. Também são analisados o tipo Pratt C50 considerando telhas metálicas e 7 modelos tipo Pratt C50 considerando telhas cerâmicas, para as treliças até 18 metros e espaçamento igual a 3 metros. Totalizando 61 modelos.

Tabela 3.1 - Geometrias dos modelos de treliças

\begin{tabular}{|c|c|c|c|}
\hline Treliça & Vão (m) & z (altura) & $\begin{array}{l}\text { e (vão entre } \\
\text { treliças) }\end{array}$ \\
\hline 1 & \multirow{3}{*}{12} & 3 & \multirow{6}{*}{3} \\
\hline 2 & & 4 & \\
\hline 3 & & 6 & \\
\hline 4 & \multirow{4}{*}{18} & 3 & \\
\hline 5 & & 4 & \\
\hline 6 & & 6 & \\
\hline 7 & & 6 & 4 \\
\hline 8 & \multirow{4}{*}{24} & 3 & \multirow{3}{*}{3} \\
\hline 9 & & 4 & \\
\hline 10 & & 6 & \\
\hline 11 & & 6 & 4 \\
\hline
\end{tabular}




\subsubsection{Galpões completos}

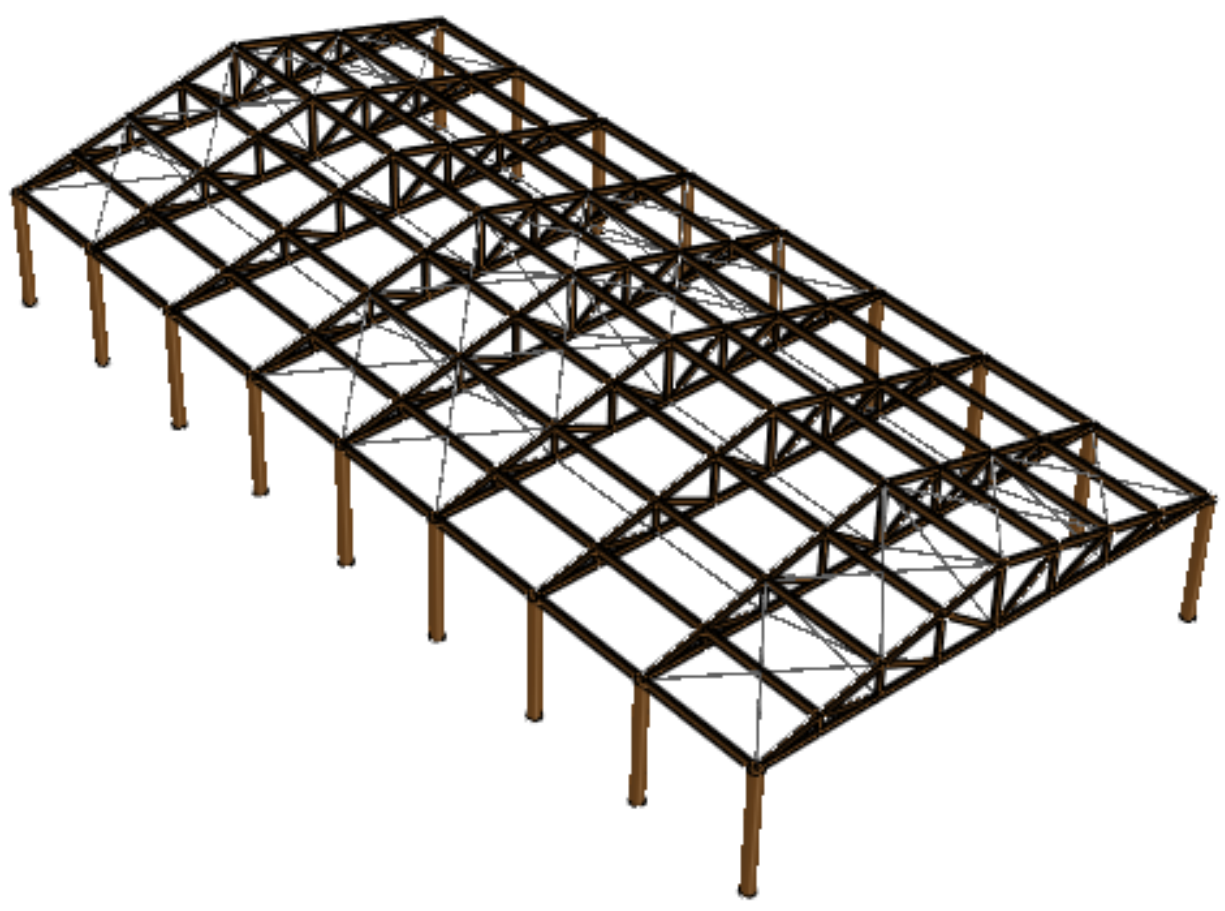

Figura 3.2 - Estrutura completa de galpão com treliça tipo Howe e contraventamento de barras de aço

Foram consideradas 21 geometrias base de galpões industriais de estruturas de madeira. Os modelos foram analisados para os tipos Howe e Pratt, as classes C30 e C50, o contraventamento com todos os nós da treliça contraventados e com um a cada dois nós da treliça contraventado, e os modelos tipo Pratt de classe C50 foram analisados também com contraventamento de madeira, telhas metálicas e telhas cerâmicas, o que totaliza 238 modelos de galpões.

Os valores obtidos nos modelos tridimensionais têm por finalidade analisar o funcionamento em conjunto do contraventamento com a estrutura.

Ao todo foram analisados 299 modelos, sendo o menor (Treliça plana $\mathrm{L}=12 \mathrm{~m} \mathrm{~h}=1,5 \mathrm{~m}$ z=3m) constituído por 16 nós e 29 barras, e o maior (Galpão 20) constituído por 1122 nós e 3667 barras. Observação: $L=$ vão, $h=$ altura central, $\mathrm{z}=$ altura do pilar. 


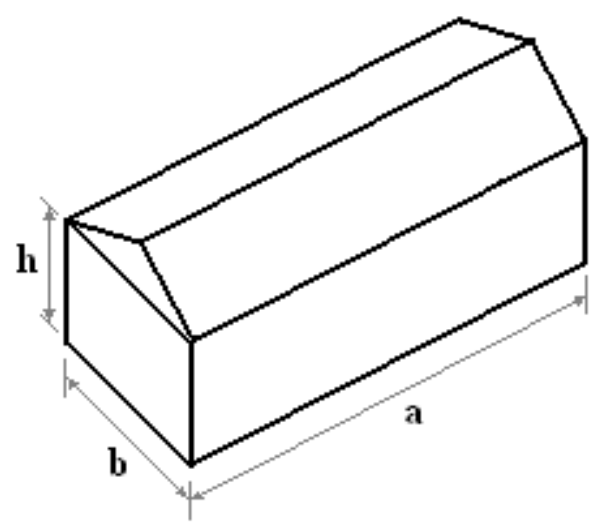

Figura 3.3 - Desenho esquemático das dimensões de um galpão

Tabela 3.2 - Dimensões dos galpões estudados (unidades em metros)

\begin{tabular}{|c|c|c|c|}
\hline Galpão & $\begin{array}{c}\text { Planta } \\
\text { b x a }\end{array}$ & z (altura) & $\begin{array}{c}\text { e (vão entre } \\
\text { treliças) }\end{array}$ \\
\hline 1 & \multirow{3}{*}{$12 \times 24$} & 3 & \multirow{12}{*}{3} \\
\hline 2 & & 4 & \\
\hline 3 & & 6 & \\
\hline 4 & \multirow{3}{*}{12 x 48} & 3 & \\
\hline 5 & & 4 & \\
\hline 6 & & 6 & \\
\hline 7 & \multirow{3}{*}{$18 \times 24$} & 3 & \\
\hline 8 & & 4 & \\
\hline 9 & & 6 & \\
\hline 10 & \multirow{4}{*}{18 x 48} & 3 & \\
\hline 11 & & 4 & \\
\hline 12 & & 6 & \\
\hline 13 & & 6 & 4 \\
\hline 14 & \multirow{4}{*}{$24 \times 48$} & 3 & \multirow{3}{*}{3} \\
\hline 15 & & 4 & \\
\hline 16 & & 6 & \\
\hline 17 & & 6 & 4 \\
\hline 18 & \multirow{4}{*}{$24 \times 96$} & 3 & \multirow{3}{*}{3} \\
\hline 19 & & 4 & \\
\hline 20 & & 6 & \\
\hline 21 & & 6 & 4 \\
\hline
\end{tabular}




\subsection{Propriedades das barras}

As treliças e os galpões foram analisados considerando as classes de resistência C30 e C50, por serem classes usuais para os vãos adotados.

A rigidez de cada barra dos modelos é considerada pela seção e pelo módulo de elasticidade parametrizado no programa. Os valores para peças de madeira são estimados a partir dos valores da Tabela 3.4 - estudados no Laboratório de Madeira e Estruturas de Madeira LaMEM e publicados por CALIL JR. (2008) - e para obtenção do valor de projeto do módulo de elasticidade efetivo $E_{c 0, \text { ef }}$ são multiplicados pelo coeficiente de correção $K_{\text {mod }}$ igual a 0,56. Para barras ou cabos de aço foi considerado o módulo de elasticidade de 195.000 MPa.

Tabela 3.3 - Classes de resistência das coníferas. (Fonte: CALIL JR., 2008)

\begin{tabular}{|c|c|c|c|c|c|}
\hline \multicolumn{6}{|c|}{ CLASSES DE RESISTÊNCIA DAS CONÍFERAS (U = 12\%) } \\
\hline Classe & $\begin{array}{c}\mathrm{f}_{\mathrm{c} 0, \mathrm{k}} \\
(\mathrm{MPa})\end{array}$ & $\begin{array}{c}\mathrm{f}_{\mathrm{vk}} \\
(\mathrm{MPa})\end{array}$ & $\begin{array}{c}\mathrm{E}_{\mathrm{c} 0, \mathrm{~m}} \\
(\mathrm{MPa})\end{array}$ & $\begin{array}{c}\rho_{\text {bas,m }} \\
\left(\mathrm{kgf} / \mathrm{m}^{3}\right)\end{array}$ & $\begin{array}{c}\rho_{\mathrm{ap}} \\
\left(\mathrm{kgf} / \mathrm{m}^{3}\right)\end{array}$ \\
\hline C20 & 20 & 4 & 3500 & 400 & 500 \\
\hline C25 & 25 & 5 & 8500 & 450 & 550 \\
\hline C30 & 30 & 6 & 14500 & 500 & 600 \\
\hline
\end{tabular}

Tabela 3.4 - Classes de resistência das dicotiledôneas. (Fonte: CALIL JR., 2008)

CLASSES DE RESISTÊNCIA DAS DICOTILEDÔNEAS (U = 12\%)

\begin{tabular}{|c|c|c|c|c|c|}
\hline Classe & $\begin{array}{c}\mathrm{f}_{\mathrm{c} 0, \mathrm{k}} \\
(\mathrm{MPa})\end{array}$ & $\begin{array}{c}\mathrm{f}_{\mathrm{vk}} \\
(\mathrm{MPa})\end{array}$ & $\begin{array}{c}\mathrm{E}_{\mathrm{c} 0, \mathrm{~m}} \\
(\mathrm{MPa})\end{array}$ & $\begin{array}{c}\rho_{\text {bas,m }} \\
\left(\mathrm{kgf} / \mathrm{m}^{3}\right)\end{array}$ & $\begin{array}{c}\rho_{\mathrm{ap}} \\
\left(\mathrm{kgf} / \mathrm{m}^{3}\right)\end{array}$ \\
\hline $\mathbf{C 2 0}$ & 20 & 4 & 9500 & 500 & 650 \\
\hline $\mathbf{C 3 0}$ & 30 & 5 & 14500 & 650 & 800 \\
\hline $\mathbf{C 4 0}$ & 40 & 6 & 19500 & 750 & 950 \\
\hline $\mathbf{C 5 0}$ & 50 & 7 & 22000 & 770 & 970 \\
\hline $\mathbf{C 6 0}$ & 60 & 8 & 24500 & 800 & 1000 \\
\hline
\end{tabular}


Onde:

$$
\begin{aligned}
& f_{c 0, k}=\text { resistência característica à compressão } \\
& f_{v k}=\text { resistência característica ao cisalhamento } \\
& E_{c 0, m}=\text { módulo de elasticidade médio na direção paralela às fibras } \\
& \rho_{\text {bas,m }}=\text { densidade básica } \\
& \rho_{a p}=\text { densidade aparente } \\
& U=\text { teor de umidade da madeira }
\end{aligned}
$$

Nas propriedades também é determinado o peso específico do material, para aplicação

\begin{tabular}{|c|c|c|c|c|c|c|c|c|}
\hline \multicolumn{9}{|c|}{ SEÇÕES DAS BARRAS DA TRELIÇA (cm) } \\
\hline \multirow[b]{3}{*}{ Classe } & \multirow{3}{*}{$\begin{array}{l}\text { Vão } \\
\text { da } \\
\text { treliça } \\
\text { (m) }\end{array}$} & \multirow{3}{*}{$\begin{array}{l}\text { Espaçamento } \\
\text { entre treliças } \\
\text { (m) }\end{array}$} & \multicolumn{6}{|c|}{ Tipo de telha } \\
\hline & & & \multicolumn{2}{|c|}{ Metálicas } & \multicolumn{2}{|c|}{ Fibrocimento } & \multicolumn{2}{|c|}{ Cerâmicas } \\
\hline & & & Banzos & $\begin{array}{c}\text { Montantes } \\
\text { e } \\
\text { diagonais }\end{array}$ & Banzos & $\begin{array}{c}\text { Montantes } \\
\text { e } \\
\text { diagonais }\end{array}$ & Banzos & $\begin{array}{l}\text { Montantes } \\
\text { e diagonais }\end{array}$ \\
\hline \multirow{5}{*}{ C30 } & 12 & 3 & & & $12 \times 15$ & $6 \times 10$ & & \\
\hline & \multirow{2}{*}{18} & 3 & & & $12 \times 20$ & $6 \times 10$ & & \\
\hline & & 4 & & & $12 \times 30$ & $6 \times 12$ & & \\
\hline & \multirow{2}{*}{24} & 3 & & & $20 x 20$ & $6 \times 20$ & & \\
\hline & & 4 & & & $20 x 20$ & $6 \times 25$ & & \\
\hline \multirow{5}{*}{ C50 } & 12 & 3 & $12 \times 12$ & $6 \times 6$ & $12 \times 12$ & $6 \times 6$ & $12 \times 15$ & $6 \times 6$ \\
\hline & \multirow{2}{*}{18} & 3 & $12 \times 12$ & $6 \times 10$ & $12 \times 15$ & $6 \times 10$ & $12 \times 25$ & $6 \times 10$ \\
\hline & & 4 & $12 \times 15$ & $6 \times 10$ & $12 \times 20$ & $6 \times 10$ & & \\
\hline & \multirow{2}{*}{24} & 3 & $12 \times 20$ & $6 \times 15$ & 12x25 & $6 \times 12$ & & \\
\hline & & 4 & $12 \times 25$ & $6 \times 20$ & $12 \times 30$ & $6 \times 15$ & & \\
\hline
\end{tabular}
do peso próprio da estrutura nos modelos computacionais.

As seções do banzo superior foram dimensionadas considerando o comprimento de flambagem de três metros e dez centímetros, referente a dos quadros da treliças, supondo o contraventamento de um a cada dois nós (um xis a cada duas terças).

Tabela 3.5 - Dimensões das seções das barras da treliça 
Para o modelo com barras de contraventamento de madeira foi adotada a seção de 6x15 centímetros.

\subsection{Condições de contorno}

Nos modelos foram consideradas todas as ligações entre barras rotuladas, como considerado na análise clássica.

A forma usual de análise estrutural das treliças de madeira considera todas as ligações entre barras articuladas e os apoios sendo o primeiro uma rótula e o segundo um apoio de primeiro grau com restrição de deslocamento vertical. Porém, na realidade a maioria das ligações usuais apresenta certa restrição à rotação, funcionando, portanto, como ligações semi-rígidas. Outro ponto que oferece restrição à rotação é a continuidade das peças constituintes dos banzos. Estas aproximações de modelos não apresentam influência significante para o caso aqui estudado, portanto não serão considerados nos modelos.

Nos modelos em que as barras de contraventamento são de aço, consideram-se resistentes apenas a esforços de tração devido à alta esbeltez das mesmas e do uso de cabos na prática de execução. Todas as barras constituintes do contraventamento são bi-rotuladas, inclusive as terças.

Os apoios dos pórticos são representados por engastamento nas bases dos pilares.

\subsection{Ações atuantes}

As ações consideradas nos modelos computacionais buscam representar os possíveis casos mais desfavoráveis na estrutura em serviço. São considerados: o peso próprio da estrutura segundo a NBR 7190 (1997); o peso do telhado; e o vento representado pelas ações estáticas dos ventos segundo a NBR 6123 (1988). 


\subsubsection{Peso próprio}

O peso próprio é considerado distribuído linearmente em cada elemento, de acordo com peso específico das classes de resistência consideradas e das dimensões de cada barra previamente parametrizados, aplicado na direção vertical.

Como exemplo, a Figura 3.4 mostra o carregamento de peso próprio para uma tesoura de madeira dicotiledônea de classe C50, peso específico de $9,7 \mathrm{kN} / \mathrm{m}^{3}$, representados por cargas linearmente distribuídas e as cargas concentradas representando as terças. Os valores das propriedades são indicados pela Tabela 3.3 e Tabela 3.4, extraídas de CALIL JR. (2008).

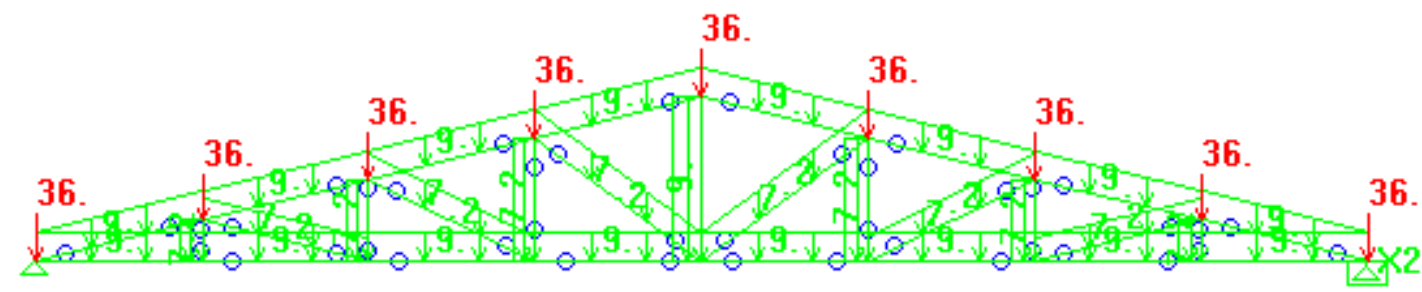

Figura 3.4 - Carregamento do peso próprio (em daN e m) da tesoura tipo C50 (970 daN/m³) e das terças representadas pelas cargas concentradas

\subsubsection{Telhas}

Para os modelos que variam classe de resistência de tipo de material do contraventamento foi considerado o carregamento de telha referente à telha de fibrocimento $\left(180 \mathrm{~N} / \mathrm{m}^{2}\right)$ por ser bastante usada na prática e apresentar um valor de peso médio entre as telhas metálicas e cerâmicas. O valor da carga considerado para a telha de fibrocimento foi obtido do catálogo técnico da BRASILIT, referente ao modelo de telha ONDULADA de espessura seis milímetros.

As telhas são representadas por um carregamento distribuído no plano da cobertura, aplicado na direção vertical e distribuído nas barras que representam as terças. 
Na Figura 3.5, o plano da cobertura quadriculado mostra a área de aplicação da carga e a indicação -18 X nos diz o valor (18 daN/m²) entrando no plano de aplicação na direção vertical.

Os modelos Pratt C50 também foram analisados com o carregamento de telhas metálicas $\left(50 \mathrm{~N} / \mathrm{m}^{2}\right)$ e telhas cerâmicas $\left(800 \mathrm{~N} / \mathrm{m}^{2}\right)$.

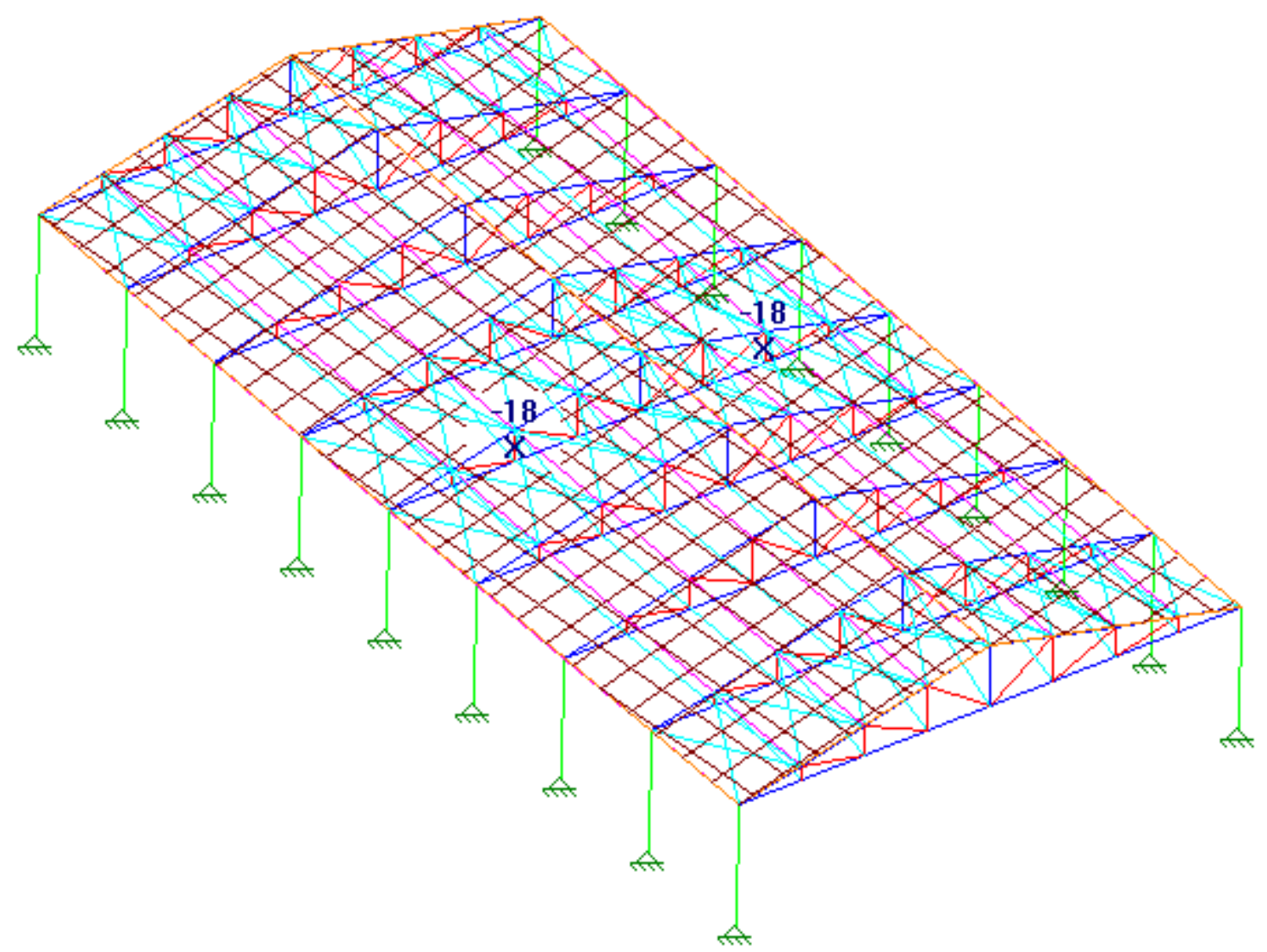

Figura 3.5 - Carregamento de telhas fibrocimento

\subsubsection{Ventos}

As ações devido a ventos foram estimadas conforme as prescrições da NBR 6123 (1988) Forças devidas ao vento em edificações. A intensidade das ações é calculada a partir da velocidade característica do vento e dos fatores: topográfico, de rugosidade e estatístico da edificação para determinação dos valores médios dos coeficientes de pressão. Os modelos considerados são referentes a um galpão na região industrial da cidade de São Carlos/SP, situado em terreno plano e inclinação da cobertura de 15 graus. As dimensões variam a cada 
modelo, sendo em planta: 12 x 24, 12 x 48, 18 x 24, 18 x 48, 24 x 48 e 24 x 96 metros, e alturas 3, 4 e 6 metros.

Foram adotadas características referentes à cidade de São Carlos por apresentar um valor de velocidade básica do vento intermediário - comparado aos apresentados pela NBR 6123 (1988) - e acima dos valores da maior parte do país, o que fornece uma margem de segurança para adoção de valores mínimos das ações.

\subsubsection{Ação estática de vento}

A seguir é mostrada a determinação da ação estática do vento q.

- Velocidade básica do vento (região de São Carlos):

$$
\mathrm{V}_{0}=40 \mathrm{~m} / \mathrm{s} \text {. }
$$

- Velocidade característica do vento:

$$
V_{k}=S_{1} \cdot S_{2} \cdot S_{3} \cdot V_{0}
$$

- $\mathrm{S}_{1}$ : fator topográfico.

Terreno plano com poucas ondulações:

$$
\mathrm{S} 1=1,0
$$

- $\mathrm{S}_{2}$ : fator de rugosidade do terreno e dimensões da edificação.

Rugosidade:

Área industrial (Categoria IV).

Dimensões:

Maior dimensão de 20 a 50 metros (Classe B).

Maior dimensão acima de 50 metros (Classe C). 
Os valores de $S_{2}$ da Tabela 3.6 foram obtidos pela Expressão 3.2:

$$
S_{2}=b \cdot\left(\frac{z}{10}\right)^{p}
$$

Tabela 3.6 - Valores de S2 para os modelos considerados

\begin{tabular}{|c|c|c|c|c|}
\hline Classe & $\mathbf{b}$ & $\mathbf{p}$ & Altura z (m) & $\mathbf{S}_{\mathbf{2}}$ \\
\hline \multirow{3}{*}{$\mathbf{B}$} & \multirow{3}{*}{0,85} & \multirow{2}{*}{0,125} & 3 & 0,73 \\
\cline { 4 - 5 } & & & 4 & 0,76 \\
\cline { 4 - 5 } & & & 6 & 0,79 \\
\hline \multirow{2}{*}{$\mathbf{C}$} & 0,84 & \multirow{2}{*}{0,135} & 3 & 0,71 \\
\cline { 4 - 5 } & & & 4 & 0,74 \\
\cline { 4 - 5 } & & & 6 & 0,78 \\
\hline
\end{tabular}

- $\mathrm{S}_{3}$ : fator estatístico

Indústria com alto fator de ocupação:

$$
\mathrm{S}_{3}=1,0 \text {. }
$$

Tabela 3.7 - Valores de Vk e ação estática q

\begin{tabular}{|c|c|c|c|c|c|}
\hline Classe & Altura $\mathbf{z}(\mathbf{m})$ & $\mathbf{S}_{\mathbf{1}} \mathbf{S}_{\mathbf{2}} \mathbf{.} \mathbf{S}_{\mathbf{3}}$ & $\mathbf{V}_{\mathbf{k}} \mathbf{( m / \mathbf { s } )}$ & $\mathbf{q}\left(\mathbf{N} / \mathbf{m}^{\mathbf{2}}\right)$ & Galpões \\
\hline \multirow{3}{*}{$\mathbf{B}$} & 3 & 0,73 & 29,2 & 533 & $1,4,7,10,14$ \\
\cline { 2 - 6 } & 4 & 0,76 & 30,4 & 578 & $2,5,8,11,15$ \\
\cline { 2 - 6 } & 6 & 0,79 & 31,6 & 624 & $3,6,9,12,13,16,17$ \\
\hline \multirow{3}{*}{$\mathbf{C}$} & 3 & 0,71 & 28,4 & 504 & 18 \\
\cline { 2 - 6 } & 4 & 0,74 & 29,6 & 548 & 19 \\
\cline { 2 - 6 } & 6 & 0,78 & 31,2 & 608 & 20 e 21 \\
\hline
\end{tabular}

As ações externas ou internas atuantes são resultado da multiplicação da ação estática pelo referente coeficiente de pressão. A Tabela 3.8 lista estes valores. 
Tabela 3.8 - Ação estática do vento multiplicada pelos coeficientes de pressão

\begin{tabular}{|c|c|c|c|c|c|c|}
\hline \multicolumn{7}{|c|}{ AÇÃO ATUANTE POR ÁREA } \\
\hline \multirow{2}{*}{$\begin{array}{c}\text { Ce ou } \\
\text { Ci }\end{array}$} & \multicolumn{5}{|c|}{ Ação estática do vento (daN/m²) } \\
\cline { 2 - 7 } & $\mathbf{5 3 , 3}$ & $\mathbf{6 2 , 4}$ & $\mathbf{5 0 , 4}$ & $\mathbf{6 0 , 8}$ & $\mathbf{5 7 , 8}$ & $\mathbf{5 4 , 8}$ \\
\hline $\mathbf{0 , 2}$ & 11 & 13 & 10 & 12 & 12 & 11 \\
\hline $\mathbf{0 , 3}$ & 16 & 19 & 15 & 18 & 17 & 16 \\
\hline $\mathbf{0 , 4}$ & 21 & 25 & 20 & 24 & 23 & 22 \\
\hline $\mathbf{0 , 5}$ & 27 & 31 & 25 & 30 & 29 & 27 \\
\hline $\mathbf{0 , 6}$ & 32 & 37 & 30 & 37 & 35 & 33 \\
\hline $\mathbf{0 , 7}$ & 37 & 44 & 35 & 43 & 41 & 38 \\
\hline $\mathbf{0 , 8}$ & 43 & 50 & 40 & 49 & 46 & 44 \\
\hline $\mathbf{0 , 9}$ & 48 & 56 & 45 & 55 & 52 & 49 \\
\hline $\mathbf{1 , 0}$ & 53 & 62 & 50 & 61 & 58 & 55 \\
\hline $\mathbf{1 , 2}$ & 64 & 75 & 61 & 73 & 69 & 66 \\
\hline
\end{tabular}

\subsubsection{Coeficientes de pressão externa}

Para cada galpão foram determinados os coeficientes de pressão externa de acordo com suas dimensões. A Figura 3.6 apresenta a divisão de áreas onde são aplicados diferentes valores das ações estáticas dos ventos. Cada face é identificada por uma sigla e os valores dos coeficientes de pressão externa da face são expostos na Tabela 3.9. A Tabela 3.10 fornece os valores da ação estática atuante em cada face. Segue abaixo a descrição de cada sigla:

B: barlavento;

L1: lateral 1;

C1: cobertura 1;

S: sotavento;

d1: distância 1. 
O sinal positivo indica sobrepressão externa, força no sentido de fora para dentro. De forma antagônica, o sinal negativo indica sucção externa, força de dentro para fora. Como mostrado na Figura 3.9.
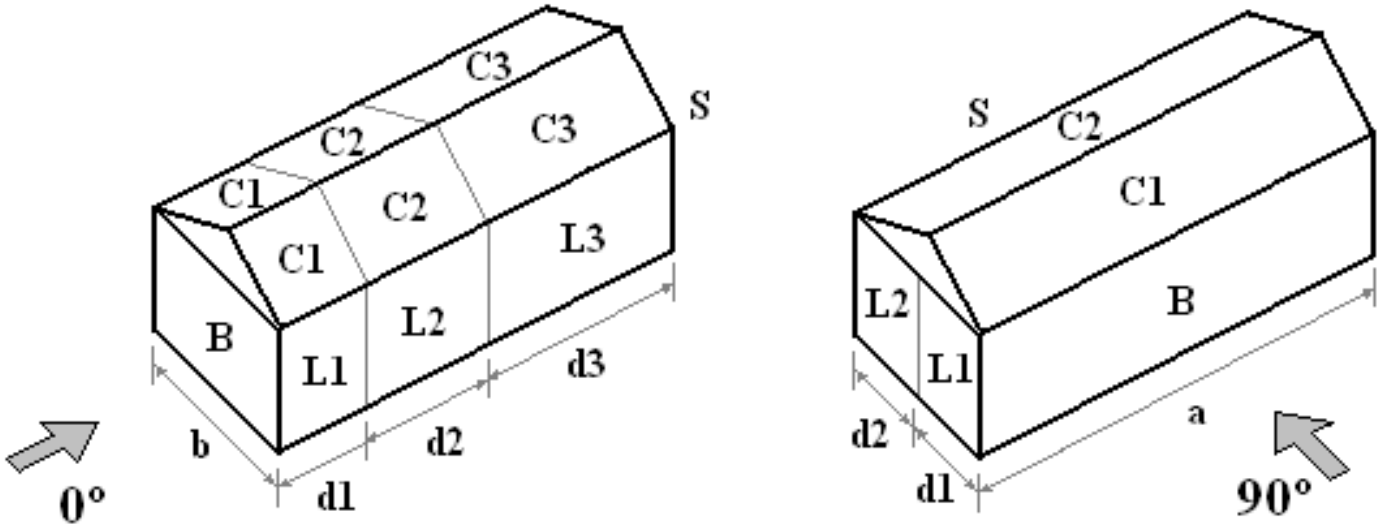

Figura 3.6 - Desenho esquemático de distâncias e faces de aplicação da ação estática dos ventos

Tabela 3.9 - Distâncias e coeficientes de pressão externa

\section{DISTÂNCIAS (m) E COEFICIENTES DE PRESSÃO EXTERNA}

\begin{tabular}{|c|c|c|c|c|c|c|c|c|c|c|c|c|c|c|c|c|c|c|c|c|}
\hline \multirow{2}{*}{\multicolumn{2}{|c|}{ FACE }} & \multicolumn{11}{|c|}{ VENTO A $0^{\circ}$} & \multicolumn{8}{|c|}{ VENTO A 90 } \\
\hline & & d1 & d2 & & $\mathbf{B}$ & C1 & $\mathrm{C} 2$ & $\mathrm{C} 3$ & L1 & 2 & 3 & $\mathrm{~S}$ & d1 & d2 & $\underline{\mathbf{D}}$ & C1 & $\mathrm{C} 2$ & L1 & $\mathrm{L} 2$ & 3 \\
\hline \multirow{21}{*}{ 量 } & 1 & 6 & 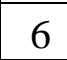 & 2 & 7 & $-0,8$ & $-0,6$ & $-0,2$ &, 8 &, 4 & & & & $c$ & 7 & $-1,0$ &, 4 & $-0,9$ & 0,5 & \\
\hline & 2 & 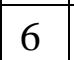 & & 2 & & $-0,8$ & $-0,6$ & $-0,2$ &, 8 & 4 & & & & & 7 & $-1,0$ & $-0,4$ & 9 &, 5 & \\
\hline & 3 & U & & & & $-0,8$ & $-0,6$ & & &, 4 & & & & & 7 & $-1,0$ & $-0,4$ & $-0,9$ & $-0,5$ & \\
\hline & 4 & 0 & & & & 8 & $-0,6$ & & &, 4 & & & & & 7 & $-1,0$ & 0,4 & &, 5 & \\
\hline & 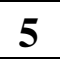 & 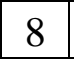 & & & & & $-0,6$ & & &, 4 & & & & & & & 4 & & & \\
\hline & 0 & 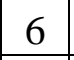 & & & & 8 & 6 & 2 & 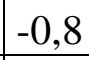 &, 4 & & & 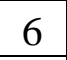 & 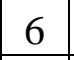 & 7 & $-1,0$ & 4 & 9 & 5 & - \\
\hline & 7 & 6 & & & & & 6 & & & & & & 6 & 1 & & & & & & \\
\hline & 8 & - & 6 & & 7 & $-0,8$ & $-0,6$ & 3 & 8 &, 5 & 3 & & 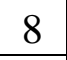 & 0 & 0,7 & $-1,0$ & $-0,4$ & 8 & $-0,4$ & 4 \\
\hline & 9 & 6 & 6 & 1 & 7 & $-0,8$ & $-0,6$ & $-0,3$ & $-0,8$ & 0,5 & $-0,3$ & $-0,4$ & 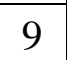 & 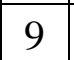 & 0,7 & $-1,0$ & $-0,4$ & $-0,8$ & $-0,4$ & $-0,4$ \\
\hline & 10 & 6 & 18 & & 7 & $-0,8$ & $-0,6$ & $-0,2$ & $-0,8$ & $-0,4$ & $-0,2$ & $-0,3$ & 6 & 2 & 0,7 & $-1,0$ & $-0,4$ & $-0,9$ & $-0,5$ & 0,5 \\
\hline & 11 & 8 & & & & $-0,8$ & $-0,6$ & $-0,2$ & ),8 & 0,4 & $-0,2$ & $-0,3$ & 8 & 0 & 0,7 & $-1,0$ & $-0,4$ & $-0,9$ & $-0,5$ & $-0,5$ \\
\hline & 12 & 2 & & & 7 & $-0,8$ & $-0,6$ & $-0,2$ & ,8 & 0,4 & & $-0,3$ & J & 9 & 0,7 & $-1,0$ & 4 & 9 & 5 & 0,5 \\
\hline & 13 & -1 & & & & 3 & & & & & & & & 9 & 7 & $-1,0$ &, 4 & & 5 & ,5 \\
\hline & & & & & & 3 & & & & & & & & 0 & & $-1,0$ &, 4 & & 5 & 0,5 \\
\hline & 15 & & & & & $-0,8$ & $-0,6$ & & & $-0,4$ & & & 8 & & 0,7 & $-1,0$ & $-0,4$ & & & $-0,5$ \\
\hline & 16 & & & & & $-0,8$ & $-0,6$ & & & $-0,4$ & & & & & 0,7 & $-1,0$ & $-0,4$ & & $-0,5$ & $-0,5$ \\
\hline & 17 & & & & & $-0,8$ & $-0,6$ & & & $-0,4$ & & $-0,3$ & & 2 & 0,7 & $-1,0$ & $-0,4$ & $-0,9$ & $-0,5$ & $-0,5$ \\
\hline & & 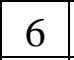 & & & & $-0,8$ & $-0,6$ & $-0,2$ & $-0,8$ & $-0,4$ & $-0,2$ & $-0,3$ & 8 & 4 & 0,7 & $-1,0$ & $-0,4$ & 9 & $-0,5$ & $-0,5$ \\
\hline & 19 & 0 & & & & $-0,8$ & $-0,6$ & & & & & & 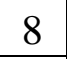 & 4 & 0,7 & $-1,0$ & $-0,4$ & & & $-0,5$ \\
\hline & 20 & & & & & & & & & & & & & 12 & 0,7 & $-1,0$ & $-0,4$ & & $-0,5$ & $-0,5$ \\
\hline & 21 & 12 & 36 & 48 & 0,7 & $-0,8$ & - 0 & ? & & $-0,4$ & & $-0,3$ & 12 & 12 & 0,7 & $-1,0$ & $-0,4$ & O 0 & $-0,5$ & 0 \\
\hline
\end{tabular}



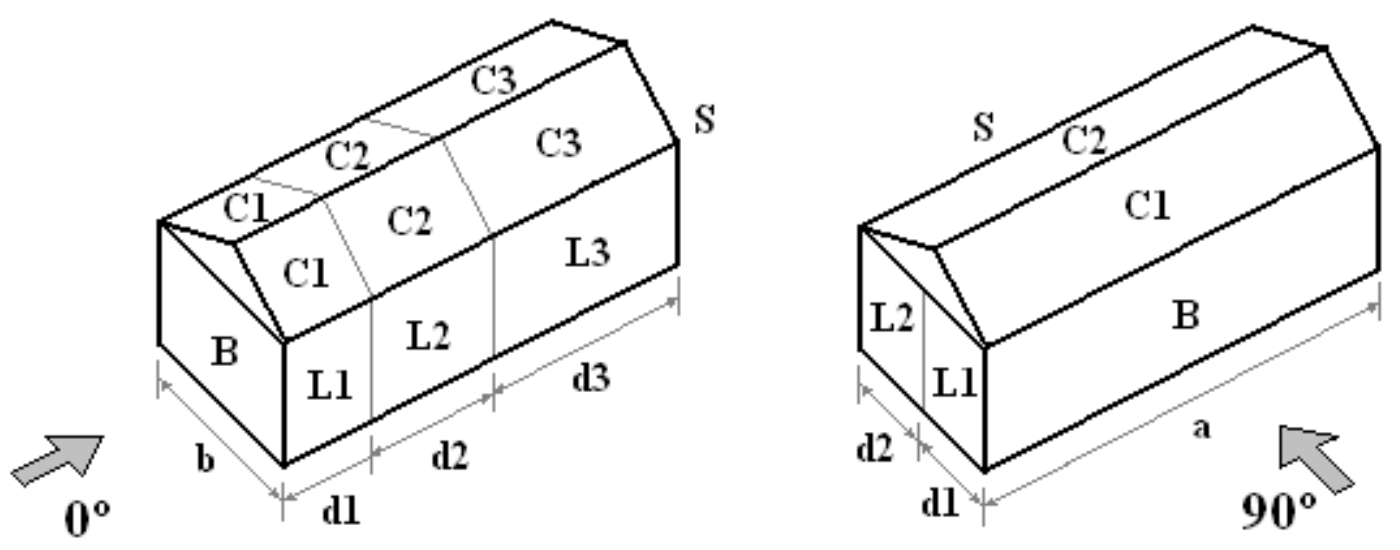

Figura 3.7 - Desenho esquemático de distâncias e faces de aplicação da ação estática dos ventos

Tabela 3.10 - Ação estática dos ventos em cada face

\begin{tabular}{|c|c|c|c|c|c|c|c|c|c|c|c|c|c|c|c|c|c|c|c|c|}
\hline & & & & & & & & & & & & & & & & & & & & \\
\hline & & & & & & & 19 & & & & & & & & & ENT & $U \mathrm{~A}$ & 5 & & \\
\hline & & d1 & d2 & d3 & B & C1 & $\mathrm{C} 2$ & C3 & L1 & L2 & L3 & $\mathbf{S}$ & d1 & d2 & B & C1 & C2 & L1 & L2 & S \\
\hline & 1 & $r$ & 6 & 12 & 37 & -43 & -32 & -11 & -43 & -21 & -11 & -16 & 6 & 6 & 37 & -53 & -21 & -48 & -27 & -27 \\
\hline & 2 & 6 & 6 & 12 & 0 & -46 & -35 & -12 & 46 & -23 & -12 & -17 & 6 & 6 & 40 & -58 & -23 & -52 & -29 & -29 \\
\hline & 3 & 6 & 6 & 12 & 1 & -50 & 7 & -12 & 0 & -25 & -12 & -19 & 6 & 6 & 44 & -62 & -25 & -56 & -31 & -31 \\
\hline & 4 & 6 & 18 & 24 & 7 & -43 & 2 & -11 & 3 & -21 & -11 & -16 & 6 & 6 & 37 & -53 & 21 & -48 & -27 & -27 \\
\hline & 5 & 8 & 16 & 24 & 0 & -46 & 5 & -12 & 6 & -23 & -12 & -17 & 6 & 6 & 40 & -58 & -23 & -52 & -29 & -29 \\
\hline & 6 & 6 & 10 & 24 & 4 & -50 & 7 & 12 & 0 & -25 & -12 & -19 & 6 & 6 & 44 & -62 & -25 & -56 & -31 & -31 \\
\hline & 7 & 6 & 6 & 12 & 7 & -43 & 2 & -16 & 3 & -27 & -16 & -21 & 6 & 12 & 37 & -53 & -21 & -43 & -21 & -21 \\
\hline & 8 & 6 & 6 & 12 & 0 & -46 & 5 & -17 & 6 & -29 & -17 & -23 & 8 & 10 & 40 & -58 & -23 & -46 & -23 & -23 \\
\hline & 9 & 6 & 6 & 12 & 44 & -50 & -37 & -19 & -50 & -31 & -19 & -25 & 9 & 9 & 44 & -62 & -25 & -50 & -25 & -25 \\
\hline & 10 & 6 & 18 & 7 & 37 & -43 & -32 & -11 & -43 & -21 & -11 & -16 & 6 & 12 & 37 & -53 & -21 & -48 & -27 & -27 \\
\hline & 11 & 8 & 16 & 24 & 40 & -46 & -35 & -12 & -46 & -23 & -12 & -17 & 8 & 10 & 40 & -58 & -23 & -52 & -29 & -29 \\
\hline & 12 & 12 & 12 & 24 & 44 & -50 & -37 & -12 & -50 & -25 & -12 & -19 & 9 & 9 & 44 & -62 & -25 & -56 & -31 & -31 \\
\hline & 13 & 12 & 12 & 24 & 44 & -50 & -37 & -12 & 0 & -25 & -12 & -19 & 9 & 9 & 44 & -62 & -25 & -56 & -31 & -31 \\
\hline & 14 & 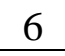 & 18 & 24 & 37 & -43 & -32 & -11 & 3 & -21 & -11 & -16 & 6 & 18 & 37 & -53 & -21 & -48 & -27 & -27 \\
\hline & 15 & 8 & 16 & 24 & 40 & -46 & 5 & -12 & 6 & -23 & -12 & -17 & 8 & 16 & 40 & -58 & -23 & -52 & -29 & -29 \\
\hline & 16 & 12 & 12 & 24 & 44 & -50 & -37 & -12 & 0 & -25 & -12 & -19 & 12 & 12 & 44 & -62 & -25 & -56 & -31 & -31 \\
\hline & 17 & 12 & 12 & 4 & 44 & -50 & -37 & -12 & 0 & -25 & -12 & -19 & 12 & 12 & 44 & -62 & -25 & -56 & -31 & -31 \\
\hline & 18 & 6 & 42 & 48 & 35 & -40 & -30 & -10 & 10 & -20 & -10 & -15 & 8 & 16 & 35 & -50 & -20 & -45 & -25 & -25 \\
\hline & 19 & 8 & 40 & 48 & 38 & -44 & -33 & -11 & 14 & -22 & -11 & -16 & 8 & 16 & 38 & -55 & -22 & -49 & -27 & -27 \\
\hline & 20 & 12 & 36 & 48 & 43 & -49 & -36 & -12 & -49 & -24 & -12 & -18 & 12 & 12 & 43 & -61 & -24 & -55 & -30 & -30 \\
\hline & 21 & 12 & 36 & 48 & 43 & -49 & -36 & -12 & -49 & -24 & -12 & -18 & 12 & 12 & 43 & -61 & -24 & -55 & -30 & -30 \\
\hline
\end{tabular}


No plano do telhado é aplicado um carregamento distribuído perpendicular á área, atuantes nas barras que representam as terças. No plano da face lateral do galpão é aplicado um carregamento distribuído perpendicular à área, atuante nos pilares.

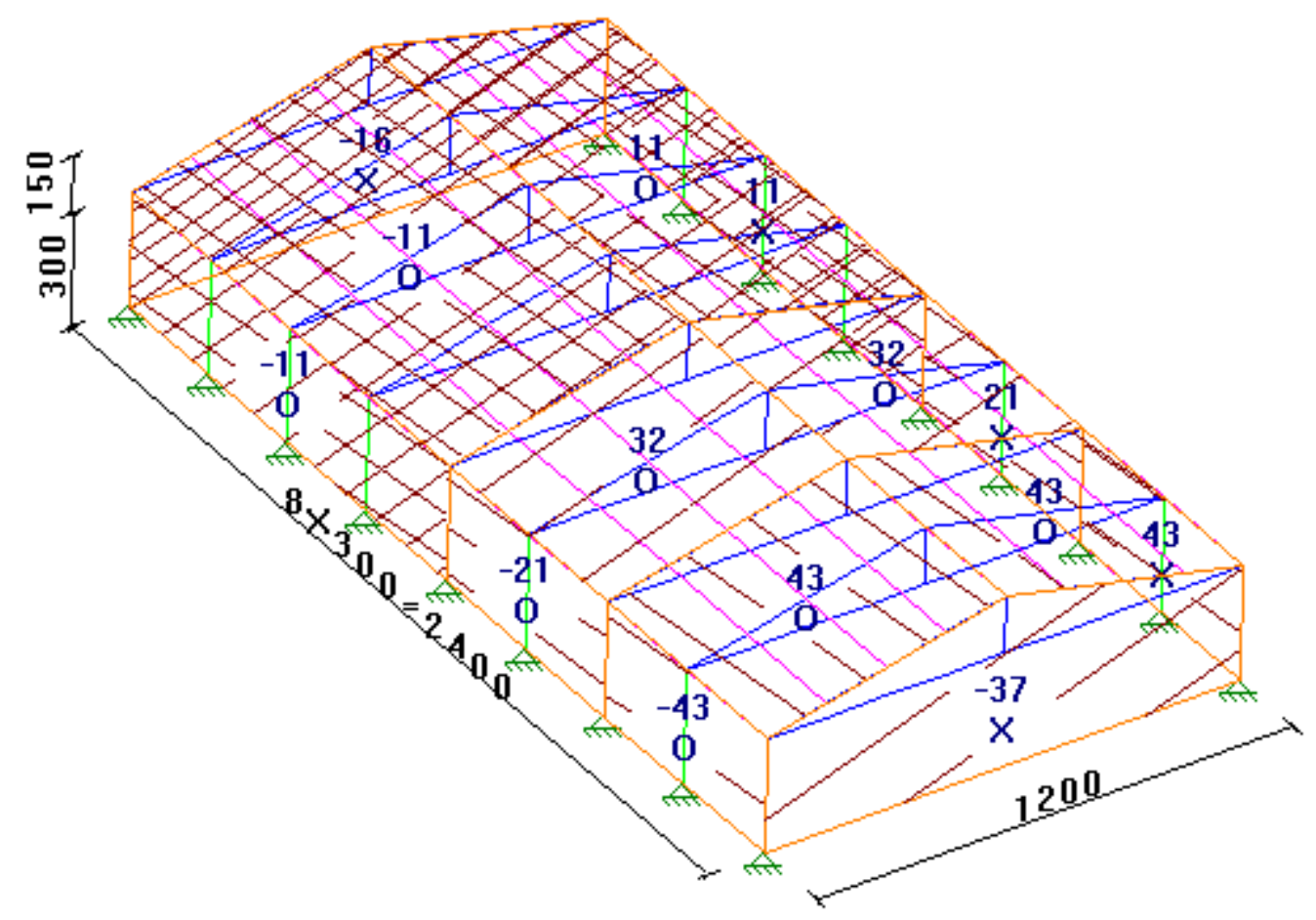

Figura 3.8 - Carregamento de sobrepressão do vento na direção longitudinal (vento a $0^{\circ}$ )

\subsubsection{Hipóteses de disposição das paredes de vedação}

O galpão, em uso, pode apresentar várias configurações de vedações e esta variação influencia na intensidade da ação do vento. A fim de avaliar as condições mais desfavoráveis ao contraventamento da cobertura, determinaram-se os quatro tipos de configuração descritos a seguir:

- completamente vedado;

- abertura a barlavento;

- abertura a sotavento;

- cobertura isolada (sem vedação). 


\subsubsection{Coeficientes de pressão interna}

Os valores, considerados na análise, são valores extremos que representam os casos mais desfavoráveis e apresentam maiores pressões de vento. São eles:

$\mathrm{C}_{\mathrm{pi}}=+0,8$ para sobrepressão interna com abertura dominante a barlavento e relação $A_{a d} / A_{a s}$ igual a 6;

$\mathrm{C}_{\mathrm{pi}}=-0,9$ sucção interna com abertura situada em zona de alta sucção externa e relação $A_{a d} / A_{\text {ase }}$ maior ou igual a 3.

Onde:

$A_{a d}=$ área da abertura dominante;

$A_{a s}=$ área total das aberturas succionadas nas outras faces;

$A_{a s e}=$ demais áreas de aberturas succionadas externamente.

Os sinais dos coeficientes de pressão interna são contrários aos de pressão externa, pois o sinal positivo indica sobrepressão interna, força no sentido de dentro para fora, e o sinal negativo indica sucção interna, força no sentido de fora para dentro.

Tabela 3.11 - Ação interna dos ventos

\begin{tabular}{|c|c|c|c|c|c|c|c|}
\hline \multirow{2}{*}{ Ci } & \multicolumn{6}{|c|}{ Ação característica do vento (daN/m²) } \\
\cline { 3 - 8 } \multicolumn{2}{|c|}{} & 53,3 & 62,4 & 50,4 & 60,8 & 57,8 & 54,8 \\
\hline Sobrepressão interna & $+0,8$ & 43 & 50 & 40 & 49 & 46 & 44 \\
\hline Sucção interna & $-0,9$ & 48 & 56 & 45 & 55 & 52 & 49 \\
\hline
\end{tabular}




\section{Pressão externa}
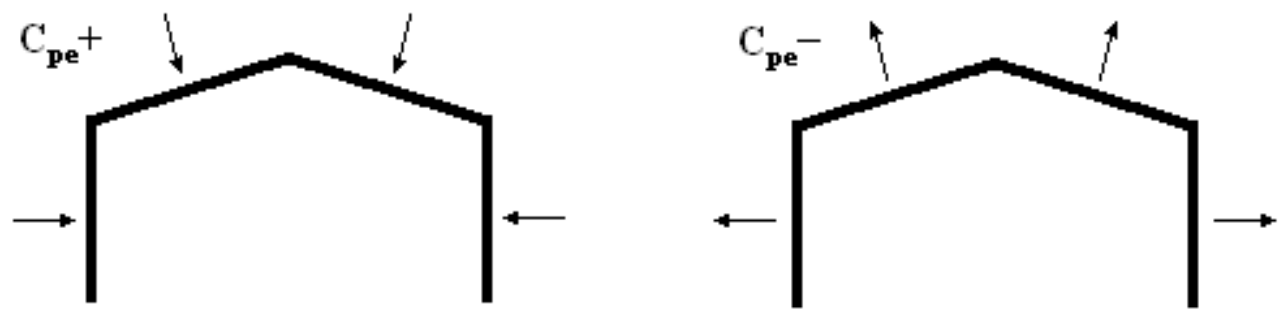

Pressão interna
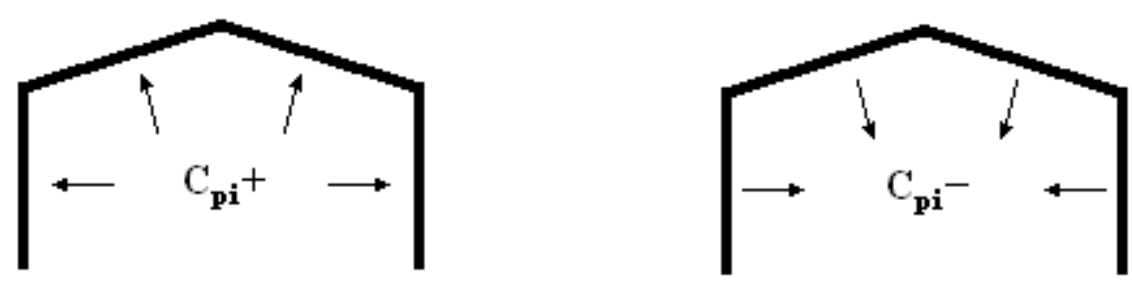

Figura 3.9 - Sentidos dos sinais dos coeficientes de pressão

\subsubsection{Coeficientes de pressão para coberturas isoladas}

A hipótese de cobertura isolada segue um procedimento diferente, do descrito nos itens 6.1 e 6.2 da NBR 6123 (1988), para determinação das ações dos ventos. Para as coberturas isoladas de duas águas a norma apresenta, no item 8.2, a formulação da Tabela 3.12.

Tabela 3.12 - Formulação para obtenção dos coeficientes de pressão para coberturas isoladas

\begin{tabular}{|c|c|c|}
\hline \multirow{3}{*}{ Coeficientes } & \multicolumn{2}{|c|}{$0,07 \leq \operatorname{tg} \theta \leq 0,4$} \\
\cline { 2 - 3 } & $1^{\circ}$ Carregamento & $2^{\circ}$ Carregamento \\
\hline $\mathrm{C}_{\mathrm{pb}}$ & $2,4 . \operatorname{tg} \theta+0,6$ & $0,6 . \operatorname{tg} \theta-0,74$ \\
\hline $\mathrm{C}_{\mathrm{ps}}$ & $3,0 . \operatorname{tg} \theta-0,5$ & $-1,0$ \\
\hline $\mathrm{Cpb}=$ coeficiente de pressão a barlavento $\quad \mathrm{C}_{\mathrm{bs}}=$ coeficiente de pressão a sotavento \\
\hline
\end{tabular}


Para os modelos considerados, com inclinação de $15^{\circ}$, tem-se os valores da Tabela 3.13.

Tabela 3.13 - Coeficientes de pressão para coberturas isoladas

\begin{tabular}{|c|c|c|}
\hline Coeficientes & \multicolumn{2}{|c|}{$0,07 \leq \operatorname{tg} 15^{\circ} \leq 0,4$} \\
\cline { 2 - 3 } & $1^{\circ}$ Carregamento & $2^{\circ}$ Carregamento \\
\hline $\mathrm{C}_{\mathrm{pb}}$ & $+1,2$ & $-0,6$ \\
\hline $\mathrm{C}_{\mathrm{ps}}$ & $+0,3$ & $-1,0$ \\
\hline
\end{tabular}

Tabela 3.14 - Ação dos ventos em coberturas isoladas

\begin{tabular}{|c|c|c|c|c|c|c|c|}
\hline \multirow{2}{*}{ Ce } & \multicolumn{5}{|c|}{ Ação característica do vento (daN/m²) } \\
\cline { 3 - 8 } \multicolumn{2}{|c|}{} & 53,3 & 62,4 & 50,4 & 60,8 & 57,8 & 54,8 \\
\hline \multirow{2}{*}{$1^{\circ}$ carregamento } & 1,2 & 64 & 75 & 61 & 73 & 69 & 66 \\
\cline { 2 - 8 } & 0,3 & 16 & 19 & 15 & 18 & 17 & 16 \\
\hline \multirow{2}{*}{$2^{\circ}$ carregamento } & 0,6 & 32 & 37 & 30 & 37 & 35 & 33 \\
\cline { 2 - 8 } & 1,0 & 53 & 62 & 50 & 61 & 58 & 55 \\
\hline
\end{tabular}

\subsubsection{Carregamentos e combinações}

Para analisar todas as hipóteses de atuação das ações, foram criados oito carregamentos e nove combinações. As combinações representam as possíveis formas de atuação de vento, a primeira sendo em sua ausência e as demais alternando as diferentes hipóteses de vedação do galpão.

As combinações seguem os procedimentos do item 5.7 Combinações de ações em estados limites últimos da NBR 7190 (1997) para combinações últimas normais. Como é considerada apenas uma ação variável, o vento, todos os valores das ações são multiplicados por 1,4 adotando a Expressão 3.3: 


$$
F_{d}=\sum \gamma_{G i} \cdot F_{G i, k}+\gamma_{Q} \cdot\left[F_{Q 1, k}+\sum \psi_{0 j} \cdot F_{Q j, k}\right]
$$

Onde:

$$
\begin{aligned}
& F_{d}=\text { força de cálculo } \\
& \gamma_{G i}=\text { coeficiente majorador das ações permanentes } \\
& F_{G i, k}=\text { forças permanentes características } \\
& \gamma_{Q}=\text { coeficiente de majoração das ações variáveis } \\
& F_{Q 1, k}=\text { força variável principal característica } \\
& \psi_{0}=\text { coeficiente minorador de simultaneidade de ações variáveis } \\
& F_{Q j, k}=\text { forças variáveis características }
\end{aligned}
$$

A seguir são listados os carregamentos e combinações.

\section{Carregamentos:}

Cg1 = Peso próprio

Cg2 = Telhas

Cg3 = Vento a $0^{\circ}$ externo

Cg4 = Vento a $90^{\circ}$ externo

Cg5 = Vento sobrepressão interna

Cg6 = Vento sucção interna

Cg7 = Vento sobrepressão em cobertura isolada

Cg8 = Vento sucção em cobertura isolada 


\section{Combinações:}

$$
\begin{aligned}
& \text { Comb1 }=1,4 \times(\mathrm{Cg} 1+\mathrm{Cg} 2) \\
& \text { Comb2 }=1,4 \times(\mathrm{Cg} 1+\mathrm{Cg} 2+\mathrm{Cg} 3) \\
& \text { Comb3 }=1,4 \times(\mathrm{Cg} 1+\mathrm{Cg} 2+\mathrm{Cg} 4) \\
& \text { Comb4 }=1,4 \times(\mathrm{Cg} 1+\mathrm{Cg} 2+\mathrm{Cg} 3+\mathrm{Cg} 5) \\
& \text { Comb5 }=1,4 \times(\mathrm{Cg} 1+\mathrm{Cg} 2+\mathrm{Cg} 3+\mathrm{Cg} 6) \\
& \text { Comb6 }=1,4 \times(\mathrm{Cg} 1+\mathrm{Cg} 2+\mathrm{Cg} 4+\mathrm{Cg} 5) \\
& \text { Comb7 }=1,4 \times(\mathrm{Cg} 1+\mathrm{Cg} 2+\mathrm{Cg} 4+\mathrm{Cg} 6) \\
& \text { Comb8 }=1,4 \times(\mathrm{Cg} 1+\mathrm{Cg} 2+\mathrm{Cg} 7) \\
& \text { Comb9 }=1,4 \times(\mathrm{Cg} 1+\mathrm{Cg} 2+\mathrm{Cg} 8)
\end{aligned}
$$

\subsection{Disposição do contraventamento}

O contraventamento segue a recomendação da NBR 7190 (1997) de um para cada três vãos entre treliças. Nos modelos foi adotada a configuração com menor número de vãos contraventados e, sempre que possível, simetricamente para cada metade ao longo do comprimento do galpão.

Ao longo das treliças os nós contraventados foram dispostos de duas formas para cada modelo tridimensional:

- todos os nós contraventados

- um a cada dois nós contraventado 
A Figura 3.2 apresenta um exemplo de contraventamento disposto de forma simétrica ao longo do comprimento, em um a cada três vãos entre treliças, e com um a cada dois nós da treliça contraventado. A Figura 3.5 mostra um modelo com todos os nós contraventados. 


\section{APRESENTAÇÃO DOS RESULTADOS}

Este capítulo descreve inicialmente os métodos de obtenção dos resultados dos modelos numéricos computacionais. Em seguida são apresentados os resultados obtidos pelos métodos simplificados e pela modelagem numérica. Os métodos simplificados são os métodos que se utilizam apenas da análise plana da treliça e obtém as forças nos contraventamentos a partir da força axial máxima no banzo comprimido e do número de treliças contraventadas.

Os resultados analisados nos modelos numéricos foram:

- deslocamentos: para determinação das seções das barras utilizadas;

- forças axiais nas treliças;

- forças axiais nas barras de contraventamento.

\subsection{Obtenção dos resultados}

\subsubsection{Métodos simplificados}

Os métodos simplificados são os propostos pelos documentos normativos e por UNDERWOOD (2000), como estudados no Capítulo 2. Foram adotados quatro métodos, a seguir são mostrados os métodos e a correspondente formulação adotada:

1) NBR 7190 (1997):

$$
F_{1 d}=\frac{N_{d}}{150} \quad \text { e } \quad F_{d}=\frac{2}{3} n \cdot F_{1 d}
$$

Onde:

$$
\begin{aligned}
& N_{d}=\text { força máxima na barra contraventada } \\
& n=\text { número de treliças contraventadas }
\end{aligned}
$$

2) EUROCODE 5:

$$
F_{1 d}=\frac{N_{d}}{50} \quad \text { e } \quad F_{d}=n \cdot F_{1 d}
$$


3) UNDERWOOD (2000):

$$
F_{1 d}=\frac{N_{d}}{32} \quad \text { e } \quad F_{d}=n \cdot F_{1 d}
$$

4) SABS 0163:

$$
P_{L}=\frac{0,10 \cdot P_{A}}{(N+1)} \quad \text { e } \quad C_{P L N}=P_{L} \cdot n^{0,7}
$$

Onde:

$$
\begin{aligned}
& P_{L}=\text { força em cada elemento de contraventamento lateral } \\
& P_{A}=\text { força axial máxima na treliça devido à carga permanente } \\
& N=\text { número de restrições ao longo da treliça } \\
& n=\text { número de treliças contraventadas }
\end{aligned}
$$

O valor de $N$ no método SABS foi adotado para o caso de menor número de restrições laterais, no caso de um a cada dois nós contraventado. Sendo, assim, $N=3$ para as treliças de 12 metros, $N=5$ para as treliças de 18 metros e $N=7$ para as treliças de 24 metros.

\subsubsection{Modelos numéricos tridimensionais}

Os deslocamentos foram avaliados por uma combinação ELS - Estado Limite de Serviço - que aplica os carregamentos do peso próprio e das telhas. A Figura 4.1 e a Figura 4.2 mostram os deslocamentos e as posições deformadas da tesoura e do galpão de doze metros de vão e telhas de fibrocimento, obtidos no STRAP.

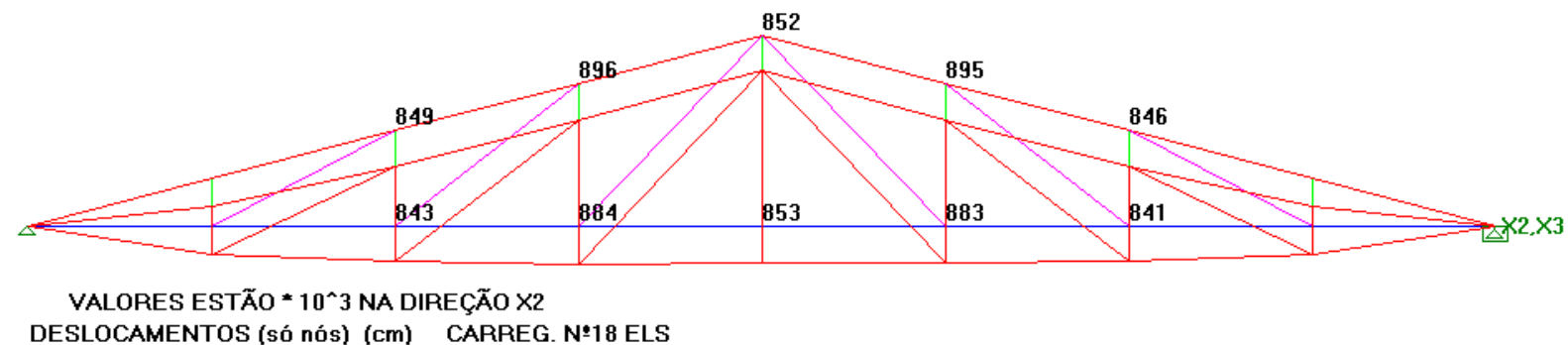

Figura 4.1 - Deslocamentos e deformada da Treliça 01 Pratt C50 (STRAP) 


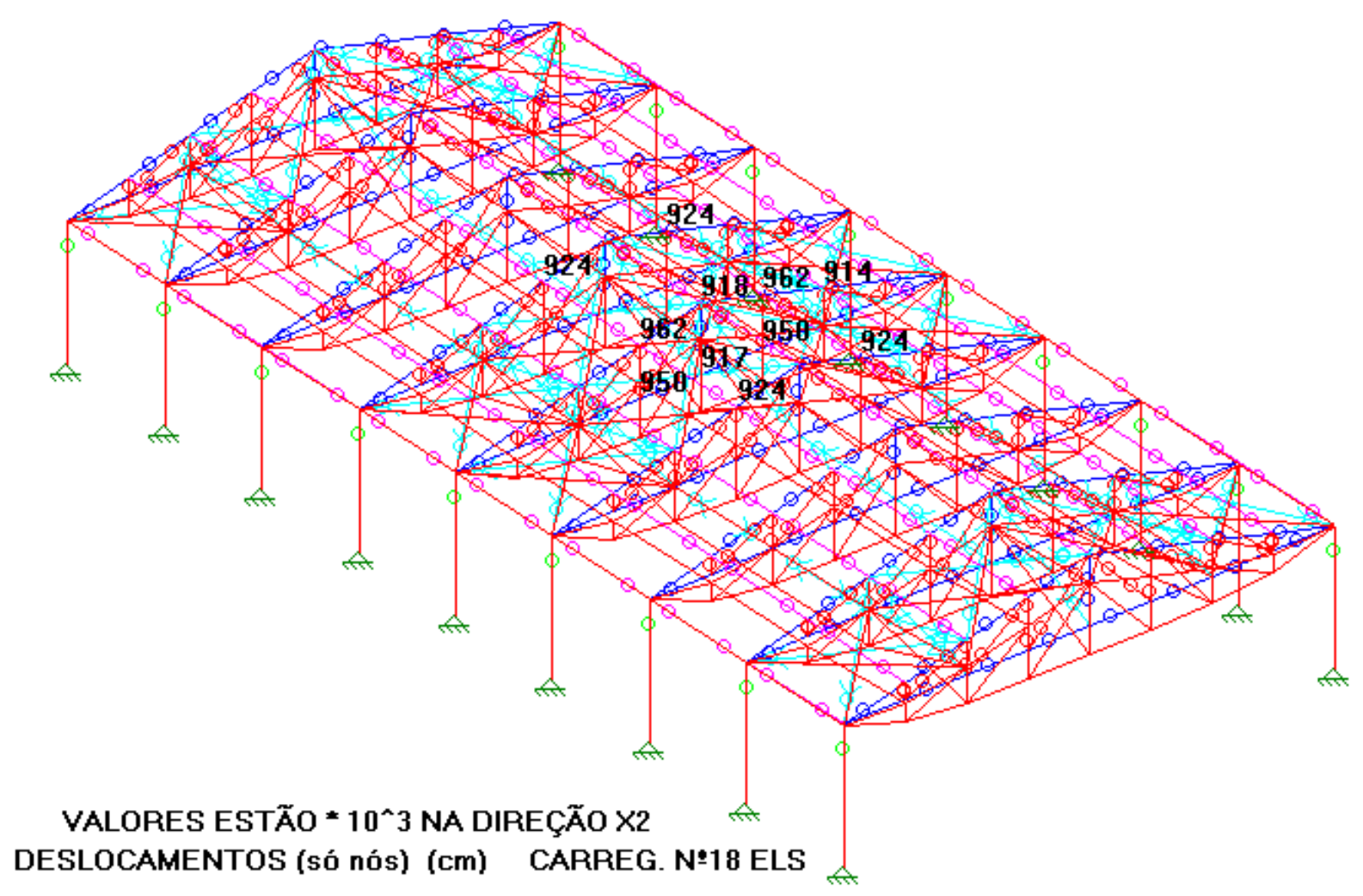

Figura 4.2 - Deslocamentos e deformada do Galpão 01 Pratt C50

As forças axiais nas barras são determinadas pela envoltória dos carregamentos, em que cada barra apresenta o valor máximo resultante das combinações determinadas. Os resultados podem ser exibidos das seguintes formas: escritos ao lado de cada barra; exibindo o diagrama de cada barra; como relatório especificando os valores máximos e mínimos e as respectivas combinações; como relatório com os diagramas de cada barra.

A Figura 4.3 mostra valores máximos e mínimos de forças axiais escritos nas barras de pilares, nas de extremidades dos banzos superiores e a tração máxima nas barras de contraventamento vertical (em azul claro). Os valores positivos são de compressão e os negativos de tração. 


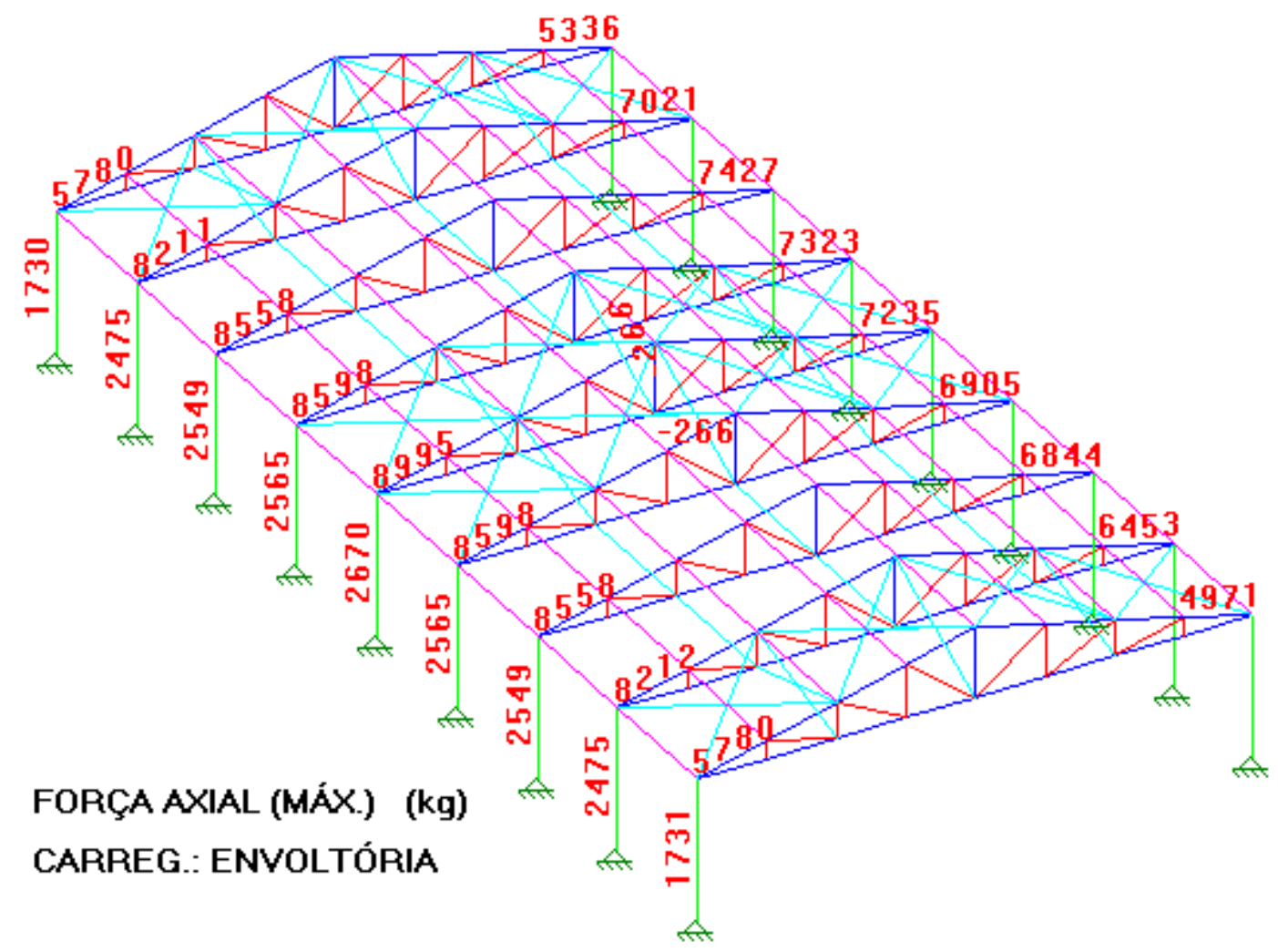

Figura 4.3 - Forças axiais máximas do Galpão 01

\subsection{Resultados das treliças planas}

As treliças são divididas de acordo com o tipo de treliça (Howe ou Pratt), a classe de madeira adotada (C30 ou C50), e o tipo de telha (fibrocimento, metálica ou cerâmica). 


\subsubsection{Treliças HOWE C50 telha fibrocimento}

Tabela 4.1 - Forças nos contraventamentos segundo os métodos simplificados (HOWE C50)

\begin{tabular}{|c|c|c|c|c|c|c|c|c|c|c|c|c|}
\hline & & & GE & OMEI & TRIAS & DAS T & TRELI & ÇAS & & & & \\
\hline $\bar{L}\left(\mathrm{v}_{\mathrm{a}}^{*}\right.$ & & 12 & 12 & 12 & 18 & 18 & 18 & 18 & 24 & 24 & 24 & 24 \\
\hline h (altura & ntral) & 1,5 & 1,5 & 1,5 & 2,25 & 2,25 & 2,25 & 2,25 & 3 & 3 & 3 & 3 \\
\hline $\mathrm{z}$ (altura $\mathrm{c}$ & pilar) & 3 & 4 & 6 & 3 & 4 & 6 & 6 & 3 & 4 & 6 & 6 \\
\hline e (dist. entr & reliças) & 3 & 3 & 3 & 3 & 3 & 3 & 4 & 3 & 3 & 3 & 4 \\
\hline Compressã & áx $(\mathrm{kN})$ & 82,4 & 85,8 & 92,0 & 156,1 & 161,3 & 155,3 & 197,8 & 232,5 & 240,0 & 249,5 & 300,0 \\
\hline & & & & MÉTO & DO N & BR 719 & $90(199$ & & & & & \\
\hline & $\mathrm{F}_{1 \mathrm{~d}}$ & 0,5 & 0,6 & 0,6 & 1,0 & 1,1 & 1,0 & 1,3 & 1,5 & 1,6 & 1,7 & 2,0 \\
\hline & \begin{tabular}{|ll}
$\mathrm{n}$ & 7
\end{tabular} & 2,6 & 2,7 & 2,9 & 4,9 & 5,0 & 4,8 & - & - & - & - & - \\
\hline$F_{3}(\mathbf{k N})$ & n 11 & - & - & - & - & - & - & 9,7 & - & - & - & 14,7 \\
\hline$T_{\mathrm{d}}(\mathrm{N})$ & n 15 & 5,5 & 5,7 & 6,1 & 10,4 & 10,8 & 10,4 & & 15,5 & 16,0 & 16,6 & - \\
\hline & n 23 & - & & - & & & & - & - & - & - & 30,7 \\
\hline & n 31 & - & - & - & - & - & - & - & 32,0 & 33,1 & 34,4 & - \\
\hline & & & & MÉTC & ODO E & UROC & CODE & & & & & \\
\hline & $\mathrm{F}_{1 \mathrm{~d}}$ & 1,6 & 1,7 & 1,8 & 3,1 & 3,2 & 3,1 & 4,0 & 4,6 & 4,8 & 5,0 & 6,0 \\
\hline & \begin{tabular}{|ll}
$\mathrm{n} \quad 7$ \\
\end{tabular} & 7,7 & 8,0 & 8,6 & 14,6 & 15,1 & 14,5 & - & - & - & - & - \\
\hline$F_{3}(\mathbf{k N})$ & n 11 & - & - & - & - & - & - & 29,0 & - & - & - & 44,0 \\
\hline$F_{d}(K I)$ & n 15 & 16,5 & 17,2 & 18,4 & 31,2 & 32,3 & 31,1 & & 46,5 & 48,0 & 49,9 & - \\
\hline & n 23 & - & & & & & & - & - & - & - & 92,0 \\
\hline & n 31 & - & & & & & & & 96,1 & 99,2 & 103,1 & - \\
\hline & & & MÉ & TODC & D UND & ERWC & OOD (2 & 2000) & & & & \\
\hline & $F_{1 d}$ & 2,6 & 2,7 & 2,9 & 4,9 & 5,0 & 4,9 & 6,2 & 7,3 & 7,5 & 7,8 & 9,4 \\
\hline & \begin{tabular}{|ll}
$\mathrm{n}$ & 7
\end{tabular} & 12,0 & 12,5 & 13,4 & 22,8 & 23,5 & 22,6 & - & - & - & - & - \\
\hline$E_{1}(\mathbf{l} \mathbf{N}$ & n 11 & - & & & & & & 45,3 & - & - & - & 68,7 \\
\hline$F_{d}(K N)$ & n 15 & 25,7 & 26,8 & 28,8 & 48,8 & 50,4 & 48,5 & & 72,7 & 75,0 & 78,0 & - \\
\hline & n 23 & - & & & & & & - & - & - & - & 143,7 \\
\hline & n 31 & - & - & - & - & - & - & - & 150,1 & 155,0 & 161,1 & - \\
\hline & & & & MÉ & TODO & SABS & 0163 & & & & & \\
\hline Compressãc & $\mathrm{rm}(\mathrm{kN})$ & & 39,3 & & & 77,0 & & 85,8 & & 134,3 & & 146,3 \\
\hline & $F_{1 d}$ & & 1,0 & & & 1,3 & & 1,4 & & 1,7 & & 1,8 \\
\hline & & 4,6 & 4,6 & 4,6 & 6,0 & 6,0 & 6,0 & - & - & - & - & - \\
\hline$F_{d}(\mathbf{k N})$ & n 11 & - & & & & & & 10,5 & - & - & - & 13,4 \\
\hline$F_{d}(\mathrm{KN})$ & n 15 & 9,8 & 12,8 & 9,8 & 12,8 & 12,8 & 12,8 & & 16,8 & 16,8 & 16,8 & \\
\hline & n 23 & - & & - & & & & $\begin{array}{lll}- & \text { - }\end{array}$ & - & - & - & 28,0 \\
\hline & n 31 & - & & & & & & & 34,7 & 34,7 & 34,7 & 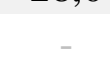 \\
\hline
\end{tabular}




\subsubsection{Treliças HOWE C30 telha fibrocimento}

Tabela 4.2 - Forças nos contraventamentos segundo os métodos simplificados (HOWE C30)

\begin{tabular}{|c|c|c|c|c|c|c|c|c|c|c|c|c|}
\hline & & & GE & OME & TRIAS & DAS I & TRELI & ÇAS & & & & \\
\hline L (vã & & 12 & 12 & 12 & 18 & 18 & 18 & 18 & 24 & 24 & 24 & 24 \\
\hline h (altura c & ntral) & 1,5 & 1,5 & 1,5 & 2,25 & 2,25 & 2,25 & 2,25 & 3 & 3 & 3 & 3 \\
\hline $\mathrm{z}$ (altura d & pilar) & 3 & 4 & 6 & 3 & 4 & 6 & 6 & 3 & 4 & 6 & 6 \\
\hline e (dist. entre & (reliças) & 3 & 3 & 3 & 3 & 3 & 3 & 4 & 3 & 3 & 3 & 4 \\
\hline Compressãc & רáx $(\mathrm{kN})$ & 83,0 & 86,4 & 90,8 & 155,6 & 161,1 & 168,0 & 207,1 & 237,0 & 244,5 & 254,0 & 304,4 \\
\hline & & & & MÉTC & DO N & BR 719 & 0 (199 & & & & & \\
\hline & $F_{1 d}$ & 0,6 & 0,6 & 0,6 & 1,0 & 1,1 & 1,1 & 1,4 & 1,6 & 1,6 & 1,7 & 2,0 \\
\hline & \begin{tabular}{|ll}
$\mathrm{n}$ & 7
\end{tabular} & 2,6 & 2,7 & 2,8 & 4,8 & 5,0 & 5,2 & - & - & - & - & - \\
\hline$F_{3}(\mathrm{kN})$ & n 11 & - & - & - & - & - & - & 10,1 & - & - & - & 14,9 \\
\hline$F_{d}(\mathbf{k} \mid \bar{v})$ & n 15 & 5,5 & 5,8 & 6,1 & 10,4 & 10,7 & 11,2 & & 15,8 & 16,3 & 16,9 & \\
\hline & n 23 & - & - & - & - & - & - & - & & & & 31,1 \\
\hline & n 31 & - & - & - & - & - & - & - & 32,7 & 33,7 & 35,0 & - \\
\hline & & & & MÉT & ODO F & UROC & CODE & & & & & \\
\hline & $F_{1 d}$ & 1,7 & 1,7 & 1,8 & 3,1 & 3,2 & 3,4 & 4,1 & 4,7 & 4,9 & 5,1 & 6,1 \\
\hline & \begin{tabular}{|ll}
$\mathrm{n}$ & 7
\end{tabular} & 7,7 & 8,1 & 8,5 & 14,5 & 15,0 & 15,7 & - & - & $\begin{array}{lll}- & \\
\end{array}$ & - & - \\
\hline$F_{3}(k N)$ & n 11 & - & - & - & - & - & - & 30,4 & - & - & - & 44,6 \\
\hline$F_{d}(K / V)$ & n 15 & 16,6 & 17,3 & 18,2 & 31,1 & 32,2 & 33,6 & & 47,4 & 48,9 & 50,8 & \\
\hline & n 23 & - & - & - & - & - & - & - & & & & 93,3 \\
\hline & n 31 & - & - & & - & & & & 98,0 & 101,1 & 105,0 & \\
\hline & & & MÉ & TOD & DUND & ERWC & DOD ( & 2000) & & & & \\
\hline & $F_{1 d}$ & 2,6 & 2,7 & 2,8 & 4,9 & 5,0 & 5,3 & 6,5 & 7,4 & 7,6 & 7,9 & 9,5 \\
\hline & \begin{tabular}{|ll}
$\mathrm{n}$ & 7
\end{tabular} & 12,1 & 12,6 & 13,2 & 22,7 & 23,5 & 24,5 & - & - & - & - & - \\
\hline$F_{d}(k N)$ & n 11 & - & - & & - & - & - & 47,5 & & & & 69,8 \\
\hline$F_{d}(K \mid V)$ & n 15 & 25,9 & 27,0 & 28,4 & 48,6 & 50,3 & 52,5 & & 74,1 & 76,4 & 79,4 & \\
\hline & n 23 & - & - & - & - & - & - & - & & & & 145,9 \\
\hline & n 31 & - & - & - & - & - & - & - & 153,1 & 157,9 & 164,0 & - \\
\hline & & & & MÉ & TODC & SABS & 0163 & & & & & \\
\hline Compressão & $\mathrm{erm}(\mathrm{kN})$ & & 38,1 & & & 84,1 & & 95,2 & & 138,8 & & 150,8 \\
\hline & $F_{1 d}$ & & 1,0 & & & 1,4 & & 1,6 & & 1,7 & & 1,9 \\
\hline & & 4,4 & 4,4 & 4,4 & 6,5 & 6,5 & 6,5 & - & - & - & - & - \\
\hline & n 11 & - & - & - & - & - & - & 11,6 & & & & 13,8 \\
\hline$F_{d}(k N)$ & n 15 & 9,5 & 14,0 & 9,5 & 14,0 & 14,0 & 14,0 & - & 17,4 & 17,4 & 17,4 & 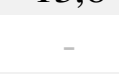 \\
\hline & n 23 & - & - & - & - & - & - & - & - & & & 28,9 \\
\hline & n 31 & - & & & & & & & 35,9 & 35,9 & 35,9 & \\
\hline
\end{tabular}




\subsubsection{Treliças PRATT C50 telha fibrocimento}

Tabela 4.3 - Forças nos contraventamentos segundo os métodos simplificados (PRATT C50)

\begin{tabular}{|c|c|c|c|c|c|c|c|c|c|c|c|c|}
\hline & & & GE & OMEI & TRIAS & DAS T & TRELI & ÇAS & & & & \\
\hline $\bar{L}\left(\mathrm{v}_{\mathrm{a}}^{*}\right.$ & & 12 & 12 & 12 & 18 & 18 & 18 & 18 & 24 & 24 & 24 & 24 \\
\hline h (altura & ntral) & 1,5 & 1,5 & 1,5 & 2,25 & 2,25 & 2,25 & 2,25 & 3 & 3 & 3 & 3 \\
\hline $\mathrm{z}$ (altura $\mathrm{c}$ & pilar) & 3 & 4 & 6 & 3 & 4 & 6 & 6 & 3 & 4 & 6 & 6 \\
\hline e (dist. entr & reliças) & 3 & 3 & 3 & 3 & 3 & 3 & 4 & 3 & 3 & 3 & 4 \\
\hline Compressã & áx $(\mathrm{kN})$ & 82,4 & 85,8 & 82,2 & 157,3 & 161,6 & 162,6 & 198,2 & 233,4 & 240,0 & 250,4 & 300,9 \\
\hline & & & & MÉTO & DO N & BR 719 & $0(199$ & & & & & \\
\hline & $\mathrm{F}_{1 \mathrm{~d}}$ & 0,5 & 0,6 & 0,5 & 1,0 & 1,1 & 1,1 & 1,3 & 1,6 & 1,6 & 1,7 & 2,0 \\
\hline & \begin{tabular}{|ll}
$\mathrm{n}$ & 7
\end{tabular} & 2,6 & 2,7 & 2,6 & 4,9 & 5,0 & 5,1 & - & - & - & - & - \\
\hline$F_{3}(\mathbf{k N})$ & n 11 & - & - & - & - & - & - & 9,7 & - & - & - & 14,7 \\
\hline$T_{\mathrm{d}}(\mathrm{N})$ & n 15 & 5,5 & 5,7 & 5,5 & 10,5 & 10,8 & 10,8 & & 15,6 & 16,0 & 16,7 & - \\
\hline & n 23 & - & & - & & & & - & - & - & - & 30,8 \\
\hline & n 31 & - & - & - & - & - & - & - & 32,2 & 33,1 & 34,5 & 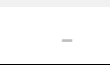 \\
\hline & & & & MÉTC & ODO E & UROC & CODE & & & & & \\
\hline & $\mathrm{F}_{1 \mathrm{~d}}$ & 1,6 & 1,7 & 1,6 & 3,1 & 3,2 & 3,3 & 4,0 & 4,7 & 4,8 & 5,0 & 6,0 \\
\hline & \begin{tabular}{|ll}
$\mathrm{n} \quad 7$ \\
\end{tabular} & 7,7 & 8,0 & 7,7 & 14,7 & 15,1 & 15,2 & - & - & - & - & - \\
\hline$F_{3}(\mathbf{k N})$ & n 11 & - & - & - & - & - & - & 29,1 & - & - & - & 44,1 \\
\hline$F_{d}(K I)$ & n 15 & 16,5 & 17,2 & 16,4 & 31,5 & 32,3 & 32,5 & & 46,7 & 48,0 & 50,1 & - \\
\hline & n 23 & - & & & & & & - & - & - & - & 92,3 \\
\hline & n 31 & - & & & & & & & 96,5 & 99,2 & 103,5 & - \\
\hline & & & MÉ & TODC & D UND & ERWC & OD (2 & 2000) & & & & \\
\hline & $F_{1 d}$ & 2,6 & 2,7 & 2,6 & 4,9 & 5,1 & 5,1 & 6,2 & 7,3 & 7,5 & 7,8 & 9,4 \\
\hline & \begin{tabular}{|ll}
$\mathrm{n}$ & 7
\end{tabular} & 12,0 & 12,5 & 12,0 & 22,9 & 23,6 & 23,7 & - & - & - & - & - \\
\hline$F_{d}(k N)$ & n 11 & - & & & & & & 45,4 & - & - & - & 69,0 \\
\hline$F_{d}(K N)$ & n 15 & 25,8 & 26,8 & 25,7 & 49,2 & 50,5 & 50,8 & & 72,9 & 75,0 & 78,3 & - \\
\hline & n 23 & - & & & & & & - & - & - & - & 144,2 \\
\hline & n 31 & - & - & - & - & - & - & - & 150,7 & 155,0 & 161,7 & - \\
\hline & & & & MÉ & TODO & SABS & 0163 & & & & & \\
\hline Compressãc & $\mathrm{rm}(\mathrm{kN})$ & & 39,5 & & & 77,4 & & 86,2 & & 135,2 & & 147,3 \\
\hline & $F_{1 d}$ & & 1,0 & & & 1,3 & & 1,4 & & 1,7 & & 1,8 \\
\hline & & 4,6 & 4,6 & 4,6 & 6,0 & 6,0 & 6,0 & - & - & $\begin{array}{lll}- & \\
\end{array}$ & - & - \\
\hline$F_{d}(\mathbf{k N})$ & n 11 & - & & & & & & 10,5 & - & - & - & 13,5 \\
\hline$F_{d}(\mathrm{KN})$ & n 15 & 9,9 & 12,9 & 9,9 & 12,9 & 12,9 & 12,9 & - & 16,9 & 16,9 & 16,9 & \\
\hline & n 23 & - & & - & & & & - & - & - & - & 28,2 \\
\hline & n 31 & - & & & & & & & 34,9 & 34,9 & 34,9 & \\
\hline
\end{tabular}




\subsubsection{Treliças PRATT C30 telha fibrocimento}

Tabela 4.4 - Forças nos contraventamentos segundo os métodos simplificados (PRATT C30)

\begin{tabular}{|c|c|c|c|c|c|c|c|c|c|c|c|c|}
\hline \multicolumn{13}{|c|}{ GEOMETRIAS DAS TRELIÇAS } \\
\hline \multicolumn{2}{|c|}{ L (vão) } & 12 & 12 & 12 & 18 & 18 & 18 & 18 & 24 & 24 & 24 & 24 \\
\hline \multicolumn{2}{|c|}{ h (altura central) } & 1,5 & 1,5 & 1,5 & 2,25 & 2,25 & 2,25 & 2,25 & 3 & 3 & 3 & 3 \\
\hline \multirow{2}{*}{\multicolumn{2}{|c|}{$\begin{array}{c}\text { z (altura do pilar) } \\
\text { e (dist. entre treliças) }\end{array}$}} & 3 & 4 & 6 & 3 & 4 & 6 & 6 & 3 & 4 & 6 & 6 \\
\hline & & 3 & 3 & 3 & 3 & 3 & 3 & 4 & 3 & 3 & 3 & 4 \\
\hline \multicolumn{2}{|c|}{ Compressãomáx $_{(\mathrm{kN})}$} & 83,0 & 86,4 & 90,9 & 155,9 & 161,1 & 168,3 & 205,2 & 238,8 & 244,5 & 254,7 & 305,2 \\
\hline \multicolumn{13}{|c|}{ MÉTODO NBR 7190 (1997) } \\
\hline \multirow{6}{*}{$F_{d}(k N)$} & $\mathrm{F}_{1 \mathrm{~d}}$ & 0,6 & 0,6 & 0,6 & 1,0 & 1,1 & 1,1 & 1,4 & 1,6 & 1,6 & 1,7 & 2,0 \\
\hline & $\begin{array}{ll}\mathrm{n} & 7 \\
\end{array}$ & 2,6 & 2,7 & 2,8 & 4,9 & 5,0 & 5,2 & - & - & - & - & - \\
\hline & n 11 & - & - & - & - & - & - & 10,0 & - & - & - & 14,9 \\
\hline & n 15 & 5,5 & 5,8 & 6,1 & 10,4 & 10,7 & 11,2 & & 15,9 & 16,3 & 17,0 & - \\
\hline & n 23 & - & - & - & & & - & & & - & - & 31,2 \\
\hline & n 31 & - & & & & & & & 32,9 & 33,7 & 35,1 & \\
\hline \multicolumn{13}{|c|}{ MÉTODO EUROCODE 5} \\
\hline \multirow{6}{*}{$F_{d}(k N)$} & $\mathrm{F}_{1 \mathrm{~d}}$ & 1,7 & 1,7 & 1,8 & 3,1 & 3,2 & 3,4 & 4,1 & 4,8 & 4,9 & 5,1 & 6,1 \\
\hline & $\mathrm{n} 7$ & 7,7 & 8,1 & 8,5 & 14,6 & 15,0 & 15,7 & - & - & - & - & - \\
\hline & n 11 & - & - & - & - & - & - & 30,1 & - & - & - & 44,8 \\
\hline & n 15 & 16,6 & 17,3 & 18,2 & 31,2 & 32,2 & 33,7 & & 47,8 & 48,9 & 50,9 & - \\
\hline & n 23 & - & & - & & & & & & - & - & 93,6 \\
\hline & n 31 & - & & & & & & & 98,7 & 101,1 & 105,3 & - \\
\hline \multicolumn{13}{|c|}{ MÉTODO UNDERWOOD (2000) } \\
\hline \multirow{6}{*}{$F_{d}(k N)$} & $\mathrm{F}_{1 \mathrm{~d}}$ & 2,6 & 2,7 & 2,8 & 4,9 & 5,0 & 5,3 & 6,4 & 7,5 & 7,6 & 8,0 & 9,5 \\
\hline & $\begin{array}{ll}\mathrm{n} \quad 7 \\
\end{array}$ & 12,1 & 12,6 & 13,3 & 22,7 & 23,5 & 24,5 & - & - & - & - & - \\
\hline & n 11 & - & & - & & & & 47,0 & & - & - & 69,9 \\
\hline & n 15 & 25,9 & 27,0 & 28,4 & 48,7 & 50,3 & 52,6 & & 74,6 & 76,4 & 79,6 & - \\
\hline & n 23 & - & & - & & & - & & & - & - & 146,2 \\
\hline & n 31 & - & & & & & & & 154,2 & 157,9 & 164,5 & - \\
\hline \multicolumn{13}{|c|}{ MÉTODO SABS 0163} \\
\hline \multicolumn{2}{|c|}{ Compressão $_{\text {perm }}(\mathrm{kN})$} & & 38,2 & & & 84,4 & & 93,2 & & 139,2 & & 151,6 \\
\hline \multirow{6}{*}{$F_{d}(k N)$} & $\mathrm{F}_{1 \mathrm{~d}}$ & & 1,0 & & & 1,4 & & 1,6 & & 1,7 & & 1,9 \\
\hline & n 7 & 4,5 & 4,5 & 4,5 & 6,6 & 6,6 & 6,6 & - & - & - & - & - \\
\hline & n 11 & - & & - & & & & 11,4 & & - & - & 13,9 \\
\hline & n 15 & 9,6 & 14,1 & 9,6 & 14,1 & 14,1 & 14,1 & & 17,4 & 17,4 & 17,4 & \\
\hline & n 23 & - & - & - & - & & - & & - & - & - & 29,1 \\
\hline & n 31 & - & & & & & & & 36,0 & 36,0 & 36,0 & \\
\hline
\end{tabular}




\subsubsection{Treliças PRATT C50 telha metálica}

Tabela 4.5 - Forças nos contraventamentos segundo os métodos simplificados (PRATT C50 telha metálica)

\begin{tabular}{|c|c|c|c|c|c|c|c|c|c|c|c|c|}
\hline & & & & GIVE & RIA & DAs & 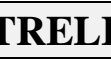 & AS & & & & \\
\hline $\mathrm{L}(\mathrm{v}$ & & 12 & 12 & 12 & 18 & 18 & 18 & 18 & 24 & 24 & 24 & 24 \\
\hline h (altura & ntral) & 1,5 & 1,5 & 1,5 & 2,25 & 2,25 & 2,25 & 2,25 & 3 & 3 & 3 & 3 \\
\hline z (altura & pilar) & 3 & 4 & 6 & 3 & 4 & 6 & 6 & 3 & 4 & 6 & 6 \\
\hline e (dist. ent & reliças) & 3 & 3 & 3 & 3 & 3 & 3 & 4 & 3 & 3 & 3 & 4 \\
\hline Compress & $\mathrm{x}(\mathrm{kN})$ & 65,9 & 68,3 & 74,0 & 116,8 & 120,7 & 126,5 & 160,3 & 185,3 & 190,8 & 202,5 & 244,6 \\
\hline & & & & MÉTC & DO N & BR 71 & $90(199$ & & & & & \\
\hline & $\mathrm{F}_{1 \mathrm{~d}}$ & 0,4 & 0,5 & 0,5 & 0,8 & 0,8 & 0,8 & 1,1 & 1,2 & 1,3 & 1,4 & 1,6 \\
\hline & $\begin{array}{ll}\mathrm{n} & 7\end{array}$ & 2,1 & 2,1 & 2,3 & 3,6 & 3,8 & 3,9 & - & - & - & - & - \\
\hline V) & n 11 & - & - & - & - & - & - & 7,8 & - & - & - & 12,0 \\
\hline & n 15 & 4,4 & 4,6 & 4,9 & 7,8 & 8,0 & 8,4 & - & 12,4 & 12,7 & 13,5 & - \\
\hline & n 23 & - & - & - & - & - & - & - & - & - & - & 25,0 \\
\hline & n 31 & - & - & - & - & - & - & - & 25,5 & 26,3 & 27,9 & - \\
\hline & & & & MÉT & DO I & URO & CODE & & & & & \\
\hline & $\mathrm{F}_{1 \mathrm{~d}}$ & 1,3 & 1,4 & 1,5 & 2,3 & 2,4 & 2,5 & 3,2 & 3,7 & 3,8 & 4,1 & 4,9 \\
\hline & $\begin{array}{ll}\mathrm{n} & 7\end{array}$ & 6,2 & 6,4 & 6,9 & 10,9 & 11,3 & 11,8 & - & - & - & - & - \\
\hline & n 11 & - & - & - & - & - & - & 23,5 & - & - & - & 35,9 \\
\hline & n 15 & 13,2 & 13,7 & 14,8 & 23,4 & 24,1 & 25,3 & - & 37,1 & 38,2 & 40,5 & - \\
\hline & n 23 & - & - & - & - & - & - & - & - & - & - & 75,0 \\
\hline & n 31 & - & - & - & - & - & - & - & 76,6 & 78,9 & 83,7 & - \\
\hline & & & MÉ & TOD & UN & RY & OD & 000) & & & & \\
\hline & $\mathrm{F}_{1 \mathrm{~d}}$ & 2,1 & 2,1 & 2,3 & 3,7 & 3,8 & 4,0 & 5,0 & 5,8 & 6,0 & 6,3 & 7,6 \\
\hline & $\begin{array}{ll}\mathrm{n} \quad 7\end{array}$ & 9,6 & 10,0 & 10,8 & 17,0 & 17,6 & 18,4 & - & - & - & - & - \\
\hline & n 11 & - & - & - & - & - & - & 36,7 & - & - & - & 56,1 \\
\hline & n 15 & 20,6 & 21,3 & 23,1 & 36,5 & 37,7 & 39,5 & - & 57,9 & 59,6 & 63,3 & - \\
\hline & n 23 & - & - & - & - & - & - & - & - & - & - & 117,2 \\
\hline & n 31 & - & - & - & - & - & - & - & 119,7 & 123,2 & 130,8 & - \\
\hline & & & & MÉ & OI & SAE & 0163 & & & & & \\
\hline Compressã & $\mathrm{rm}(\mathrm{kN})$ & & 20,1 & & & 41,3 & & 46,7 & & 86,0 & & 89,4 \\
\hline & $\mathrm{F}_{1 \mathrm{~d}}$ & & 0,5 & & & 0,7 & & 0,8 & & 1,1 & & 1,1 \\
\hline & n 7 & 2,3 & 2,3 & 2,3 & 3,2 & 3,2 & 3,2 & - & - & - & - & - \\
\hline & n 11 & - & - & - & - & - & - & 5,7 & - & - & - & 8,2 \\
\hline & n 15 & 5,0 & 6,9 & 5,0 & 6,9 & 6,9 & 6,9 & - & 10,8 & 10,8 & 10,8 & - \\
\hline & n 23 & - & - & - & - & - & - & - & - & - & - & 17,1 \\
\hline & n 31 & - & & 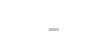 & & & - & - & 22,2 & 22,2 & 22,2 & - \\
\hline
\end{tabular}




\subsubsection{Treliças PRATT C50 telha cerâmica}

Tabela 4.6 - Forças nos contraventamentos segundo os métodos simplificados (PRATT C50 cerâmica)

\begin{tabular}{|c|c|c|c|c|c|c|c|}
\hline \multicolumn{8}{|c|}{ GEOMETRIAS DAS TRELIÇAS } \\
\hline \multicolumn{2}{|c|}{ L (vão) } & 12 & 12 & 12 & 18 & 18 & 18 \\
\hline \multicolumn{2}{|c|}{ h (altura central) } & 1,5 & 1,5 & 1,5 & 2,25 & 2,25 & 2,25 \\
\hline \multicolumn{2}{|c|}{ z (altura do pilar) } & 3 & 4 & 6 & 3 & 4 & 6 \\
\hline \multicolumn{2}{|c|}{ e (dist. entre treliças) } & 3 & 3 & 3 & 3 & 3 & 3 \\
\hline \multicolumn{2}{|c|}{ Compressãomáx $(k N)$} & 155,2 & 157,6 & 163,5 & 278,1 & 282,0 & 287,7 \\
\hline \multicolumn{8}{|c|}{ MÉTODO NBR 7190 (1997) } \\
\hline \multirow{3}{*}{$\mathbf{F}_{\mathbf{d}}(\mathbf{k N})$} & $\mathrm{F}_{1 \mathrm{~d}}$ & 1,0 & 1,1 & 1,1 & 1,9 & 1,9 & 1,9 \\
\hline & & 4,8 & 4,9 & 5,1 & 8,7 & 8,8 & 9,0 \\
\hline & 15 & 10,3 & 10,5 & 10,9 & 18,5 & 18,8 & 19,2 \\
\hline \multicolumn{8}{|c|}{ MÉTODO EUROCODE 5} \\
\hline \multirow{3}{*}{$\mathbf{F}_{\mathbf{d}}(\mathbf{k N})$} & $\mathrm{F}_{1 \mathrm{~d}}$ & 3,1 & 3,2 & 3,3 & 5,6 & 5,6 & 5,8 \\
\hline & & 14,5 & 14,7 & 15,3 & 26,0 & 26,3 & 26,9 \\
\hline & $\mathrm{n} \quad 15$ & 31,0 & 31,5 & 32,7 & 55,6 & 56,4 & 57,5 \\
\hline \multicolumn{8}{|c|}{ MÉTODO UNDERWOOD (2000) } \\
\hline \multirow{3}{*}{$F_{d}(\mathbf{k N})$} & $\mathrm{F}_{1 \mathrm{~d}}$ & 4,9 & 4,9 & 5,1 & 8,7 & 8,8 & 9,0 \\
\hline & & 22,6 & 23,0 & 23,8 & 40,6 & 41,1 & 42,0 \\
\hline & 15 & 48,5 & 49,3 & 51,1 & 86,9 & 88,1 & 89,9 \\
\hline \multicolumn{8}{|c|}{ MÉTODO SABS 0163} \\
\hline \multicolumn{2}{|c|}{ Compressão $_{\text {perm }}(\mathrm{kN})$} & \multicolumn{3}{|c|}{109,5} & \multicolumn{3}{|c|}{202,5} \\
\hline \multirow{3}{*}{$F_{d}(\mathbf{k N})$} & $F_{1 d}$ & \multicolumn{3}{|c|}{2,7} & \multicolumn{3}{|c|}{3,4} \\
\hline & & 12,8 & 12,8 & 12,8 & 15,8 & 15,8 & 15,8 \\
\hline & 15 & 27,4 & 33,8 & 27,4 & 33,8 & 33,8 & 33,8 \\
\hline
\end{tabular}




\subsection{Resultados dos modelos tridimensionais}

Os resultados dos modelos numéricos tridimensionais são divididos segundo os parâmetros adotados: tipo de treliça (Howe ou Pratt), classe de madeira (C30 ou C50), tipo de telha (fibrocimento, metálica ou cerâmica) e material do contraventamento (aço ou madeira).

\subsubsection{Modelos com contraventamento de cabos}

A seguir são mostrados os resultados dos modelos com barras de contraventamentos parametrizados com propriedades de cabos (E=195.000 MPa), resistentes apenas à tração.

As tabelas a seguir exibem os valores das forças axiais máximas nos banzos e no contraventamento constituído pelos cabos e terças. São mostrados os resultados dos modelos com todos os nós contraventados e dos modelos com um a cada dois nós contraventado.

Foram tomados os esforços máximos de compressão nos banzos e nas terças, e os máximos de tração nos cabos. 


\subsubsection{Modelos HOWE C50 telha fibrocimento}

Tabela 4.7 - Forças axiais máximas no banzo e no contraventamento (HOWE C50)

\begin{tabular}{|c|c|c|c|c|c|c|}
\hline \multicolumn{7}{|c|}{ FORÇA AXIAL MÁXIMA (kN) } \\
\hline \multirow{2}{*}{ Galpão } & \multicolumn{3}{|c|}{ Todos os nós contraventados } & \multicolumn{3}{|c|}{$\begin{array}{l}\text { Um cada dois nós } \\
\text { contraventado }\end{array}$} \\
\hline & $\begin{array}{l}\text { BANZO } \\
\text { (compr.) }\end{array}$ & $\begin{array}{l}\text { TERÇA } \\
\text { (compr.) }\end{array}$ & $\begin{array}{l}\text { CONTR. } \\
\text { (tração) }\end{array}$ & $\begin{array}{l}\text { BANZO } \\
\text { (compr.) }\end{array}$ & $\begin{array}{l}\text { TERÇA } \\
\text { (compr.) }\end{array}$ & $\begin{array}{l}\text { CONTR. } \\
\text { (tração) }\end{array}$ \\
\hline 1 & 92,3 & 12,4 & 8,4 & 90,0 & 12,3 & 8,5 \\
\hline 2 & 95,9 & 16,3 & 10,6 & 93,5 & 16,3 & 10,7 \\
\hline 3 & 100,4 & 23,5 & 15,0 & 98,0 & 23,5 & 14,4 \\
\hline 4 & 92,2 & 12,8 & 8,6 & 89,8 & 12,7 & 8,8 \\
\hline 5 & 95,8 & 16,9 & 11,8 & 93,5 & 16,8 & 11,1 \\
\hline 6 & 92,5 & 24,0 & 15,6 & 90,1 & 23,9 & 15,5 \\
\hline 7 & 149,7 & 12,8 & 17,6 & 146,1 & 16,1 & 22,7 \\
\hline 8 & 155,3 & 13,2 & 24,3 & 151,7 & 16,7 & 32,4 \\
\hline 9 & 162,4 & 15,4 & 34,8 & 158,8 & 17,5 & 43,7 \\
\hline 10 & 148,2 & 13,1 & 19,4 & 146,0 & 14,8 & 23,2 \\
\hline 11 & 153,8 & 13,6 & 24,2 & 151,7 & 15,4 & 32,0 \\
\hline 12 & 160,9 & 17,0 & 37,8 & 158,8 & 16,9 & 46,1 \\
\hline 13 & 209,2 & 19,0 & 38,0 & 208,3 & 19,1 & 44,8 \\
\hline 14 & 238,1 & 33,2 & 29,6 & 234,5 & 32,7 & 30,7 \\
\hline 15 & 245,6 & 35,8 & 43,5 & 242,2 & 43,1 & 38,2 \\
\hline 16 & 254,0 & 60,0 & 53,3 & 250,7 & 59,8 & 56,9 \\
\hline 17 & 307,3 & 59,3 & 53,2 & 304,4 & 59,2 & 56,4 \\
\hline 18 & 230,6 & 11,2 & 35,9 & 204,0 & 31,4 & 27,5 \\
\hline 19 & 244,0 & 33,6 & 40,8 & 238,5 & 35,5 & 40,4 \\
\hline 20 & 236,4 & 59,6 & 51,9 & 231,0 & 31,4 & 27,5 \\
\hline 21 & 293,4 & 59,2 & 51,5 & 291,1 & 58,9 & 55,3 \\
\hline
\end{tabular}

Observação: valores em módulo. 


\subsubsection{Modelos HOWE C30 telha fibrocimento}

Tabela 4.8 - Forças axiais máximas no banzo e no contraventamento (HOWE C30)

\begin{tabular}{|c|c|c|c|c|c|c|}
\hline \multicolumn{7}{|c|}{ FORÇA AXIAL MÁXIMA (kN) } \\
\hline \multirow{2}{*}{ Galpão } & \multicolumn{3}{|c|}{ Todos os nós contraventados } & \multicolumn{3}{|c|}{$\begin{array}{l}\text { Um cada dois nós } \\
\text { contraventado }\end{array}$} \\
\cline { 2 - 7 } & BANZO & TERÇA & CONTR. & BANZO & TERÇA & CONTR. \\
& (compr.) & (compr.) & (tração) & (compr.) & (compr.) & (tração) \\
\hline $\mathbf{1}$ & $\mathbf{8 8 , 2}$ & $\mathbf{1 2 , 4}$ & 9,4 & 85,9 & 12,4 & $\mathbf{1 0 , 1}$ \\
$\mathbf{2}$ & $\mathbf{9 1 , 7}$ & $\mathbf{1 6 , 3}$ & 12,0 & 89,4 & 16,3 & $\mathbf{1 2 , 5}$ \\
$\mathbf{3}$ & $\mathbf{9 6 , 2}$ & $\mathbf{2 3 , 5}$ & $\mathbf{1 6 , 9}$ & 93,9 & 23,5 & 16,9 \\
$\mathbf{4}$ & $\mathbf{8 8 , 1}$ & $\mathbf{1 2 , 8}$ & 9,6 & 85,9 & 12,7 & $\mathbf{1 0 , 2}$ \\
$\mathbf{5}$ & $\mathbf{9 1 , 7}$ & $\mathbf{1 6 , 9}$ & 12,3 & 89,5 & 16,8 & $\mathbf{1 2 , 8}$ \\
$\mathbf{6}$ & 91,6 & $\mathbf{2 3 , 8}$ & 17,2 & $\mathbf{9 2 , 4}$ & 23,8 & $\mathbf{1 7 , 6}$ \\
$\mathbf{7}$ & 156,8 & 9,7 & 17,7 & $\mathbf{1 5 7 , 5}$ & $\mathbf{1 6 , 7}$ & $\mathbf{1 9 , 9}$ \\
$\mathbf{8}$ & $\mathbf{1 6 6 , 7}$ & 14,2 & 25,3 & 163,2 & $\mathbf{1 7 , 4}$ & $\mathbf{2 9 , 9}$ \\
$\mathbf{9}$ & $\mathbf{1 7 3 , 8}$ & 17,1 & 36,4 & 170,3 & $\mathbf{1 8 , 2}$ & $\mathbf{4 1 , 9}$ \\
$\mathbf{1 0}$ & $\mathbf{1 5 9 , 0}$ & 14,7 & 20,1 & 157,3 & $\mathbf{1 5 , 7}$ & $\mathbf{2 2 , 0}$ \\
$\mathbf{1 1}$ & $\mathbf{1 6 4 , 7}$ & 15,2 & 25,3 & 163,0 & $\mathbf{1 6 , 3}$ & $\mathbf{2 9 , 5}$ \\
$\mathbf{1 2}$ & $\mathbf{1 7 1 , 7}$ & $\mathbf{1 8 , 7}$ & 39,6 & 170,1 & 17,0 & $\mathbf{4 4 , 4}$ \\
$\mathbf{1 3}$ & $\mathbf{2 1 4 , 3}$ & $\mathbf{1 9 , 9}$ & 38,9 & 213,8 & 18,7 & $\mathbf{4 4 , 6}$ \\
$\mathbf{1 4}$ & $\mathbf{2 4 1 , 2}$ & $\mathbf{3 3 , 2}$ & 30,7 & 236,5 & 32,7 & $\mathbf{3 2 , 9}$ \\
$\mathbf{1 5}$ & $\mathbf{2 4 8 , 6}$ & $\mathbf{4 3 , 5}$ & 37,1 & 244,2 & 43,2 & $\mathbf{4 0 , 7}$ \\
$\mathbf{1 6}$ & $\mathbf{2 5 7 , 0}$ & $\mathbf{6 0 , 0}$ & 55,3 & 252,8 & 59,8 & $\mathbf{6 0 , 5}$ \\
$\mathbf{1 7}$ & $\mathbf{3 1 4 , 9}$ & $\mathbf{5 9 , 3}$ & 54,8 & 312,1 & 59,2 & $\mathbf{5 9 , 3}$ \\
$\mathbf{1 8}$ & $\mathbf{2 3 9 , 6}$ & $\mathbf{3 1 , 9}$ & 29,6 & 234,2 & 31,4 & $\mathbf{2 9 , 6}$ \\
$\mathbf{1 9}$ & $\mathbf{2 4 7 , 2}$ & $\mathbf{4 0 , 9}$ & 34,8 & 241,5 & 40,4 & $\mathbf{3 7 , 9}$ \\
$\mathbf{2 0}$ & $\mathbf{2 3 9 , 6}$ & $\mathbf{5 9 , 6}$ & 53,8 & 234,2 & 59,3 & $\mathbf{6 0 , 0}$ \\
$\mathbf{2 1}$ & $\mathbf{2 9 5 , 2}$ & $\mathbf{5 9 , 2}$ & 52,9 & 292,9 & 59,0 & $\mathbf{5 7 , 6}$ \\
\hline
\end{tabular}




\subsubsection{Modelos PRATT C50 telha fibrocimento}

Tabela 4.9 - Forças axiais máximas no banzo e no contraventamento (PRATT C50)

\begin{tabular}{|c|c|c|c|c|c|c|}
\hline \multicolumn{7}{|c|}{ FORÇA AXIAL MÁXIMA (kN) } \\
\hline \multirow{2}{*}{ Galpão } & \multicolumn{3}{|c|}{ Todos os nós contraventados } & \multicolumn{3}{|c|}{$\begin{array}{l}\text { Um cada dois nós } \\
\text { contraventado }\end{array}$} \\
\hline & $\begin{array}{l}\text { BANZO } \\
\text { (compr.) }\end{array}$ & $\begin{array}{l}\text { TERÇA } \\
\text { (compr.) }\end{array}$ & $\begin{array}{l}\text { CONTR. } \\
\text { (tração) }\end{array}$ & $\begin{array}{l}\text { BANZO } \\
\text { (compr.) }\end{array}$ & $\begin{array}{l}\text { TERÇA } \\
\text { (compr.) }\end{array}$ & $\begin{array}{l}\text { CONTR. } \\
\text { (tração) }\end{array}$ \\
\hline 1 & 94,0 & 12,4 & 8,1 & 91,3 & 12,3 & 8,4 \\
\hline 2 & 97,7 & 16,3 & 10,4 & 95,1 & 16,3 & 10,6 \\
\hline 3 & 102,3 & 23,5 & 14,7 & 99,7 & 23,5 & 14,4 \\
\hline 4 & 93,8 & 12,8 & 8,5 & 91,2 & 12,7 & 8,7 \\
\hline 5 & 97,5 & 16,9 & 10,9 & 94,9 & 16,8 & 11,1 \\
\hline 6 & 94,2 & 24,0 & 15,6 & 91,6 & 23,9 & 15,4 \\
\hline 7 & 152,5 & 8,3 & 17,1 & 148,9 & 9,7 & 23,0 \\
\hline 8 & 158,2 & 11,0 & 24,0 & 154,6 & 12,4 & 32,8 \\
\hline 9 & 165,4 & 15,4 & 33,9 & 161,8 & 15,4 & 44,0 \\
\hline 10 & 153,0 & 8,9 & 18,9 & 150,0 & 11,9 & 23,6 \\
\hline 11 & 158,8 & 12,1 & 23,9 & 155,8 & 13,7 & 32,6 \\
\hline 12 & 166,1 & 17,0 & 37,1 & 163,2 & 17,0 & 46,5 \\
\hline 13 & 216,6 & 18,5 & 37,5 & 212,3 & 16,7 & 45,1 \\
\hline 14 & 246,0 & 33,3 & 29,1 & 242,3 & 32,8 & 29,7 \\
\hline 15 & 254,0 & 43,5 & 35,3 & 250,4 & 43,2 & 37,1 \\
\hline 16 & 263,2 & 60,0 & 52,8 & 259,5 & 59,8 & 55,8 \\
\hline 17 & 318,1 & 59,3 & 52,8 & 313,3 & 59,2 & 56,1 \\
\hline 18 & 243,2 & 32,1 & 26,4 & 238,9 & 31,6 & 26,6 \\
\hline 19 & 251,3 & 41,0 & 33,0 & 247,0 & 40,6 & 34,5 \\
\hline 20 & 243,2 & 59,6 & 51,5 & 238,9 & 59,4 & 55,8 \\
\hline 21 & 296,4 & 59,2 & 51,1 & 316,3 & 59,0 & 55,4 \\
\hline
\end{tabular}




\subsubsection{Modelos PRATT C30 telha fibrocimento}

Tabela 4.10 - Forças axiais máximas no banzo e no contraventamento (PRATT C30)

\begin{tabular}{|c|ccc|ccc|}
\hline \multicolumn{6}{|c|}{ FORÇA AXIAL MÁXIMA (kN) } \\
\hline \multirow{2}{*}{ Galpão } & Todos os nós contraventados & \multicolumn{3}{|c|}{$\begin{array}{c}\text { Um cada dois nós } \\
\text { contraventado }\end{array}$} \\
\cline { 2 - 7 } & BANZO & TERÇA & CONTR. & BANZO & TERÇA & CONTR. \\
& (compr.) & (compr.) & (tração) & (compr.) & (compr.) & (tração) \\
\hline $\mathbf{1}$ & $\mathbf{8 9 , 8}$ & $\mathbf{1 2 , 4}$ & 9,3 & 87,3 & 12,3 & $\mathbf{1 0 , 0}$ \\
$\mathbf{2}$ & $\mathbf{9 3 , 5}$ & $\mathbf{1 6 , 3}$ & 11,8 & 91,0 & 16,3 & $\mathbf{1 2 , 5}$ \\
$\mathbf{3}$ & $\mathbf{9 8 , 1}$ & $\mathbf{2 3 , 5}$ & 16,5 & 95,6 & 23,5 & $\mathbf{1 6 , 9}$ \\
$\mathbf{4}$ & $\mathbf{8 9 , 8}$ & $\mathbf{1 2 , 8}$ & 9,5 & 87,3 & 12,7 & $\mathbf{1 0 , 2}$ \\
$\mathbf{5}$ & $\mathbf{9 3 , 5}$ & $\mathbf{1 6 , 9}$ & 12,1 & 91,0 & 16,8 & $\mathbf{1 2 , 8}$ \\
$\mathbf{6}$ & $\mathbf{9 7 , 9}$ & $\mathbf{2 3 , 8}$ & 16,6 & 92,2 & 23,7 & $\mathbf{1 7 , 6}$ \\
$\mathbf{7}$ & $\mathbf{1 6 4 , 6}$ & $\mathbf{8 , 4}$ & 17,7 & 161,1 & 8,3 & $\mathbf{1 9 , 9}$ \\
$\mathbf{8}$ & $\mathbf{1 7 0 , 4}$ & $\mathbf{1 1 , 0}$ & 24,8 & 166,9 & 11,0 & $\mathbf{3 0 , 2}$ \\
$\mathbf{9}$ & $\mathbf{1 7 7 , 7}$ & $\mathbf{1 6 , 1}$ & 35,7 & 174,1 & 15,4 & $\mathbf{4 2 , 3}$ \\
$\mathbf{1 0}$ & $\mathbf{1 6 5 , 1}$ & $\mathbf{9 , 4}$ & 19,7 & 162,0 & 8,9 & $\mathbf{2 1 , 2}$ \\
$\mathbf{1 1}$ & $\mathbf{1 7 0 , 9}$ & $\mathbf{1 2 , 1}$ & 24,9 & 167,9 & 12,0 & $\mathbf{2 9 , 7}$ \\
$\mathbf{1 2}$ & $\mathbf{1 8 0 , 5}$ & $\mathbf{1 8 , 4}$ & 39,3 & 175,3 & 17,0 & $\mathbf{4 4 , 7}$ \\
$\mathbf{1 3}$ & $\mathbf{2 2 4 , 4}$ & $\mathbf{1 9 , 7}$ & 38,7 & 220,0 & 16,5 & $\mathbf{4 4 , 6}$ \\
$\mathbf{1 4}$ & $\mathbf{2 4 8 , 9}$ & $\mathbf{3 3 , 4}$ & 30,3 & 245,1 & 32,9 & $\mathbf{3 1 , 9}$ \\
$\mathbf{1 5}$ & $\mathbf{2 5 6 , 9}$ & $\mathbf{4 3 , 6}$ & 36,6 & 253,1 & 43,3 & $\mathbf{3 9 , 6}$ \\
$\mathbf{1 6}$ & $\mathbf{2 6 6 , 2}$ & $\mathbf{6 0 , 0}$ & 54,8 & 262,4 & 59,9 & $\mathbf{5 9 , 4}$ \\
$\mathbf{1 7}$ & $\mathbf{3 2 4 , 9}$ & $\mathbf{5 9 , 4}$ & 54,4 & 320,1 & 59,3 & $\mathbf{5 8 , 5}$ \\
$\mathbf{1 8}$ & $\mathbf{2 4 5 , 7}$ & $\mathbf{3 2 , 2}$ & 27,6 & 241,4 & 31,7 & $\mathbf{2 8 , 6}$ \\
$\mathbf{1 9}$ & $\mathbf{2 5 3 , 8}$ & $\mathbf{4 1 , 1}$ & 34,3 & 249,3 & 40,6 & $\mathbf{3 6 , 4}$ \\
$\mathbf{2 0}$ & $\mathbf{2 4 5 , 7}$ & $\mathbf{5 9 , 7}$ & 53,3 & 241,4 & 59,4 & $\mathbf{5 9 , 3}$ \\
$\mathbf{2 1}$ & $\mathbf{2 9 8 , 4}$ & $\mathbf{5 9 , 2}$ & 52,5 & 294,9 & 59,0 & $\mathbf{5 7 , 2}$ \\
\hline
\end{tabular}




\subsubsection{Modelos PRATT C50 telha metálica}

Tabela 4.11 - Forças axiais máximas no banzo e no contraventamento (PRATT C50 metálica)

\begin{tabular}{|c|c|c|c|c|c|c|}
\hline \multicolumn{7}{|c|}{ FORÇA AXIAL MÁXIMA (kN) } \\
\hline \multirow{2}{*}{ Galpão } & \multicolumn{3}{|c|}{ Todos os nós contraventados } & \multicolumn{3}{|c|}{$\begin{array}{l}\text { Um cada dois nós } \\
\text { contraventado }\end{array}$} \\
\hline & $\begin{array}{l}\text { BANZO } \\
\text { (compr.) }\end{array}$ & $\begin{array}{l}\text { TERÇA } \\
\text { (compr.) }\end{array}$ & $\begin{array}{l}\text { CONTR. } \\
\text { (tração) }\end{array}$ & $\begin{array}{l}\text { BANZO } \\
\text { (compr.) }\end{array}$ & $\begin{array}{l}\text { TERÇA } \\
\text { (compr.) }\end{array}$ & $\begin{array}{l}\text { CONTR. } \\
\text { (tração) }\end{array}$ \\
\hline 1 & 71,0 & 12,4 & 10,0 & 68,6 & 12,4 & 14,9 \\
\hline 2 & 75,9 & 16,3 & 12,2 & 72,2 & 16,3 & 17,3 \\
\hline 3 & 80,4 & 23,5 & 16,3 & 78,1 & 23,5 & 20,6 \\
\hline 4 & 72,2 & 12,7 & 10,1 & 70,2 & 12,7 & 14,7 \\
\hline 5 & 75,9 & 16,8 & 12,5 & 73,9 & 16,8 & 17,2 \\
\hline 6 & 105,7 & 23,9 & 17,0 & 100,1 & 23,9 & 20,9 \\
\hline 7 & 121,0 & 9,0 & 18,0 & 117,3 & 13,0 & 26,8 \\
\hline 8 & 126,7 & 11,3 & 24,9 & 123,1 & 15,7 & 36,4 \\
\hline 9 & 134,0 & 15,4 & 34,8 & 130,2 & 18,4 & 47,6 \\
\hline 10 & 121,0 & 9,5 & 19,6 & 118,0 & 16,2 & 27,4 \\
\hline 11 & 126,8 & 12,0 & 24,7 & 123,8 & 18,0 & 36,2 \\
\hline 12 & 134,1 & 17,0 & 37,2 & 131,1 & 20,8 & 49,9 \\
\hline 13 & 171,4 & 18,8 & 37,8 & 167,2 & 19,1 & 47,6 \\
\hline 14 & 195,0 & 33,2 & 28,8 & 191,1 & 32,7 & 31,4 \\
\hline 15 & 202,9 & 34,8 & 43,5 & 199,0 & 43,1 & 38,6 \\
\hline 16 & 212,7 & 60,0 & 51,5 & 208,8 & 59,8 & 61,6 \\
\hline 17 & 258,4 & 59,3 & 52,5 & 253,3 & 59,2 & 63,6 \\
\hline 18 & 192,1 & 32,0 & 26,2 & 187,8 & 31,4 & 27,7 \\
\hline 19 & 200,1 & 40,9 & 32,7 & 195,8 & 40,4 & 35,4 \\
\hline 20 & 192,1 & 59,6 & 50,6 & 187,8 & 59,3 & 60,5 \\
\hline 21 & 237,6 & 59,2 & 51,4 & 235,3 & 58,8 & 61,4 \\
\hline
\end{tabular}




\subsubsection{Modelos PRATT C50 telha cerâmica}

Tabela 4.12 - Forças axiais máximas no banzo e no contraventamento (PRATT C50 cerâmica)

\begin{tabular}{|c|c|c|c|c|c|c|}
\hline \multicolumn{7}{|c|}{ FORÇA AXIAL MÁXIMA (kN) } \\
\hline \multirow{3}{*}{ Galpão } & \multicolumn{3}{|c|}{ Todos os nós contraventados } & \multicolumn{3}{|c|}{$\begin{array}{r}\text { Um cada dois nós } \\
\text { contraventado }\end{array}$} \\
\cline { 2 - 7 } & BANZO & TERÇA & CONTR. & BANZO & TERÇA & CONTR. \\
& (compr.) & (compr.) & (tração) & (compr.) & (compr.) & (tração) \\
\hline $\mathbf{1}$ & $\mathbf{1 6 7 , 6}$ & 12,4 & $\mathbf{6 , 5}$ & 165,1 & $\mathbf{1 2 , 4}$ & 5,5 \\
$\mathbf{2}$ & $\mathbf{1 6 7 , 5}$ & 16,3 & $\mathbf{8 , 8}$ & 164,9 & $\mathbf{1 6 , 3}$ & 7,0 \\
$\mathbf{3}$ & $\mathbf{1 7 5 , 9}$ & 23,5 & $\mathbf{1 3 , 2}$ & 173,4 & $\mathbf{2 3 , 5}$ & 11,0 \\
$\mathbf{4}$ & $\mathbf{1 6 7 , 7}$ & 12,7 & $\mathbf{7 , 2}$ & 164,5 & $\mathbf{1 2 , 7}$ & 6,2 \\
$\mathbf{5}$ & $\mathbf{1 7 1 , 4}$ & 16,8 & $\mathbf{9 , 7}$ & 168,2 & $\mathbf{1 6 , 8}$ & 8,6 \\
$\mathbf{6}$ & $\mathbf{1 7 6 , 0}$ & $\mathbf{2 4 , 0}$ & $\mathbf{1 4 , 2}$ & 172,7 & 23,9 & 12,4 \\
$\mathbf{7}$ & $\mathbf{2 8 8 , 1}$ & 11,7 & $\mathbf{1 6 , 5}$ & 285,1 & $\mathbf{1 3 , 9}$ & 15,6 \\
$\mathbf{8}$ & $\mathbf{2 9 3 , 9}$ & 11,9 & 23,7 & 290,9 & $\mathbf{1 4 , 3}$ & $\mathbf{2 3 , 9}$ \\
$\mathbf{9}$ & $\mathbf{3 0 1 , 2}$ & $\mathbf{1 5 , 9}$ & 34,6 & 298,2 & 15,4 & $\mathbf{3 6 , 4}$ \\
$\mathbf{1 0}$ & $\mathbf{2 9 0 , 3}$ & 9,1 & 18,4 & $\mathbf{2 8 7 , 5}$ & $\mathbf{1 0 , 4}$ & $\mathbf{1 9 , 1}$ \\
$\mathbf{1 1}$ & $\mathbf{2 9 6 , 2}$ & $\mathbf{1 2 , 1}$ & 24,2 & 293,4 & 12,0 & $\mathbf{2 5 , 9}$ \\
$\mathbf{1 2}$ & $\mathbf{3 0 3 , 6}$ & $\mathbf{1 8 , 2}$ & 38,3 & 300,8 & 17,0 & $\mathbf{4 1 , 7}$ \\
\hline
\end{tabular}




\subsubsection{Modelo com contraventamento de madeira}

Para o modelo com contraventamento de madeira foram tomados apenas os esforços de compressão.

\subsubsection{Modelos PRATT C50 telha fibrocimento}

Tabela 4.13 - Forças axiais máximas no banzo e no contraventamento (PRATT C50)

\begin{tabular}{|c|c|c|c|c|c|c|}
\hline \multicolumn{7}{|c|}{ FORÇA AXIAL MÁXIMA (kN) } \\
\hline \multirow{2}{*}{ Galpão } & \multicolumn{3}{|c|}{ Todos os nós contraventados } & \multicolumn{3}{|c|}{$\begin{array}{l}\text { Um cada dois nós } \\
\text { contraventado }\end{array}$} \\
\hline & $\begin{array}{l}\text { BANZO } \\
\text { (compr.) }\end{array}$ & $\begin{array}{l}\text { TERÇA } \\
\text { (compr.) }\end{array}$ & $\begin{array}{l}\text { CONTR. } \\
\text { (compr.) }\end{array}$ & $\begin{array}{l}\text { BANZO } \\
\text { (compr.) }\end{array}$ & $\begin{array}{l}\text { TERÇA } \\
\text { (compr.) }\end{array}$ & $\begin{array}{l}\text { CONTR. } \\
\text { (compr.) }\end{array}$ \\
\hline 1 & 100,2 & 5,5 & 8,3 & 88,4 & 7,5 & 12,8 \\
\hline 2 & 103,6 & 7,4 & 10,1 & 91,8 & 8,9 & 13,3 \\
\hline 3 & 107,9 & 11,4 & 13,7 & 96,4 & 12,6 & 15,8 \\
\hline 4 & 101,0 & 5,1 & 8,6 & 88,5 & 6,6 & 12,4 \\
\hline 5 & 104,5 & 7,2 & 10,9 & 92,0 & 8,6 & 13,5 \\
\hline 6 & 101,4 & 11,4 & 14,3 & 88,9 & 12,9 & 16,1 \\
\hline 7 & 157,3 & 5,2 & 4,9 & 144,0 & 6,6 & 12,5 \\
\hline 8 & 162,8 & 7,0 & 5,0 & 149,6 & 8,3 & 12,9 \\
\hline 9 & 169,7 & 10,1 & 6,3 & 156,6 & 11,8 & 13,4 \\
\hline 10 & 164,2 & 4,8 & 6,4 & 145,3 & 7,0 & 17,0 \\
\hline 11 & 169,9 & 6,1 & 7,0 & 150,9 & 8,6 & 17,4 \\
\hline 12 & 177,1 & 9,0 & 9,2 & 157,9 & 11,3 & 18,0 \\
\hline 13 & 233,8 & 8,7 & 8,5 & 212,7 & 10,2 & 13,7 \\
\hline 14 & 263,5 & 16,3 & 20,5 & 239,4 & 17,5 & 24,2 \\
\hline 15 & 271,3 & 21,2 & 25,7 & 247,0 & 23,7 & 29,3 \\
\hline 16 & 280,2 & 29,6 & 34,2 & 255,8 & 29,3 & 37,3 \\
\hline 17 & 343,2 & 28,8 & 33,2 & 313,3 & 35,7 & 30,3 \\
\hline 18 & 261,0 & 15,5 & 20,6 & 235,4 & 16,7 & 24,3 \\
\hline 19 & 269,0 & 19,6 & 25,1 & 243,4 & 21,8 & 28,8 \\
\hline 20 & 261,1 & 28,7 & 35,0 & 236,1 & 33,2 & 38,5 \\
\hline 21 & 322,0 & 27,6 & 34,1 & 321,4 & 30,5 & 36,9 \\
\hline
\end{tabular}




\subsection{Considerações sobre os resultados}

Os valores considerados na análise dos resultados são os máximos - em negrito - entre os modelos com todos os nós contraventados e um a cada dois nós contraventado. Para os valores obtidos, em maioria, os esforços máximos nos banzos e nas terças foram dos modelos com todos os nós contraventados, e nas barras de contraventamento foram dos modelos com um a cada dois nós contraventados. 


\section{ANÁLISE DOS RESULTADOS}

Nas tabelas a seguir são apresentados os resultados obtidos nos métodos simplificados estudados e os obtidos nos modelos tridimensionais.

As tabelas apresentam os valores das forças máximas no contraventamento obtidas para cada método, em seguida apresentam um comparativo entre os dois métodos dado pela relação método simplificado sobre modelo tridimensional. São mostrados os valores de compressão máxima nos banzos e os valores de compressão máxima nos elementos constituintes do contraventamento, que para o caso de compressão são as terças ou elementos do contraventamento de madeira. As geometrias são divididas segundo o vão da treliça, a altura do pilar, o espaçamento entre treliças e o número de treliças paralelas contraventadas, representado pelo valor $n$.

A Figura 5.1 indica a natureza dos valores expostos nas tabelas.

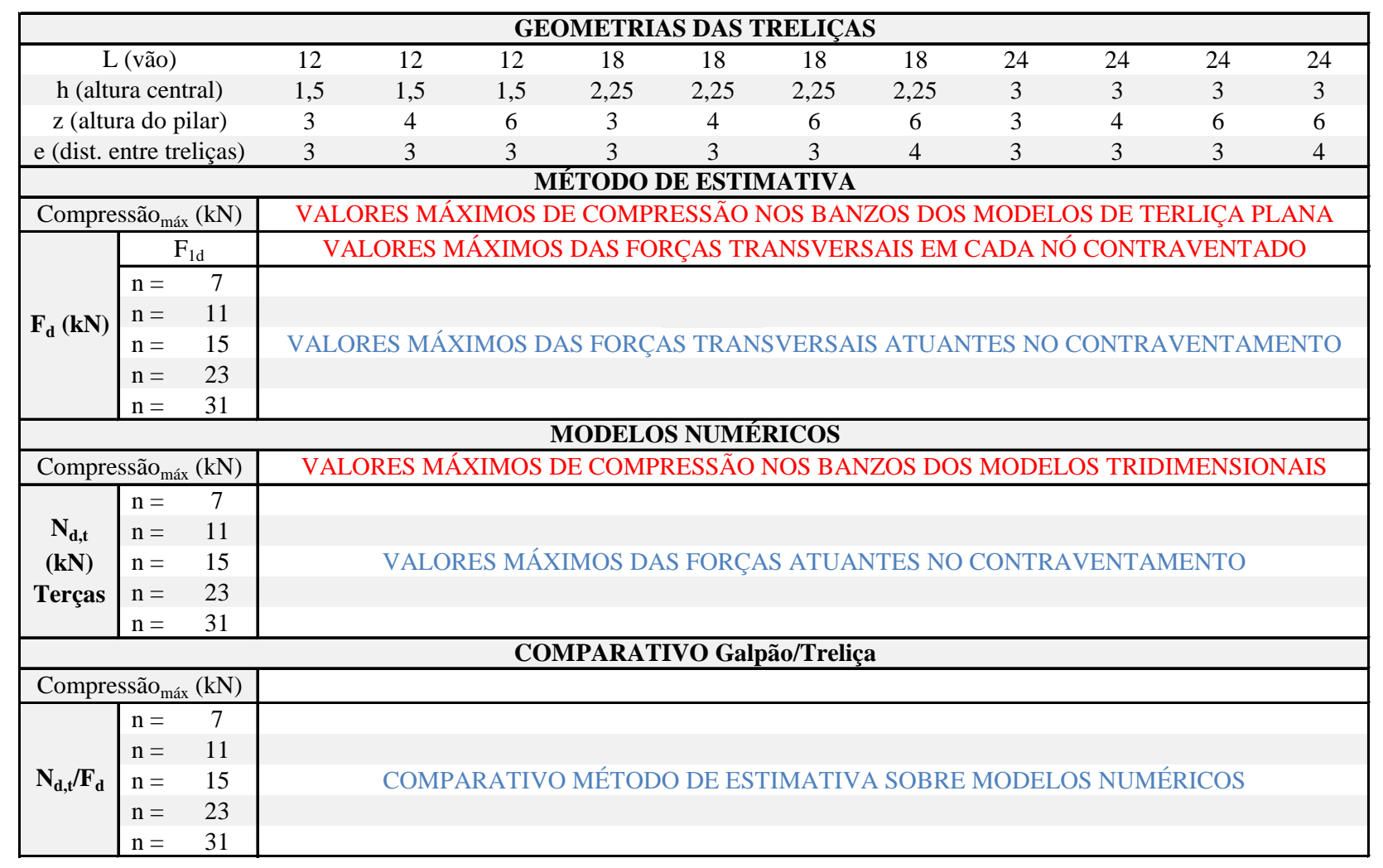

Figura 5.1 - Exemplo explicativos das tabelas

Os gráficos são apresentados os valores das tabelas em dois formatos para cada método estudado. Um gráfico de linhas suavizadas e quatro gráficos de colunas. 


\subsection{Treliças HOWE C50}

Tabela 5.1 - Comparativo entre o método da NBR 7190 (1997) e os modelos tridimensionais (HOWE C50)

\begin{tabular}{|c|c|c|c|c|c|c|c|c|c|c|c|c|}
\hline & & & GE & OME & ГRIAS & DAS 1 & RELI & ÇAS & & & & \\
\hline $\mathrm{L}(\mathrm{v}$ & & 12 & 12 & 12 & 18 & 18 & 18 & 18 & 24 & 24 & 24 & 24 \\
\hline h (altura & entral) & 1,5 & 1,5 & 1,5 & 2,25 & 2,25 & 2,25 & 2,25 & 3 & 3 & 3 & 3 \\
\hline z (altura & pilar) & 3 & 4 & 6 & 3 & 4 & 6 & 6 & 3 & 4 & 6 & 6 \\
\hline e (dist. ent & treliças) & 3 & 3 & 3 & 3 & 3 & 3 & 4 & 3 & 3 & 3 & 4 \\
\hline & & & & MÉTO & DO N & BR 719 & $0(1997$ & & & & & \\
\hline Compressã & máx $(\mathrm{kN})$ & 82,4 & 85,8 & 92,0 & 156,1 & 161,3 & 155,3 & 197,8 & 232,5 & 240,0 & 249,5 & 300,0 \\
\hline & $\mathrm{F}_{1 \mathrm{~d}}$ & 0,5 & 0,6 & 0,6 & 1,0 & 1,1 & 1,0 & 1,3 & 1,5 & 1,6 & 1,7 & 2,0 \\
\hline & $\mathrm{n}=7$ & 2,6 & 2,7 & 2,9 & 4,9 & 5,0 & 4,8 & - & - & - & - & - \\
\hline $\mathrm{F}_{d}(\mathbf{k N})$ & $\mathrm{n}=11$ & - & - & - & & - & - & 9,7 & - & - & & 14,7 \\
\hline $29(n i v)$ & $\mathrm{n}=15$ & 5,5 & 5,7 & 6,1 & 10,4 & 10,8 & 10,4 & & 15,5 & 16,0 & 16,6 & \\
\hline & $n=23$ & - & - & - & & - & - & - & - & & & 30,7 \\
\hline & $\mathrm{n}=31$ & - & - & - & - & - & - & - & 32,0 & 33,1 & 34,4 & - \\
\hline & & & & MODI & ELOS & NUMÉ & RICOS & & & & & \\
\hline Compressã & máx $(\mathrm{kN})$ & 92,3 & 95,9 & 100,4 & 149,7 & 155,3 & 162,4 & 209,2 & 238,1 & 245,6 & 254,0 & 307,3 \\
\hline & $\mathrm{n}=7$ & 12,4 & 16,3 & 23,5 & 16,1 & 16,7 & 17,5 & & & & & \\
\hline & $\mathrm{n}=11$ & - & & & & & & 19,1 & & & & 59,3 \\
\hline $\begin{array}{c}\text { Nd,t (kIV) } \\
\text { Tercas }\end{array}$ & $\mathrm{n}=15$ & 12,8 & 16,9 & 24,0 & 14,8 & 15,4 & 17,0 & - & 33,2 & 43,1 & 60,0 & - \\
\hline & $n=23$ & - & & & & & - & - & & & & 59,2 \\
\hline & $\mathrm{n}=31$ & - & & & & & & & 31,4 & 35,5 & 59,6 & \\
\hline & & & $\mathrm{CC}$ & MPAI & RATIV & O Gal & pão/Tr & eliça & & & & \\
\hline Compressã & máx $(\mathrm{kN})$ & 1,12 & 1,12 & 1,09 & 0,96 & 0,96 & 1,05 & 1,06 & 1,02 & 1,02 & 1,02 & 1,02 \\
\hline & $\mathrm{n}=7$ & 4,83 & 6,11 & 8,22 & 3,31 & 3,33 & 3,62 & & & & & \\
\hline & $\mathrm{n}=11$ & - & - & & & & - & 1,97 & - & & & 4,04 \\
\hline $\mathbf{N}_{\mathrm{d}, \mathrm{t}} / \mathbf{F}_{\mathbf{d}}$ & $\mathrm{n}=15$ & 2,32 & 2,95 & 3,91 & 1,42 & 1,43 & 1,64 & & 2,14 & 2,69 & 3,60 & \\
\hline & $\mathrm{n}=23$ & - & - & - & & & - & - & - & & & 1,93 \\
\hline & $\mathrm{n}=31$ & - & & & & & & & 0,98 & 1,07 & 1,73 & \\
\hline
\end{tabular}


Tabela 5.2 - Comparativo entre o método do EUROCODE 5 e os modelos tridimensionais (HOWE C50)

\begin{tabular}{|c|c|c|c|c|c|c|c|c|c|c|c|c|}
\hline \multicolumn{13}{|c|}{ GEOMETRIAS DAS TRELIÇAS } \\
\hline \multicolumn{2}{|c|}{ L (vão) } & 12 & 12 & 12 & 18 & 18 & 18 & 18 & 24 & 24 & 24 & 24 \\
\hline \multicolumn{2}{|c|}{ h (altura central) } & 1,5 & 1,5 & 1,5 & 2,25 & 2,25 & 2,25 & 2,25 & 3 & 3 & 3 & 3 \\
\hline \multicolumn{2}{|c|}{ z (altura do pilar) } & 3 & 4 & 6 & 3 & 4 & 6 & 6 & 3 & 4 & 6 & 6 \\
\hline \multicolumn{2}{|c|}{ e (dist. entre treliças) } & 3 & 3 & 3 & 3 & 3 & 3 & 4 & 3 & 3 & 3 & 4 \\
\hline \multicolumn{13}{|c|}{ MÉTODO EUROCODE 5} \\
\hline \multicolumn{2}{|c|}{ Compressãomáx $_{\text {mN) }}$} & 82,4 & 85,8 & 92,0 & 156,1 & 161,3 & 155,3 & 197,8 & 232,5 & 240,0 & 249,5 & 300,0 \\
\hline \multirow{6}{*}{$\mathbf{F}_{\mathbf{d}}(\mathbf{k N})$} & $\mathrm{F}_{1 \mathrm{~d}}$ & 1,6 & 1,7 & 1,8 & 3,1 & 3,2 & 3,1 & 4,0 & 4,6 & 4,8 & 5,0 & 6,0 \\
\hline & $\mathrm{n}=7$ & 7,7 & 8,0 & 8,6 & 14,6 & 15,1 & 14,5 & - & - & - & - & - \\
\hline & $\mathrm{n}=11$ & - & & & & & & 29,0 & & & & 44,0 \\
\hline & $\mathrm{n}=15$ & 16,5 & 17,2 & 18,4 & 31,2 & 32,3 & 31,1 & - & 46,5 & 48,0 & 49,9 & \\
\hline & $n=23$ & - & & & & - & & - & & - & - & 92,0 \\
\hline & $\mathrm{n}=31$ & - & - & - & - & - & - & - & 96,1 & 99,2 & 103,1 & - \\
\hline \multicolumn{13}{|c|}{ MODELOS NUMÉRICOS } \\
\hline \multicolumn{2}{|c|}{ Compressãomáx $_{(\mathrm{kN})}$} & 92,3 & 95,9 & 100,4 & 149,7 & 155,3 & 162,4 & 209,2 & 238,1 & 245,6 & 254,0 & 307,3 \\
\hline \multirow{5}{*}{$\begin{array}{c}\mathbf{N}_{\mathrm{d}, \mathrm{t}}(\mathbf{k N}) \\
\text { Terças }\end{array}$} & $\mathrm{n}=7$ & 12,4 & 16,3 & 23,5 & 16,1 & 16,7 & 17,5 & - & & - & - & \\
\hline & $\mathrm{n}=11$ & - & - & - & - & - & - & 19,1 & - & - & - & 59,3 \\
\hline & $\mathrm{n}=15$ & 12,8 & 16,9 & 24,0 & 14,8 & 15,4 & 17,0 & - & 33,2 & 43,1 & 60,0 & \\
\hline & $\mathrm{n}=23$ & - & - & - & - & - & - & - & - & - & - & 59,2 \\
\hline & $\mathrm{n}=31$ & - & - & - & - & - & - & - & 31,4 & 35,5 & 59,6 & - \\
\hline \multicolumn{13}{|c|}{ COMPARATIVO Galpão/Treliça } \\
\hline \multicolumn{2}{|c|}{ Compressão máx $(\mathrm{kN})$} & 1,12 & 1,12 & 1,09 & 0,96 & 0,96 & 1,05 & 1,06 & 1,02 & 1,02 & 1,02 & 1,02 \\
\hline \multirow{5}{*}{$\mathbf{N}_{\mathrm{d}, \mathrm{t}} / \mathbf{F}_{\mathbf{d}}$} & $\mathrm{n}=7$ & 1,61 & 2,04 & 2,74 & 1,10 & 1,11 & 1,21 & - & - & - & - & - \\
\hline & $\mathrm{n}=11$ & - & - & - & - & - & - & 0,66 & - & - & - & 1,35 \\
\hline & $\mathrm{n}=15$ & 0,77 & 0,98 & 1,30 & 0,47 & 0,48 & 0,55 & - & 0,71 & 0,90 & 1,20 & - \\
\hline & $\mathrm{n}=23$ & - & - & - & - & - & - & - & - & - & - & 0,64 \\
\hline & $\mathrm{n}=31$ & - & - & - & - & - & - & - & 0,33 & 0,36 & 0,58 & - \\
\hline
\end{tabular}


Tabela 5.3 - Comparativo entre o método de UNDERWOOD (2000) e os modelos tridimensionais (HOWE C50)

\begin{tabular}{|c|c|c|c|c|c|c|c|c|c|c|c|c|}
\hline \multicolumn{13}{|c|}{ GEOMETRIAS DAS TRELIÇAS } \\
\hline \multicolumn{2}{|c|}{ L (vão) } & 12 & 12 & 12 & 18 & 18 & 18 & 18 & 24 & 24 & 24 & 24 \\
\hline \multicolumn{2}{|c|}{ h (altura central) } & 1,5 & 1,5 & 1,5 & 2,25 & 2,25 & 2,25 & 2,25 & 3 & 3 & 3 & 3 \\
\hline \multicolumn{2}{|c|}{ z (altura do pilar) } & 3 & 4 & 6 & 3 & 4 & 6 & 6 & 3 & 4 & 6 & 6 \\
\hline \multicolumn{2}{|c|}{ e (dist. entre treliças) } & 3 & 3 & 3 & 3 & 3 & 3 & 4 & 3 & 3 & 3 & 4 \\
\hline \multicolumn{13}{|c|}{ MÉTODO UNDERWOOD (2000) } \\
\hline \multicolumn{2}{|c|}{ Compressãomáx $_{\text {ma }}(\mathrm{kN}$} & 82,4 & 85,8 & 92,0 & 156,1 & 161,3 & 155,3 & 197,8 & 232,5 & 240,0 & 249,5 & 300,0 \\
\hline \multirow{6}{*}{$F_{d}(k N)$} & $F_{1 d}$ & 2,6 & 2,7 & 2,9 & 4,9 & 5,0 & 4,9 & 6,2 & 7,3 & 7,5 & 7,8 & 9,4 \\
\hline & $\mathrm{n}=7$ & 12,0 & 12,5 & 13,4 & 22,8 & 23,5 & 22,6 & - & - & - & - & - \\
\hline & $\mathrm{n}=11$ & - & - & - & - & - & - & 45,3 & - & - & - & 68,7 \\
\hline & $\mathrm{n}=15$ & 25,7 & 26,8 & 28,8 & 48,8 & 50,4 & 48,5 & - & 72,7 & 75,0 & 78,0 & - \\
\hline & $\mathrm{n}=23$ & - & - & - & - & - & - & - & - & - & - & 143,7 \\
\hline & $\mathrm{n}=31$ & - & - & - & - & - & - & - & 150,1 & 155,0 & 161,1 & - \\
\hline \multicolumn{13}{|c|}{ MODELOS NUMÉRICOS } \\
\hline \multicolumn{2}{|c|}{ Compressãomáx $_{\text {mN }}(\mathrm{kN}$} & 92,3 & 95,9 & 100,4 & 149,7 & 155,3 & 162,4 & 209,2 & 238,1 & 245,6 & 254,0 & 307,3 \\
\hline \multirow{5}{*}{$\begin{array}{c}\mathbf{N}_{\mathrm{d}, \mathrm{t}}(\mathbf{k N}) \\
\text { Terças }\end{array}$} & $\mathrm{n}=7$ & 12,4 & 16,3 & 23,5 & 16,1 & 16,7 & 17,5 & - & - & - & - & - \\
\hline & $\mathrm{n}=11$ & - & - & - & - & - & - & 19,1 & - & - & - & 59,3 \\
\hline & $\mathrm{n}=15$ & 12,8 & 16,9 & 24,0 & 14,8 & 15,4 & 17,0 & - & 33,2 & 43,1 & 60,0 & - \\
\hline & $n=23$ & - & - & - & - & - & - & - & - & - & - & 59,2 \\
\hline & $\mathrm{n}=31$ & - & - & - & - & - & - & - & 31,4 & 35,5 & 59,6 & - \\
\hline \multicolumn{13}{|c|}{ COMPARATIVO Galpão/Treliça } \\
\hline \multicolumn{2}{|c|}{ Compressão máx $_{\text {mN) }}$} & 1,12 & 1,12 & 1,09 & 0,96 & 0,96 & 1,05 & 1,06 & 1,02 & 1,02 & 1,02 & 1,02 \\
\hline \multirow{5}{*}{$\mathbf{N}_{\mathrm{d}, \mathrm{t}} / \mathbf{F}_{\mathbf{d}}$} & $\mathrm{n}=7$ & 1,03 & 1,30 & 1,75 & 0,71 & 0,71 & 0,77 & - & - & - & - & - \\
\hline & $\mathrm{n}=11$ & - & - & - & - & - & - & 0,42 & - & - & - & 0,86 \\
\hline & $\mathrm{n}=15$ & 0,50 & 0,63 & 0,83 & $\mathbf{0 , 3 0}$ & $\mathbf{0 , 3 1}$ & 0,35 & - & 0,46 & 0,57 & 0,77 & - \\
\hline & $n=23$ & - & - & - & - & - & - & - & - & - & - & 0,41 \\
\hline & $\mathrm{n}=31$ & - & - & - & - & - & - & - & 0,21 & 0,23 & 0,37 & - \\
\hline
\end{tabular}


Tabela 5.4 - Comparativo entre o método do SABS 0163 e os modelos tridimensionais (HOWE C50)

\begin{tabular}{|c|c|c|c|c|c|c|c|c|c|c|c|c|}
\hline \multicolumn{13}{|c|}{ GEOMETRIAS DAS TRELIÇAS } \\
\hline \multicolumn{2}{|c|}{ L (vão) } & 12 & 12 & 12 & 18 & 18 & 18 & 18 & 24 & 24 & 24 & 24 \\
\hline \multicolumn{2}{|c|}{ h (altura central) } & 1,5 & 1,5 & 1,5 & 2,25 & 2,25 & 2,25 & 2,25 & 3 & 3 & 3 & 3 \\
\hline \multicolumn{2}{|c|}{ z (altura do pilar) } & 3 & 4 & 6 & 3 & 4 & 6 & 6 & 3 & 4 & 6 & 6 \\
\hline \multicolumn{2}{|c|}{ e (dist. entre treliças) } & 3 & 3 & 3 & 3 & 3 & 3 & 4 & 3 & 3 & 3 & 4 \\
\hline \multicolumn{13}{|c|}{ MÉTODO SABS 0163} \\
\hline \multicolumn{2}{|c|}{ Compressão $_{\text {perm }}(\mathrm{kN})$} & \multicolumn{3}{|c|}{39,3} & \multicolumn{3}{|c|}{77,0} & 85,8 & \multicolumn{3}{|c|}{134,3} & 146,3 \\
\hline \multirow{6}{*}{$\mathbf{F}_{\mathbf{d}}(\mathbf{k N})$} & $\mathrm{F}_{1 \mathrm{~d}}$ & \multicolumn{3}{|c|}{1,0} & \multicolumn{3}{|c|}{1,3} & 1,4 & \multicolumn{3}{|c|}{1,7} & 1,8 \\
\hline & $\mathrm{n}=7$ & 4,6 & 4,6 & 4,6 & 6,0 & 6,0 & 6,0 & - & - & - & - & - \\
\hline & $\mathrm{n}=11$ & - & - & - & - & - & - & 10,5 & - & - & - & 13,4 \\
\hline & $\mathrm{n}=15$ & 9,8 & 12,8 & 9,8 & 12,8 & 12,8 & 12,8 & & 16,8 & 16,8 & 16,8 & \\
\hline & $n=23$ & - & - & - & - & - & - & - & - & - & - & 28,0 \\
\hline & $\mathrm{n}=31$ & - & - & - & - & - & - & - & 34,7 & 34,7 & 34,7 & - \\
\hline \multicolumn{13}{|c|}{ MODELOS NUMÉRICOS } \\
\hline \multicolumn{2}{|c|}{ Compressão $_{\text {máx }}(\mathrm{kN})$} & 92,3 & 95,9 & 100,4 & 149,7 & 155,3 & 162,4 & 209,2 & 238,1 & 245,6 & 254,0 & 307,3 \\
\hline \multirow{5}{*}{$\begin{array}{c}\mathbf{N}_{\mathrm{d}, \mathrm{t}}(\mathbf{k N}) \\
\text { Terças }\end{array}$} & $\mathrm{n}=7$ & 12,4 & 16,3 & 23,5 & 16,1 & 16,7 & 17,5 & - & - & - & - & - \\
\hline & $\mathrm{n}=11$ & - & - & - & - & - & - & 19,1 & - & - & - & 59,3 \\
\hline & $\mathrm{n}=15$ & 12,8 & 16,9 & 24,0 & 14,8 & 15,4 & 17,0 & - & 33,2 & 43,1 & 60,0 & - \\
\hline & $n=23$ & - & - & - & - & - & - & - & - & - & - & 59,2 \\
\hline & $\mathrm{n}=31$ & - & - & - & - & - & - & - & 31,4 & 35,5 & 59,6 & - \\
\hline \multicolumn{13}{|c|}{ COMPARATIVO Galpão/Treliça } \\
\hline \multirow{5}{*}{$\mathbf{N}_{\mathrm{d}, \mathrm{t}} / \mathbf{F}_{\mathbf{d}}$} & $\mathrm{n}=7$ & 2,70 & 3,56 & 5,13 & 2,68 & 2,79 & 2,92 & & & - & - & \\
\hline & $\mathrm{n}=11$ & - & - & - & - & - & - & 1,82 & - & - & - & 4,42 \\
\hline & $\mathrm{n}=15$ & 1,30 & 1,32 & 2,44 & 1,15 & 1,20 & 1,32 & - & 1,98 & 2,57 & 3,57 & - \\
\hline & $n=23$ & - & - & - & - & - & - & - & - & - & - & 2,11 \\
\hline & $\mathrm{n}=31$ & - & - & - & - & - & - & - & $\mathbf{0 , 9 0}$ & 1,02 & 1,72 & - \\
\hline
\end{tabular}


Gráfico 1 - Linhas comparativas dos métodos (HOWE C50)

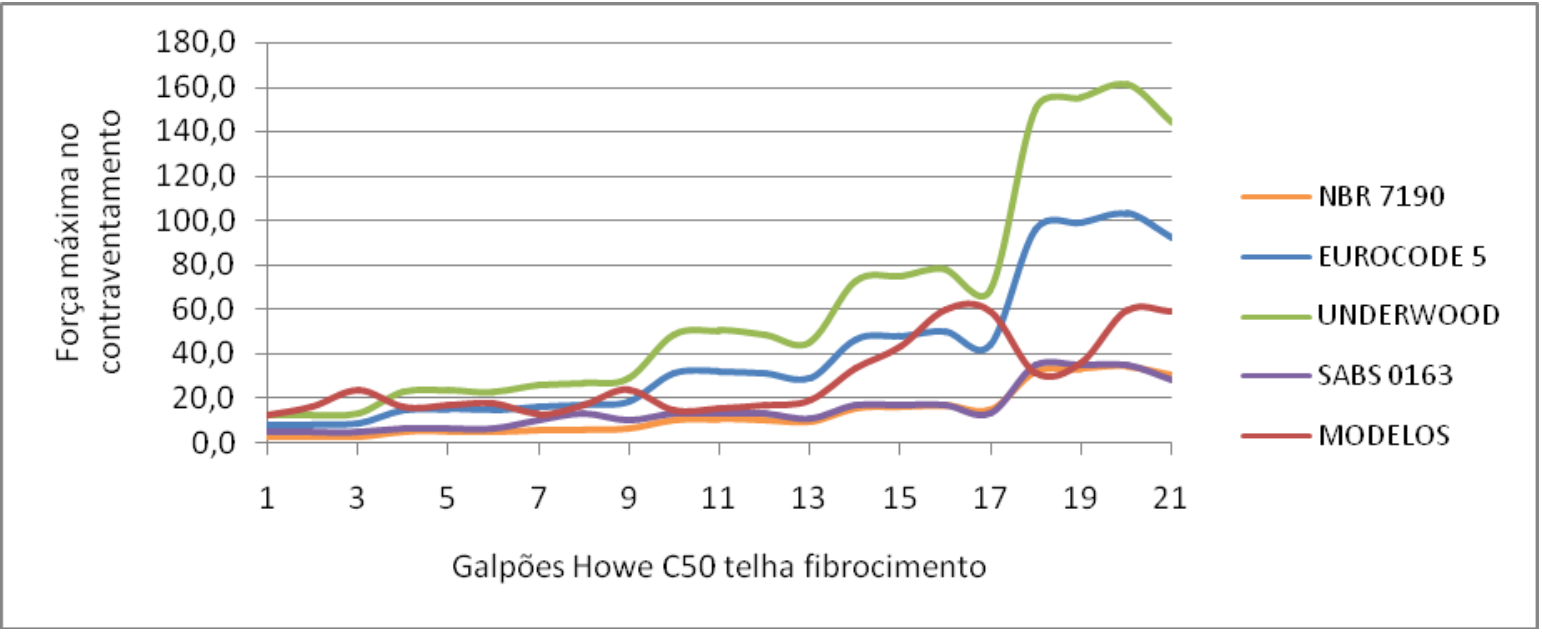

Gráfico 2 - Colunas comparativas do método NBR 7190 (HOWE C50)

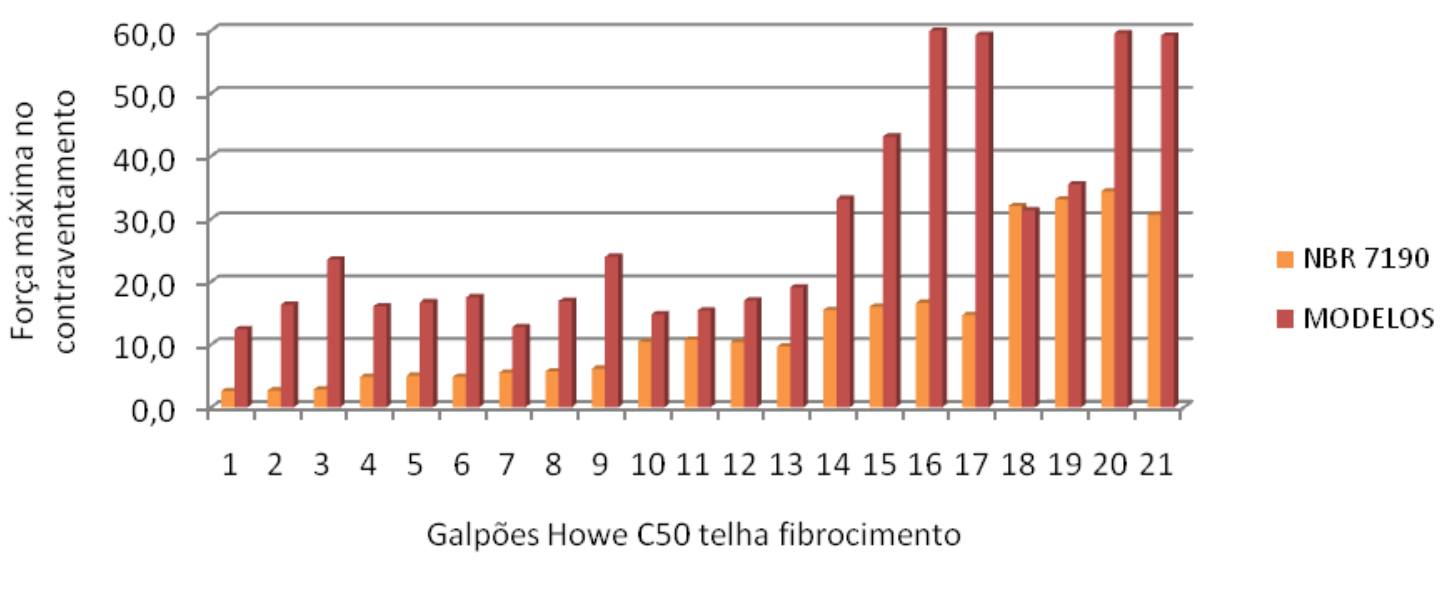

Gráfico 3 - Colunas comparativas do método EUROCODE 5 (HOWE C50)

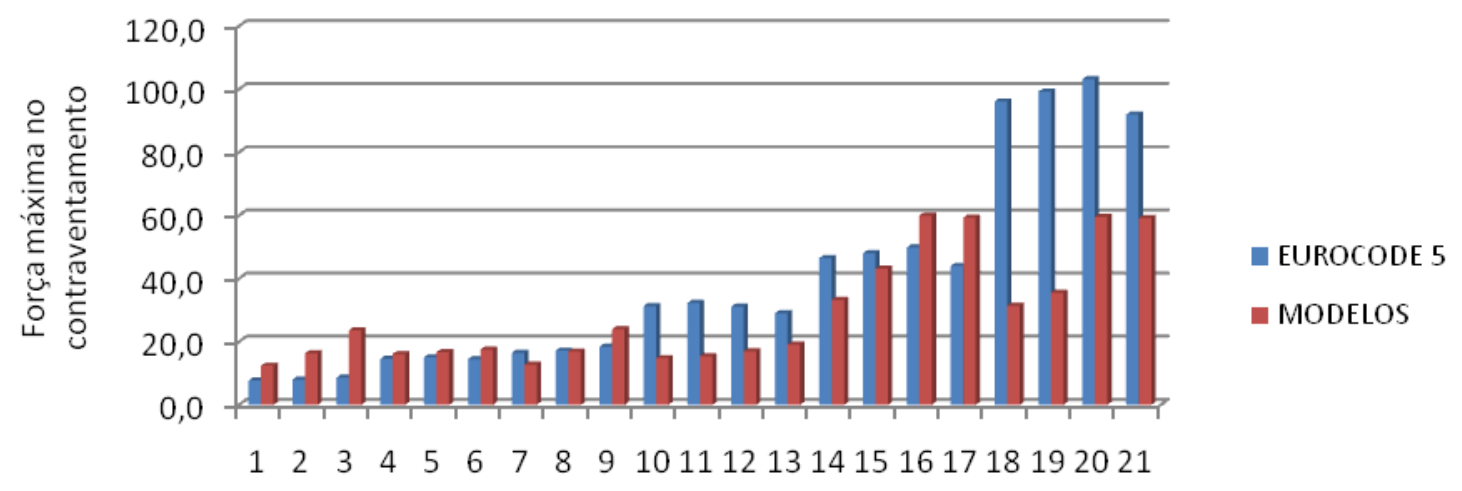

Galpões Howe C50 telha fibrocimento 
Gráfico 4 - Colunas comparativas do método UNDERWOOD (HOWE C50)

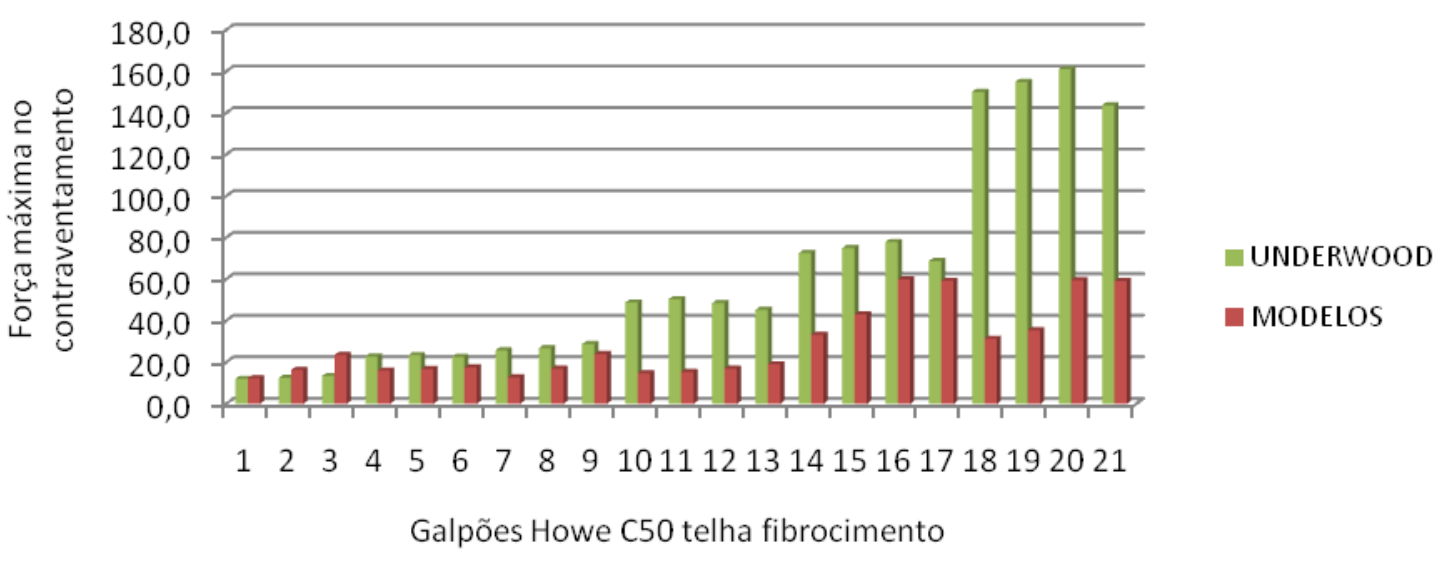

Gráfico 5 - Colunas comparativas do método SABS 0163 (HOWE C50)

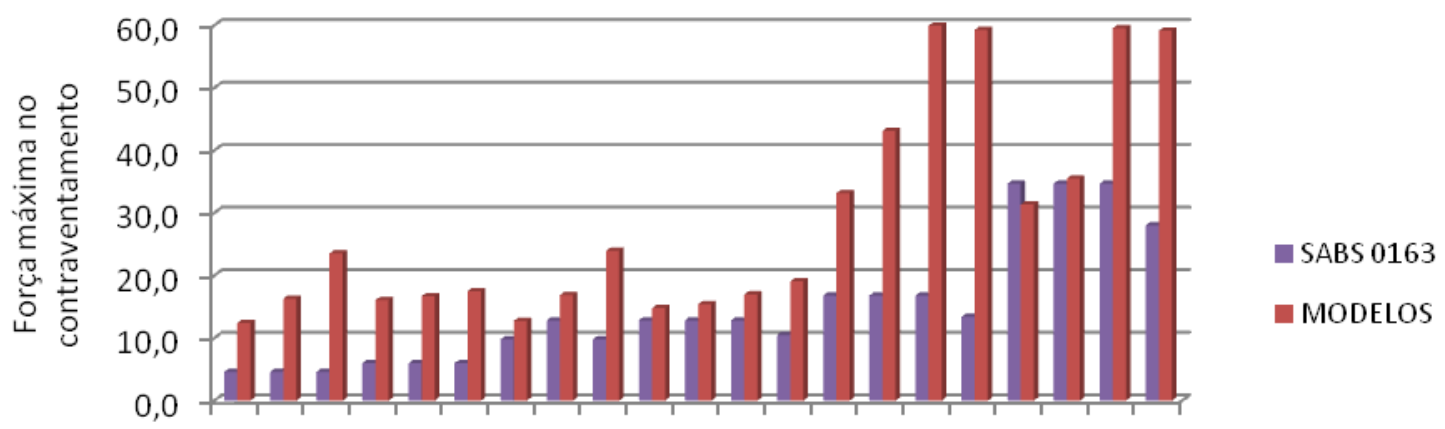

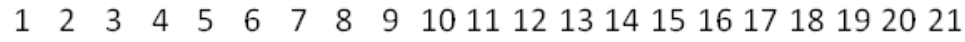

Galpões Howe C50 telha fibrocimento 


\subsection{Treliças HOWE C30}

Tabela 5.5 - Comparativo entre o método da NBR 7190 (1997) e os modelos tridimensionais (HOWE C30)

\begin{tabular}{|c|c|c|c|c|c|c|c|c|c|c|c|c|}
\hline & & & & 1 & $\boldsymbol{d}$ & $\mathbf{D A}$ & $\mathbf{E}$ & ÇAS & & & & \\
\hline $\mathrm{L}(\mathrm{v}$ & & 12 & 12 & 12 & 18 & 18 & 18 & 18 & 24 & 24 & 24 & 24 \\
\hline h (altur & ntral) & 1,5 & 1,5 & 1,5 & 2,25 & 2,25 & 2,25 & 2,25 & 3 & 3 & 3 & 3 \\
\hline $\mathrm{z}$ (alturc & pilar) & 3 & 4 & 6 & 3 & 4 & 6 & 6 & 3 & 4 & 6 & 6 \\
\hline e (dist. & iças) & 3 & 3 & 3 & . & 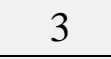 & 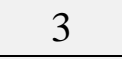 & 4 & 3 & 3 & 3 & 4 \\
\hline & & & & MÉ & 1 & 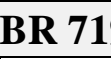 & $(199$ & & & & & \\
\hline Compres & náx $(\mathrm{kN})$ & 83,0 & 86,4 & 90,8 & 155,6 & 161,1 & 168,0 & 207,1 & 237,0 & 244,5 & 254,0 & 304,4 \\
\hline & $\mathrm{F}_{1 \mathrm{~d}}$ & 0,6 & 0,6 & 0,6 & 1,0 & 1,1 & 1,1 & 1,4 & 1,6 & 1,6 & 1,7 & 2,0 \\
\hline & $\mathrm{n}=7$ & 2,6 & 2,7 & 2,8 & 4,8 & 5,0 & 5,2 & - & - & - & - & - \\
\hline & $\mathrm{n}=11$ & - & - & - & - & - & - & 10,1 & - & - & - & 14,9 \\
\hline & $\mathrm{n}=15$ & 5,5 & 5,8 & 6,1 & 10,4 & 10,7 & 11,2 & - & 15,8 & 16,3 & 16,9 & - \\
\hline & $\mathrm{n}=23$ & - & - & - & - & - & - & - & - & - & - & 31,1 \\
\hline & $\mathrm{n}=31$ & - & - & - & - & - & - & - & 32,7 & 33,7 & 35,0 & - \\
\hline & & & & MOI & $\mathrm{O}$ & JUI & $\mathrm{RICO}$ & & & & & \\
\hline Compress & náx $(\mathrm{kN})$ & 88,2 & 91,7 & 96,2 & 159,0 & 166,7 & 173,8 & 214,3 & 241,2 & 248,6 & 257,0 & 314,9 \\
\hline & $\mathrm{n}=7$ & 12,4 & 16,3 & 23,5 & 16,7 & 17,4 & 18,2 & - & - & - & - & - \\
\hline & $\mathrm{n}=11$ & - & - & - & - & - & - & 19,9 & - & - & - & 59,3 \\
\hline $\begin{array}{c}N_{d, t}(K I N) \\
\text { Tercas }\end{array}$ & $\mathrm{n}=15$ & 12,8 & 16,9 & 23,8 & 15,7 & 16,3 & 18,7 & - & 33,2 & 43,5 & 60,0 & - \\
\hline & $n=23$ & - & - & - & - & - & - & - & - & - & - & 59,2 \\
\hline & $\mathrm{n}=31$ & - & - & - & - & - & 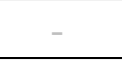 & - & 31,9 & 40,9 & 59,6 & - \\
\hline & & & $\mathrm{CO}$ & MPA & RATII & $\mathrm{OG}$ & pão/Tr & eliça & & & & \\
\hline Con & náx $(\mathrm{kN})$ & 1,06 & 1,06 & 1,06 & 1,02 & 1,0 & 1,03 & 1,03 & 1,02 & 1,02 & 1,01 & 1,03 \\
\hline & $\mathrm{n}=7$ & 4,80 & 6,06 & 8,32 & 3,45 & 3,47 & 3,48 & - & - & - & - & - \\
\hline & $\mathrm{n}=11$ & - & - & - & - & - & - & 1,97 & - & - & - & 3,98 \\
\hline $\mathbf{N}_{\mathrm{d}, \mathrm{t}} / \mathbf{F}_{\mathbf{d}}$ & $\mathrm{n}=15$ & 2,31 & 2,93 & 3,93 & 1,51 & 1,52 & 1,67 & & 2,10 & 2,67 & 3,54 & \\
\hline & $\mathrm{n}=23$ & - & - & - & - & - & - & - & - & - & - & 1,90 \\
\hline & $\mathrm{n}=31$ & 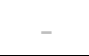 & - & - & - & - & & - & 0,98 & 1,21 & 1,70 & - \\
\hline
\end{tabular}


Tabela 5.6 - Comparativo entre o método do EUROCODE 5 e os modelos tridimensionais (HOWE C30)

\begin{tabular}{|c|c|c|c|c|c|c|c|c|c|c|c|c|}
\hline & & & GI & $\mathbf{M}$ & $\mathbf{R I}$ & DA & 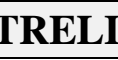 & ÇAS & & & & \\
\hline $\mathrm{L}($ & & 12 & 12 & 12 & 18 & 18 & 18 & 18 & 24 & 24 & 24 & 24 \\
\hline h (altura & entral) & 1,5 & 1,5 & 1,5 & 2,25 & 2,25 & 2,25 & 2,25 & 3 & 3 & 3 & 3 \\
\hline z (altur & pilar) & 3 & 4 & 6 & 3 & 4 & 6 & 6 & 3 & 4 & 6 & 6 \\
\hline e (dist. & liças) & 3 & 3 & . & 3 & 3 & U & 4 & 3 & 3 & 3 & 4 \\
\hline & & & & MÉr & $\overrightarrow{\mathrm{DC}}$ & JR & DDE & & & & & \\
\hline Compres & máx $(\mathrm{kN})$ & 83,0 & 86,4 & 90,8 & 155,6 & 161,1 & 168,0 & 207,1 & 237,0 & 244,5 & 254,0 & 304,4 \\
\hline & $\mathrm{F}_{1 \mathrm{~d}}$ & 1,7 & 1,7 & 1,8 & 3,1 & 3,2 & 3,4 & 4,1 & 4,7 & 4,9 & 5,1 & 6,1 \\
\hline & $\mathrm{n}=7$ & 7,7 & 8,1 & 8,5 & 14,5 & 15,0 & 15,7 & - & - & - & - & - \\
\hline & $\mathrm{n}=11$ & - & - & - & - & - & - & 30,4 & - & - & - & 44,6 \\
\hline & $\mathrm{n}=15$ & 16,6 & 17,3 & 18,2 & 31,1 & 32,2 & 33,6 & - & 47,4 & 48,9 & 50,8 & - \\
\hline & $\mathrm{n}=23$ & - & - & - & - & - & - & - & - & - & - & 93,3 \\
\hline & $\mathrm{n}=31$ & - & - & - & - & - & - & - & 98,0 & 101,1 & 105,0 & - \\
\hline & & & & MOI & LO & NUI & RIC & & & & & \\
\hline Compress & máx $(\mathrm{kN})$ & 88,2 & 91,7 & 96,2 & 159,0 & 166,7 & 173,8 & 214,3 & 241,2 & 248,6 & 257,0 & 314,9 \\
\hline & $\mathrm{n}=7$ & 12,4 & 16,3 & 23,5 & 16,7 & 17,4 & 18,2 & - & - & - & - & - \\
\hline & $\mathrm{n}=11$ & - & - & - & - & - & - & 19,9 & - & - & - & 59,3 \\
\hline $\mathbf{N}_{\text {d,t }}(\mathbf{k I V})$ & $\mathrm{n}=15$ & 12,8 & 16,9 & 23,8 & 15,7 & 16,3 & 18,7 & - & 33,2 & 43,5 & 60,0 & - \\
\hline & $\mathrm{n}=23$ & - & - & - & - & - & - & - & - & - & - & 59,2 \\
\hline & $\mathrm{n}=31$ & - & - & - & - & - & - & - & 31,9 & 40,9 & 59,6 & - \\
\hline & & & $\mathrm{CO}$ & MPAI & RATII & $\mathbf{O G}$ & ão/Tr & eliça & & & & \\
\hline Con & náx $(\mathrm{kN})$ & 1,06 & 1,06 & 1,06 & 1,02 & 1,03 & 1,03 & 1,03 & 1,02 & 1,02 & 1,01 & 1,03 \\
\hline & $\mathrm{n}=7$ & 1,60 & 2,02 & 2,77 & 1,15 & 1,16 & 1,16 & - & - & - & & - \\
\hline & $\mathrm{n}=11$ & - & - & - & - & - & - & 0,66 & - & - & - & 1,33 \\
\hline $\mathbf{N}_{\mathrm{d}, \mathrm{t}} / \mathbf{F}_{\mathbf{d}}$ & $\mathrm{n}=15$ & 0,77 & 0,98 & 1,31 & 0,50 & 0,51 & 0,56 & - & 0,70 & 0,89 & 1,18 & - \\
\hline & $\mathrm{n}=23$ & - & - & - & - & - & - & - & - & - & - & 0,63 \\
\hline & $\mathrm{n}=31$ & - & - & 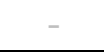 & - & - & - & - & 0,33 & 0,40 & 0,57 & - \\
\hline
\end{tabular}


Tabela 5.7 - Comparativo entre o método de UNDERWOOD (2000) e os modelos tridimensionais (HOWE C30)

\begin{tabular}{|c|c|c|c|c|c|c|c|c|c|c|c|c|}
\hline \multicolumn{13}{|c|}{ GEOMETRIAS DAS TRELIÇAS } \\
\hline \multicolumn{2}{|c|}{ L (vão) } & 12 & 12 & 12 & 18 & 18 & 18 & 18 & 24 & 24 & 24 & 24 \\
\hline \multicolumn{2}{|c|}{ h (altura central) } & 1,5 & 1,5 & 1,5 & 2,25 & 2,25 & 2,25 & 2,25 & 3 & 3 & 3 & 3 \\
\hline \multicolumn{2}{|c|}{ z (altura do pilar) } & 3 & 4 & 6 & 3 & 4 & 6 & 6 & 3 & 4 & 6 & 6 \\
\hline \multicolumn{2}{|c|}{ e (dist. entre treliças) } & 3 & 3 & 3 & 3 & 3 & 3 & 4 & 3 & 3 & 3 & 4 \\
\hline \multicolumn{13}{|c|}{ MÉTODO UNDERWOOD (2000) } \\
\hline \multicolumn{2}{|c|}{ Compressão máx $_{\text {mN) }}$} & 83,0 & 86,4 & 90,8 & 155,6 & 161,1 & 168,0 & 207,1 & 237,0 & 244,5 & 254,0 & 304,4 \\
\hline \multirow{6}{*}{$F_{d}(\mathbf{k N})$} & $F_{1 d}$ & 2,6 & 2,7 & 2,8 & 4,9 & 5,0 & 5,3 & 6,5 & 7,4 & 7,6 & 7,9 & 9,5 \\
\hline & $\mathrm{n}=7$ & 12,1 & 12,6 & 13,2 & 22,7 & 23,5 & 24,5 & - & - & - & - & - \\
\hline & $\mathrm{n}=11$ & - & - & - & - & - & - & 47,5 & - & - & - & 69,8 \\
\hline & $\mathrm{n}=15$ & 25,9 & 27,0 & 28,4 & 48,6 & 50,3 & 52,5 & - & 74,1 & 76,4 & 79,4 & - \\
\hline & $\mathrm{n}=23$ & - & - & - & - & - & - & - & - & - & - & 145,9 \\
\hline & $\mathrm{n}=31$ & - & - & - & - & - & - & - & 153,1 & 157,9 & 164,0 & - \\
\hline \multicolumn{13}{|c|}{ MODELOS NUMÉRICOS } \\
\hline \multicolumn{2}{|c|}{ Compressão $_{\text {máx }}(\mathrm{kN})$} & 88,2 & 91,7 & 96,2 & 159,0 & 166,7 & 173,8 & 214,3 & 241,2 & 248,6 & 257,0 & 314,9 \\
\hline \multirow{5}{*}{$\begin{array}{c}\mathbf{N}_{\mathrm{d}, \mathrm{t}}(\mathbf{k N}) \\
\text { Terças }\end{array}$} & $\mathrm{n}=7$ & 12,4 & 16,3 & 23,5 & 16,7 & 17,4 & 18,2 & - & - & - & - & - \\
\hline & $\mathrm{n}=11$ & - & - & - & - & - & - & 19,9 & - & - & - & 59,3 \\
\hline & $\mathrm{n}=15$ & 12,8 & 16,9 & 23,8 & 15,7 & 16,3 & 18,7 & - & 33,2 & 43,5 & 60,0 & - \\
\hline & $n=23$ & - & - & - & - & - & - & - & - & - & - & 59,2 \\
\hline & $\mathrm{n}=31$ & - & - & - & - & - & - & - & 31,9 & 40,9 & 59,6 & - \\
\hline \multicolumn{13}{|c|}{ COMPARATIVO Galpão/Treliça } \\
\hline \multicolumn{2}{|c|}{ Compressão $_{\text {máx }}(\mathrm{kN})$} & 1,06 & 1,06 & 1,06 & 1,02 & 1,03 & 1,03 & 1,03 & 1,02 & 1,02 & 1,01 & 1,03 \\
\hline \multirow{5}{*}{$\mathbf{N}_{\mathbf{d}, \mathrm{t}} / \mathbf{F}_{\mathbf{d}}$} & $\mathrm{n}=7$ & 1,02 & 1,29 & 1,77 & 0,74 & 0,74 & 0,74 & - & - & - & - & - \\
\hline & $\mathrm{n}=11$ & - & - & - & - & - & - & 0,42 & - & - & - & 0,85 \\
\hline & $\mathrm{n}=15$ & 0,49 & 0,63 & 0,84 & 0,32 & 0,32 & 0,36 & - & 0,45 & 0,57 & 0,76 & - \\
\hline & $n=23$ & - & - & - & - & - & - & - & - & - & - & 0,41 \\
\hline & $\mathrm{n}=31$ & - & - & - & - & - & - & - & 0,21 & 0,26 & 0,36 & - \\
\hline
\end{tabular}


Tabela 5.8 - Comparativo entre o método do SABS 0163 e os modelos tridimensionais (HOWE C30)

\begin{tabular}{|c|c|c|c|c|c|c|c|c|c|c|c|c|}
\hline & & & GE & OM & RI & DA & IRE & ÇAS & & & & \\
\hline $\mathrm{L}(\mathrm{v}$ & & 12 & 12 & 12 & 18 & 18 & 18 & 18 & 24 & 24 & 24 & 24 \\
\hline h (altura & entral) & 1,5 & 1,5 & 1,5 & 2,25 & 2,25 & 2,25 & 2,25 & 3 & 3 & 3 & 3 \\
\hline z (altura & pilar) & 3 & 4 & 6 & 3 & 4 & 6 & 6 & 3 & 4 & 6 & 6 \\
\hline e (dist. & liças) & 3 & 3 & U & 3 & 3 & 3 & 4 & 3 & 3 & 3 & 4 \\
\hline & & & & & OI & SA & 0163 & & & & & \\
\hline Compressã & erm $(\mathrm{kN})$ & & 38,1 & & & 84,1 & & 95,2 & & 138,8 & & 150,8 \\
\hline & $\mathrm{F}_{1 \mathrm{~d}}$ & & 1,0 & & & 1,4 & & 1,6 & & 1,7 & & 1,9 \\
\hline & $\mathrm{n}=7$ & 4,4 & 4,4 & 4,4 & 6,5 & 6,5 & 6,5 & - & - & - & - & - \\
\hline J) & $\mathrm{n}=11$ & - & - & - & - & - & - & 11,6 & - & - & - & 13,8 \\
\hline & $\mathrm{n}=15$ & 9,5 & 14,0 & 9,5 & 14,0 & 14,0 & 14,0 & & 17,4 & 17,4 & 17,4 & \\
\hline & $n=23$ & - & - & - & - & - & - & - & - & - & - & 28,9 \\
\hline & $\mathrm{n}=31$ & - & - & - & - & - & - & - & 35,9 & 35,9 & 35,9 & - \\
\hline & & & & MOD & ILOS & NUN & RICO & & & & & \\
\hline Compressã & máx $(\mathrm{kN})$ & 88,2 & 91,7 & 96,2 & 159,0 & 166,7 & 173,8 & 214,3 & 241,2 & 248,6 & 257,0 & 314,9 \\
\hline & $\mathrm{n}=7$ & 12,4 & 16,3 & 23,5 & 16,7 & 17,4 & 18,2 & - & - & - & - & - \\
\hline & $\mathrm{n}=11$ & - & - & - & - & - & - & 19,9 & - & - & - & 59,3 \\
\hline $\mathbf{N}_{\mathrm{d}, \mathrm{t}}(\mathrm{kN})$ & $\mathrm{n}=15$ & 12,8 & 16,9 & 23,8 & 15,7 & 16,3 & 18,7 & & 33,2 & 43,5 & 60,0 & \\
\hline & $\mathrm{n}=23$ & - & - & - & - & - & - & - & - & - & - & 59,2 \\
\hline & $\mathrm{n}=31$ & - & - & - & - & - & - & - & 31,9 & 40,9 & 59,6 & - \\
\hline & & & $\mathrm{CO}$ & MPA & RATIV & $\mathbf{O} \mathbf{G}$ & pão/T & eliça & & & & \\
\hline & $\mathrm{n}=7$ & 2,79 & 3,67 & 5,29 & 2,55 & 2,66 & 2,78 & - & - & - & - & - \\
\hline & $\mathrm{n}=11$ & - & - & - & - & - & - & 1,71 & - & - & - & 4,29 \\
\hline $\mathbf{N}_{\mathrm{d}, \mathrm{t}} / \mathbf{F}_{\mathbf{d}}$ & $\mathrm{n}=15$ & 1,34 & 1,21 & 2,50 & 1,12 & 1,16 & 1,33 & & 1,91 & 2,51 & 3,46 & \\
\hline & $n=23$ & - & - & - & - & - & - & - & - & - & - & 2,05 \\
\hline & $\mathrm{n}=31$ & - & - & - & - & - & - & - & 0,89 & 1,14 & 1,66 & - \\
\hline
\end{tabular}


Gráfico 6 - Linhas comparativas dos métodos (HOWE C30)

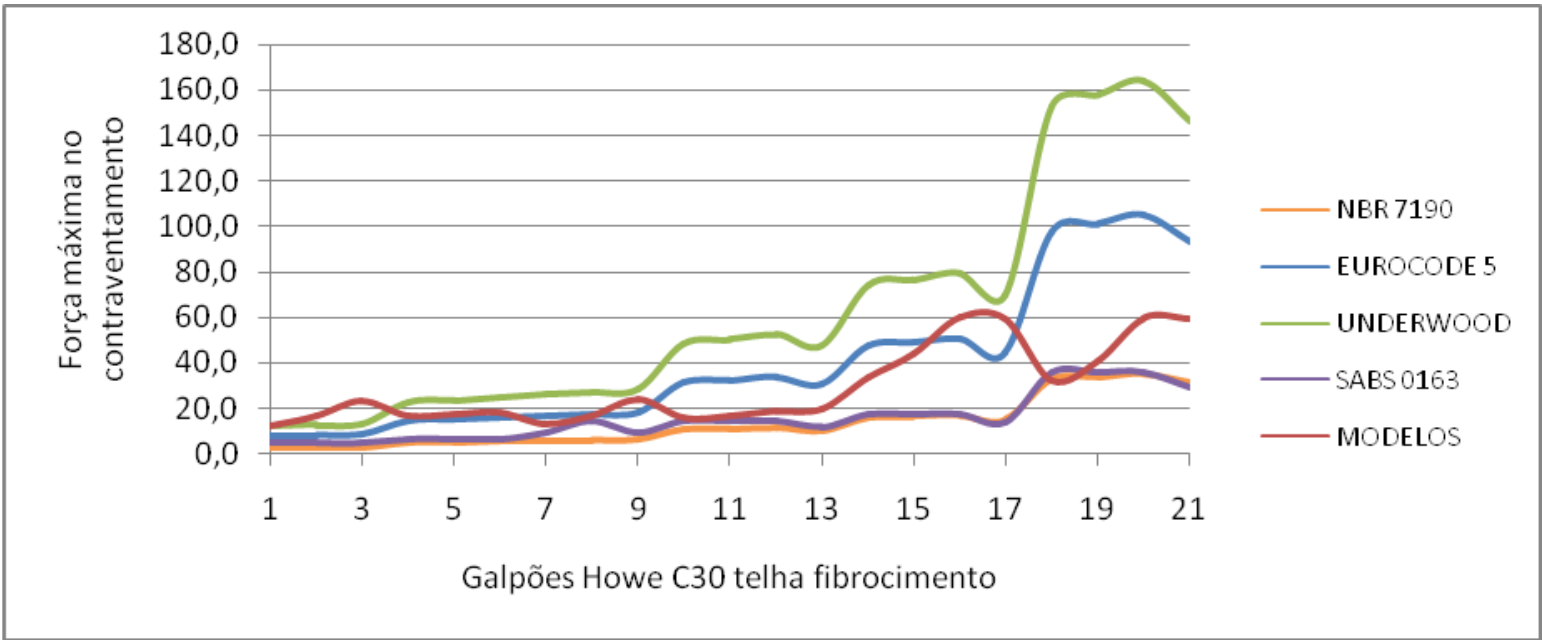

Gráfico 7 - Colunas comparativas do método NBR 7190 (HOWE C30)

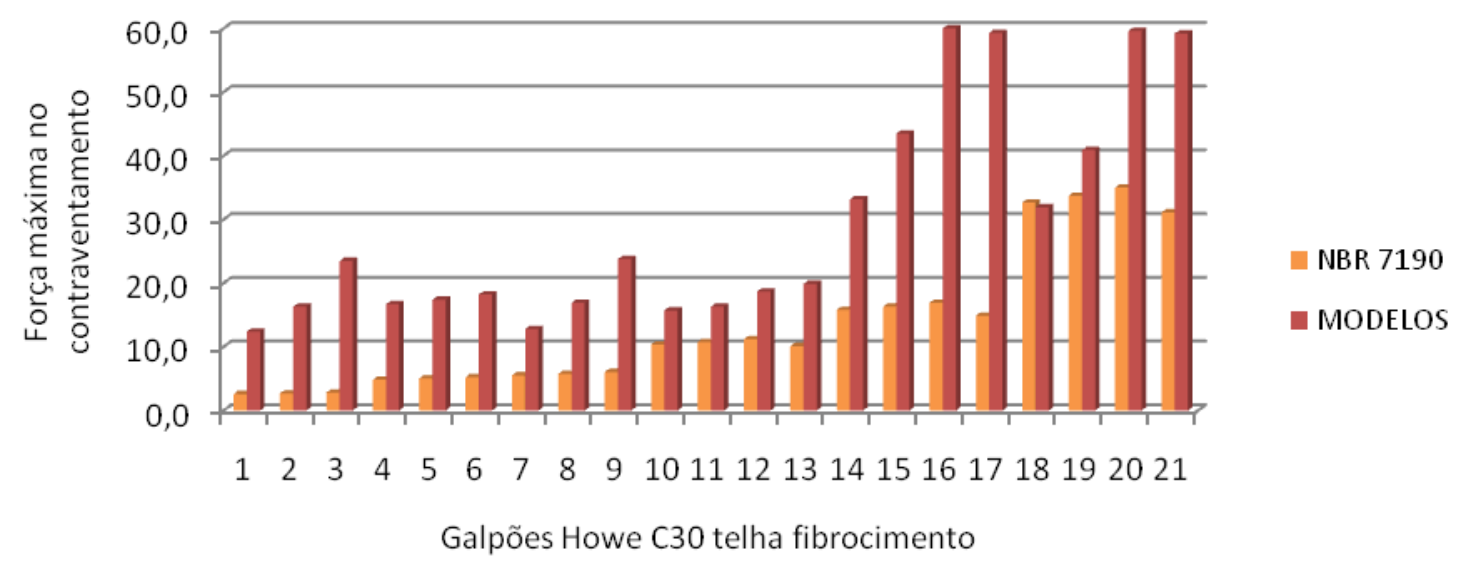

Gráfico 8 - Colunas comparativas do método EUROCODE 5 (HOWE C30)

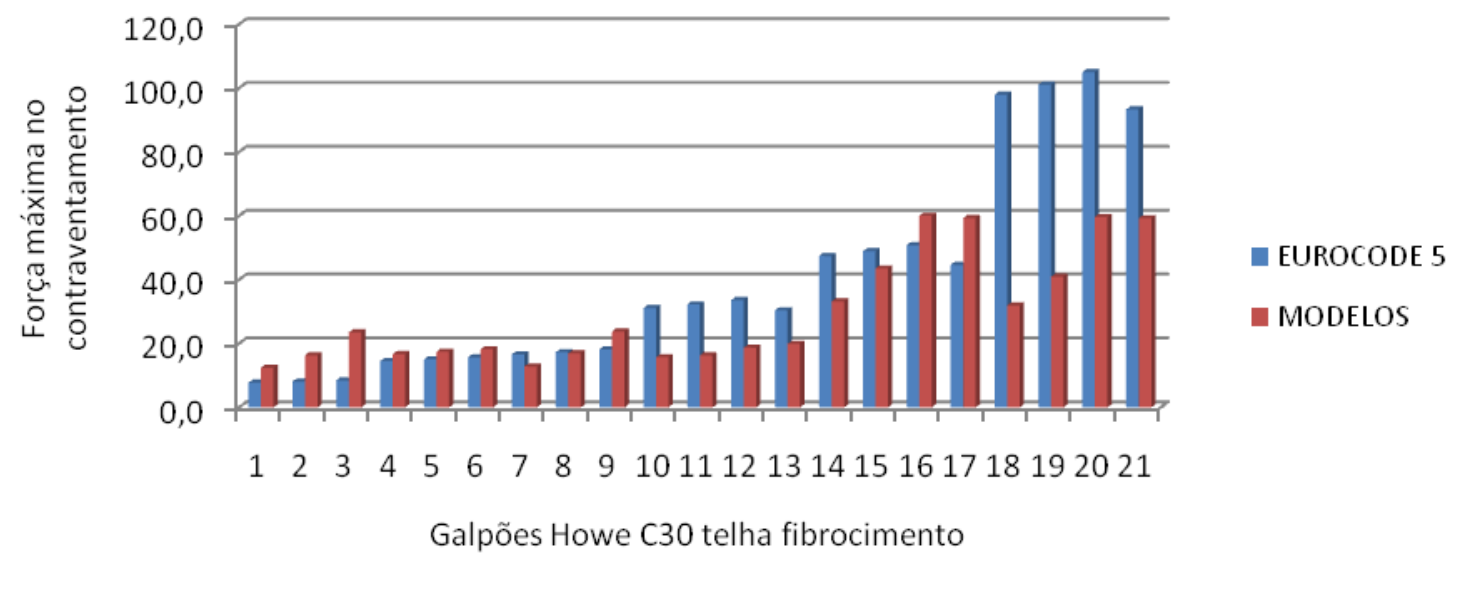


Gráfico 9 - Colunas comparativas do método UNDERWOOD (HOWE C30)

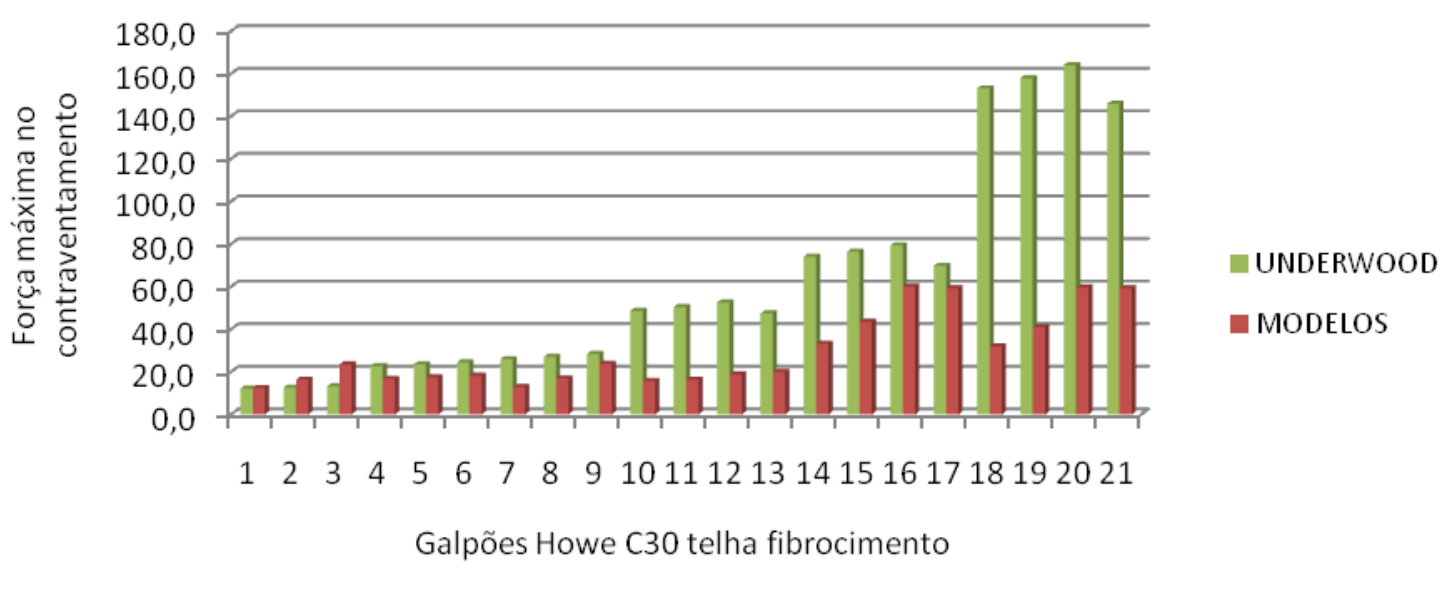

Gráfico 10 - Colunas comparativas do método SABS 0163 (HOWE C30)

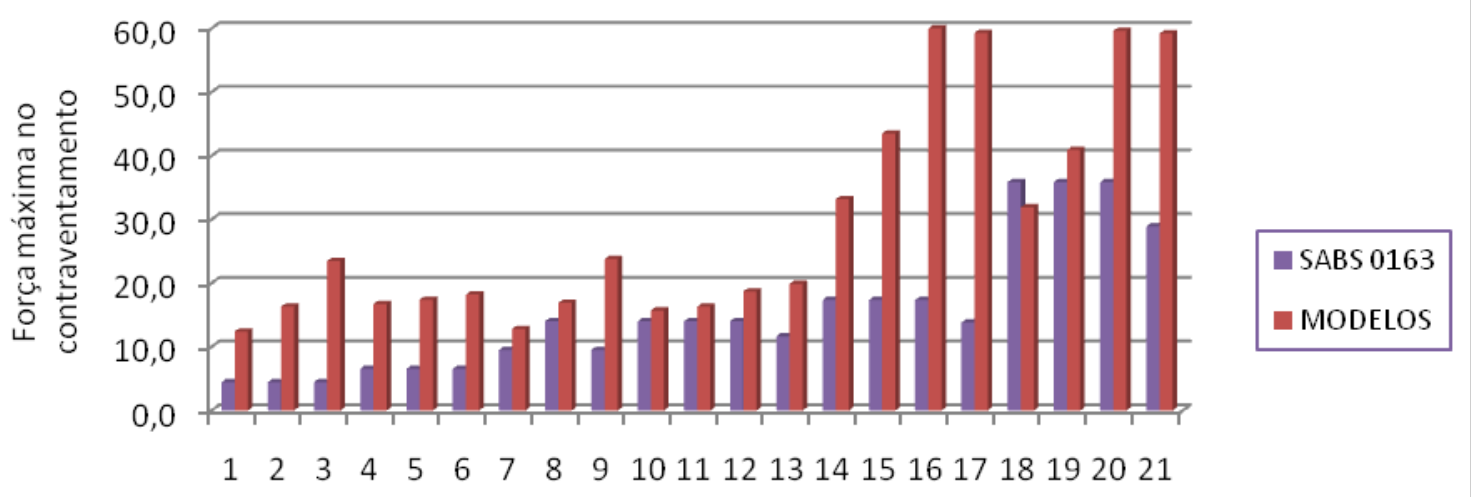

Galpões Howe C30 telha fibrocimento 


\subsection{Treliças PRATT C50 contraventamento de CABOS}

Tabela 5.9 - Comparativo entre o método da NBR 7190 (1997) e os modelos tridimensionais (PRATT C50)

\begin{tabular}{|c|c|c|c|c|c|c|c|c|c|c|c|c|}
\hline & & & & OME & TRIAS & DAS 1 & RELI & ÇAS & & & & \\
\hline $\mathrm{L}(\mathrm{v}$ & & 12 & 12 & 12 & 18 & 18 & 18 & 18 & 24 & 24 & 24 & 24 \\
\hline h (altura & entral) & 1,5 & 1,5 & 1,5 & 2,25 & 2,25 & 2,25 & 2,25 & 3 & 3 & 3 & 3 \\
\hline z (altura & pilar) & 3 & 4 & 6 & 3 & 4 & 6 & 6 & 3 & 4 & 6 & 6 \\
\hline e (dist. entr & treliças) & 3 & 3 & 3 & 3 & 3 & 3 & 4 & 3 & 3 & 3 & 4 \\
\hline & & & & MÉTO & DO NI & BR 719 & $0(199$ & & & & & \\
\hline Compressã & máx $(\mathrm{kN})$ & 82,4 & 85,8 & 82,2 & 157,3 & 161,6 & 162,6 & 198,2 & 233,4 & 240,0 & 250,4 & 300,9 \\
\hline & $F_{1 d}$ & 0,5 & 0,6 & 0,5 & 1,0 & 1,1 & 1,1 & 1,3 & 1,6 & 1,6 & 1,7 & 2,0 \\
\hline & $\mathrm{n}=7$ & 2,6 & 2,7 & 2,6 & 4,9 & 5,0 & 5,1 & - & - & - & - & - \\
\hline$F_{d}(k N)$ & $\mathrm{n}=11$ & - & - & - & - & - & - & 9,7 & - & - & - & 14,7 \\
\hline $29(\sin )$ & $\mathrm{n}=15$ & 5,5 & 5,7 & 5,5 & 10,5 & 10,8 & 10,8 & - & 15,6 & 16,0 & 16,7 & - \\
\hline & $\mathrm{n}=23$ & - & - & - & - & - & - & - & - & - & - & 30,8 \\
\hline & $\mathrm{n}=31$ & - & - & - & - & - & - & - & 32,2 & 33,1 & 34,5 & - \\
\hline & & & & MODI & ELOS & NUMÉ & RICO & & & & & \\
\hline Compressã & máx $(\mathrm{kN})$ & 94,0 & 97,7 & 102,3 & 153,0 & 158,8 & 166,1 & 216,6 & 246,0 & 254,0 & 263,2 & 316,3 \\
\hline & $\mathrm{n}=7$ & 12,4 & 16,3 & 23,5 & 9,7 & 12,4 & 15,4 & - & - & - & - & - \\
\hline & $\mathrm{n}=11$ & - & - & - & - & - & - & 18,5 & - & - & - & 59,3 \\
\hline $\mathbf{N}_{\mathbf{d}, \mathrm{t}}(\mathrm{kN})$ & $\mathrm{n}=15$ & 12,8 & 16,9 & 24,0 & 11,9 & 13,7 & 17,0 & - & 33,3 & 43,5 & 60,0 & - \\
\hline & $n=23$ & - & - & - & - & - & - & - & - & - & - & 59,2 \\
\hline & $\mathrm{n}=31$ & - & - & - & - & - & - & - & 32,1 & 41,0 & 59,6 & - \\
\hline & & & & MPAI & RATIV & O Gal & õo/Tr & eliça & & & & \\
\hline Compressã & máx $(\mathrm{kN})$ & 1,14 & 1,14 & 1,24 & 0,97 & 0,98 & 1,02 & 1,09 & 1,05 & 1,06 & 1,05 & 1,05 \\
\hline & $\mathrm{n}=7$ & 4,84 & 6,11 & 9,19 & 1,98 & 2,47 & 3,04 & - & - & - & - & - \\
\hline & $\mathrm{n}=11$ & - & - & - & - & - & - & 1,91 & - & - & - & 4,03 \\
\hline $\mathbf{N}_{\mathrm{d}, \mathbf{t}} / \mathbf{F}_{\mathbf{d}}$ & $\mathrm{n}=15$ & 2,33 & 2,95 & 4,38 & 1,13 & 1,27 & 1,57 & & 2,14 & 2,72 & 3,59 & - \\
\hline & $\mathrm{n}=23$ & - & - & - & - & - & - & - & - & - & - & 1,92 \\
\hline & $\mathrm{n}=31$ & - & - & - & - & - & - & - & 1,00 & 1,24 & 1,73 & - \\
\hline
\end{tabular}


Tabela 5.10 - Comparativo entre o método do EUROCODE 5 e os modelos tridimensionais (PRATT C50)

\begin{tabular}{|c|c|c|c|c|c|c|c|c|c|c|c|c|}
\hline & & & GE & OME & TRIAS & DAS 1 & RELI & ÇAS & & & & \\
\hline L (v & & 12 & 12 & 12 & 18 & 18 & 18 & 18 & 24 & 24 & 24 & 24 \\
\hline h (altura & entral) & 1,5 & 1,5 & 1,5 & 2,25 & 2,25 & 2,25 & 2,25 & 3 & 3 & 3 & 3 \\
\hline z (altura & pilar) & 3 & 4 & 6 & 3 & 4 & 6 & 6 & 3 & 4 & 6 & 6 \\
\hline e (dist. ent & treliças) & 3 & 3 & 3 & 3 & 3 & 3 & 4 & 3 & 3 & 3 & 4 \\
\hline & & & & MÉT & ODO E & UROC & ODE 5 & & & & & \\
\hline Compressã & máx $(\mathrm{kN})$ & 82,4 & 85,8 & 82,2 & 157,3 & 161,6 & 162,6 & 198,2 & 233,4 & 240,0 & 250,4 & $300, \mathrm{c}$ \\
\hline & $\mathrm{F}_{1 \mathrm{~d}}$ & 1,6 & 1,7 & 1,6 & 3,1 & 3,2 & 3,3 & 4,0 & 4,7 & 4,8 & 5,0 & 6,0 \\
\hline & $\mathrm{n}=7$ & 7,7 & 8,0 & 7,7 & 14,7 & 15,1 & 15,2 & - & - & - & - & - \\
\hline$F_{1}(k N)$ & $\mathrm{n}=11$ & - & - & - & - & - & - & 29,1 & - & - & - & 44,1 \\
\hline $\log (\sin )$ & $\mathrm{n}=15$ & 16,5 & 17,2 & 16,4 & 31,5 & 32,3 & 32,5 & & 46,7 & 48,0 & 50,1 & - \\
\hline & $\mathrm{n}=23$ & - & - & - & - & - & - & - & - & - & - & 92,3 \\
\hline & $\mathrm{n}=31$ & - & - & - & - & - & - & - & 96,5 & 99,2 & 103,5 & - \\
\hline & & & & MOD & ELOS & NUMÉ & RICOS & & & & & \\
\hline Compressấ & máx $(\mathrm{kN})$ & 94,0 & 97,7 & 102,3 & 153,0 & 158,8 & 166,1 & 216,6 & 246,0 & 254,0 & 263,2 & 316,3 \\
\hline & $\mathrm{n}=7$ & 12,4 & 16,3 & 23,5 & 9,7 & 12,4 & 15,4 & - & - & - & - & - \\
\hline & $\mathrm{n}=11$ & - & - & - & - & - & - & 18,5 & - & - & - & 59,3 \\
\hline $\begin{array}{c}\mathbf{N}_{\mathrm{d}, \mathrm{t}}(\mathrm{KIV}) \\
\text { Tercas }\end{array}$ & $\mathrm{n}=15$ & 12,8 & 16,9 & 24,0 & 11,9 & 13,7 & 17,0 & & 33,3 & 43,5 & 60,0 & - \\
\hline & $\mathrm{n}=23$ & - & - & - & - & - & - & - & - & - & - & 59,2 \\
\hline & $\mathrm{n}=31$ & - & - & - & - & - & - & - & 32,1 & 41,0 & 59,6 & - \\
\hline & & & $\mathrm{CC}$ & MPAI & RATIV & O Gal & pão/Tr & eliça & & & & \\
\hline Compressã & máx $(\mathrm{kN})$ & 1,14 & 1,14 & 1,24 & 0,97 & 0,98 & 1,02 & 1,09 & 1,05 & 1,06 & 1,05 & 1,05 \\
\hline & $\mathrm{n}=7$ & 1,61 & 2,04 & 3,06 & 0,66 & 0,82 & 1,01 & - & - & - & - & - \\
\hline & $\mathrm{n}=11$ & - & - & - & - & - & - & 0,64 & - & - & - & 1,34 \\
\hline $\mathbf{N}_{\mathrm{d}, \mathrm{t}} / \mathbf{F}_{\mathbf{d}}$ & $\mathrm{n}=15$ & 0,78 & 0,98 & 1,46 & 0,38 & 0,42 & 0,52 & & 0,71 & 0,91 & 1,20 & - \\
\hline & $\mathrm{n}=23$ & - & - & - & - & - & - & - & - & - & - & 0,64 \\
\hline & $n=31$ & - & - & - & - & - & - & & 0,33 & 0,41 & 0,58 & - \\
\hline
\end{tabular}


Tabela 5.11 - Comparativo entre o método de UNDERWOOD (2000) e os modelos tridimensionais (PRATT C50)

\begin{tabular}{|c|c|c|c|c|c|c|c|c|c|c|c|c|}
\hline \multicolumn{13}{|c|}{ GEOMETRIAS DAS TRELIÇAS } \\
\hline \multicolumn{2}{|c|}{ L (vão) } & 12 & 12 & 12 & 18 & 18 & 18 & 18 & 24 & 24 & 24 & 24 \\
\hline \multicolumn{2}{|c|}{ h (altura central) } & 1,5 & 1,5 & 1,5 & 2,25 & 2,25 & 2,25 & 2,25 & 3 & 3 & 3 & 3 \\
\hline \multicolumn{2}{|c|}{ z (altura do pilar) } & 3 & 4 & 6 & 3 & 4 & 6 & 6 & 3 & 4 & 6 & 6 \\
\hline \multicolumn{2}{|c|}{ e (dist. entre treliças) } & 3 & 3 & 3 & 3 & 3 & 3 & 4 & 3 & 3 & 3 & 4 \\
\hline \multicolumn{13}{|c|}{ MÉTODO UNDERWOOD (2000) } \\
\hline \multicolumn{2}{|c|}{ Compressão $_{\text {máx }}(\mathrm{kN})$} & 82,4 & 85,8 & 82,2 & 157,3 & 161,6 & 162,6 & 198,2 & 233,4 & 240,0 & 250,4 & 300,9 \\
\hline \multirow{6}{*}{$F_{d}(k N)$} & $F_{1 \mathrm{~d}}$ & 2,6 & 2,7 & 2,6 & 4,9 & 5,1 & 5,1 & 6,2 & 7,3 & 7,5 & 7,8 & 9,4 \\
\hline & $\mathrm{n}=7$ & 12,0 & 12,5 & 12,0 & 22,9 & 23,6 & 23,7 & - & - & - & - & - \\
\hline & $\mathrm{n}=11$ & - & - & - & - & - & - & 45,4 & - & - & - & 69,0 \\
\hline & $\mathrm{n}=15$ & 25,8 & 26,8 & 25,7 & 49,2 & 50,5 & 50,8 & - & 72,9 & 75,0 & 78,3 & - \\
\hline & $\mathrm{n}=23$ & - & - & - & - & - & - & - & - & - & - & 144,2 \\
\hline & $\mathrm{n}=31$ & - & - & - & - & - & - & - & 150,7 & 155,0 & 161,7 & - \\
\hline \multicolumn{13}{|c|}{ MODELOS NUMÉRICOS } \\
\hline \multicolumn{2}{|c|}{ Compressão máx $_{(\mathrm{kN})}$} & 94,0 & 97,7 & 102,3 & 153,0 & 158,8 & 166,1 & 216,6 & 246,0 & 254,0 & 263,2 & 316,3 \\
\hline \multirow{5}{*}{$\begin{array}{c}\mathbf{N}_{\mathbf{d}, t}(\mathbf{k N}) \\
\text { Terças }\end{array}$} & $\mathrm{n}=7$ & 12,4 & 16,3 & 23,5 & 9,7 & 12,4 & 15,4 & - & - & - & - & - \\
\hline & $\mathrm{n}=11$ & - & - & - & - & - & - & 18,5 & - & - & - & 59,3 \\
\hline & $\mathrm{n}=15$ & 12,8 & 16,9 & 24,0 & 11,9 & 13,7 & 17,0 & - & 33,3 & 43,5 & 60,0 & - \\
\hline & $\mathrm{n}=23$ & - & - & - & - & - & - & - & - & - & - & 59,2 \\
\hline & $\mathrm{n}=31$ & - & - & - & - & - & - & - & 32,1 & 41,0 & 59,6 & - \\
\hline \multicolumn{13}{|c|}{ COMPARATIVO Galpão/Treliça } \\
\hline \multicolumn{2}{|c|}{ Compressão máx $(\mathrm{kN})$} & 1,14 & 1,14 & 1,24 & 0,97 & 0,98 & 1,02 & 1,09 & 1,05 & 1,06 & 1,05 & 1,05 \\
\hline \multirow{5}{*}{$\mathbf{N}_{\mathbf{d}, \mathrm{t}} / \mathbf{F}_{\mathbf{d}}$} & $\mathrm{n}=7$ & 1,03 & 1,30 & 1,96 & 0,42 & 0,53 & 0,65 & - & - & - & - & - \\
\hline & $\mathrm{n}=11$ & - & - & - & - & - & - & 0,41 & - & - & - & $\mathbf{0 , 8 6}$ \\
\hline & $\mathrm{n}=15$ & 0,50 & 0,63 & 0,93 & 0,24 & 0,27 & 0,33 & - & 0,46 & 0,58 & 0,77 & \\
\hline & $\mathrm{n}=23$ & - & - & - & - & - & - & - & - & - & - & 0,41 \\
\hline & $\mathrm{n}=31$ & - & - & - & - & - & - & - & 0,21 & 0,26 & 0,37 & - \\
\hline
\end{tabular}


Tabela 5.12 - Comparativo entre o método do SABS 0163 e os modelos tridimensionais (PRATT C50)

\begin{tabular}{|c|c|c|c|c|c|c|c|c|c|c|c|c|}
\hline & & & & OMET & [RIAS & DAS T & TRELI & ÇAS & & & & \\
\hline $\mathrm{L}(\mathrm{v}$ & & 12 & 12 & 12 & 18 & 18 & 18 & 18 & 24 & 24 & 24 & 24 \\
\hline h (altura & entral) & 1,5 & 1,5 & 1,5 & 2,25 & 2,25 & 2,25 & 2,25 & 3 & 3 & 3 & 3 \\
\hline $\mathrm{z}$ (altura & pilar) & 3 & 4 & 6 & 3 & 4 & 6 & 6 & 3 & 4 & 6 & 6 \\
\hline e (dist. entr & treliças) & 3 & 3 & 3 & 3 & 3 & 3 & 4 & 3 & 3 & 3 & 4 \\
\hline & & & & MÉ & TODO & SABS & 0163 & & & & & \\
\hline Compressã & erm $(k N)$ & & 39,5 & & & 77,4 & & 86,2 & & 135,2 & & 147,3 \\
\hline & $\mathrm{F}_{1 \mathrm{~d}}$ & & 1,0 & & & 1,3 & & 1,4 & & 1,7 & & 1,8 \\
\hline & $\mathrm{n}=7$ & 4,6 & 4,6 & 4,6 & 6,0 & 6,0 & 6,0 & - & - & - & - & - \\
\hline $\mathrm{F}_{\mathrm{A}}(\mathrm{kN})$ & $\mathrm{n}=11$ & - & - & - & - & - & - & 10,5 & - & - & - & 13,5 \\
\hline$F d(k i v)$ & $\mathrm{n}=15$ & 9,9 & 12,9 & 9,9 & 12,9 & 12,9 & 12,9 & - & 16,9 & 16,9 & 16,9 & \\
\hline & $n=23$ & - & - & - & - & - & - & - & - & - & - & 28,2 \\
\hline & $\mathrm{n}=31$ & - & - & - & - & - & - & - & 34,9 & 34,9 & 34,9 & - \\
\hline & & & & MODI & ELOS I & NUMÉ & RICOS & & & & & \\
\hline Compressã & máx $(\mathrm{kN})$ & 94,0 & 97,7 & 102,3 & 153,0 & 158,8 & 166,1 & 216,6 & 246,0 & 254,0 & 263,2 & 316,3 \\
\hline & $\mathrm{n}=7$ & 12,4 & 16,3 & 23,5 & 9,7 & 12,4 & 15,4 & - & - & - & & - \\
\hline & $\mathrm{n}=11$ & - & - & - & - & - & - & 18,5 & - & - & - & 59,3 \\
\hline $\begin{array}{c}\mathbf{N}_{\mathrm{d}, \mathrm{t}}(\mathrm{kN}) \\
\text { Tercas }\end{array}$ & $\mathrm{n}=15$ & 12,8 & 16,9 & 24,0 & 11,9 & 13,7 & 17,0 & - & 33,3 & 43,5 & 60,0 & - \\
\hline & $\mathrm{n}=23$ & - & - & - & - & - & - & - & - & - & - & 59,2 \\
\hline & $\mathrm{n}=31$ & - & - & - & - & - & - & - & 32,1 & 41,0 & 59,6 & - \\
\hline & & & & MPAF & RATIV & O Galp & pão/Tr & eliça & & & & \\
\hline & $\mathrm{n}=7$ & 2,69 & 3,54 & 5,10 & 1,61 & 2,06 & 2,56 & - & - & - & - & - \\
\hline & $\mathrm{n}=11$ & - & - & - & - & - & - & 1,76 & - & - & - & 4,39 \\
\hline $\mathbf{N}_{\mathrm{d}, \mathrm{t}} / \mathbf{F}_{\mathbf{d}}$ & $\mathrm{n}=15$ & 1,30 & 1,31 & 2,43 & 0,92 & 1,06 & 1,32 & - & 1,97 & 2,57 & 3,55 & \\
\hline & $n=23$ & - & - & - & - & - & - & - & - & - & - & 2,10 \\
\hline & $\mathrm{n}=31$ & - & - & - & - & - & - & - & 0,92 & 1,17 & 1,71 & - \\
\hline
\end{tabular}


Gráfico 11 - Linhas comparativas dos métodos (PRATT C50 cabos)

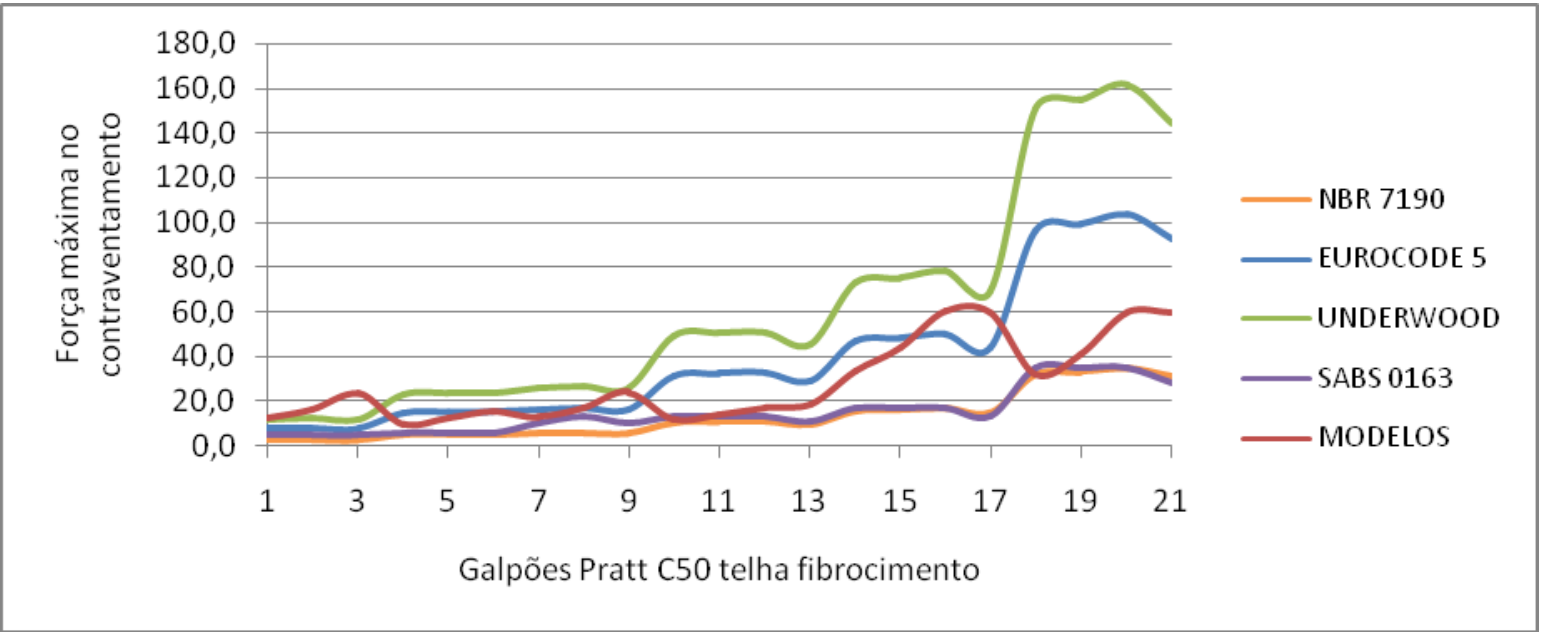

Gráfico 12 - Colunas comparativas do método NBR 7190 (PRATT C50 cabos)

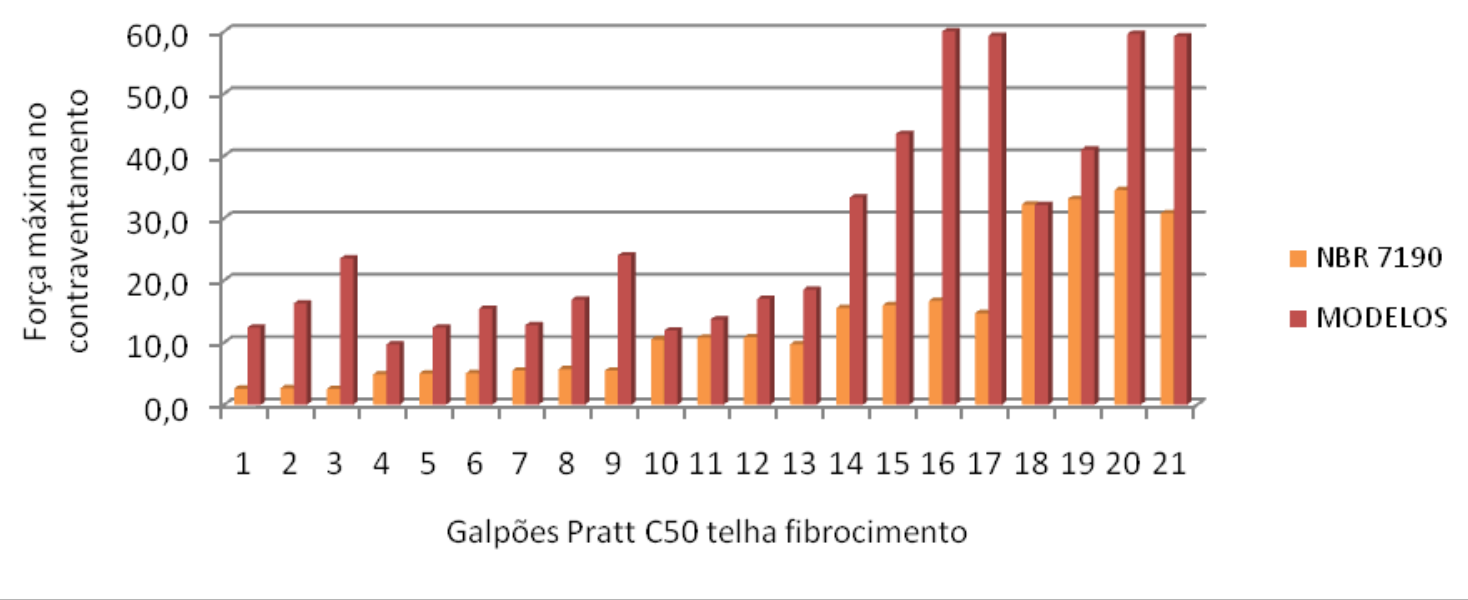

Gráfico 13 - Colunas comparativas do método EUROCODE 5 (PRATT C50 cabos)

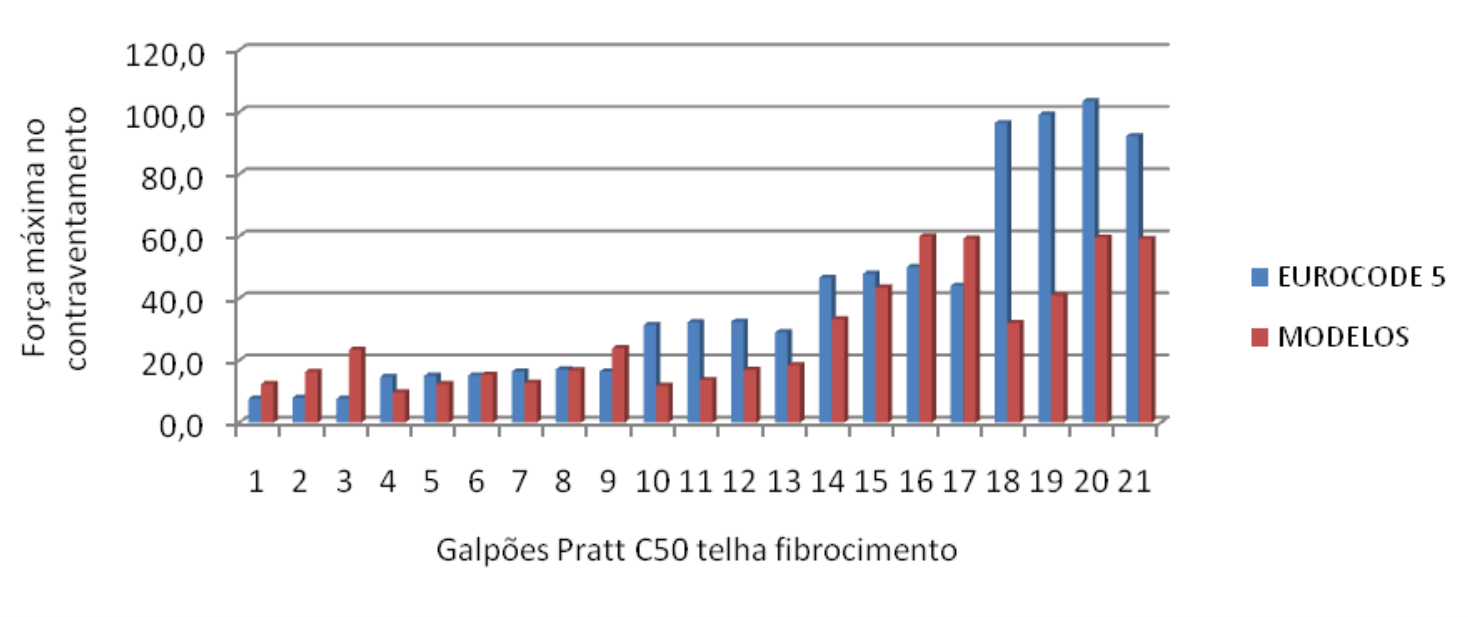


Gráfico 14 - Colunas comparativas do método UNDERWOOD (PRATT C50 cabos)

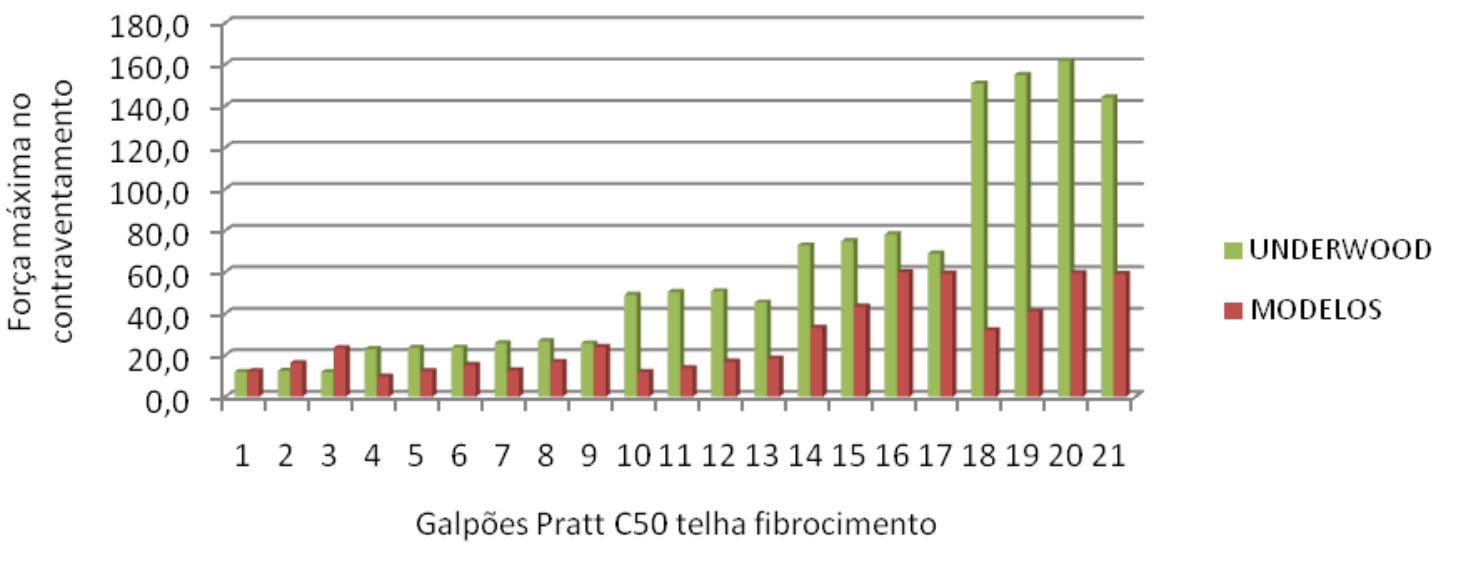

Gráfico 15 - Colunas comparativas do método SABS 0163 (PRATT C50 cabos)

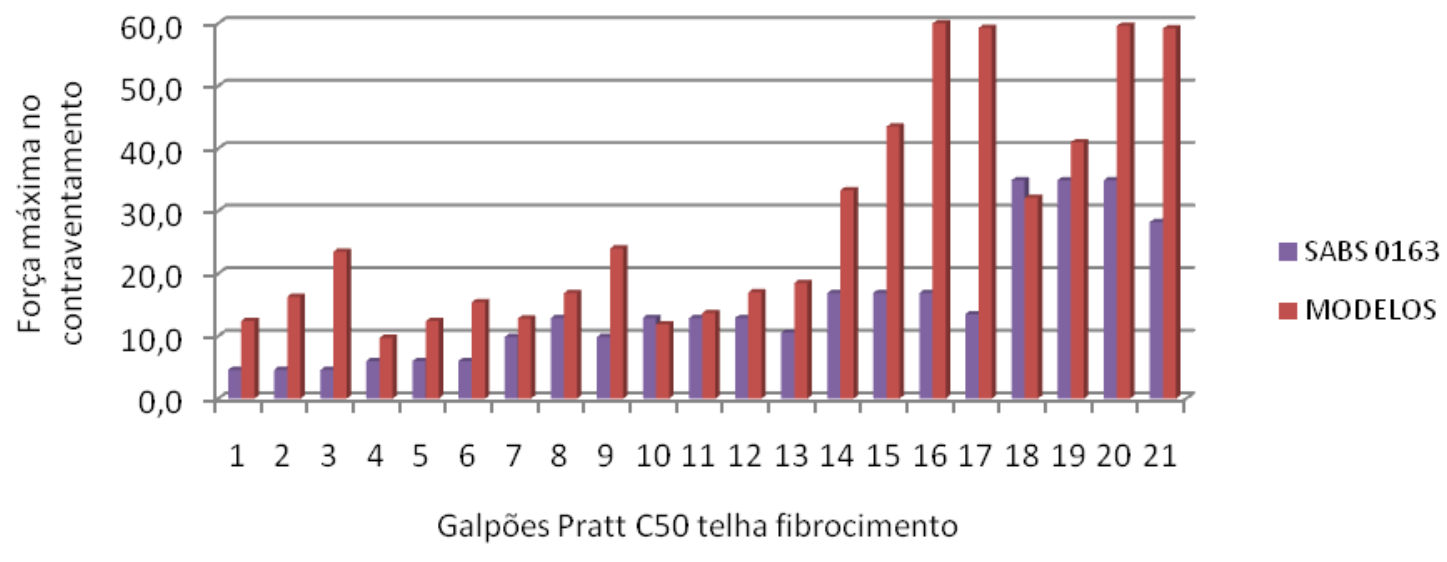




\subsection{Treliças PRATT C50 contraventamento de MADEIRA}

Tabela 5.13 - Comparativo entre o método da NBR 7190 (1997) e os modelos tridimensionais (PRATT C50 madeira)

\begin{tabular}{|c|c|c|c|c|c|c|c|c|c|c|c|c|}
\hline \multicolumn{13}{|c|}{ GEOMETRIAS DAS TRELIÇAS } \\
\hline \multicolumn{2}{|c|}{ L (vão) } & 12 & 12 & 12 & 18 & 18 & 18 & 18 & 24 & 24 & 24 & 24 \\
\hline \multicolumn{2}{|c|}{ h (altura central) } & 1,5 & 1,5 & 1,5 & 2,25 & 2,25 & 2,25 & 2,25 & 3 & 3 & 3 & 3 \\
\hline \multicolumn{2}{|c|}{ z (altura do pilar) } & 3 & 4 & 6 & 3 & 4 & 6 & 6 & 3 & 4 & 6 & 6 \\
\hline \multicolumn{2}{|c|}{ e (dist. entre treliças) } & 3 & 3 & 3 & 3 & 3 & 3 & 4 & 3 & 3 & 3 & 4 \\
\hline \multicolumn{13}{|c|}{ MÉTODO NBR 7190 (1997) } \\
\hline \multicolumn{2}{|c|}{ Compressão $_{\text {máx }}(\mathrm{kN})$} & 82,4 & 85,8 & 82,2 & 157,3 & 161,6 & 162,6 & 198,2 & 233,4 & 240,0 & 250,4 & 300,9 \\
\hline \multirow{6}{*}{$F_{d}(k N)$} & $\mathrm{F}_{1 \mathrm{~d}}$ & 0,5 & 0,6 & 0,5 & 1,0 & 1,1 & 1,1 & 1,3 & 1,6 & 1,6 & 1,7 & 2,0 \\
\hline & $\mathrm{n}=7$ & 2,6 & 2,7 & 2,6 & 4,9 & 5,0 & 5,1 & - & - & - & - & - \\
\hline & $\mathrm{n}=11$ & - & - & - & - & - & - & 9,7 & - & - & - & 14,7 \\
\hline & $\mathrm{n}=15$ & 5,5 & 5,7 & 5,5 & 10,5 & 10,8 & 10,8 & - & 15,6 & 16,0 & 16,7 & - \\
\hline & $\mathrm{n}=23$ & - & - & - & - & - & - & - & - & - & - & 30,8 \\
\hline & $\mathrm{n}=31$ & - & - & - & - & - & - & - & 32,2 & 33,1 & 34,5 & - \\
\hline \multicolumn{13}{|c|}{ MODELOS NUMÉRICOS } \\
\hline \multicolumn{2}{|c|}{ Compressão máx $_{\text {(kN) }}$} & 101,0 & 104,5 & 107,9 & 164,2 & 169,9 & 177,1 & 233,8 & 263,5 & 271,3 & 280,2 & 343,2 \\
\hline \multirow{5}{*}{$\begin{array}{c}\mathbf{N}_{\mathrm{d}, \mathrm{t}}(\mathbf{k N}) \\
\text { Terças }\end{array}$} & $\mathrm{n}=7$ & 12,8 & 13,3 & 15,8 & 12,4 & 13,5 & 16,1 & - & & - & - & - \\
\hline & $\mathrm{n}=11$ & - & - & - & - & - & - & 13,7 & - & - & - & 35,7 \\
\hline & $\mathrm{n}=15$ & 12,5 & 12,9 & 13,4 & 17,0 & 17,4 & 18,0 & - & 24,2 & 29,3 & 37,3 & - \\
\hline & $\mathrm{n}=23$ & - & - & - & - & - & - & - & - & - & - & 36,9 \\
\hline & $\mathrm{n}=31$ & - & - & & & - & - & - & 24,3 & 28,8 & 38,5 & - \\
\hline \multicolumn{13}{|c|}{ COMPARATIVO Galpão/Treliça } \\
\hline \multicolumn{2}{|c|}{ Compressão $_{\text {máx }}(\mathrm{kN})$} & 1,23 & 1,22 & 1,31 & 1,04 & 1,05 & 1,09 & 1,18 & 1,13 & 1,13 & 1,12 & 1,14 \\
\hline \multirow{5}{*}{$\mathbf{N}_{\mathbf{d}, \mathrm{t}} / \mathbf{F}_{\mathbf{d}}$} & $\mathrm{n}=7$ & 4,99 & 4,98 & 6,18 & 2,53 & 2,69 & 3,18 & - & & - & - & - \\
\hline & $\mathrm{n}=11$ & - & - & - & - & - & - & 1,41 & - & - & - & 2,43 \\
\hline & $\mathrm{n}=15$ & 2,28 & 2,26 & 2,45 & 1,62 & 1,62 & 1,66 & - & 1,56 & 1,83 & 2,23 & - \\
\hline & $\mathrm{n}=23$ & - & - & - & - & - & - & - & - & - & - & 1,20 \\
\hline & $\mathrm{n}=31$ & - & - & - & - & - & - & - & 0,76 & 0,87 & 1,12 & - \\
\hline
\end{tabular}


Tabela 5.14 - Comparativo entre o método do EUROCODE 5 e os modelos tridimensionais (PRATT C50 madeira)

\begin{tabular}{|c|c|c|c|c|c|c|c|c|c|c|c|c|}
\hline \multicolumn{13}{|c|}{ GEOMETRIAS DAS TRELIÇAS } \\
\hline \multicolumn{2}{|c|}{ L (vão) } & 12 & 12 & 12 & 18 & 18 & 18 & 18 & 24 & 24 & 24 & 24 \\
\hline \multicolumn{2}{|c|}{ h (altura central) } & 1,5 & 1,5 & 1,5 & 2,25 & 2,25 & 2,25 & 2,25 & 3 & 3 & 3 & 3 \\
\hline \multicolumn{2}{|c|}{ z (altura do pilar) } & 3 & 4 & 6 & 3 & 4 & 6 & 6 & 3 & 4 & 6 & 6 \\
\hline \multicolumn{2}{|c|}{ e (dist. entre treliças) } & 3 & 3 & 3 & 3 & 3 & 3 & 4 & 3 & 3 & 3 & 4 \\
\hline \multicolumn{13}{|c|}{ MÉTODO EUROCODE 5} \\
\hline \multicolumn{2}{|c|}{ Compressão $_{\text {máx }}(\mathrm{kN})$} & 82,4 & 85,8 & 82,2 & 157,3 & 161,6 & 162,6 & 198,2 & 233,4 & 240,0 & 250,4 & 300,9 \\
\hline \multirow{6}{*}{$F_{d}(k N)$} & $\mathrm{F}_{1 \mathrm{~d}}$ & 1,6 & 1,7 & 1,6 & 3,1 & 3,2 & 3,3 & 4,0 & 4,7 & 4,8 & 5,0 & 6,0 \\
\hline & $\mathrm{n}=7$ & 7,7 & 8,0 & 7,7 & 14,7 & 15,1 & 15,2 & - & - & - & - & - \\
\hline & $\mathrm{n}=11$ & - & - & - & - & - & - & 29,1 & - & - & - & 44,1 \\
\hline & $\mathrm{n}=15$ & 16,5 & 17,2 & 16,4 & 31,5 & 32,3 & 32,5 & - & 46,7 & 48,0 & 50,1 & - \\
\hline & $\mathrm{n}=23$ & - & - & - & - & - & - & - & - & - & - & 92,3 \\
\hline & $\mathrm{n}=31$ & - & - & - & - & & - & - & 96,5 & 99,2 & 103,5 & - \\
\hline \multicolumn{13}{|c|}{ MODELOS NUMÉRICOS } \\
\hline \multicolumn{2}{|c|}{ Compressãomáx $(\mathrm{kN})$} & 101,0 & 104,5 & 107,9 & 164,2 & 169,9 & 177,1 & 233,8 & 263,5 & 271,3 & 280,2 & 343,2 \\
\hline \multirow{5}{*}{$\begin{array}{c}\mathbf{N}_{\mathrm{d}, \mathrm{t}}(\mathbf{k N}) \\
\text { Terças }\end{array}$} & $\mathrm{n}=7$ & 12,8 & 13,3 & 15,8 & 12,4 & 13,5 & 16,1 & - & - & - & - & - \\
\hline & $\mathrm{n}=11$ & - & - & - & - & - & - & 13,7 & - & - & - & 35,7 \\
\hline & $\mathrm{n}=15$ & 12,5 & 12,9 & 13,4 & 17,0 & 17,4 & 18,0 & - & 24,2 & 29,3 & 37,3 & - \\
\hline & $\mathrm{n}=23$ & - & - & - & - & - & - & - & - & - & - & 36,9 \\
\hline & $\mathrm{n}=31$ & - & - & - & - & - & - & - & 24,3 & 28,8 & 38,5 & - \\
\hline \multicolumn{13}{|c|}{ COMPARATIVO Galpão/Treliça } \\
\hline \multicolumn{2}{|c|}{ Compressão máx $(\mathrm{kN})$} & 1,23 & 1,22 & 1,31 & 1,04 & 1,05 & 1,09 & 1,18 & 1,13 & 1,13 & 1,12 & 1,14 \\
\hline \multirow{5}{*}{$\mathbf{N}_{\mathrm{d}, \mathrm{t}} / \mathbf{F}_{\mathrm{d}}$} & $\mathrm{n}=7$ & 1,66 & 1,66 & 2,06 & $\mathbf{0 , 8 4}$ & 0,90 & 1,06 & - & - & - & - & - \\
\hline & $\mathrm{n}=11$ & - & - & - & - & - & - & 0,47 & - & - & - & $\mathbf{0 , 8 1}$ \\
\hline & $\mathrm{n}=15$ & $\mathbf{0 , 7 6}$ & 0,75 & 0,82 & 0,54 & 0,54 & 0,55 & - & 0,52 & 0,61 & 0,74 & - \\
\hline & $\mathrm{n}=23$ & - & - & - & - & - & - & - & - & - & - & 0,40 \\
\hline & $\mathrm{n}=31$ & - & - & - & - & - & - & - & 0,25 & 0,29 & 0,37 & - \\
\hline
\end{tabular}


Tabela 5.15 - Comparativo entre o método de UNDERWOOD (2000) e os modelos tridimensionais (PRATT C50 madeira)

\begin{tabular}{|c|c|c|c|c|c|c|c|c|c|c|c|c|}
\hline \multicolumn{13}{|c|}{ GEOMETRIAS DAS TRELIÇAS } \\
\hline \multicolumn{2}{|c|}{ L (vão) } & 12 & 12 & 12 & 18 & 18 & 18 & 18 & 24 & 24 & 24 & 24 \\
\hline \multicolumn{2}{|c|}{ h (altura central) } & 1,5 & 1,5 & 1,5 & 2,25 & 2,25 & 2,25 & 2,25 & 3 & 3 & 3 & 3 \\
\hline \multicolumn{2}{|c|}{ z (altura do pilar) } & 3 & 4 & 6 & 3 & 4 & 6 & 6 & 3 & 4 & 6 & 6 \\
\hline \multicolumn{2}{|c|}{ e (dist. entre treliças) } & 3 & 3 & 3 & 3 & 3 & 3 & 4 & 3 & 3 & 3 & 4 \\
\hline \multicolumn{13}{|c|}{ MÉTODO UNDERWOOD (2000) } \\
\hline \multicolumn{2}{|c|}{ Compressão ${ }_{\text {máx }}(\mathrm{kN})$} & 82,4 & 85,8 & 82,2 & 157,3 & 161,6 & 162,6 & 198,2 & 233,4 & 240,0 & 250,4 & 300,9 \\
\hline \multirow{6}{*}{$F_{d}(k N)$} & $F_{1 d}$ & 2,6 & 2,7 & 2,6 & 4,9 & 5,1 & 5,1 & 6,2 & 7,3 & 7,5 & 7,8 & 9,4 \\
\hline & $\mathrm{n}=7$ & 12,0 & 12,5 & 12,0 & 22,9 & 23,6 & 23,7 & - & & - & - & - \\
\hline & $\mathrm{n}=11$ & - & - & - & - & - & - & 45,4 & - & - & - & 69,0 \\
\hline & $\mathrm{n}=15$ & 25,8 & 26,8 & 25,7 & 49,2 & 50,5 & 50,8 & - & 72,9 & 75,0 & 78,3 & - \\
\hline & $\mathrm{n}=23$ & - & - & - & - & - & - & - & - & - & - & 144,2 \\
\hline & $\mathrm{n}=31$ & - & - & & - & - & - & - & 150,7 & 155,0 & 161,7 & - \\
\hline \multicolumn{13}{|c|}{ MODELOS NUMÉRICOS } \\
\hline \multicolumn{2}{|c|}{ Compressãomáx $_{\text {mN) }}$} & 101,0 & 104,5 & 107,9 & 164,2 & 169,9 & 177,1 & 233,8 & 263,5 & 271,3 & 280,2 & 343,2 \\
\hline \multirow{5}{*}{$\begin{array}{c}\mathbf{N}_{\mathrm{d}, \mathrm{t}}(\mathbf{k N}) \\
\text { Terças }\end{array}$} & $\mathrm{n}=7$ & 12,8 & 13,3 & 15,8 & 12,4 & 13,5 & 16,1 & - & - & - & - & - \\
\hline & $\mathrm{n}=11$ & - & - & - & - & - & - & 13,7 & - & - & - & 35,7 \\
\hline & $\mathrm{n}=15$ & 12,5 & 12,9 & 13,4 & 17,0 & 17,4 & 18,0 & - & 24,2 & 29,3 & 37,3 & - \\
\hline & $\mathrm{n}=23$ & - & - & - & - & - & - & - & - & - & - & 36,9 \\
\hline & $\mathrm{n}=31$ & - & - & - & - & - & - & - & 24,3 & 28,8 & 38,5 & - \\
\hline \multicolumn{13}{|c|}{ COMPARATIVO Galpão/Treliça } \\
\hline \multicolumn{2}{|c|}{ Compressãomáx $_{\text {man) }}$} & 1,23 & 1,22 & 1,31 & 1,04 & 1,05 & 1,09 & 1,18 & 1,13 & 1,13 & 1,12 & 1,14 \\
\hline \multirow{5}{*}{$\mathbf{N}_{\mathrm{d}, \mathrm{t}} / \mathbf{F}_{\mathbf{d}}$} & $\mathrm{n}=7$ & 1,07 & 1,06 & 1,32 & 0,54 & 0,57 & 0,68 & - & - & - & - & - \\
\hline & $\mathrm{n}=11$ & - & - & - & - & - & - & $\mathbf{0 , 3 0}$ & - & - & - & 0,52 \\
\hline & $\mathrm{n}=15$ & 0,49 & 0,48 & 0,52 & 0,35 & 0,34 & 0,35 & - & 0,33 & 0,39 & 0,48 & - \\
\hline & $\mathrm{n}=23$ & - & - & - & - & - & - & - & - & - & - & 0,26 \\
\hline & $\mathrm{n}=31$ & - & - & - & - & - & - & - & 0,16 & 0,19 & 0,24 & - \\
\hline
\end{tabular}


Tabela 5.16 - Comparativo entre o método do SABS 0163 e os modelos tridimensionais (PRATT C50 madeira)

\begin{tabular}{|c|c|c|c|c|c|c|c|c|c|c|c|c|}
\hline \multicolumn{13}{|c|}{ GEOMETRIAS DAS TRELIÇAS } \\
\hline \multicolumn{2}{|c|}{ L (vão) } & 12 & 12 & 12 & 18 & 18 & 18 & 18 & 24 & 24 & 24 & 24 \\
\hline \multicolumn{2}{|c|}{ h (altura central) } & 1,5 & 1,5 & 1,5 & 2,25 & 2,25 & 2,25 & 2,25 & 3 & 3 & 3 & 3 \\
\hline \multicolumn{2}{|c|}{ z (altura do pilar) } & 3 & 4 & 6 & 3 & 4 & 6 & 6 & 3 & 4 & 6 & 6 \\
\hline \multicolumn{2}{|c|}{ e (dist. entre treliças) } & 3 & 3 & 3 & 3 & 3 & 3 & 4 & 3 & 3 & 3 & 4 \\
\hline \multicolumn{13}{|c|}{ MÉTODO SABS 0163} \\
\hline \multicolumn{2}{|c|}{ Compressão $_{\text {perm }}(\mathrm{kN})$} & \multicolumn{3}{|c|}{39,5} & \multicolumn{3}{|c|}{77,4} & 86,2 & \multicolumn{3}{|c|}{135,2} & 147,3 \\
\hline \multirow{6}{*}{$F_{d}(\mathbf{k N})$} & $\mathrm{F}_{1 \mathrm{~d}}$ & \multicolumn{3}{|c|}{1,0} & \multicolumn{3}{|c|}{1,3} & 1,4 & \multicolumn{3}{|c|}{1,7} & 1,8 \\
\hline & $\mathrm{n}=7$ & 4,6 & 4,6 & 4,6 & 6,0 & 6,0 & 6,0 & - & - & - & - & - \\
\hline & $\mathrm{n}=11$ & - & - & - & - & - & - & 10,5 & - & - & - & 13,5 \\
\hline & $\mathrm{n}=15$ & 9,9 & 12,9 & 9,9 & 12,9 & 12,9 & 12,9 & - & 16,9 & 16,9 & 16,9 & - \\
\hline & $\mathrm{n}=23$ & - & - & - & - & - & - & - & - & - & - & 28,2 \\
\hline & $\mathrm{n}=31$ & - & - & - & - & - & - & - & 34,9 & 34,9 & 34,9 & - \\
\hline \multicolumn{13}{|c|}{ MODELOS NUMÉRICOS } \\
\hline \multicolumn{2}{|c|}{ Compressão máx $(\mathrm{kN})$} & 101,0 & 104,5 & 107,9 & 164,2 & 169,9 & 177,1 & 233,8 & 263,5 & 271,3 & 280,2 & 343,2 \\
\hline \multirow{5}{*}{$\begin{array}{c}\mathbf{N}_{\mathrm{d}, \mathrm{t}}(\mathbf{k N}) \\
\text { Terças }\end{array}$} & $\mathrm{n}=7$ & 12,8 & 13,3 & 15,8 & 12,4 & 13,5 & 16,1 & - & - & - & - & - \\
\hline & $\mathrm{n}=11$ & - & - & - & - & - & - & 13,7 & - & - & - & 35,7 \\
\hline & $\mathrm{n}=15$ & 12,5 & 12,9 & 13,4 & 17,0 & 17,4 & 18,0 & - & 24,2 & 29,3 & 37,3 & - \\
\hline & $\mathrm{n}=23$ & - & - & - & - & - & - & - & - & - & - & 36,9 \\
\hline & $\mathrm{n}=31$ & - & - & - & - & - & - & - & 24,3 & 28,8 & 38,5 & - \\
\hline \multicolumn{13}{|c|}{ COMPARATIVO Galpão/Treliça } \\
\hline \multirow{5}{*}{$\mathbf{N}_{\mathrm{d}, \mathrm{t}} / \mathbf{F}_{\mathrm{d}}$} & $\mathrm{n}=7$ & 2,78 & 2,89 & 3,43 & 2,06 & 2,24 & 2,67 & - & - & - & - & - \\
\hline & $\mathrm{n}=11$ & - & - & - & - & - & - & 1,30 & - & - & - & 2,64 \\
\hline & $\mathrm{n}=15$ & 1,27 & 1,00 & 1,36 & 1,32 & 1,35 & 1,40 & - & 1,43 & 1,73 & 2,21 & - \\
\hline & $\mathrm{n}=23$ & - & - & - & - & - & - & - & - & - & - & 1,31 \\
\hline & $\mathrm{n}=31$ & - & - & - & - & - & - & - & 0,70 & 0,82 & 1,10 & - \\
\hline
\end{tabular}


Gráfico 16 - Linhas comparativas dos métodos (PRATT C50 madeira)

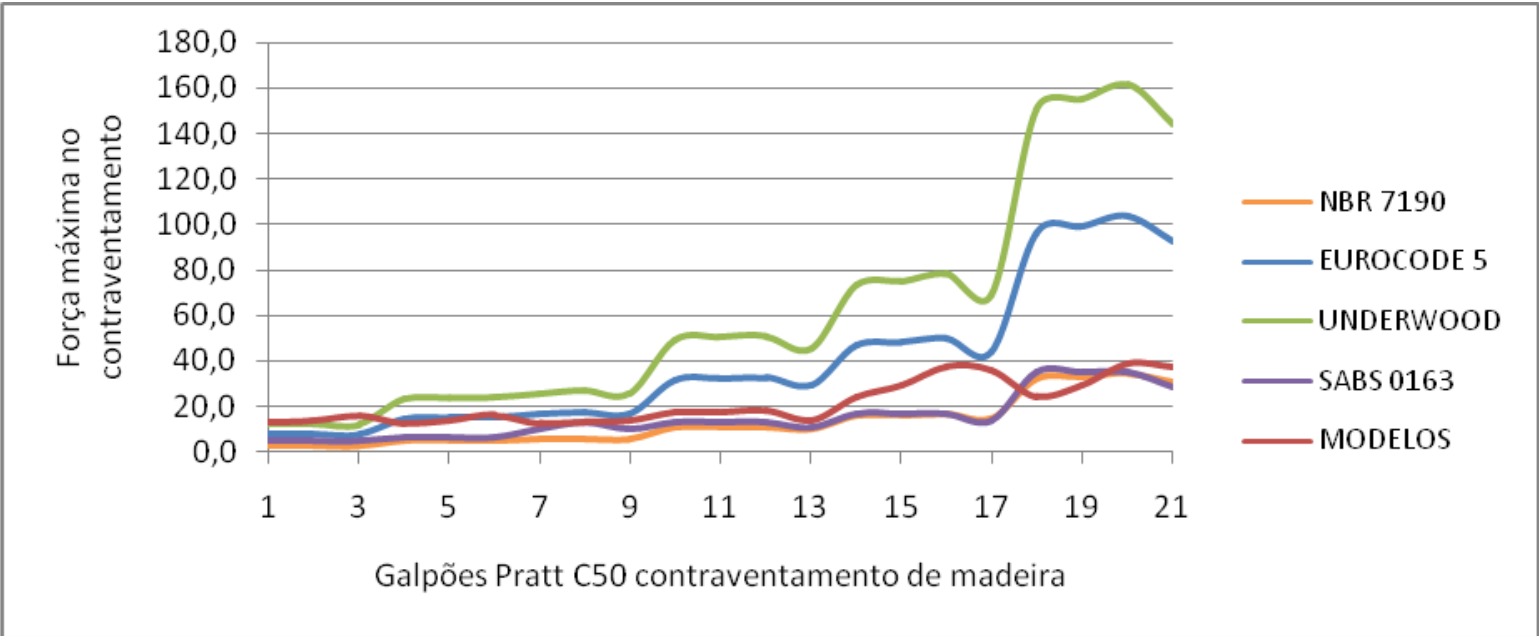

\section{Gráfico 17 - Colunas comparativas do método NBR 7190 (PRATT C50 madeira)}

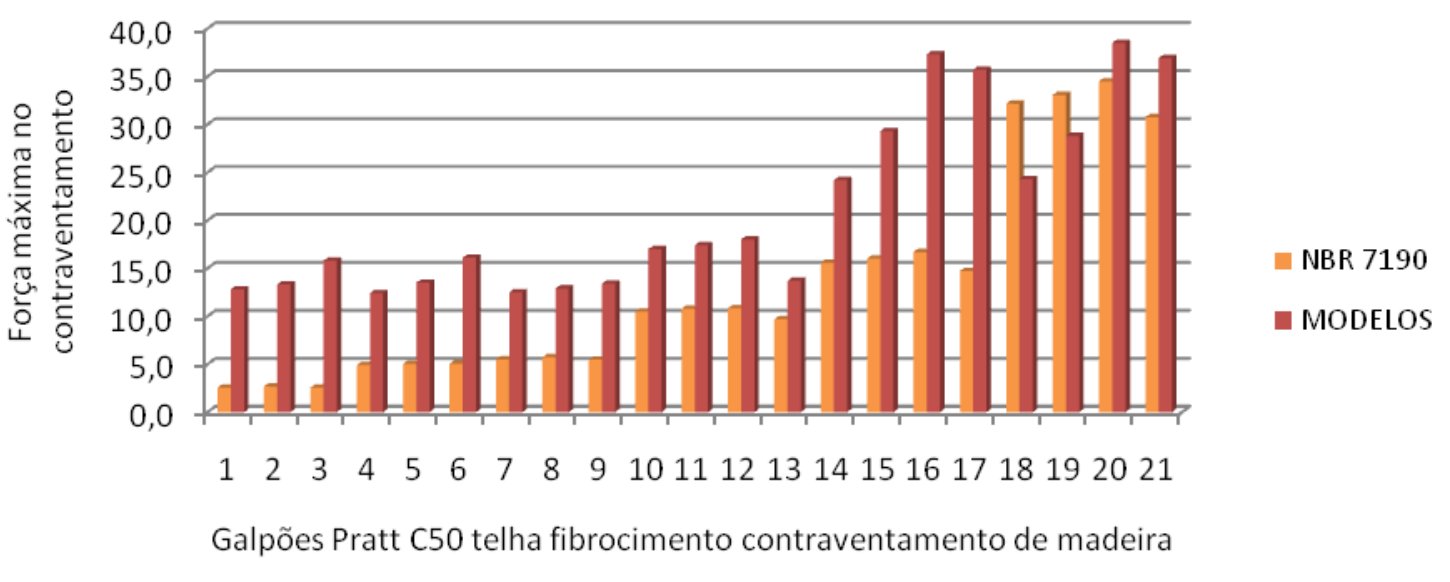

Gráfico 18 - Colunas comparativas do método EUROCODE 5 (PRATT C50 madeira)

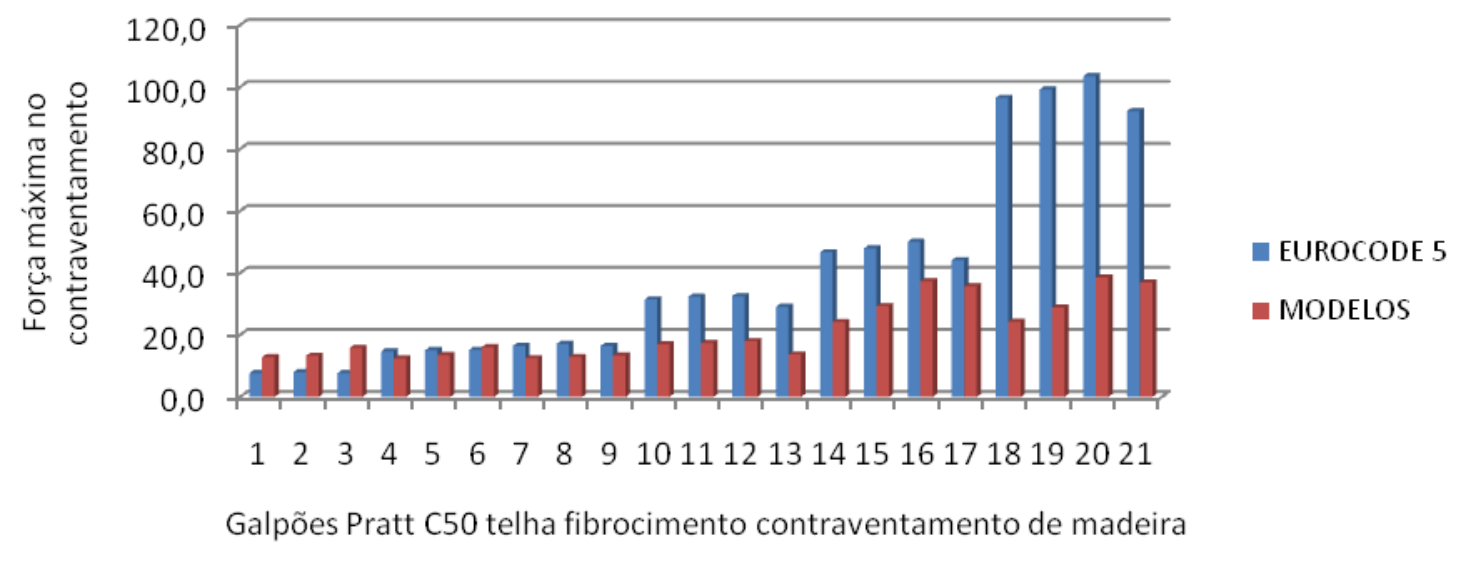


Gráfico 19 - Colunas comparativas do método UNDERWOOD (PRATT C50 madeira)

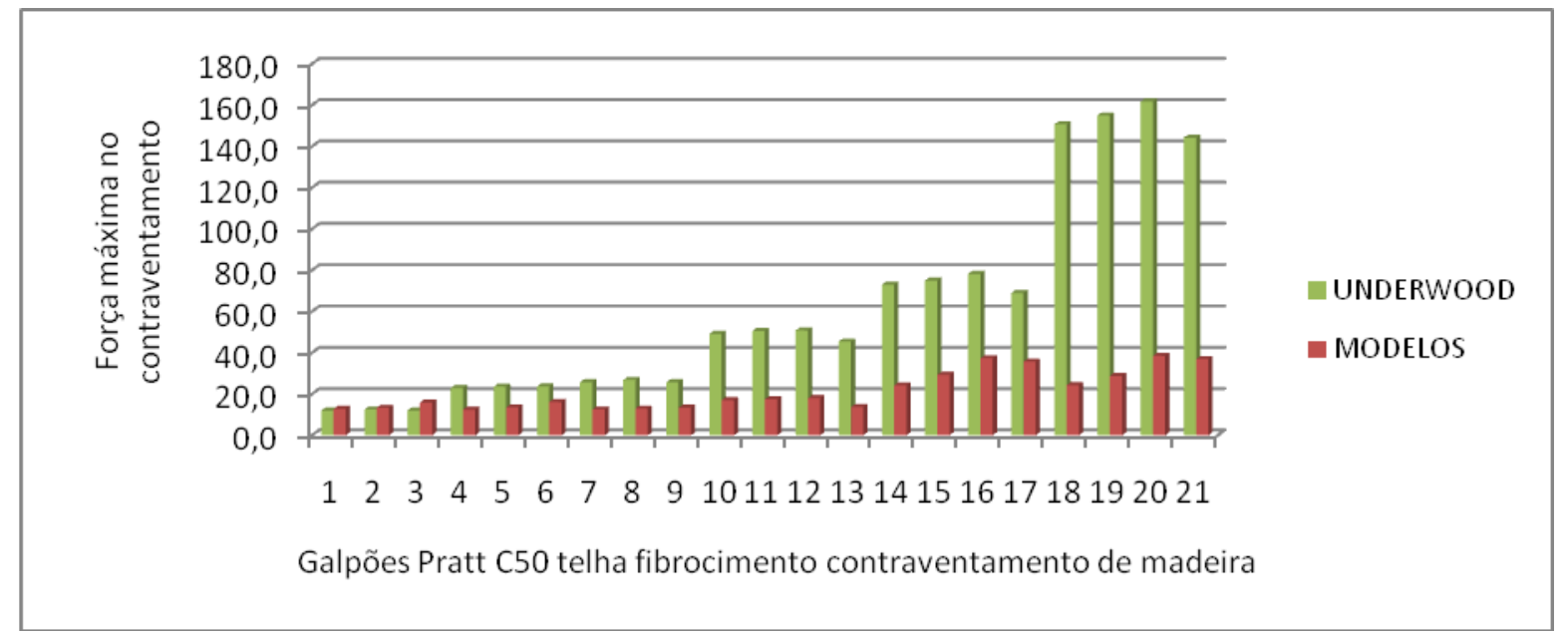

Gráfico 20 - Colunas comparativas do método SABS 0163 (PRATT C50 madeira)

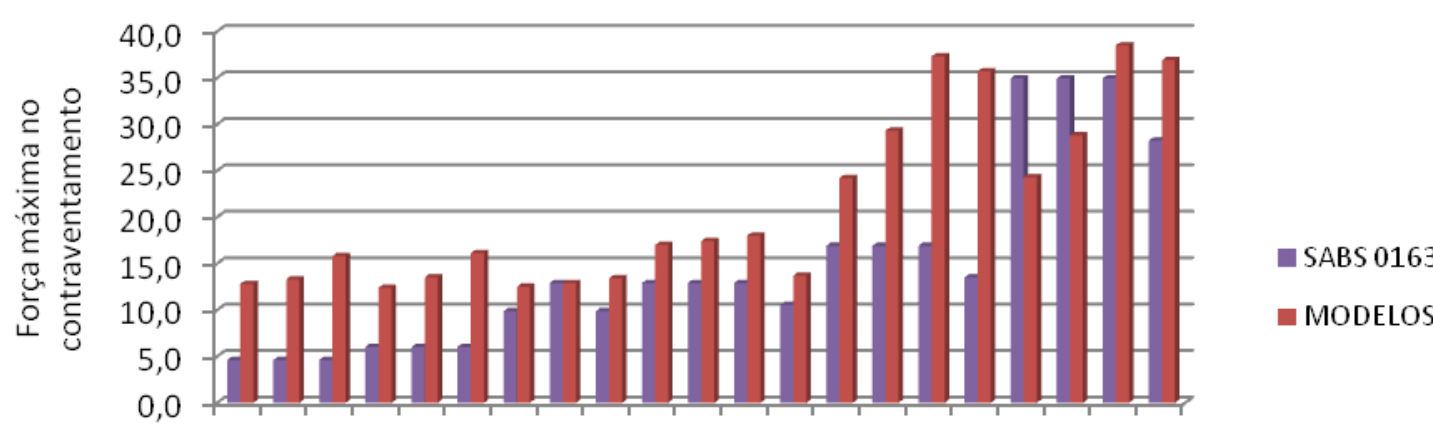

$\begin{array}{lllllllllllll}1 & 2 & 3 & 4 & 5 & 6 & 7 & 8 & 9 & 1011 & 12131415161718192021\end{array}$

Galpões Pratt C50 telha fibrocimento contraventamento de madeira 


\subsection{Treliças PRATT C30}

Tabela 5.17 - Comparativo entre o método da NBR 7190 (1997) e os modelos tridimensionais (PRATT C30)

\begin{tabular}{|c|c|c|c|c|c|c|c|c|c|c|c|c|}
\hline \multicolumn{13}{|c|}{ GEOMETRIAS DAS TRELIÇAS } \\
\hline \multicolumn{2}{|c|}{ L (vão) } & 12 & 12 & 12 & 18 & 18 & 18 & 18 & 24 & 24 & 24 & 24 \\
\hline \multicolumn{2}{|c|}{ h (altura central) } & 1,5 & 1,5 & 1,5 & 2,25 & 2,25 & 2,25 & 2,25 & 3 & 3 & 3 & 3 \\
\hline \multicolumn{2}{|c|}{ z (altura do pilar) } & 3 & 4 & 6 & 3 & 4 & 6 & 6 & 3 & 4 & 6 & 6 \\
\hline \multicolumn{2}{|c|}{ e (dist. entre treliças) } & 3 & 3 & 3 & 3 & 3 & 3 & 4 & 3 & 3 & 3 & 4 \\
\hline \multicolumn{13}{|c|}{ MÉTODO NBR 7190 (1997) } \\
\hline \multicolumn{2}{|c|}{ Compressão $_{\text {máx }}(\mathrm{kN})$} & 83,0 & 86,4 & 90,9 & 155,9 & 161,1 & 168,3 & 205,2 & 238,8 & 244,5 & 254,7 & 305,2 \\
\hline \multirow{6}{*}{$F_{d}(\mathbf{k N})$} & $\mathrm{F}_{1 \mathrm{~d}}$ & 0,6 & 0,6 & 0,6 & 1,0 & 1,1 & 1,1 & 1,4 & 1,6 & 1,6 & 1,7 & 2,0 \\
\hline & $\mathrm{n}=7$ & 2,6 & 2,7 & 2,8 & 4,9 & 5,0 & 5,2 & - & - & - & - & - \\
\hline & $\mathrm{n}=11$ & - & - & - & - & - & - & 10,0 & - & - & - & 14,9 \\
\hline & $\mathrm{n}=15$ & 5,5 & 5,8 & 6,1 & 10,4 & 10,7 & 11,2 & - & 15,9 & 16,3 & 17,0 & \\
\hline & $n=23$ & - & - & - & - & - & - & - & - & - & - & 31,2 \\
\hline & $\mathrm{n}=31$ & - & - & - & - & - & - & - & 32,9 & 33,7 & 35,1 & - \\
\hline \multicolumn{13}{|c|}{ MODELOS NUMÉRICOS } \\
\hline \multicolumn{2}{|c|}{ Compressão $_{\text {máx }}(\mathrm{kN})$} & 89,9 & 93,5 & 98,1 & 165,1 & 170,9 & 180,5 & 224,4 & 248,9 & 256,9 & 266,2 & 324,9 \\
\hline \multirow{5}{*}{$\begin{array}{c}\mathbf{N}_{\mathrm{d}, \mathrm{t}}(\mathbf{k N}) \\
\text { Terças }\end{array}$} & $\mathrm{n}=7$ & 12,4 & 16,3 & 23,5 & 8,4 & 11,0 & 16,1 & - & - & - & - & - \\
\hline & $\mathrm{n}=11$ & - & - & - & - & - & - & 19,7 & - & - & - & 59,4 \\
\hline & $\mathrm{n}=15$ & 12,8 & 16,9 & 23,8 & 9,4 & 12,1 & 18,4 & - & 33,4 & 43,6 & 60,0 & - \\
\hline & $\mathrm{n}=23$ & - & - & - & - & - & - & - & - & - & - & 59,2 \\
\hline & $\mathrm{n}=31$ & - & - & - & - & - & & - & 32,2 & 41,1 & 59,7 & \\
\hline \multicolumn{13}{|c|}{ COMPARATIVO Galpão/Treliça } \\
\hline \multicolumn{2}{|c|}{ Compressãomáx $_{\text {maN})}$} & 1,08 & 1,08 & 1,08 & 1,06 & 1,06 & $\mathbf{1 , 0 7}$ & 1,09 & 1,04 & 1,05 & 1,05 & 1,06 \\
\hline \multirow{5}{*}{$\mathbf{N}_{\mathrm{d}, \mathrm{t}} / \mathbf{F}_{\mathbf{d}}$} & $\mathrm{n}=7$ & 4,80 & 6,06 & 8,31 & 1,73 & 2,19 & 3,07 & - & - & - & - & - \\
\hline & $\mathrm{n}=11$ & - & - & - & - & - & - & 1,96 & - & - & - & 3,98 \\
\hline & $\mathrm{n}=15$ & 2,31 & 2,93 & 3,93 & 0,90 & 1,13 & 1,64 & & 2,10 & 2,67 & 3,53 & \\
\hline & $\mathrm{n}=23$ & - & - & - & - & - & - & - & - & - & - & 1,90 \\
\hline & $\mathrm{n}=31$ & - & - & - & - & - & - & - & 0,98 & 1,22 & 1,70 & - \\
\hline
\end{tabular}


Tabela 5.18 - Comparativo entre o método do EUROCODE 5 e os modelos tridimensionais (PRATT C30)

\begin{tabular}{|c|c|c|c|c|c|c|c|c|c|c|c|c|}
\hline \multicolumn{13}{|c|}{ GEOMETRIAS DAS TRELIÇAS } \\
\hline \multicolumn{2}{|c|}{ L (vão) } & 12 & 12 & 12 & 18 & 18 & 18 & 18 & 24 & 24 & 24 & 24 \\
\hline \multicolumn{2}{|c|}{ h (altura central) } & 1,5 & 1,5 & 1,5 & 2,25 & 2,25 & 2,25 & 2,25 & 3 & 3 & 3 & 3 \\
\hline \multicolumn{2}{|c|}{ z (altura do pilar) } & 3 & 4 & 6 & 3 & 4 & 6 & 6 & 3 & 4 & 6 & 6 \\
\hline \multicolumn{2}{|c|}{ e (dist. entre treliças) } & 3 & 3 & 3 & 3 & 3 & 3 & 4 & 3 & 3 & 3 & 4 \\
\hline \multicolumn{13}{|c|}{ MÉTODO EUROCODE 5} \\
\hline \multicolumn{2}{|c|}{ Compressão máx $_{\text {mN) }}$} & 83,0 & 86,4 & 90,9 & 155,9 & 161,1 & 168,3 & 205,2 & 238,8 & 244,5 & 254,7 & 305,2 \\
\hline \multirow{6}{*}{$F_{d}(k N)$} & $\mathrm{F}_{1 \mathrm{~d}}$ & 1,7 & 1,7 & 1,8 & 3,1 & 3,2 & 3,4 & 4,1 & 4,8 & 4,9 & 5,1 & 6,1 \\
\hline & $\mathrm{n}=7$ & 7,7 & 8,1 & 8,5 & 14,6 & 15,0 & 15,7 & - & - & - & - & - \\
\hline & $\mathrm{n}=11$ & - & - & - & - & - & - & 30,1 & - & - & - & 44,8 \\
\hline & $\mathrm{n}=15$ & 16,6 & 17,3 & 18,2 & 31,2 & 32,2 & 33,7 & & 47,8 & 48,9 & 50,9 & \\
\hline & $n=23$ & - & - & - & - & - & - & - & - & - & - & 93,6 \\
\hline & $\mathrm{n}=31$ & - & - & - & - & - & - & - & 98,7 & 101,1 & 105,3 & - \\
\hline \multicolumn{13}{|c|}{ MODELOS NUMÉRICOS } \\
\hline \multicolumn{2}{|c|}{ Compressãomáx $(\mathrm{kN})$} & 89,9 & 93,5 & 98,1 & 165,1 & 170,9 & 180,5 & 224,4 & 248,9 & 256,9 & 266,2 & 324,9 \\
\hline \multirow{5}{*}{$\begin{array}{c}\mathbf{N}_{\mathrm{d}, \mathrm{t}}(\mathbf{k N}) \\
\text { Terças }\end{array}$} & $\mathrm{n}=7$ & 12,4 & 16,3 & 23,5 & 8,4 & 11,0 & 16,1 & - & - & - & - & - \\
\hline & $\mathrm{n}=11$ & - & - & - & - & - & - & 19,7 & - & - & - & 59,4 \\
\hline & $\mathrm{n}=15$ & 12,8 & 16,9 & 23,8 & 9,4 & 12,1 & 18,4 & & 33,4 & 43,6 & 60,0 & \\
\hline & $n=23$ & - & - & - & - & - & - & - & - & - & - & 59,2 \\
\hline & $\mathrm{n}=31$ & - & - & - & - & - & - & - & 32,2 & 41,1 & 59,7 & - \\
\hline \multicolumn{13}{|c|}{ COMPARATIVO Galpão/Treliça } \\
\hline \multicolumn{2}{|c|}{ Compressão máx $_{\text {mN) }}$} & 1,08 & 1,08 & 1,08 & 1,06 & 1,06 & $\mathbf{1 , 0 7}$ & 1,09 & 1,04 & 1,05 & 1,05 & 1,06 \\
\hline \multirow{5}{*}{$\mathbf{N}_{\mathrm{d}, \mathrm{t}} / \mathbf{F}_{\mathbf{d}}$} & $\mathrm{n}=7$ & 1,60 & 2,02 & 2,77 & 0,58 & 0,73 & 1,02 & & - & - & - & \\
\hline & $\mathrm{n}=11$ & - & - & - & - & - & - & 0,65 & - & - & - & 1,33 \\
\hline & $\mathrm{n}=15$ & 0,77 & 0,98 & 1,31 & $\mathbf{0 , 3 0}$ & 0,38 & 0,55 & & $\mathbf{0 , 7 0}$ & 0,89 & 1,18 & \\
\hline & $n=23$ & - & - & - & - & - & - & - & - & - & - & 0,63 \\
\hline & $\mathrm{n}=31$ & - & - & - & - & - & - & - & 0,33 & 0,41 & 0,57 & - \\
\hline
\end{tabular}


Tabela 5.19 - Comparativo entre o método de UNDERWOOD (2000) e os modelos tridimensionais (PRATT C30)

\begin{tabular}{|c|c|c|c|c|c|c|c|c|c|c|c|c|}
\hline \multicolumn{13}{|c|}{ GEOMETRIAS DAS TRELIÇAS } \\
\hline \multicolumn{2}{|c|}{ L (vão) } & 12 & 12 & 12 & 18 & 18 & 18 & 18 & 24 & 24 & 24 & 24 \\
\hline \multicolumn{2}{|c|}{ h (altura central) } & 1,5 & 1,5 & 1,5 & 2,25 & 2,25 & 2,25 & 2,25 & 3 & 3 & 3 & 3 \\
\hline \multicolumn{2}{|c|}{ z (altura do pilar) } & 3 & 4 & 6 & 3 & 4 & 6 & 6 & 3 & 4 & 6 & 6 \\
\hline \multicolumn{2}{|c|}{ e (dist. entre treliças) } & 3 & 3 & 3 & 3 & 3 & 3 & 4 & 3 & 3 & 3 & 4 \\
\hline \multicolumn{13}{|c|}{ MÉTODO UNDERWOOD (2000) } \\
\hline \multicolumn{2}{|c|}{ Compressãomáx $(\mathrm{kN})$} & 83,0 & 86,4 & 90,9 & 155,9 & 161,1 & 168,3 & 205,2 & 238,8 & 244,5 & 254,7 & 305,2 \\
\hline \multirow{6}{*}{$F_{d}(k N)$} & $\mathrm{F}_{1 \mathrm{~d}}$ & 2,6 & 2,7 & 2,8 & 4,9 & 5,0 & 5,3 & 6,4 & 7,5 & 7,6 & 8,0 & 9,5 \\
\hline & $\mathrm{n}=7$ & 12,1 & 12,6 & 13,3 & 22,7 & 23,5 & 24,5 & - & - & - & - & - \\
\hline & $\mathrm{n}=11$ & - & - & - & - & - & - & 47,0 & - & - & - & 69,9 \\
\hline & $\mathrm{n}=15$ & 25,9 & 27,0 & 28,4 & 48,7 & 50,3 & 52,6 & - & 74,6 & 76,4 & 79,6 & \\
\hline & $n=23$ & - & - & - & - & - & - & - & - & - & - & 146,2 \\
\hline & $\mathrm{n}=31$ & - & - & - & - & - & - & - & 154,2 & 157,9 & 164,5 & - \\
\hline \multicolumn{13}{|c|}{ MODELOS NUMÉRICOS } \\
\hline \multicolumn{2}{|c|}{ Compressão máx $_{\text {mN) }}$} & 89,9 & 93,5 & 98,1 & 165,1 & 170,9 & 180,5 & 224,4 & 248,9 & 256,9 & 266,2 & 324,9 \\
\hline \multirow{5}{*}{$\begin{array}{c}\mathbf{N}_{\mathbf{d}, \mathrm{t}}(\mathbf{k N}) \\
\text { Terças }\end{array}$} & $\mathrm{n}=7$ & 12,4 & 16,3 & 23,5 & 8,4 & 11,0 & 16,1 & - & - & - & & \\
\hline & $\mathrm{n}=11$ & - & - & - & - & - & - & 19,7 & - & - & - & 59,4 \\
\hline & $\mathrm{n}=15$ & 12,8 & 16,9 & 23,8 & 9,4 & 12,1 & 18,4 & - & 33,4 & 43,6 & 60,0 & - \\
\hline & $n=23$ & - & - & - & - & - & - & - & - & - & - & 59,2 \\
\hline & $\mathrm{n}=31$ & - & - & - & - & - & - & - & 32,2 & 41,1 & 59,7 & - \\
\hline \multicolumn{13}{|c|}{ COMPARATIVO Galpão/Treliça } \\
\hline \multicolumn{2}{|c|}{ Compressãomáx $_{\text {maN) }}$} & 1,08 & 1,08 & 1,08 & 1,06 & 1,06 & $\mathbf{1 , 0 7}$ & 1,09 & 1,04 & 1,05 & 1,05 & 1,06 \\
\hline \multirow{5}{*}{$\mathbf{N}_{\mathrm{d}, \mathrm{t}} / \mathbf{F}_{\mathbf{d}}$} & $\mathrm{n}=7$ & 1,02 & 1,29 & 1,77 & 0,37 & 0,47 & 0,66 & - & - & - & - & - \\
\hline & $\mathrm{n}=11$ & - & - & - & - & - & - & 0,42 & - & - & - & 0,85 \\
\hline & $\mathrm{n}=15$ & 0,49 & 0,63 & 0,84 & $\mathbf{0 , 1 9}$ & 0,24 & 0,35 & - & 0,45 & 0,57 & 0,75 & - \\
\hline & $n=23$ & - & - & - & - & - & - & - & - & - & - & 0,40 \\
\hline & $\mathrm{n}=31$ & - & - & - & - & - & - & - & 0,21 & 0,26 & 0,36 & - \\
\hline
\end{tabular}


Tabela 5.20 - Comparativo entre o método do SABS 0163 e os modelos tridimensionais (PRATT C30)

\begin{tabular}{|c|c|c|c|c|c|c|c|c|c|c|c|c|}
\hline \multicolumn{13}{|c|}{ GEOMETRIAS DAS TRELIÇAS } \\
\hline \multicolumn{2}{|c|}{ L (vão) } & 12 & 12 & 12 & 18 & 18 & 18 & 18 & 24 & 24 & 24 & 24 \\
\hline \multicolumn{2}{|c|}{ h (altura central) } & 1,5 & 1,5 & 1,5 & 2,25 & 2,25 & 2,25 & 2,25 & 3 & 3 & 3 & 3 \\
\hline \multicolumn{2}{|c|}{ z (altura do pilar) } & 3 & 4 & 6 & 3 & 4 & 6 & 6 & 3 & 4 & 6 & 6 \\
\hline \multicolumn{2}{|c|}{ e (dist. entre treliças) } & 3 & 3 & 3 & 3 & 3 & 3 & 4 & 3 & 3 & 3 & 4 \\
\hline \multicolumn{13}{|c|}{ MÉTODO SABS 0163} \\
\hline \multicolumn{2}{|c|}{ Compressão $_{\text {perm }}(\mathrm{kN})$} & \multicolumn{3}{|c|}{38,2} & \multicolumn{3}{|c|}{84,4} & 93,2 & \multicolumn{3}{|c|}{139,2} & 151,6 \\
\hline \multirow{6}{*}{$F_{d}(\mathbf{k N})$} & $\mathrm{F}_{1 \mathrm{~d}}$ & \multicolumn{3}{|c|}{1,0} & \multicolumn{3}{|c|}{1,4} & 1,6 & \multicolumn{3}{|c|}{1,7} & 1,9 \\
\hline & $\mathrm{n}=7$ & 4,5 & 4,5 & 4,5 & 6,6 & 6,6 & 6,6 & - & - & - & - & - \\
\hline & $\mathrm{n}=11$ & - & - & - & - & - & - & 11,4 & - & - & - & 13,9 \\
\hline & $\mathrm{n}=15$ & 9,6 & 14,1 & 9,6 & 14,1 & 14,1 & 14,1 & & 17,4 & 17,4 & 17,4 & \\
\hline & $\mathrm{n}=23$ & - & - & - & - & - & - & - & - & - & - & 29,1 \\
\hline & $\mathrm{n}=31$ & - & - & - & - & - & - & - & 36,0 & 36,0 & 36,0 & - \\
\hline \multicolumn{13}{|c|}{ MODELOS NUMÉRICOS } \\
\hline \multicolumn{2}{|c|}{ Compressão $_{\text {máx }}(\mathrm{kN})$} & 89,9 & 93,5 & 98,1 & 165,1 & 170,9 & 180,5 & 224,4 & 248,9 & 256,9 & 266,2 & 324,9 \\
\hline \multirow{5}{*}{$\begin{array}{c}\mathbf{N}_{\mathrm{d}, \mathrm{t}}(\mathbf{k N}) \\
\text { Terças }\end{array}$} & $\mathrm{n}=7$ & 12,4 & 16,3 & 23,5 & 8,4 & 11,0 & 16,1 & - & - & - & - & - \\
\hline & $\mathrm{n}=11$ & - & - & - & - & - & - & 19,7 & - & - & - & 59,4 \\
\hline & $\mathrm{n}=15$ & 12,8 & 16,9 & 23,8 & 9,4 & 12,1 & 18,4 & & 33,4 & 43,6 & 60,0 & \\
\hline & $\mathrm{n}=23$ & - & - & - & - & - & - & - & - & - & - & 59,2 \\
\hline & $\mathrm{n}=31$ & - & - & & - & - & - & & 32,2 & 41,1 & 59,7 & \\
\hline \multicolumn{13}{|c|}{ COMPARATIVO Galpão/Treliça } \\
\hline \multirow{5}{*}{$\mathbf{N}_{\mathrm{d}, \mathrm{t}} / \mathbf{F}_{\mathbf{d}}$} & $\mathrm{n}=7$ & 2,78 & 3,66 & 5,27 & 1,28 & 1,68 & 2,45 & & - & - & & \\
\hline & $\mathrm{n}=11$ & - & - & - & - & - & - & 1,73 & - & - & - & 4,27 \\
\hline & $\mathrm{n}=15$ & 1,34 & 1,20 & 2,49 & 0,67 & $\mathbf{0 , 8 6}$ & 1,31 & - & 1,92 & 2,51 & 3,45 & - \\
\hline & $\mathrm{n}=23$ & - & - & - & - & - & - & - & - & - & - & 2,04 \\
\hline & $\mathrm{n}=31$ & - & - & - & - & - & - & - & $\mathbf{0 , 9 0}$ & 1,14 & 1,66 & - \\
\hline
\end{tabular}


Gráfico 21 - Linhas comparativas dos métodos (PRATT C30)

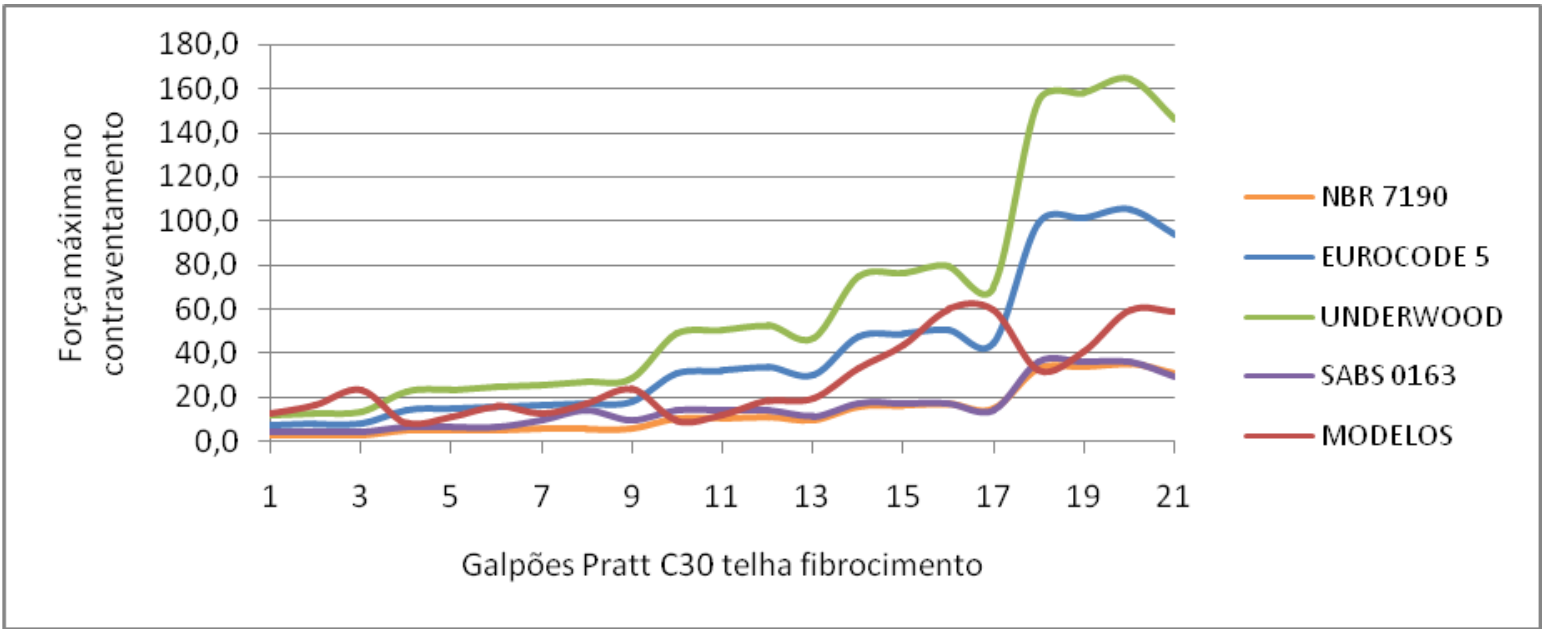

Gráfico 22 - Colunas comparativas do método NBR 7190 (PRATT C30)

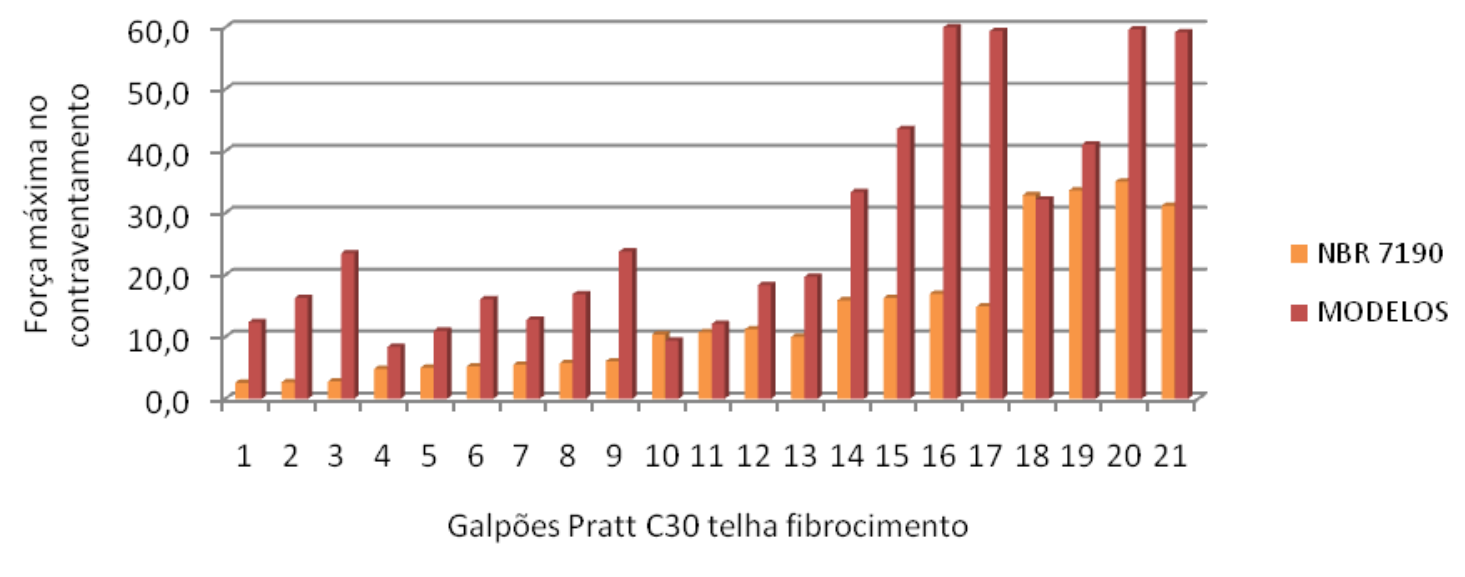

Gráfico 23 - Colunas comparativas do método EUROCODE 5 (PRATT C30)

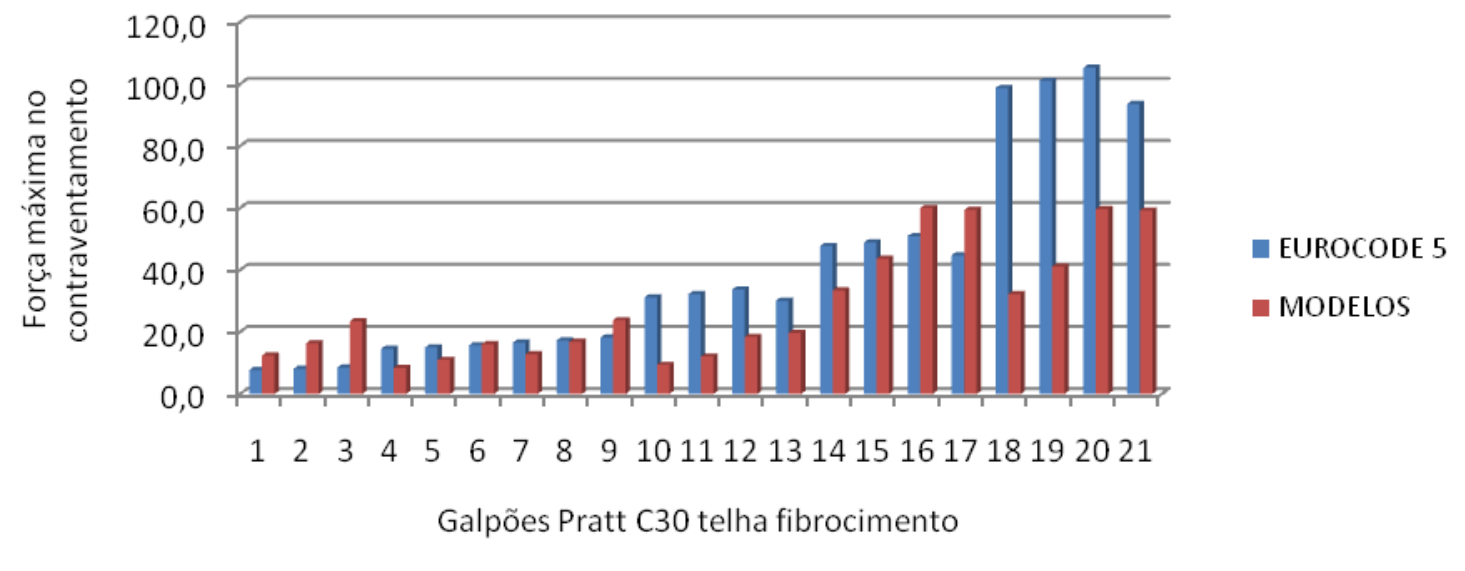


Gráfico 24 - Colunas comparativas do método UNDERWOOD (PRATT C30)

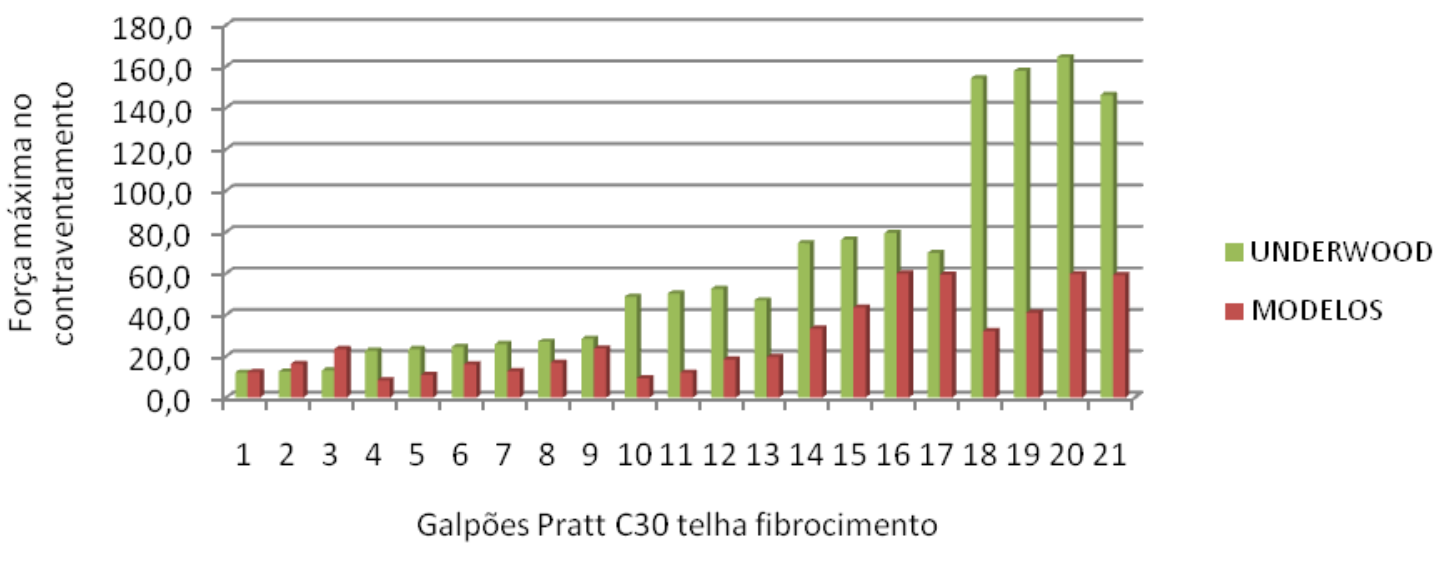

Gráfico 25 - Colunas comparativas do método SABS 0163 (PRATT C30)

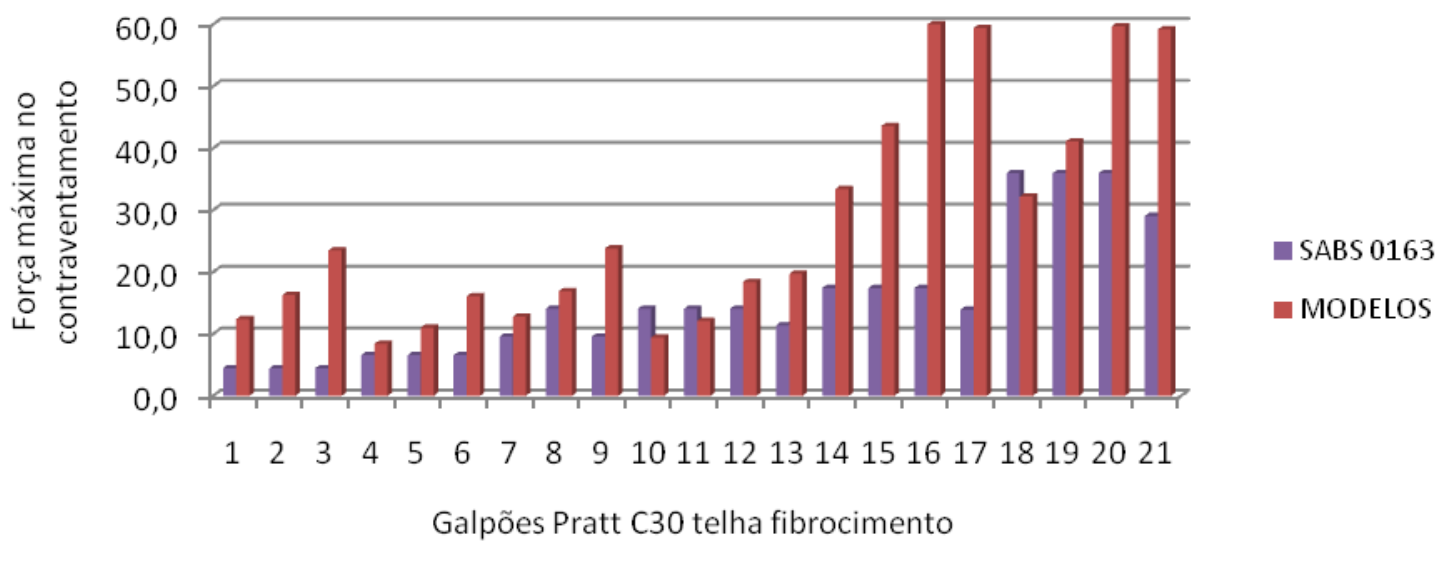




\subsection{Treliças PRATT C50 telha metálica}

Tabela 5.21 - Comparativo entre o método da NBR 7190 (1997) e os modelos tridimensionais (PRATT C50 telha metálica)

\begin{tabular}{|c|c|c|c|c|c|c|c|c|c|c|c|c|}
\hline & & & GE & OME] & ГRIAS & DAS 1 & RELI & ÇAS & & & & \\
\hline L (v & & 12 & 12 & 12 & 18 & 18 & 18 & 18 & 24 & 24 & 24 & 24 \\
\hline h (altura & entral) & 1,5 & 1,5 & 1,5 & 2,25 & 2,25 & 2,25 & 2,25 & 3 & 3 & 3 & 3 \\
\hline z (altura & pilar) & 3 & 4 & 6 & 3 & 4 & 6 & 6 & 3 & 4 & 6 & 6 \\
\hline e (dist. entr & treliças) & 3 & 3 & 3 & 3 & 3 & 3 & 4 & 3 & 3 & 3 & 4 \\
\hline & & & & MÉTO & DO NI & BR 719 & $0(199$ & & & & & \\
\hline Compressã & máx $(\mathrm{kN})$ & 65,9 & 68,3 & 74,0 & 116,8 & 120,7 & 126,5 & 160,3 & 185,3 & 190,8 & 202,5 & 244,6 \\
\hline & $\mathrm{F}_{1 \mathrm{~d}}$ & 0,4 & 0,5 & 0,5 & 0,8 & 0,8 & 0,8 & 1,1 & 1,2 & 1,3 & 1,4 & 1,6 \\
\hline & $\begin{array}{ll}\mathrm{n} & 7\end{array}$ & 2,1 & 2,1 & 2,3 & 3,6 & 3,8 & 3,9 & - & - & - & - & - \\
\hline$F_{d}(k N)$ & n 11 & - & - & - & - & - & - & 7,8 & - & - & - & 12,0 \\
\hline $1 d(n)$ & n 15 & 4,4 & 4,6 & 4,9 & 7,8 & 8,0 & 8,4 & - & 12,4 & 12,7 & 13,5 & - \\
\hline & n 23 & - & - & - & - & - & - & - & - & - & - & 25,0 \\
\hline & n 31 & - & - & - & - & - & - & - & 25,5 & 26,3 & 27,9 & - \\
\hline & & & & MODI & ELOS & NUMÉ & RICO & & & & & \\
\hline Compressã & máx $(\mathrm{kN})$ & 72,2 & 75,9 & 105,7 & 121,0 & 134,1 & 134,1 & 171,4 & 195,0 & 202,9 & 212,7 & 258,4 \\
\hline & $\mathrm{n}=7$ & 12,4 & 16,3 & 23,5 & 13,0 & 15,7 & 18,4 & - & - & & - & - \\
\hline & $\mathrm{n}=11$ & - & - & - & - & - & - & 19,1 & - & - & - & 59,3 \\
\hline $\mathbf{N}_{\mathrm{d}, \mathrm{t}}(\mathrm{kN})$ & $\mathrm{n}=15$ & 12,7 & 16,8 & 24,0 & 16,2 & 18,0 & 20,8 & - & 33,2 & 43,1 & 60,0 & - \\
\hline & $\mathrm{n}=23$ & - & - & - & - & - & - & - & - & - & - & 59,2 \\
\hline & $\mathrm{n}=31$ & - & - & - & - & - & - & - & 32,0 & 40,9 & 59,6 & - \\
\hline & & & $\mathrm{CO}$ & MPA & RATIV & O Gal & pão/Tru & liça & & & & \\
\hline Compressã & máx $(\mathrm{kN})$ & 1,10 & 1,11 & 1,43 & 1,04 & 1,11 & 1,06 & $\mathbf{1 , 0 7}$ & 1,05 & 1,06 & 1,05 & 1,06 \\
\hline & $\mathrm{n}=7$ & 6,05 & 7,67 & 10,21 & 3,58 & 4,18 & 4,68 & & - & & - & - \\
\hline & $\mathrm{n}=11$ & - & - & - & - & - & - & 2,44 & - & - & - & 4,96 \\
\hline $\mathbf{N}_{\mathrm{d}, \mathrm{t}} / \mathbf{F}_{\mathbf{d}}$ & $\mathrm{n}=15$ & 2,89 & 3,69 & 4,86 & 2,08 & 2,24 & 2,47 & - & 2,69 & 3,39 & 4,44 & - \\
\hline & $\mathrm{n}=23$ & - & - & - & - & - & - & - & - & - & - & 2,37 \\
\hline & $\mathrm{n}=31$ & - & - & - & - & - & - & - & 1,25 & 1,56 & 2,14 & - \\
\hline
\end{tabular}


Tabela 5.22 - Comparativo entre o método do EUROCODE 5 e os modelos tridimensionais (PRATT C50 telha metálica)

\begin{tabular}{|c|c|c|c|c|c|c|c|c|c|c|c|c|}
\hline \multicolumn{13}{|c|}{ GEOMETRIAS DAS TRELIÇAS } \\
\hline \multicolumn{2}{|c|}{ L (vão) } & 12 & 12 & 12 & 18 & 18 & 18 & 18 & 24 & 24 & 24 & 24 \\
\hline \multicolumn{2}{|c|}{ h (altura central) } & 1,5 & 1,5 & 1,5 & 2,25 & 2,25 & 2,25 & 2,25 & 3 & 3 & 3 & 3 \\
\hline \multicolumn{2}{|c|}{ z (altura do pilar) } & 3 & 4 & 6 & 3 & 4 & 6 & 6 & 3 & 4 & 6 & 6 \\
\hline \multicolumn{2}{|c|}{ e (dist. entre treliças) } & 3 & 3 & 3 & 3 & 3 & 3 & 4 & 3 & 3 & 3 & 4 \\
\hline \multicolumn{13}{|c|}{ MÉTODO EUROCODE 5} \\
\hline \multicolumn{2}{|c|}{ Compressão $_{\text {máx }}(\mathrm{kN})$} & 65,9 & 68,3 & 74,0 & 116,8 & 120,7 & 126,5 & 160,3 & 185,3 & 190,8 & 202,5 & 244,6 \\
\hline \multirow{6}{*}{$F_{d}(k N)$} & $\mathrm{F}_{1 \mathrm{~d}}$ & 1,3 & 1,4 & 1,5 & 2,3 & 2,4 & 2,5 & 3,2 & 3,7 & 3,8 & 4,1 & 4,9 \\
\hline & $\begin{array}{ll}\mathrm{n} & 7\end{array}$ & 6,2 & 6,4 & 6,9 & 10,9 & 11,3 & 11,8 & - & - & - & - & - \\
\hline & n 11 & - & - & - & - & - & - & 23,5 & - & - & - & 35,9 \\
\hline & n 15 & 13,2 & 13,7 & 14,8 & 23,4 & 24,1 & 25,3 & - & 37,1 & 38,2 & 40,5 & - \\
\hline & n 23 & - & - & - & - & - & - & - & - & - & - & 75,0 \\
\hline & n 31 & - & - & - & - & - & - & - & 76,6 & 78,9 & 83,7 & - \\
\hline \multicolumn{13}{|c|}{ MODELOS NUMÉRICOS } \\
\hline \multicolumn{2}{|c|}{ Compressão $_{\text {máx }}(\mathrm{kN})$} & 72,2 & 75,9 & 105,7 & 121,0 & 134,1 & 134,1 & 171,4 & 195,0 & 202,9 & 212,7 & 258,4 \\
\hline \multirow{5}{*}{$\begin{array}{c}\mathbf{N}_{\mathrm{d}, \mathrm{t}}(\mathbf{k N}) \\
\text { Terças }\end{array}$} & $\mathrm{n}=7$ & 12,4 & 16,3 & 23,5 & 13,0 & 15,7 & 18,4 & - & - & - & - & - \\
\hline & $\mathrm{n}=11$ & - & - & - & - & - & - & 19,1 & - & - & - & 59,3 \\
\hline & $\mathrm{n}=15$ & 12,7 & 16,8 & 24,0 & 16,2 & 18,0 & 20,8 & - & 33,2 & 43,1 & 60,0 & - \\
\hline & $\mathrm{n}=23$ & - & - & - & - & - & - & - & - & - & - & 59,2 \\
\hline & $\mathrm{n}=31$ & - & - & - & - & - & - & - & 32,0 & 40,9 & 59,6 & - \\
\hline \multicolumn{13}{|c|}{ COMPARATIVO Galpão/Treliça } \\
\hline \multicolumn{2}{|c|}{ Compressão máx $_{(\mathrm{kN})}$} & 1,10 & 1,11 & 1,43 & 1,04 & 1,11 & 1,06 & 1,07 & 1,05 & 1,06 & 1,05 & 1,06 \\
\hline \multirow{5}{*}{$\mathbf{N}_{\mathrm{d}, \mathrm{t}} / \mathbf{F}_{\mathbf{d}}$} & $\mathrm{n}=7$ & 2,02 & 2,56 & 3,40 & 1,19 & 1,39 & 1,56 & - & - & - & & - \\
\hline & $\mathrm{n}=11$ & - & - & - & - & - & - & $\mathbf{0 , 8 1}$ & - & - & - & 1,65 \\
\hline & $\mathrm{n}=15$ & 0,96 & 1,23 & 1,62 & 0,69 & 0,75 & 0,82 & - & 0,90 & 1,13 & 1,48 & \\
\hline & $n=23$ & - & - & - & - & - & - & - & - & - & - & 0,79 \\
\hline & $\mathrm{n}=31$ & - & - & - & & - & - & - & 0,42 & 0,52 & 0,71 & - \\
\hline
\end{tabular}


Tabela 5.23 - Comparativo entre o método de UNDERWOOD (2000) e os modelos tridimensionais (PRATT C50 telha metálica)

\begin{tabular}{|c|c|c|c|c|c|c|c|c|c|c|c|c|}
\hline \multicolumn{13}{|c|}{ GEOMETRIAS DAS TRELIÇAS } \\
\hline \multicolumn{2}{|c|}{ L (vão) } & 12 & 12 & 12 & 18 & 18 & 18 & 18 & 24 & 24 & 24 & 24 \\
\hline \multicolumn{2}{|c|}{ h (altura central) } & 1,5 & 1,5 & 1,5 & 2,25 & 2,25 & 2,25 & 2,25 & 3 & 3 & 3 & 3 \\
\hline \multicolumn{2}{|c|}{ z (altura do pilar) } & 3 & 4 & 6 & 3 & 4 & 6 & 6 & 3 & 4 & 6 & 6 \\
\hline \multicolumn{2}{|c|}{ e (dist. entre treliças) } & 3 & 3 & 3 & 3 & 3 & 3 & 4 & 3 & 3 & 3 & 4 \\
\hline \multicolumn{13}{|c|}{ MÉTODO UNDERWOOD (2000) } \\
\hline \multicolumn{2}{|c|}{ Compressão máx $_{\text {mN) }}$} & 65,9 & 68,3 & 74,0 & 116,8 & 120,7 & 126,5 & 160,3 & 185,3 & 190,8 & 202,5 & 244,6 \\
\hline \multirow{6}{*}{$F_{d}(\mathbf{k N})$} & $\mathrm{F}_{1 \mathrm{~d}}$ & 2,1 & 2,1 & 2,3 & 3,7 & 3,8 & 4,0 & 5,0 & 5,8 & 6,0 & 6,3 & 7,6 \\
\hline & $\begin{array}{ll}\mathrm{n} & 7\end{array}$ & 9,6 & 10,0 & 10,8 & 17,0 & 17,6 & 18,4 & - & - & - & - & - \\
\hline & n 11 & - & - & - & - & - & - & 36,7 & - & - & - & 56,1 \\
\hline & n 15 & 20,6 & 21,3 & 23,1 & 36,5 & 37,7 & 39,5 & - & 57,9 & 59,6 & 63,3 & - \\
\hline & n 23 & - & - & - & - & - & - & - & - & - & - & 117,2 \\
\hline & n 31 & - & - & - & - & - & - & - & 119,7 & 123,2 & 130,8 & - \\
\hline \multicolumn{13}{|c|}{ MODELOS NUMÉRICOS } \\
\hline \multicolumn{2}{|c|}{ Compressão $_{\text {máx }}(\mathrm{kN})$} & 72,2 & 75,9 & 105,7 & 121,0 & 134,1 & 134,1 & 171,4 & 195,0 & 202,9 & 212,7 & 258,4 \\
\hline \multirow{5}{*}{$\begin{array}{c}\mathbf{N}_{\mathrm{d}, \mathrm{t}}(\mathbf{k N}) \\
\text { Terças }\end{array}$} & $\mathrm{n}=7$ & 12,4 & 16,3 & 23,5 & 13,0 & 15,7 & 18,4 & - & - & - & - & - \\
\hline & $\mathrm{n}=11$ & - & - & - & - & - & - & 19,1 & - & - & - & 59,3 \\
\hline & $\mathrm{n}=15$ & 12,7 & 16,8 & 24,0 & 16,2 & 18,0 & 20,8 & - & 33,2 & 43,1 & 60,0 & - \\
\hline & $n=23$ & - & - & - & - & - & - & - & - & - & - & 59,2 \\
\hline & $\mathrm{n}=31$ & - & - & - & - & - & - & - & 32,0 & 40,9 & 59,6 & - \\
\hline \multicolumn{13}{|c|}{ COMPARATIVO Galpão/Treliça } \\
\hline \multicolumn{2}{|c|}{ Compressão $_{\text {máx }}(\mathrm{kN})$} & 1,10 & 1,11 & 1,43 & 1,04 & $\mathbf{1 , 1 1}$ & 1,06 & $\mathbf{1 , 0 7}$ & 1,05 & 1,06 & 1,05 & 1,06 \\
\hline \multirow{5}{*}{$\mathbf{N}_{\mathrm{d}, \mathrm{t}} / \mathbf{F}_{\mathbf{d}}$} & $\mathrm{n}=7$ & 1,29 & 1,64 & 2,18 & 0,76 & 0,89 & 1,00 & - & - & - & - & - \\
\hline & $\mathrm{n}=11$ & - & - & - & - & - & - & 0,52 & - & - & - & 1,06 \\
\hline & $\mathrm{n}=15$ & 0,62 & $\mathbf{0 , 7 9}$ & 1,04 & 0,44 & 0,48 & 0,53 & - & 0,57 & 0,72 & 0,95 & - \\
\hline & $n=23$ & - & - & - & - & - & - & - & - & - & - & 0,51 \\
\hline & $\mathrm{n}=31$ & - & - & - & - & - & - & - & 0,27 & 0,33 & 0,46 & - \\
\hline
\end{tabular}


Tabela 5.24 - Comparativo entre o método do SABS 0163 e os modelos tridimensionais (PRATT C50 telha metálica)

\begin{tabular}{|c|c|c|c|c|c|c|c|c|c|c|c|c|}
\hline \multicolumn{13}{|c|}{ GEOMETRIAS DAS TRELIÇAS } \\
\hline \multicolumn{2}{|c|}{ L (vão) } & 12 & 12 & 12 & 18 & 18 & 18 & 18 & 24 & 24 & 24 & 24 \\
\hline \multicolumn{2}{|c|}{ h (altura central) } & 1,5 & 1,5 & 1,5 & 2,25 & 2,25 & 2,25 & 2,25 & 3 & 3 & 3 & 3 \\
\hline \multicolumn{2}{|c|}{ z (altura do pilar) } & 3 & 4 & 6 & 3 & 4 & 6 & 6 & 3 & 4 & 6 & 6 \\
\hline \multicolumn{2}{|c|}{ e (dist. entre treliças) } & 3 & 3 & 3 & 3 & 3 & 3 & 4 & 3 & 3 & 3 & 4 \\
\hline \multicolumn{13}{|c|}{ MÉTODO SABS 0163} \\
\hline \multicolumn{2}{|c|}{ Compressão $_{\text {perm }}(\mathrm{kN})$} & \multicolumn{3}{|c|}{20,1} & \multicolumn{3}{|c|}{41,3} & 46,7 & \multicolumn{3}{|c|}{86,0} & 89,4 \\
\hline \multirow{6}{*}{$F_{d}(k N)$} & $\mathrm{F}_{1 \mathrm{~d}}$ & \multicolumn{3}{|c|}{0,5} & \multicolumn{3}{|c|}{0,7} & 0,8 & \multicolumn{3}{|c|}{1,1} & 1,1 \\
\hline & $\begin{array}{ll}\mathrm{n} & 7\end{array}$ & 2,3 & 2,3 & 2,3 & 3,2 & 3,2 & 3,2 & - & - & - & - & - \\
\hline & n 11 & - & - & - & - & - & - & 5,7 & - & - & - & 8,2 \\
\hline & n 15 & 5,0 & 6,9 & 5,0 & 6,9 & 6,9 & 6,9 & - & 10,8 & 10,8 & 10,8 & - \\
\hline & n 23 & - & - & - & - & - & - & - & - & - & - & 17,1 \\
\hline & n 31 & - & - & - & - & - & - & - & 22,2 & 22,2 & 22,2 & - \\
\hline \multicolumn{13}{|c|}{ MODELOS NUMÉRICOS } \\
\hline \multicolumn{2}{|c|}{ Compressão $_{\text {máx }}(\mathrm{kN})$} & 72,2 & 75,9 & 105,7 & 121,0 & 134,1 & 134,1 & 171,4 & 195,0 & 202,9 & 212,7 & 258,4 \\
\hline \multirow{5}{*}{$\begin{array}{c}\mathbf{N}_{\mathrm{d}, \mathrm{t}}(\mathbf{k N}) \\
\text { Terças }\end{array}$} & $\mathrm{n}=7$ & 12,4 & 16,3 & 23,5 & 13,0 & 15,7 & 18,4 & - & - & & & - \\
\hline & $\mathrm{n}=11$ & - & - & - & - & - & - & 19,1 & - & - & - & 59,3 \\
\hline & $\mathrm{n}=15$ & 12,7 & 16,8 & 24,0 & 16,2 & 18,0 & 20,8 & - & 33,2 & 43,1 & 60,0 & - \\
\hline & $\mathrm{n}=23$ & - & - & - & - & - & - & - & - & - & - & 59,2 \\
\hline & $\mathrm{n}=31$ & - & - & - & - & - & - & - & 32,0 & 40,9 & 59,6 & - \\
\hline \multicolumn{13}{|c|}{ COMPARATIVO Galpão/Treliça } \\
\hline \multirow{5}{*}{$\mathbf{N}_{\mathrm{d}, \mathrm{t}} / \mathbf{F}_{\mathbf{d}}$} & $\mathrm{n}=7$ & 5,29 & 6,95 & 10,02 & 4,05 & 4,89 & 5,73 & - & - & - & & - \\
\hline & $\mathrm{n}=11$ & - & - & - & - & - & - & 3,35 & - & - & - & 7,24 \\
\hline & $\mathrm{n}=15$ & 2,53 & 2,44 & 4,78 & 2,35 & 2,62 & 3,02 & - & 3,09 & 4,01 & 5,58 & - \\
\hline & $n=23$ & - & - & - & - & - & - & - & - & - & - & 3,45 \\
\hline & $\mathrm{n}=31$ & - & - & - & - & - & - & - & 1,44 & 1,84 & 2,68 & - \\
\hline
\end{tabular}


Gráfico 26 - Linhas comparativas dos métodos (PRATT C50 telha metálica)

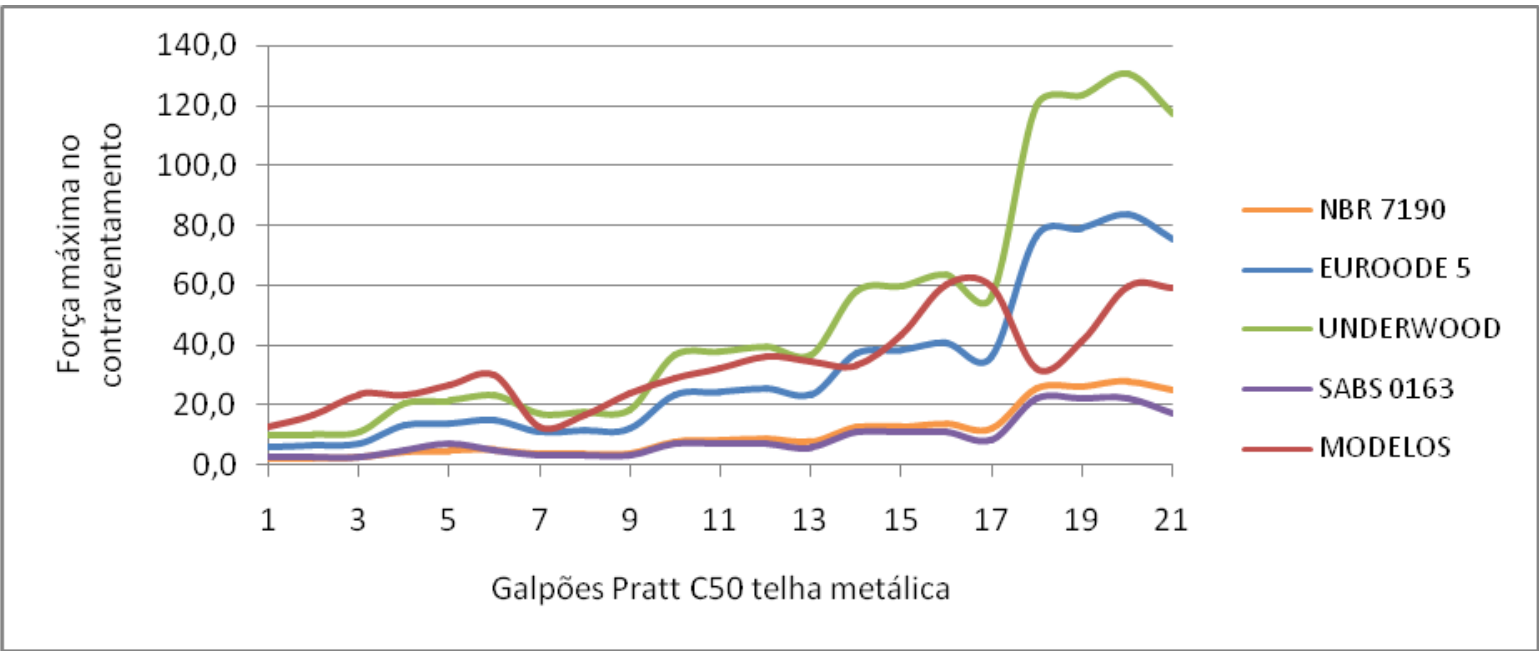

Gráfico 27 - Colunas comparativas do método NBR 7190 (PRATT C50 telha metálica)

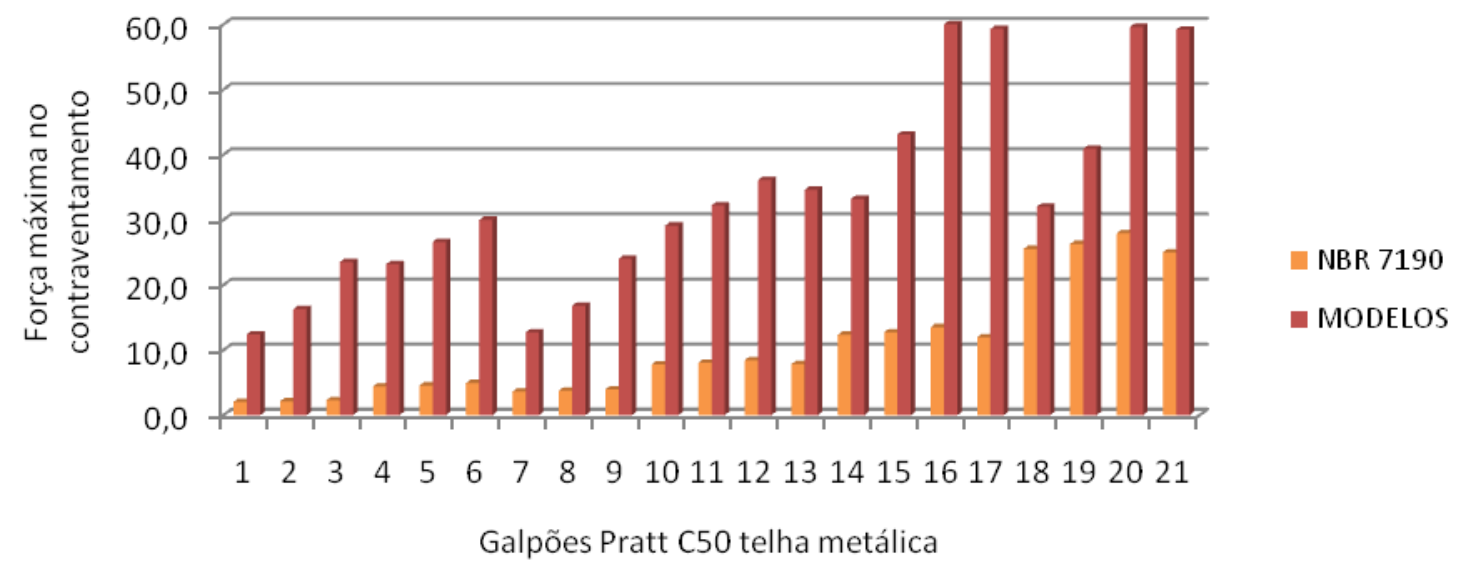

Gráfico 28 - Colunas comparativas do método EUROCODE 5 (PRATT C50 telha metálica)

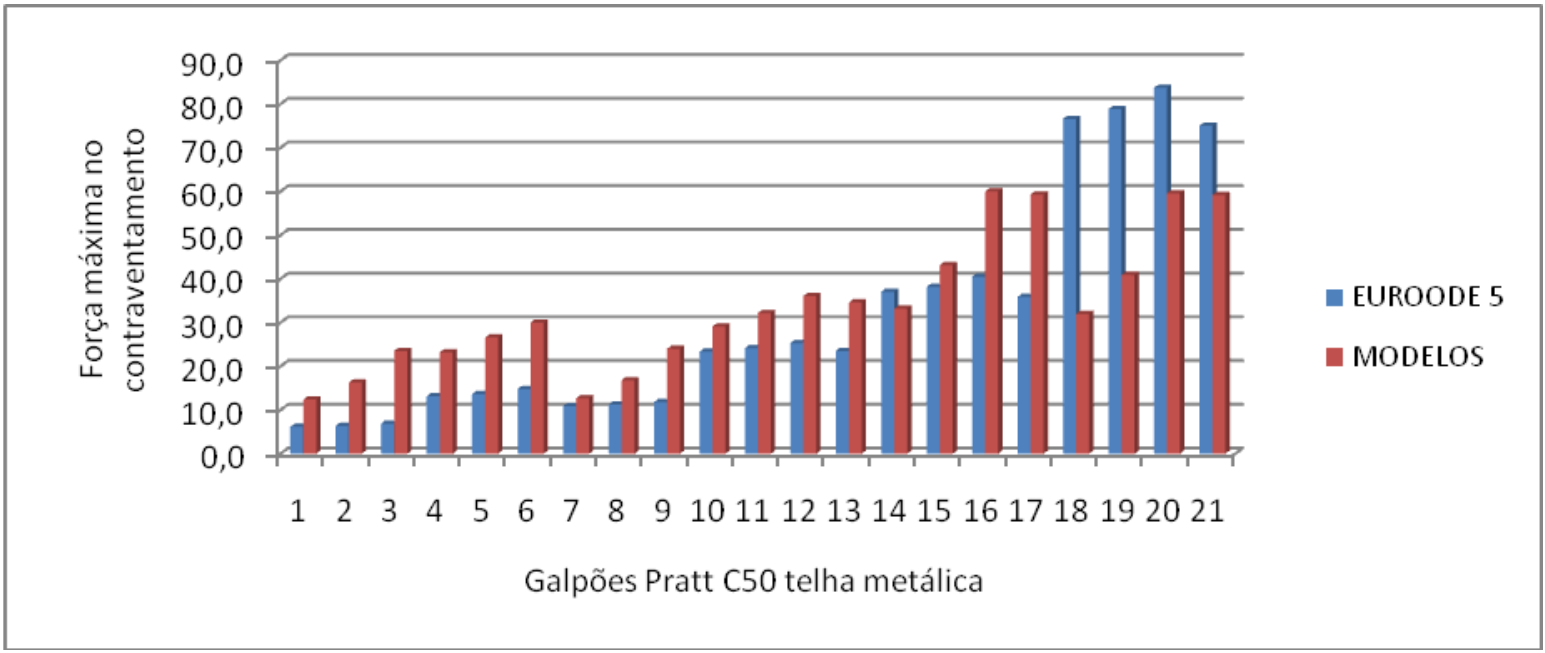


Gráfico 29 - Colunas comparativas do método UNDERWOOD (PRATT C50 telha metálica)

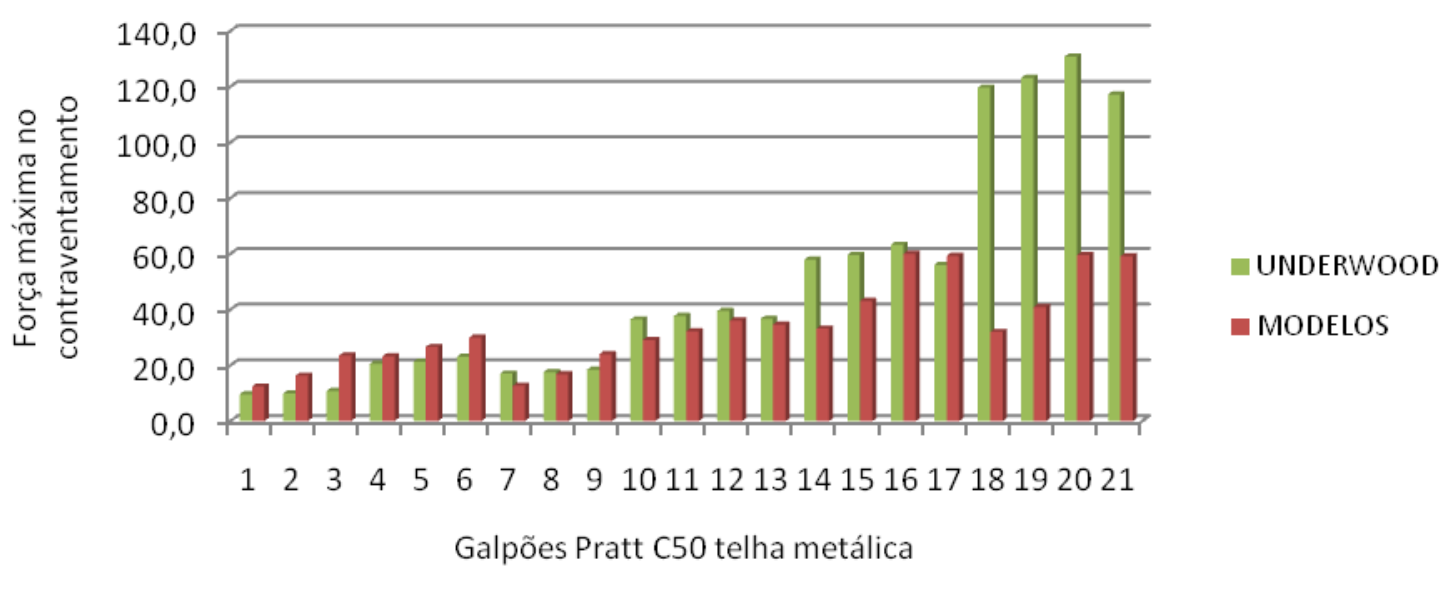

Gráfico 30 - Colunas comparativas do método SABS 0163 (PRATT C50 telha metálica)

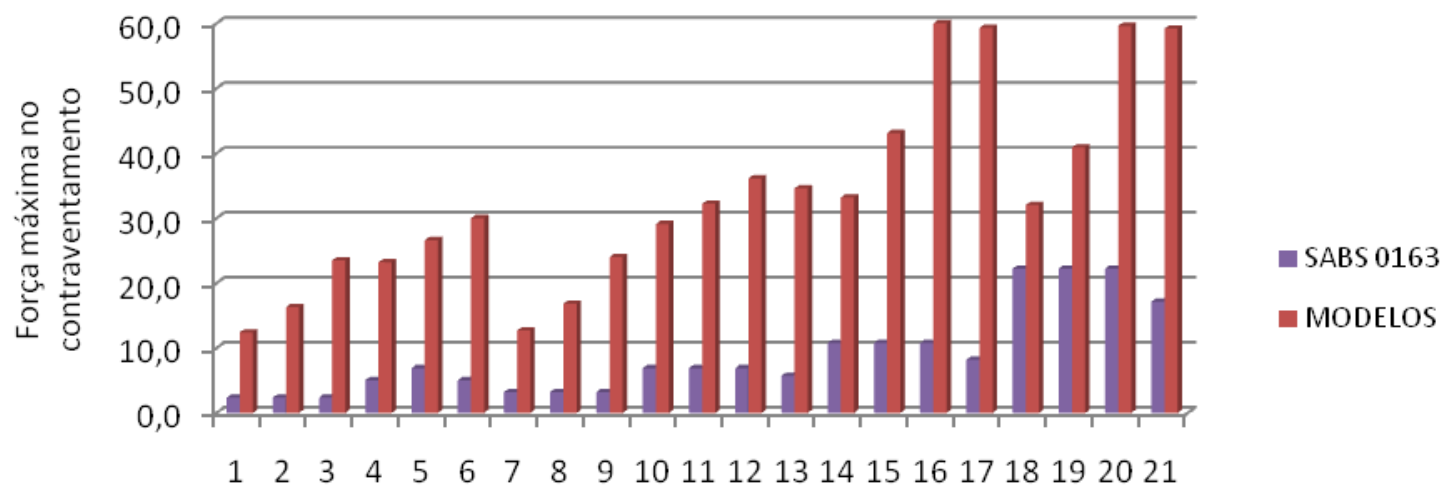

Galpões Pratt C50 telha metálica 


\subsection{Treliças PRATT C50 telha cerâmica}

Tabela 5.25 - Comparativo entre o método da NBR 7190 (1997) e os modelos tridimensionais (PRATT C50 telha cerâmica)

\begin{tabular}{|c|c|c|c|c|c|c|c|}
\hline \multicolumn{8}{|c|}{ GEOMETRIAS DAS TRELIÇAS } \\
\hline \multicolumn{2}{|c|}{ L (vão) } & 12 & 12 & 12 & 18 & 18 & 18 \\
\hline \multicolumn{2}{|c|}{ h (altura central) } & 1,5 & 1,5 & 1,5 & 2,25 & 2,25 & 2,25 \\
\hline \multicolumn{2}{|c|}{ z (altura do pilar) } & 3 & 4 & 6 & 3 & 4 & 6 \\
\hline \multicolumn{2}{|c|}{ e (dist. entre treliças) } & 3 & 3 & 3 & 3 & 3 & 3 \\
\hline \multicolumn{8}{|c|}{ MÉTODO NBR 7190 (1997) } \\
\hline \multicolumn{2}{|c|}{ Compressão máx $_{\text {(kN) }}$} & 155,2 & 157,6 & 163,5 & 278,1 & 282,0 & 287,7 \\
\hline \multirow{3}{*}{$\mathbf{F}_{\mathrm{d}}(\mathbf{k N})$} & $\mathrm{F}_{1 \mathrm{~d}}$ & 1,0 & 1,1 & 1,1 & 1,9 & 1,9 & 1,9 \\
\hline & & 4,8 & 4,9 & 5,1 & 8,7 & 8,8 & 9,0 \\
\hline & 15 & 10,3 & 10,5 & 10,9 & 18,5 & 18,8 & 19,2 \\
\hline \multicolumn{8}{|c|}{ MODELOS NUMÉRICOS } \\
\hline \multicolumn{2}{|c|}{ Compressão $_{\text {máx }}(\mathrm{kN})$} & 167,7 & 171,4 & 176,0 & 290,3 & 296,2 & 303,6 \\
\hline $\mathbf{N}_{\mathrm{d}, \mathrm{t}}(\mathrm{kN})$ & $n=$ & 12,4 & 16,3 & 23,5 & 13,9 & 14,3 & 15,9 \\
\hline Terças & $\mathrm{n}=15$ & 12,7 & 16,8 & 24,0 & 10,4 & 12,1 & 18,2 \\
\hline \multicolumn{8}{|c|}{ COMPARATIVO Galpão/Treliça } \\
\hline \multicolumn{2}{|c|}{ Compressão $_{\text {máx }}(\mathrm{kN})$} & 1,08 & 1,09 & 1,08 & 1,04 & 1,05 & 1,06 \\
\hline \multirow{2}{*}{$\mathbf{N}_{\mathrm{d}, \mathbf{t}} / \mathbf{F}_{\mathbf{d}}$} & $\mathrm{n}=7$ & 2,57 & 3,32 & 4,62 & 1,61 & 1,63 & 1,78 \\
\hline & $\mathrm{n}=15$ & 1,23 & 1,60 & 2,20 & 0,56 & 0,64 & 0,95 \\
\hline
\end{tabular}

Tabela 5.26 - Comparativo entre o método do EUROCODE 5 e os modelos tridimensionais (cerâmica)

\begin{tabular}{|c|c|c|c|c|c|c|c|}
\hline \multicolumn{8}{|c|}{ GEOMETRIAS DAS TRELIÇAS } \\
\hline \multicolumn{2}{|c|}{ L (vão) } & 12 & 12 & 12 & 18 & 18 & 18 \\
\hline \multicolumn{2}{|c|}{ h (altura central) } & 1,5 & 1,5 & 1,5 & 2,25 & 2,25 & 2,25 \\
\hline \multicolumn{2}{|c|}{ z (altura do pilar) } & 3 & 4 & 6 & 3 & 4 & 6 \\
\hline \multicolumn{2}{|c|}{ e (dist. entre treliças) } & 3 & 3 & 3 & 3 & 3 & 3 \\
\hline \multicolumn{8}{|c|}{ MÉTODO EUROCODE 5} \\
\hline \multicolumn{2}{|c|}{ Compressão máx $(k N)$} & 155,2 & 157,6 & 163,5 & 278,1 & 282,0 & 287,7 \\
\hline \multirow{3}{*}{$F_{d}(\mathbf{k N})$} & $F_{1 d}$ & 3,1 & 3,2 & 3,3 & 5,6 & 5,6 & 5,8 \\
\hline & & 14,5 & 14,7 & 15,3 & 26,0 & 26,3 & 26,9 \\
\hline & 15 & 31,0 & 31,5 & 32,7 & 55,6 & 56,4 & 57,5 \\
\hline \multicolumn{8}{|c|}{ MODELOS NUMÉRICOS } \\
\hline \multicolumn{2}{|c|}{ Compressão máx $(\mathrm{kN})$} & 167,7 & 171,4 & 176,0 & 290,3 & 296,2 & 303,6 \\
\hline \multirow{2}{*}{$\begin{array}{c}\mathbf{N}_{\mathbf{d}, \mathbf{t}}(\mathbf{k N}) \\
\text { Terças } \\
\end{array}$} & $\mathrm{n}=$ & 12,4 & 16,3 & 23,5 & 13,9 & 14,3 & 15,9 \\
\hline & $\mathrm{n}=15$ & 12,7 & 16,8 & 24,0 & 10,4 & 12,1 & 18,2 \\
\hline \multicolumn{8}{|c|}{ COMPARATIVO Galpão/Treliça } \\
\hline \multicolumn{2}{|c|}{ Compressão $_{\text {máx }}(\mathrm{kN})$} & 1,08 & 1,09 & 1,08 & 1,04 & 1,05 & 1,06 \\
\hline \multirow{2}{*}{$\mathbf{N}_{\mathbf{d}, \mathbf{t}} / \mathbf{F}_{\mathbf{d}}$} & $\mathrm{n}=$ & $\mathbf{0 , 8 6}$ & 1,11 & 1,54 & 0,54 & 0,54 & 0,59 \\
\hline & $\mathrm{n}=$ & 0,41 & 0,53 & $\mathbf{0 , 7 3}$ & $\mathbf{0 , 1 9}$ & 0,21 & 0,32 \\
\hline
\end{tabular}


Tabela 5.27 - Comparativo entre o método de UNDERWOOD (2000) e os modelos tridimensionais (PRATT C50 telha cerâmica)

\begin{tabular}{|c|c|c|c|c|c|c|c|}
\hline \multicolumn{8}{|c|}{ GEOMETRIAS DAS TRELIÇAS } \\
\hline \multicolumn{2}{|c|}{ L (vão) } & 12 & 12 & 12 & 18 & 18 & 18 \\
\hline \multicolumn{2}{|c|}{ h (altura central) } & 1,5 & 1,5 & 1,5 & 2,25 & 2,25 & 2,25 \\
\hline \multicolumn{2}{|c|}{ z (altura do pilar) } & 3 & 4 & 6 & 3 & 4 & 6 \\
\hline \multicolumn{2}{|c|}{ e (dist. entre treliças) } & 3 & 3 & 3 & 3 & 3 & 3 \\
\hline \multicolumn{8}{|c|}{ MÉTODO UNDERWOOD (2000) } \\
\hline \multicolumn{2}{|c|}{ Compressão $_{\text {máx }}(\mathrm{kN})$} & 155,2 & 157,6 & 163,5 & 278,1 & 282,0 & 287,7 \\
\hline \multirow{3}{*}{$\mathbf{F}_{\mathrm{d}}(\mathbf{k N})$} & $\mathrm{F}_{1 \mathrm{~d}}$ & 4,9 & 4,9 & 5,1 & 8,7 & 8,8 & 9,0 \\
\hline & & 22,6 & 23,0 & 23,8 & 40,6 & 41,1 & 42,0 \\
\hline & 15 & 48,5 & 49,3 & 51,1 & 86,9 & 88,1 & 89,9 \\
\hline \multicolumn{8}{|c|}{ MODELOS NUMÉRICOS } \\
\hline \multicolumn{2}{|c|}{ Compressão máx $_{\text {(kN) }}$} & 167,7 & 171,4 & 176,0 & 290,3 & 296,2 & 303,6 \\
\hline $\mathbf{N}_{\mathrm{d}, \mathrm{t}}(\mathbf{k N})$ & $\mathrm{n}=$ & 12,4 & 16,3 & 23,5 & 13,9 & 14,3 & 15,9 \\
\hline Terças & $\mathrm{n}=15$ & 12,7 & 16,8 & 24,0 & 10,4 & 12,1 & 18,2 \\
\hline \multicolumn{8}{|c|}{ COMPARATIVO Galpão/Treliça } \\
\hline \multicolumn{2}{|c|}{ Compressãomáx $(\mathrm{kN})$} & 1,08 & 1,09 & 1,08 & 1,04 & 1,05 & 1,06 \\
\hline \multirow{2}{*}{$\mathbf{N}_{\mathrm{d}, \mathrm{t}} / \mathbf{F}_{\mathbf{d}}$} & $\mathrm{n}=7$ & 0,55 & 0,71 & 0,99 & 0,34 & 0,35 & 0,38 \\
\hline & $\mathrm{n}=15$ & 0,26 & 0,34 & 0,47 & $\mathbf{0 , 1 2}$ & 0,14 & $\mathbf{0 , 2 0}$ \\
\hline
\end{tabular}

Tabela 5.28 - Comparativo entre o método do SABS 0163 e os modelos tridimensionais (PRATT C50 telha cerâmica)

\begin{tabular}{|c|c|c|c|c|c|c|c|}
\hline \multicolumn{8}{|c|}{ GEOMETRIAS DAS TRELIÇAS } \\
\hline \multicolumn{2}{|c|}{ L (vão) } & 12 & 12 & 12 & 18 & 18 & 18 \\
\hline \multicolumn{2}{|c|}{ h (altura central) } & 1,5 & 1,5 & 1,5 & 2,25 & 2,25 & 2,25 \\
\hline \multicolumn{2}{|c|}{ z (altura do pilar) } & 3 & 4 & 6 & 3 & 4 & 6 \\
\hline \multicolumn{2}{|c|}{ e (dist. entre treliças) } & 3 & 3 & 3 & 3 & 3 & 3 \\
\hline \multicolumn{8}{|c|}{ MÉTODO SABS 0163} \\
\hline \multicolumn{2}{|c|}{ Compressão $_{\text {perm }}(\mathrm{kN})$} & \multicolumn{3}{|c|}{109,5} & \multicolumn{3}{|c|}{202,5} \\
\hline \multirow{3}{*}{$\mathbf{F}_{\mathbf{d}}(\mathbf{k N})$} & $\mathrm{F}_{1 \mathrm{~d}}$ & \multicolumn{3}{|c|}{2,7} & \multicolumn{3}{|c|}{3,4} \\
\hline & 7 & 12,8 & 12,8 & 12,8 & 15,8 & 15,8 & 15,8 \\
\hline & 15 & 27,4 & 33,8 & 27,4 & 33,8 & 33,8 & 33,8 \\
\hline \multicolumn{8}{|c|}{ MODELOS NUMÉRICOS } \\
\hline \multicolumn{2}{|c|}{ Compressãomáx $_{\text {maN) }}$} & 167,7 & 171,4 & 176,0 & 290,3 & 296,2 & 303,6 \\
\hline $\mathbf{N}_{\mathrm{d}, \mathrm{t}}(\mathrm{kN})$ & $\mathrm{n}=$ & 12,4 & 16,3 & 23,5 & 13,9 & 14,3 & 15,9 \\
\hline Terças & $\mathrm{n}=15$ & 12,7 & 16,8 & 24,0 & 10,4 & 12,1 & 18,2 \\
\hline \multicolumn{8}{|c|}{ COMPARATIVO Galpão/Treliça } \\
\hline \multirow{2}{*}{$\mathbf{N}_{\mathrm{d}, \mathrm{t}} / \mathbf{F}_{\mathbf{d}}$} & $\mathrm{n}=7$ & 0,97 & 1,28 & 1,84 & 0,88 & 0,91 & 1,01 \\
\hline & $\mathrm{n}=15$ & 0,46 & $\mathbf{0 , 5 0}$ & 0,88 & 0,31 & 0,36 & 0,54 \\
\hline
\end{tabular}


Gráfico 31 - Linhas comparativas dos métodos (PRATT C50 telha cerâmica)

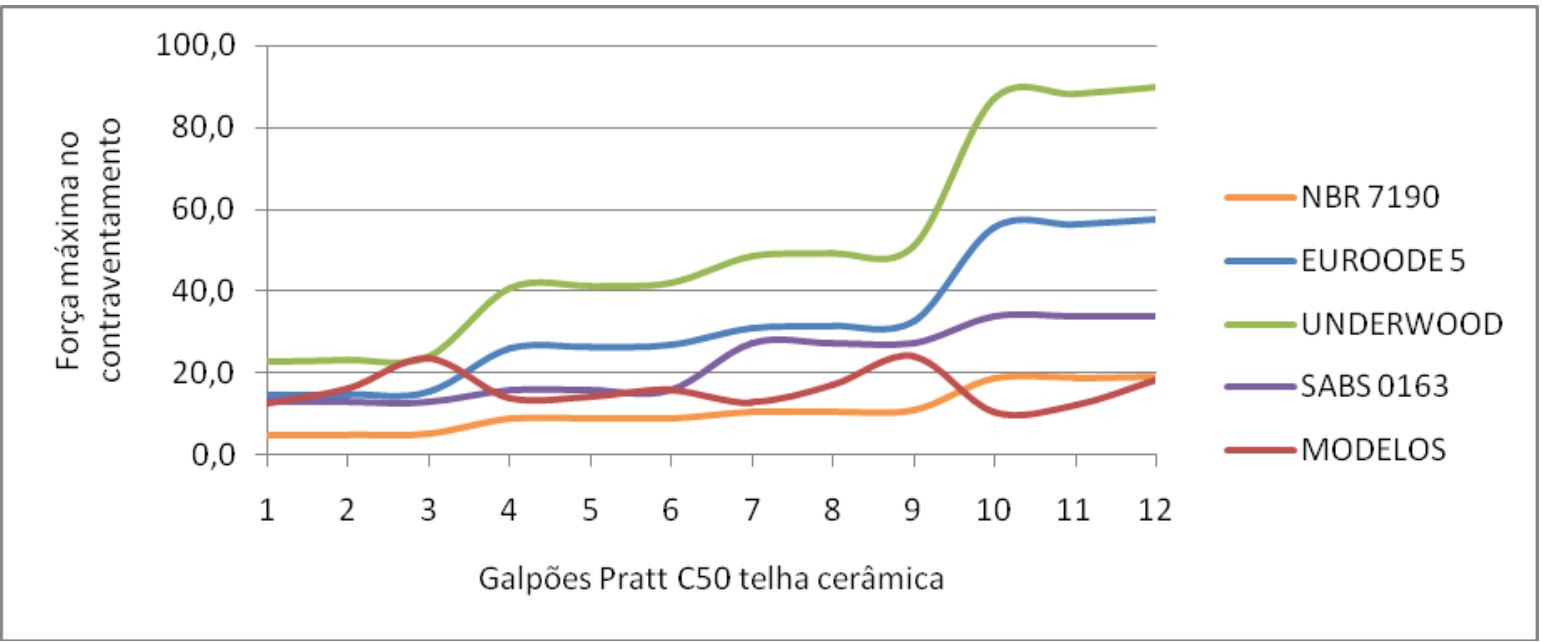

Gráfico 32 - Colunas comparativas do método NBR 7190 (PRATT C50 telha cerâmica)

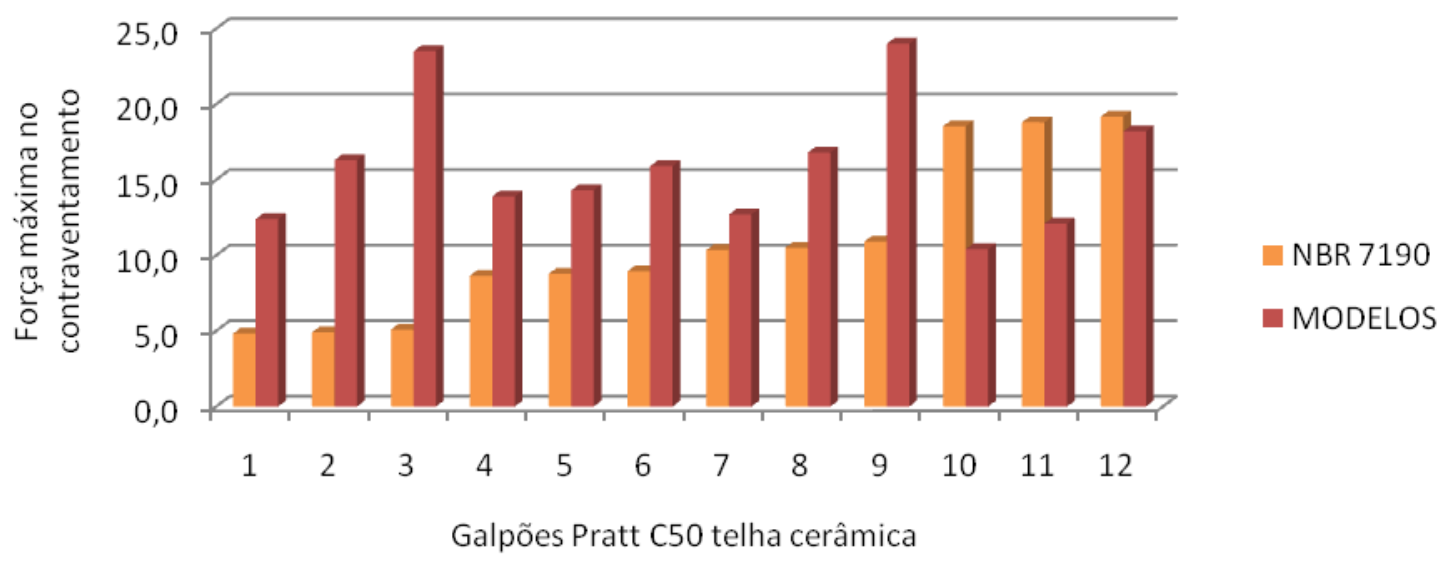

Gráfico 33 - Colunas comparativas do método EUROCODE 5 (PRATT C50 telha cerâmica)

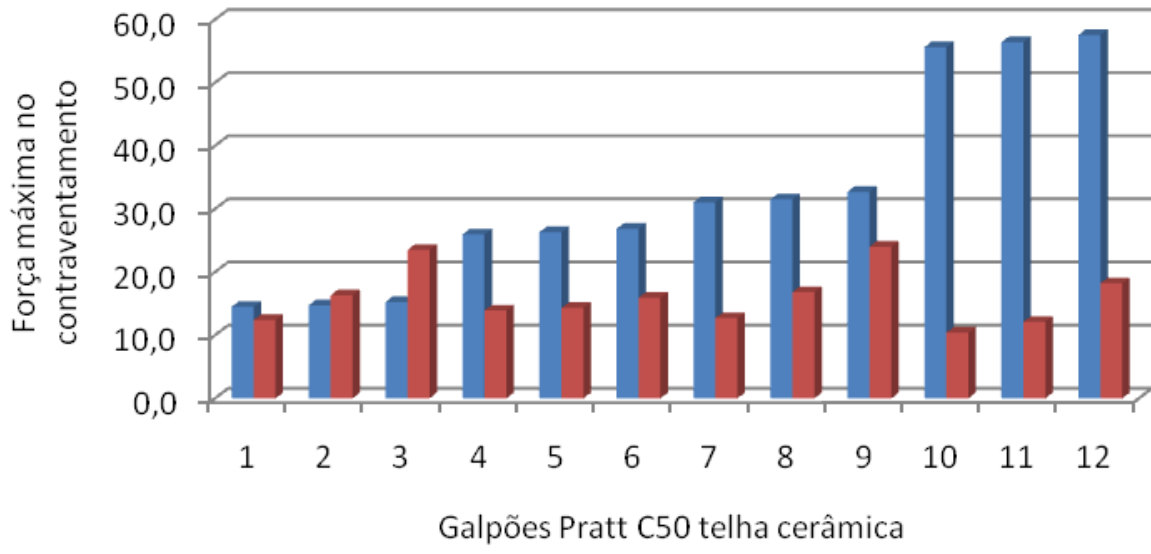

- EUROODE 5

- MODELOS 
Gráfico 34 - Colunas comparativas do método UNDERWOOD (PRATT C50 telha cerâmica)

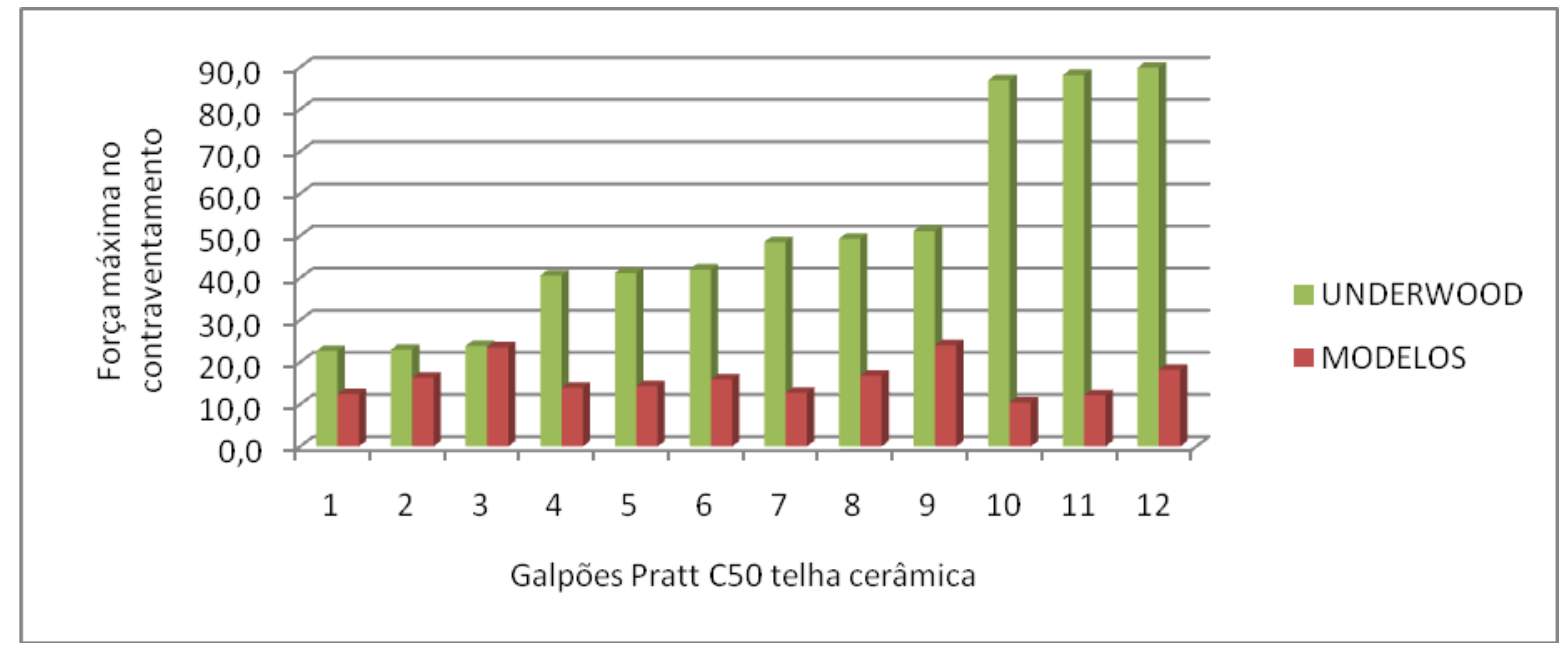

Gráfico 35 - Colunas comparativas do método SABS 0163 (PRATT C50 telha cerâmica)

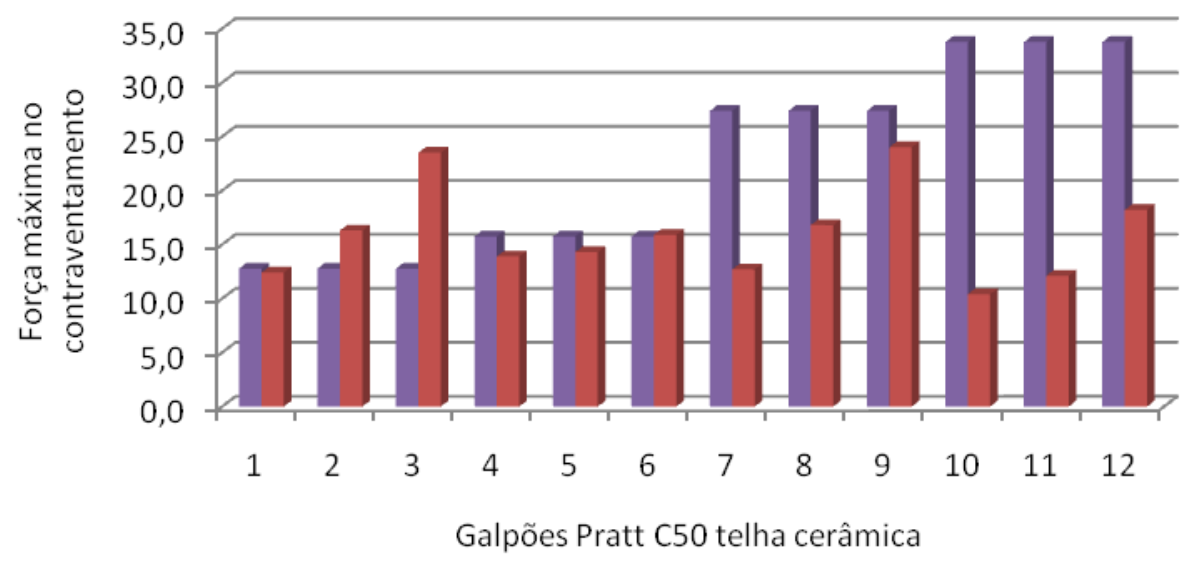

SABS 0163

MODELOS 


\subsection{Comparativo entre tipos de treliças e classes de resistência}

A seguir são comparados os tipos de treliças e classes de madeira.

Tabela 5.29 - Forças de compressão máximas nos banzos das treliças de telha fibrocimento

\begin{tabular}{|c|ccccc|}
\hline Galpão & $\begin{array}{c}\text { HOWE } \\
\text { C50 }\end{array}$ & $\begin{array}{c}\text { PRATT } \\
\text { C50 }\end{array}$ & $\begin{array}{c}\text { HOWE } \\
\text { C30 }\end{array}$ & $\begin{array}{c}\text { PRATT } \\
\text { C30 }\end{array}$ & $\begin{array}{c}\text { CRATT } \\
\text { madeira }\end{array}$ \\
\hline $\mathbf{1}$ & 92,3 & 94,0 & 88,2 & 89,8 & 100,2 \\
\hline $\mathbf{2}$ & 95,9 & 97,7 & 91,7 & 93,5 & 103,6 \\
\hline $\mathbf{3}$ & 100,4 & 102,3 & 96,2 & 98,1 & 107,9 \\
\hline $\mathbf{4}$ & 92,2 & 93,8 & 88,1 & 89,8 & 101,0 \\
\hline $\mathbf{5}$ & 95,8 & 97,5 & 91,7 & 93,5 & 104,5 \\
\hline $\mathbf{6}$ & 92,5 & 94,2 & 91,6 & 97,9 & 101,4 \\
\hline $\mathbf{7}$ & 149,7 & 152,5 & 156,8 & 164,6 & 157,3 \\
\hline $\mathbf{8}$ & 155,3 & 158,2 & 166,7 & 170,4 & 162,8 \\
\hline $\mathbf{9}$ & 162,4 & 165,4 & 173,8 & 177,7 & 169,7 \\
\hline $\mathbf{1 0}$ & 148,2 & 153,0 & 159,0 & 165,1 & 164,2 \\
\hline $\mathbf{1 1}$ & 153,8 & 158,8 & 164,7 & 170,9 & 169,9 \\
\hline $\mathbf{1 2}$ & 160,9 & 166,1 & 171,7 & 180,5 & 177,1 \\
\hline $\mathbf{1 3}$ & 209,2 & 216,6 & 214,3 & 224,4 & 233,8 \\
\hline $\mathbf{1 4}$ & 238,1 & 246,0 & 241,2 & 248,9 & 263,5 \\
\hline $\mathbf{1 5}$ & 245,6 & 254,0 & 248,6 & 256,9 & 271,3 \\
\hline $\mathbf{1 6}$ & 254,0 & 263,2 & 257,0 & 266,2 & 280,2 \\
\hline $\mathbf{1 7}$ & 307,3 & 318,1 & 314,9 & 324,9 & 343,2 \\
\hline $\mathbf{1 8}$ & 230,6 & 243,2 & 239,6 & 245,7 & 261,0 \\
\hline $\mathbf{1 9}$ & 244,0 & 251,3 & 247,2 & 253,8 & 269,0 \\
\hline $\mathbf{2 0}$ & 236,4 & 243,2 & 239,6 & 245,7 & 261,1 \\
\hline $\mathbf{2 1}$ & 293,4 & 296,4 & 295,2 & 298,4 & 322,0 \\
\hline & & & & & \\
\hline
\end{tabular}

Gráfico 36 - Colunas comparativas das forças $(\mathrm{kN})$ de compressão máximas nos banzos

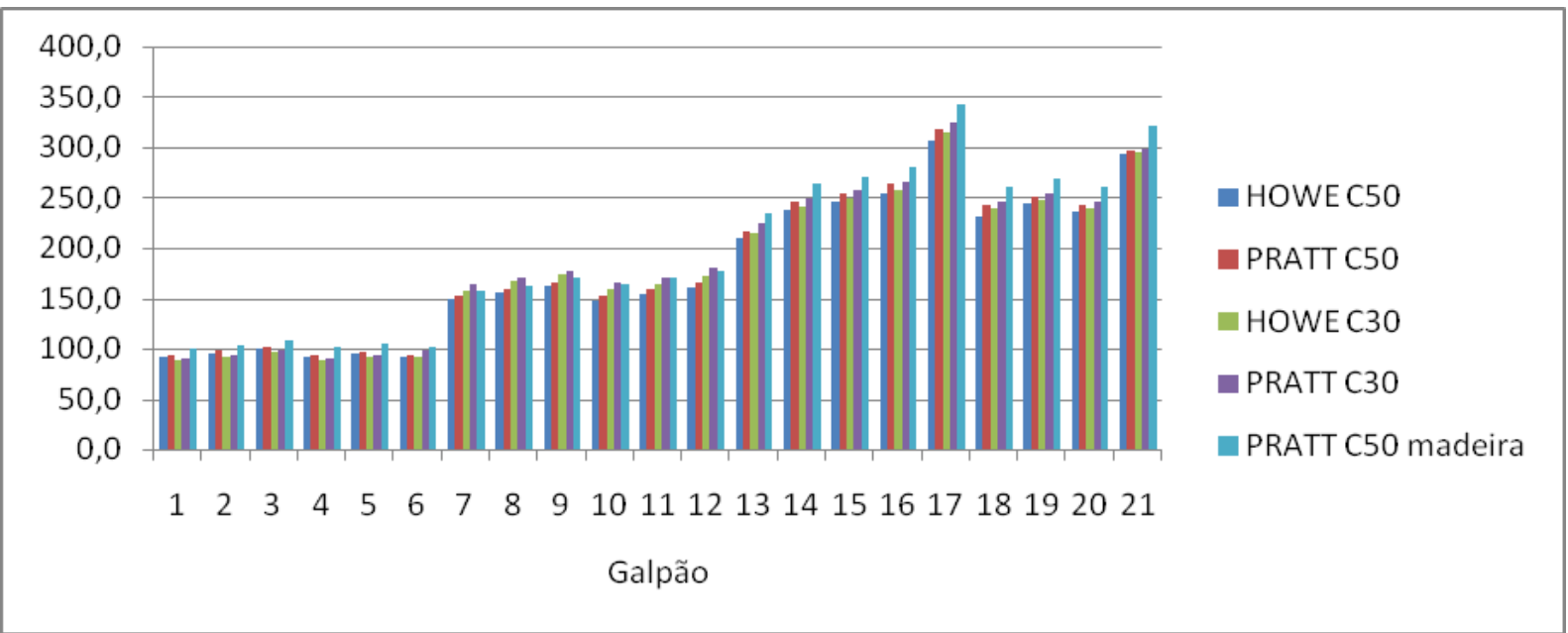


Tabela 5.30 - Forças de compressão máximas no contraventamento

\begin{tabular}{|c|ccccc|}
\hline Galpão & $\begin{array}{c}\text { HOWE } \\
\text { C50 }\end{array}$ & $\begin{array}{c}\text { PRATT } \\
\text { C50 }\end{array}$ & $\begin{array}{c}\text { HOWE } \\
\text { C30 }\end{array}$ & $\begin{array}{c}\text { PRATT } \\
\text { C30 }\end{array}$ & $\begin{array}{c}\text { PRATT } \\
\text { C50 } \\
\text { madeira }\end{array}$ \\
\hline $\mathbf{1}$ & 12,4 & 12,4 & 12,4 & 12,4 & 12,8 \\
\hline $\mathbf{2}$ & 16,3 & 16,3 & 16,3 & 16,3 & 13,3 \\
\hline $\mathbf{3}$ & 23,5 & 23,5 & 23,5 & 23,5 & 15,8 \\
\hline $\mathbf{4}$ & 12,8 & 12,8 & 12,8 & 12,8 & 12,4 \\
\hline $\mathbf{5}$ & 16,9 & 16,9 & 16,9 & 16,9 & 13,5 \\
\hline $\mathbf{6}$ & 24,0 & 24,0 & 23,8 & 23,8 & 16,1 \\
\hline $\mathbf{7}$ & 16,1 & 9,7 & 16,7 & 8,4 & 12,5 \\
\hline $\mathbf{8}$ & 16,7 & 12,4 & 17,4 & 11,0 & 12,9 \\
\hline $\mathbf{9}$ & 17,5 & 15,4 & 18,2 & 16,1 & 13,4 \\
\hline $\mathbf{1 0}$ & 14,8 & 11,9 & 15,7 & 9,4 & 17,0 \\
\hline $\mathbf{1 1}$ & 15,4 & 13,7 & 16,3 & 12,1 & 17,4 \\
\hline $\mathbf{1 2}$ & 17,0 & 17,0 & 18,7 & 18,4 & 18,0 \\
\hline $\mathbf{1 3}$ & 19,1 & 18,5 & 19,9 & 19,7 & 13,7 \\
\hline $\mathbf{1 4}$ & 33,2 & 33,3 & 33,2 & 33,4 & 24,2 \\
\hline $\mathbf{1 5}$ & 43,1 & 43,5 & 43,5 & 43,6 & 29,3 \\
\hline $\mathbf{1 6}$ & 60,0 & 60,0 & 60,0 & 60,0 & 37,3 \\
\hline $\mathbf{1 7}$ & 59,3 & 59,3 & 59,3 & 59,4 & 35,7 \\
\hline $\mathbf{1 8}$ & 31,4 & 32,1 & 31,9 & 32,2 & 24,3 \\
\hline $\mathbf{1 9}$ & 35,5 & 41,0 & 40,9 & 41,1 & 28,8 \\
\hline $\mathbf{2 0}$ & 59,6 & 59,6 & 59,6 & 59,7 & 38,5 \\
\hline $\mathbf{2 1}$ & 59,2 & 59,2 & 59,2 & 59,2 & 36,9 \\
\hline & & & & & \\
\hline
\end{tabular}

Gráfico 37 - Colunas comparativas das forças $(\mathrm{kN})$ de compressão máximas no contraventamento

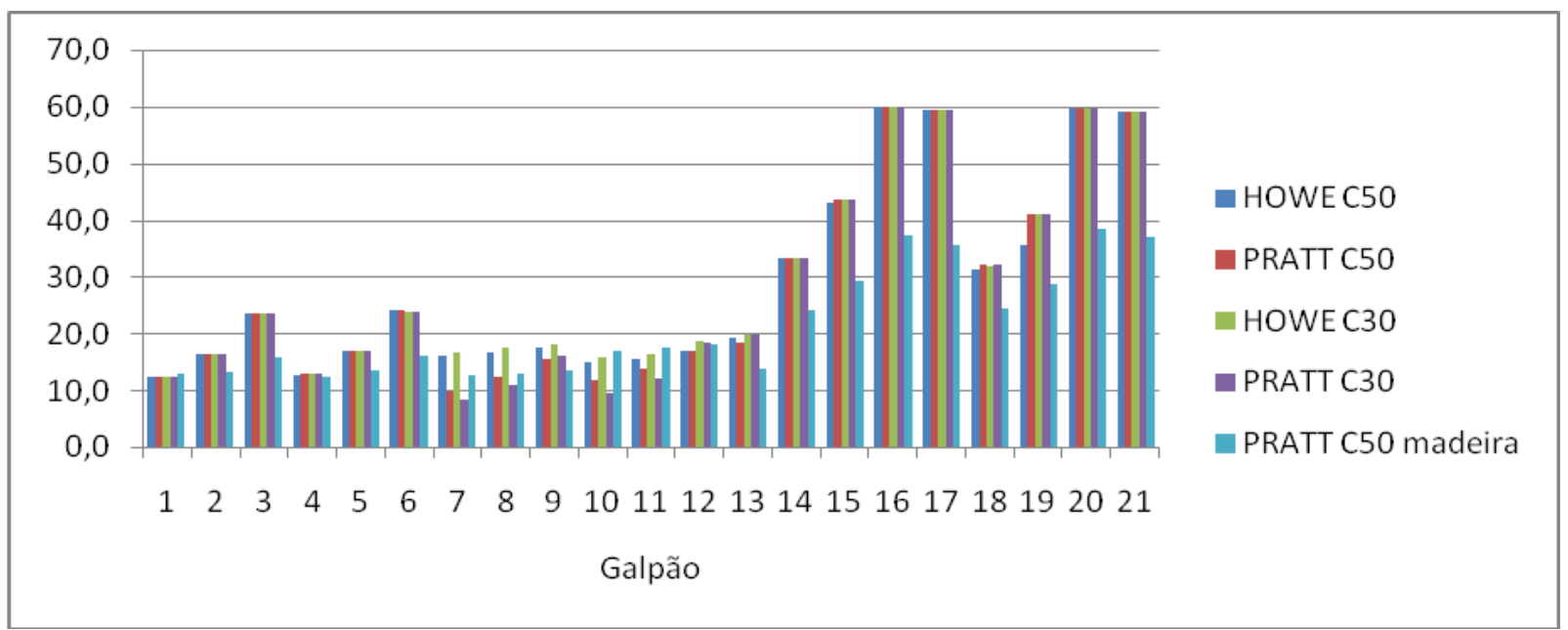




\subsection{Comparativo entre tipos de telhas}

Os gráficos comparam as forças máximas de compressão nas treliças e no contraventamento dos galpões.

Tabela 5.31 - Forças de compressão máximas nos banzos das treliças Pratt C50

\begin{tabular}{|c|c|c|c|}
\hline Galpão & METÁLICA & FIBROCIMENTO & CERÂMICA \\
\hline 1 & 71,0 & 94,0 & 167,6 \\
\hline 2 & 75,9 & 97,7 & 167,5 \\
\hline 3 & 80,4 & 102,3 & 175,9 \\
\hline 4 & 72,2 & 93,8 & 167,7 \\
\hline 5 & 75,9 & 97,5 & 171,4 \\
\hline 6 & 105,7 & 94,2 & 176,0 \\
\hline 7 & 121,0 & 152,5 & 288,1 \\
\hline 8 & 126,7 & 158,2 & 293,9 \\
\hline 9 & 134,0 & 165,4 & 301,2 \\
\hline 10 & 121,0 & 153,0 & 290,3 \\
\hline 11 & 126,8 & 158,8 & 296,2 \\
\hline 12 & 134,1 & 166,1 & 303,6 \\
\hline 13 & 171,4 & 216,6 & \\
\hline 14 & 195,0 & 246,0 & \\
\hline 15 & 202,9 & 254,0 & \\
\hline 16 & 212,7 & 263,2 & \\
\hline 17 & 258,4 & 318,1 & \\
\hline 18 & 192,1 & 243,2 & \\
\hline 19 & 200,1 & 251,3 & \\
\hline 20 & 192,1 & 243,2 & \\
\hline 21 & 237,6 & 316,3 & \\
\hline
\end{tabular}

Gráfico 38 - Colunas das forças máximas de compressão nos banzos

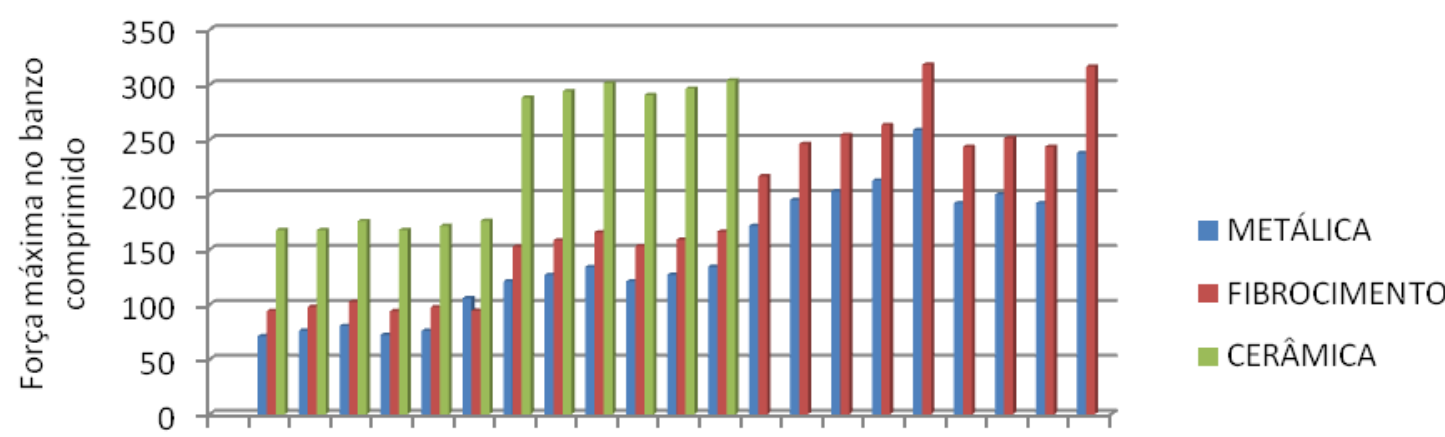

12344567789910111213141516171819202122

Galpões 
Tabela 5.32 - Forças de compressão máximas no contraventamento das treliças Pratt C50

\begin{tabular}{|c|c|c|c|}
\hline Galpão & METÁLICA & FIBROCIMENTO & CERÂMICA \\
\hline 1 & 12,4 & 12,4 & 12,4 \\
\hline 2 & 16,3 & 16,3 & 16,3 \\
\hline 3 & 23,5 & 23,5 & 23,5 \\
\hline 4 & 12,7 & 12,8 & 12,7 \\
\hline 5 & 16,8 & 16,9 & 16,8 \\
\hline 6 & 23,9 & 24,0 & 24,0 \\
\hline 7 & 13,0 & 9,7 & 13,9 \\
\hline 8 & 15,7 & 12,4 & 14,3 \\
\hline 9 & 18,4 & 15,4 & 15,9 \\
\hline 10 & 16,2 & 11,9 & 10,4 \\
\hline 11 & 18,0 & 13,7 & 12,1 \\
\hline 12 & 20,8 & 17,0 & 18,2 \\
\hline 13 & 19,1 & 18,5 & \\
\hline 14 & 33,2 & 33,3 & \\
\hline 15 & 43,1 & 43,5 & \\
\hline 16 & 60,0 & 60,0 & \\
\hline 17 & 59,3 & 59,3 & \\
\hline 18 & 32,0 & 32,1 & \\
\hline 19 & 40,9 & 41,0 & \\
\hline 20 & 59,6 & 59,6 & \\
\hline 21 & 59,2 & 59,2 & \\
\hline
\end{tabular}

Gráfico 39 - Colunas das forças máximas de compressão no contraventamento

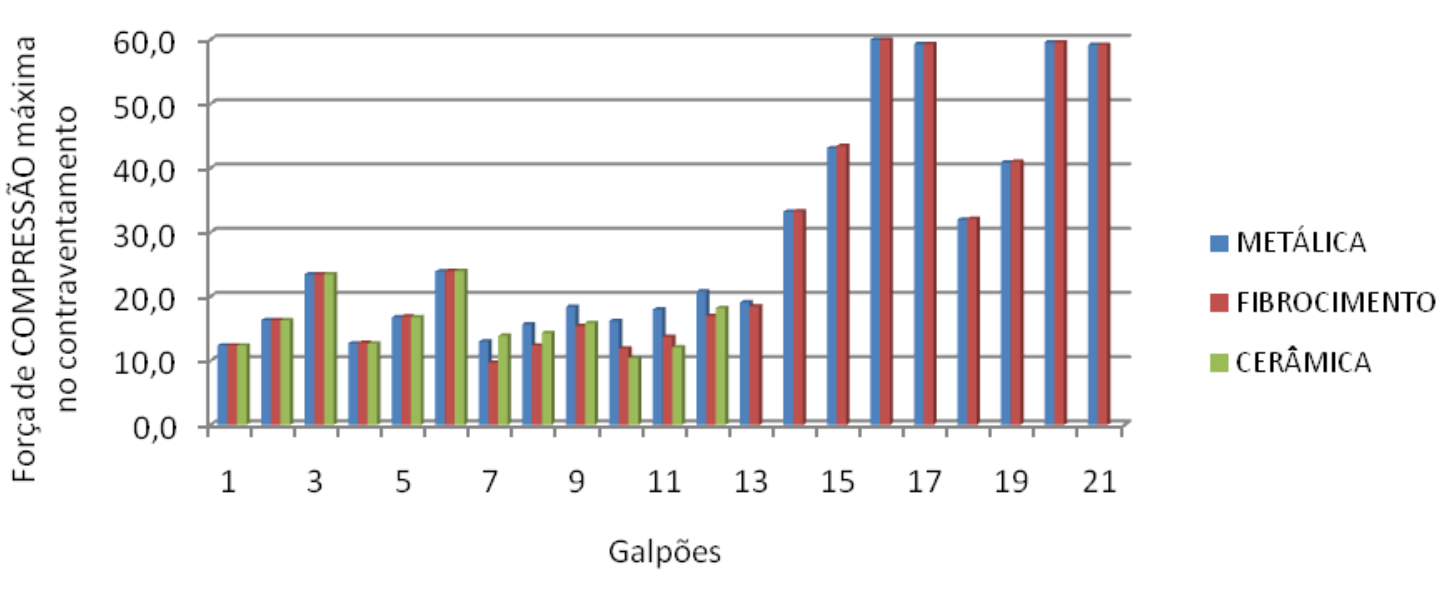


Tabela 5.33 - Forças de tração máximas no contraventamento das treliças Pratt C50

\begin{tabular}{|c|c|c|c|}
\hline Galpão & METÁLICA & FIBROCIMENTO & CERÂMICA \\
\hline 1 & 14,9 & 8,4 & 6,5 \\
\hline 2 & 17,3 & 10,6 & 8,8 \\
\hline 3 & 20,6 & 14,7 & 13,2 \\
\hline 4 & 14,7 & 8,7 & 7,2 \\
\hline 5 & 17,2 & 11,1 & 9,7 \\
\hline 6 & 20,9 & 15,6 & 14,2 \\
\hline 7 & 26,8 & 23,0 & 16,5 \\
\hline 8 & 36,4 & 32,8 & 23,9 \\
\hline 9 & 47,6 & 44,0 & 36,4 \\
\hline 10 & 27,4 & 23,6 & 19,1 \\
\hline 11 & 36,2 & 32,6 & 25,9 \\
\hline 12 & 49,9 & 46,5 & 41,7 \\
\hline 13 & 47,6 & 45,1 & \\
\hline 14 & 31,4 & 29,7 & \\
\hline 15 & 38,6 & 37,1 & \\
\hline 16 & 61,6 & 55,8 & \\
\hline 17 & 63,6 & 56,1 & \\
\hline 18 & 27,7 & 26,6 & \\
\hline 19 & 35,4 & 34,5 & \\
\hline 20 & 60,5 & 55,8 & \\
\hline 21 & 61,4 & 55,4 & \\
\hline
\end{tabular}

Gráfico 40 - Colunas das forças máximas de tração no contraventamento

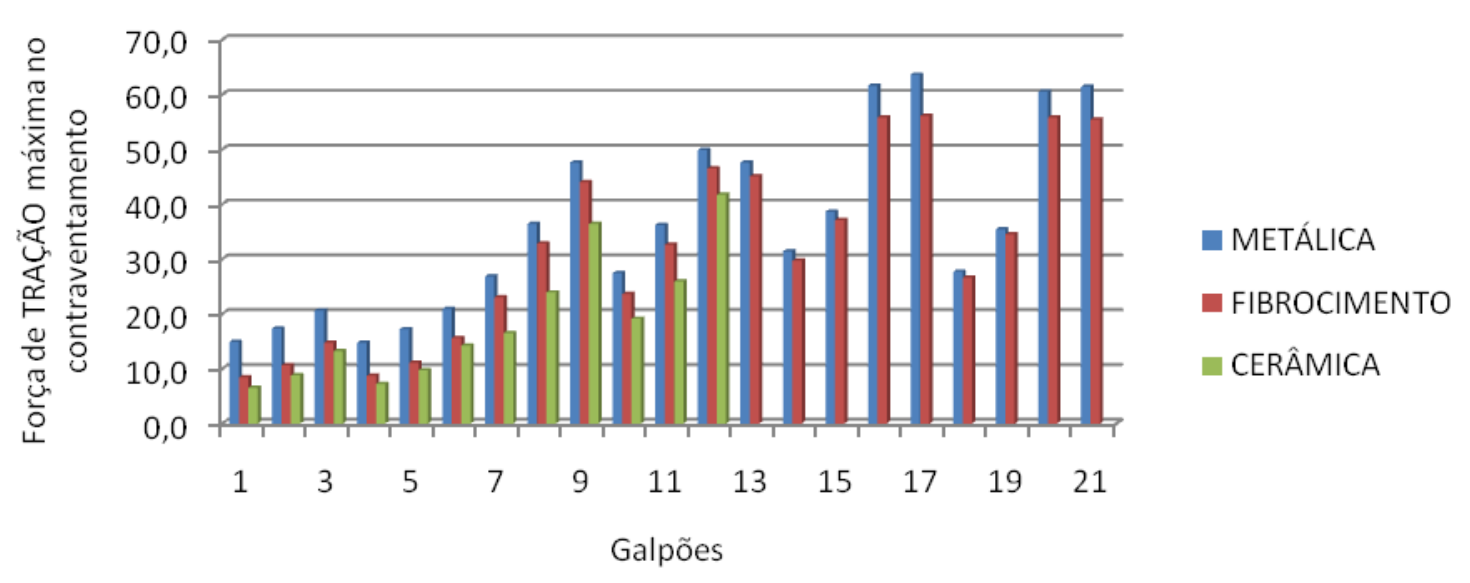




\subsection{Considerações sobre a análise dos resultados}

O método da NBR 7190 (1997) apresentou todos os valores inferiores aos modelos computacionais, com exceção do Galpão 18 (24x96 z=3 e=3 n=31).

O método do EUROCODE 5 mostrou valores superiores aos observados em cerca 75\% dos modelos. Para os modelos com contraventamento de madeira a margem de valores superiores aos dos modelos computacionais sobe para $85 \%$.

O método apresentado por UNDERWOOD (2000) apresentou valores superiores em cerca $90 \%$ dos modelos estudados.

O método do SABS 0163 apresentou valores inferiores a quase todos os modelos.

As mudanças de tipo de treliça (Howe e Pratt), de classe de resistêcia (C30 e C50) e de tipo de telha (metálica, fibrocimento e cerâmica) nos modelos, apresentaram valores próximos, sem variações significativas, das forças de compressão máximas nos banzos das treliças e no contraventamento.

O modelo com contraventamento em madeira apresentou valores das forças máximas no contraventamento inferiores aos modelos com contraventamento de aço. 


\section{CONCLUSÕES}

O método da NBR 7190 (1997) de estimativa da força mínima em barras integrantes do contraventamento de estruturas treliçadas leva a valores inferiores aos obtidos nos modelos tridimensionais que consideram o funcionamento global da estrutura. Apresentam, assim, valores que possam comprometer a segurança das referidas estruturas.

Os métodos da NBR 7190 (1997) e do SABS 0163 apresentaram quase todos os valores inferiores aos obtidos na análise tridimensional.

As formulações propostas pelo EUROCODE 5 apresentaram valores das forças no contraventamento próximos aos obtidos nos modelos computacionais, com 75\% de valores superiores.

O método apresentado por UNDERWOOD (2000) apresentou 90\% dos valores superiores aos obtidos nos modelos tridimensionais.

O aumento do número de treliças paralelas nos modelos tridimensionais não provocou aumento da força no contraventamento, ao contrário de que ocorre nos métodos baseados na instabilidade lateral dos elementos.

A variação do tipo de treliça entre Howe e Pratt, da classe de resistência da madeira entre C30 e C50, e do tipo de telha entre metálica, fibrocimento e cerâmica, apresentou valores de forças no contraventamento similares, mostrando-se praticamente neutra na análise realizada. Também não foram observadas diferenças significativas da força no contraventamento quando variados os espaçamentos entre treliças de três para quatro metros. De acordo com o que consta no Gráfico 37, Gráfico 39 e Gráfico 40.

Portanto, os valores das forças máximas nos elementos do contraventamento, segundo o funcionamento global das estruturas estudadas neste trabalho, se relacionam com o vão da treliça e a altura do pilar que a sustenta, os quais determinam as ações dos ventos na estrutura.

Sugere-se, finalmente, que os projetos de estruturas treliçadas para cobertura sejam desenvolvidos considerando o funcionamento global da estrutura ou outras análises mais 
refinadas, tendo em vista as ferramentas computacionais hoje disponíveis à análise estrutural. Deste modo, serão evitados eventuais problemas decorrentes da adoção dos parâmetros propostos pela NBR7190 (1997) no tocante às forças nas barras integrantes do contraventamento. 


\section{REFERÊNCIAS BIBLIOGRÁFICAS}

ABNT - ASSOCIAÇÃO BRASILEIRA DE NORMAS TÉCNICAS. NBR 6120 Cargas para o cálculo de estruturas de edificações. Rio de Janeiro: ABNT, 1980.

ABNT - ASSOCIAÇÃO BRASILEIRA DE NORMAS TÉCNICAS. NBR 6123 Forças devidas ao vento em edificações. Rio de Janeiro: ABNT, 1988.

ABNT - ASSOCIAÇÃO BRASILEIRA DE NORMAS TÉCNICAS. NBR 7190 Projeto de estruturas de madeira. Rio de Janeiro: ABNT, 1997.

BARALDI, L. T. Ação diafragma de cobertura aplicada ás construções em pórtico de madeira. São Carlos: SET/EESC/USP (tese de doutorado), 2001.

CALIL JR., C. Treliças de madeira para coberturas. Apostila. São Carlos : EESC/SAP, s.d.

CALIL JR., C.; ROCCO LAHR, F. A.; DIAS, A. A. Dimensionamento de Elementos Estruturais de Madeira. Barueri: Manole, 2003.

CALIL JR., C. South America Timber Structures Code. Concepción, Chile: Proceedings of the $51^{\text {st }}$ International Convention of Society of Wood Science and Technology, 2008.

CORDOVIL, F. A. B. Contraventamento de peças comprimidas. São Carlos: vii ebramem, 2000.

FRUCHTENGARTEN, J. Dimensionamento de estruturas de aço. São Paulo: Núcleo de Tecnologia da Construção Metálica, 1988.

GONÇALVES, R. M. et al. Ação do vento nas edificações: teoria e exemplos. São Carlos: SET/EESC/USP, 2007.

MADSEN, B. Structural Behaviour of Timber. Vancouver: Timber Engineering, 1992.

MADSEN, B. Behaviour of Timber Connections. Vancouver: Timber Engineering, 2000. 
PFEIL, W.; PFIEL, M. Estruturas de Madeira. Rio de Janeiro: LCT, 2003.

RAMPAZZO, S. E.; SPONCHIADO, M. O uso da madeira de reflorestamento na construção civil em enfoque na habitação. Erechim: Revista de Pesquisa e Pós-Graduação, 2000.

ROCCO LAHR, F. A. et al. Contribuição do contraventamento no cálculo de estruturas treliçadas espaciais de madeira. Londrina: xi ebramem, 2008.

SANTOS, L. B.; MORALES, E. A. M.; ROCCO LAHR, F. A. Algumas considerações sobre os critérios propostos pela NBR 7190(1997) para a verificação da segurança em pilares de madeira. Uberlândia: viii ebramem, 2002.

SANTOS, L. B.; ROCCO LAHR, F. A.; CÉSAR, S. F. Curvas de flambagem para dimensionamento de elementos comprimidos em estruturas de madeira. Cuiabá: ix ebramem, 2004.

UNDERWOOD, C. R. Permanent bracing design for MPC Wood roof truss webs and chords. Blacksburg: Virginia Polytechnic Institute and State University (master of science thesis), 2000. 